\title{
Air-Sea Interaction in the Tropical Atlantic
}




\section{Thesis committee}

\section{Promotor:}

Prof. Dr W. Hazeleger

Special Professor, Climate Dynamics

Wageningen University \& Research

\section{Co-promotor:}

\section{Dr R.J. Haarsma}

Senior Scientist, Research \& Development of Weather and Climate Models (RDWK) Royal Netherlands Meteorological Institute (KNMI), De Bilt

\section{Other members:}

Prof. Dr A.J.F. Hoitink, Wageningen University \& Research

Prof. Dr J. Lübbecke, GEOMAR Helmholtz Centre for Ocean Research Kiel, Germany Prof. Dr B. Rodriguez-Fonseca, University Complutense of Madrid, Spain Prof. Dr R.T. Sutton, University of Reading, UK

This research was conducted under the auspices of Graduate School for Socio-Economic and Natural Sciences of the Environment (SENSE) 


\section{Air-Sea Interaction in the Tropical Atlantic}

Anna-Lena Deppenmeier

\section{Thesis}

submitted in fulfilment of the requirements for the degree of doctor at Wageningen University by the authority of the Rector Magnificus

Prof. Dr A.P.J. Mol,

in the presence of the

Thesis Committee appointed by the Academic Board to be defended in public

on November 82019

at 1:30 p.m. in the Aula. 
Anna-Lena Deppenmeier

Air-Sea Interaction in the Tropical Atlantic

158 pages.

PhD thesis, Wageningen University, Wageningen, the Netherlands (2019)

With references, with summary in English

DOI: https://doi.org/10.18174/496766

ISBN: 978-94-6395-049-7 


\section{Summary}

Sea surface temperatures in the tropical Atlantic ocean influence weather and climate patterns on the bordering continents and beyond. In particular, the West African Monsoon and rainfall and drought over South America are tightly linked to tropical Atlantic sea surface temperatures (SST). Accurate simulation of oceanic processes is important because it could enable prediction of societally relevant events, such as drought seasons or the onset of the monsoon. However, state-of-the-art Coupled General Circulation Models (CGCMs), which could be used for such predictions, are known to be limited by long-standing biases in the tropical Atlantic. For example, they typically overestimate sea surface temperatures in the east of the basin on both the equator and to the south. Furthermore, the thermocline in the ocean is unrealistically deep in the east and shallow in the west to an extent that reverses the slope of the observed thermocline. These oceanic biases are associated to considerable atmospheric biases, including overestimation of precipitation south of the inter-tropical convergence zone (ITCZ) and underestimation of easterly winds.

In this thesis we investigate the origin of the warm SST bias using several approaches. We (i) make use of the coupled model inter-comparison project phase 5 (CMIP5) database, (ii) perform sensitivity experiments using a coupled ocean-atmosphere single column model (SCM), (iii) a state-of-the-art CGCM, and (iv) investigate feedback processes from in-situ buoy observations and reanalysis data.

In the tropics, where sea surface temperatures are high, air-sea coupling is strong. The ocean and the atmosphere influence each other, which can give rise to well-organised oscillatory patterns of recurring sea surface temperature variability. One such pattern is the Atlantic Niño. In chapter 2 we investigate whether the Atlantic Niño can be attributed to the Bjerknes Feedback, an air-sea coupled processes connecting eastern-equatorial SST, western-equatorial zonal winds, and eastern equatorial upper ocean heat content. The Atlantic Niño is characterised by anomalous formation of the eastern-equatorial "cold tongue". The cold tongue is part of the SST seasonal cycle in the eastern tropical Atlantic, emerging each summer between the months of May and August. Inter-annual variability on the magnitude of the associated cooling leads to this SST mode of variability with a periodicity of 2-5 years. In chapter 2 , we demonstrate that the feedback is active by cross- 
correlating SST, zonal wind stress, and ocean heat content obtained from ERA-Interim and ORA-S4 reanalysis data. We perform the same correlation analysis on model output from 36 models of the CMIP5 ensemble. The CGCMs reproduce the impacts of SST on zonal winds, as well as the effects of zonal winds on heat content, albeit with a one-month lag compared to observations. However, the local east-equatorial coupling between heat content and SST is not well reproduced by the models. Subsurface temperature analysis indicates that incorrect positioning and insufficient temperature variability around the thermocline are responsible for this behaviour. The CGCMs are able to reproduce some surface-subsurface coupling later in the year, when the influence of wind stress on heat content has subsided. The Bjerknes Feedback loop is therefore not closed in state-of-theart CGCMs.

The lacking subsurface-surface coupling in boreal summer prevents the accurate simulation of the observed summer-time cooling. In chapter 3, we investigate the strong cooling with a novel coupled ocean-atmosphere single column model (SCM) version of the stateof-the-art GCM EC-Earth. We place the SCM at a PIRATA mooring site, which enables us to compare the simulation to high temporal-resolution in-situ observations. The SCM captures the SST evolution well for the first five days of the simulation, after which it develops a SST bias strongly resembling that of its three dimensional counterpart. This bias can be reduced substantially by enhancing the turbulent mixing efficiency in the ocean vertical mixing parameterisation within the range constrained by observations. Sensitivity experiments in which we eliminate the atmospheric temperature and moisture biases also reduce the SST bias, but the effects are less pronounced than when enhancing the ocean vertical mixing efficiency. Forcing the SCM with observed surface shortwave radiation or wind, however, does not improve the simulation. The warm SST bias extends down to the upper few meters of the ocean, artificially stabilising the simulated ocean column. We replaced the vertical profiles of temperature and salinity at the point where the turbulent coefficients are calculated in the ocean model, reducing the SST bias by $30 \%$. Our findings highlight the importance of the representation of upper ocean vertical mixing for accurate simulation of the rapid summer-time cooling in the south-eastern tropical Atlantic.

We have demonstrated the impact of enhanced upper ocean vertical mixing with the coupled atmosphere-ocean single column model. In chapter 4, we construct an upper ocean heat budget to determine whether the bias in the three-dimensional CGCM ECEarth also arises from insufficient representation of vertical mixing. We perform the budget analysis on fully initialised seasonal hindcasts, which start from observational estimates and then evolve freely. This approach enables us to trace the development of the rapidly establishing bias rather than analysing the equilibrated bias. Our heat budget analysis reveals that subgrid-scale processes are indeed the prevailing positive contributors to the build-up of the warm SST bias, both in the south-eastern tropical Atlantic and in an area below the summer inter-tropical convergence zone (ITCZ). We test the enhanced 
mixing parameterisation applied in the SCM simulation in the three-dimensional global coupled climate model in climate experiments with historical forcing. To this end, one experiment is performed with the default parameterisation and another with the enhanced mixing. The present-day SST bias is reduced under enhanced mixing, in line with results from the SCM. Further, the ITCZ beneficially strengthens and moves to the north, in association with the reduction of the SST bias. By continuing the experiments until the end of the 21st century under the representative concentration pathway scenario 8.5, we investigate the sensitivity of the climate change signal to enhanced ocean mixing. Future temperatures are projected to remain $0.5^{0} \mathrm{C}$ colder in boreal winter and spring, while summer and fall temperatures are projected warmer by the same magnitude. The ITCZ is projected to be stronger and narrower in the future, more so in the sensitivity experiment than in the control. Overall, the findings of the first three technical chapters suggest that mixing processes are important for summer-time cooling in the tropical Atlantic, but that they are not adequately captured in present default parameterisation schemes in climate models.

In chapter 5 we further investigate vertical mixing in the south-eastern tropical Atlantic and below the summer ITCZ from in-situ observations and reanalysis data. Heat budget analysis on reanalysis data reveals that vertical mixing largely contributs to the SST cooling in both regions. Lead-lag cross-correlation analysis further demonstrates that feedbacks between upper ocean stratification, ocean vertical mixing, SST, and shortwave radiation shape the climate variability in both regions. The identified feedback acts on short timescales of days to weeks, and is positive in the south-eastern tropical Atlantic and negative below the ITCZ due to differences in the typical cloud types for the two regions. For example, the ITCZ domain is dominated by deep convective clouds that form above warm SST anomalies. The resulting increase in cloud-cover reduces surface shortwave radiation, and ultimately decreases upper ocean stratification, and enhances vertical mixing. Vertical mixing cools the surface layer by entraining cold water from below and as a result reduces the initial SST anomaly. In contrast, in the south-eastern tropical Atlantic, a warm SST anomaly decreases overlying stratocumulus cloud-cover by de-stabilising the atmospheric column. This leads to increased surface shortwave radiation and ocean stratification, suppressing upper ocean vertical mixing. In this region, the original warm SST anomaly is reinforced. Correct model-representation of this feedback and its geographical imprint could improve the representation of ocean vertical mixing, and therefore improve climate model simulations.

The findings in this thesis highlight the important role of upper ocean vertical mixing to climate variability in the tropical Atlantic. Inaccurate representation of this ocean process leads to warm SST biases. These SST biases in turn can cause atmospheric biases, which perpetuate oceanic biases. Ehancing ocean vertical mixing in the parameterisation scheme reduces the warm SST bias, and atmospheric biases associated to it. 



\section{Contents}

Page

Summary $\quad$ v

Contents $\quad$ ix

Chapter 1 Introduction $\quad 1$

1.1 Tropical Atlantic Climate . . . . . . . . . . . . . . . . . 1

1.2 The seasonal cycle . . . . . . . . . . . . . . . . . . 3

1.2.1 Sea surface temperature . . . . . . . . . . . . . . 3

1.2.2 Zonal winds . . . . . . . . . . . . . . . . . . 4

1.2.3 Thermocline ........................ 4

1.2.4 Intertropical Convergence Zone . . . . . . . . . . . . . . 6

1.3 Interannual variability $\ldots \ldots \ldots \ldots \ldots$

1.4 Data . . . . . . . . . . . . . . . . . . . . 10

1.5 Climate Models . . . . . . . . . . . . . . . . . . . 10

1.5.1 Coupled Clobal Climate Models . . . . . . . . . . . . . . . . . 10

1.5.2 Single Column Models . . . . . . . . . . . . . . . . . . 11

1.5.3 The CMIP5 Database . . . . . . . . . . . . . . . . 12

1.6 In situ observations . . . . . . . . . . . . . . . . . . . . . 13

1.7 Reanalysis data . . . . . . . . . . . . . . . . . . . . . . . . . . . . . . . . . . . . .

1.8 Aim and Outline . . . . . . . . . . . . . . . . 14

Chapter 2 The Bjerknes Feedback in the Tropical Atlantic 17

2.1 Introduction . . . . . . . . . . . . . . . . . . . 19

2.2 Data and Methodology . . . . . . . . . . . . . . . . 20

2.3 Bjerknes Feedback in Reanalysis Data . . . . . . . . . . . . . . . 21

2.4 Comparison between Reanalysis and Model Output . . . . . . . . . . 28

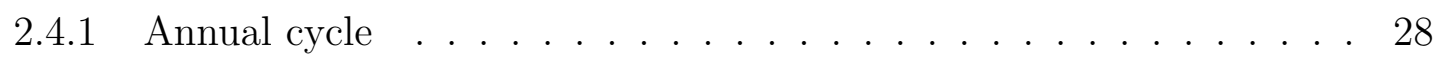

2.4.2 Pattern Correlation Analysis . . . . . . . . . . . . . . . . 30

2.4 .3 Regression Values . . . . . . . . . . . . . . . . . . . 35

2.4.4 Subsurface Structure . . . . . . . . . . . . . . . . . . . . . . . . . . . . . . . . . . . . . . .

2.5 Conclusion . . . . . . . . . . . . . . . . . . . . 40 
Chapter 3 The South-Eastern Tropical Atlantic SST bias investigated with a coupled atmosphere-ocean single column model at a PIRATA mooring site.

3.1 Introduction . . . . . . . . . . . . . . . . . . . . 45

3.2 Model description . . . . . . . . . . . . . . . . . . . . . 47

3.2.1 Turbulent Vertical Mixing in the Ocean . . . . . . . . . . . . . . 50

3.3 Experimental Setup . . . . . . . . . . . . . . . . . . . . . . . . . . . . . . 52

3.3.1 Sensitivity Experiments . . . . . . . . . . . . . . 52

3.4 Results . . . . . . . . . . . . . . . . . . . . 53

3.4.1 Temperature bias in the Single Column Model . . . . . . . . . . . 53

3.4 .2 Surface forcing . . . . . . . . . . . . . . . . 56

3.4.3 Ocean model . . . . . . . . . . . . . . . . 61

3.5 Discussion and Summary . . . . . . . . . . . . . . . . . . . . . . . 69

Chapter 4 The effect of vertical ocean mixing efficiency on the tropical $\begin{array}{ll}\text { Atlantic in a coupled global climate model. } & 71\end{array}$

4.1 Introduction . . . . . . . . . . . . . . . . . . . . . 73

4.2 Data and Methodology . . . . . . . . . . . . . . . 74

4.3 Results . . . . . . . . . . . . . . . . . 76

4.3.1 Biases in the historical simulation and in the seasonal hindcasts . . 76

4.3.2 Upper ocean heat budget . . . . . . . . . . . . . . . . 80

4.3.3 Sensitivity experiments . . . . . . . . . . . . . . . . . 82

4.4 Summary and Discussion . . . . . . . . . . . . . . . . . . . . 97

Chapter 5 Coupled ocean mixing and cloud feedbacks in the tropical At$\begin{array}{ll}\text { lantic } & 101\end{array}$

5.1 Introduction . . . . . . . . . . . . . . . . . . 103

5.2 Data and methods . . . . . . . . . . . . . . . . 104

5.3 In situ data analysis . . . . . . . . . . . . . . . . . . . . . . . 105

5.4 Reanalysis data analysis . . . . . . . . . . . . . . . . 107

5.5 Discussion . . . . . . . . . . . . . . . . . . . . 110

Chapter 6 Synthesis and Recommendations 113

6.1 Overview of the present research . . . . . . . . . . . . . . . . 114

6.2 Recommendations . . . . . . . . . . . . . . . . . . . . . 123

6.2.1 Single Column Model Studies . . . . . . . . . . . . . . . . . 123

6.2.2 Coupled Global Climate Model Studies . . . . . . . . . . . . . . . . 125

6.2.3 Analysis from Observations . . . . . . . . . . . . . . . 126

6.3 Closing remarks . . . . . . . . . . . . . . . . 126

$\begin{array}{lr}\text { References } & 127\end{array}$ 
$\begin{array}{lr}\text { Acknowledgements } & 139\end{array}$

Peer-reviewed publications 



\section{Chapter 1}

\section{Introduction}

\subsection{Tropical Atlantic Climate}

Tropical Atlantic climate impacts the lives of people on the bordering continents and beyond. The ocean basin is narrow and tightly flanked by South America and Africa, two large land masses. Local people depend on the ocean to provide them with food. At its eastern boundary, cold and nutrient-rich water from the deeper ocean reaches the surface. This offers ideal conditions for fish to flourish, and for the fishing industry. Agriculture in the semi-arid regions of Africa and South America is dependent on rainfall, which is tightly linked to tropical Atlantic sea surface temperatures. The ocean's temperature anomalies influence rainfall and droughts in South America (Moura and Shukla, 1981; Lamb et al., 1986; Zhou and Lau, 2001; Giannini et al., 2004; Yoon and Zeng, 2010) and the West African monsoon (Rouault et al., 2003; Okumura and Xie, 2004; Kucharski et al., 2009). In the Brazilian north-eastern state Nordeste alone, 50 million people depend on rainfall influenced by Atlantic sea surface temperature anomalies (Nobre and Shukla, 1996; Uvo et al., 1998). The West African summer monsoon, on the other side of the ocean, supplies the continent with direly needed water. Drought and rain seasons in West Africa are closely linked to tropical Atlantic sea surface temperature anomalies (Giannini et al., 2003; Shanahan et al., 2009; Rodríguez-Fonseca et al., 2011; Polo et al., 2008). The influence of tropical Atlantic sea surface temperatures (SSTs) extends beyond the bordering continents. SST anomalies assert influence on the North Atlantic Oscillation (Okumura et al., 2001; Haarsma and Hazeleger, 2007; García-Serrano et al., 2008), rainfall in North America (Kushnir et al., 2010) and heatwaves in Europe (Cassou et al., 2005; Sutton and Hodson, 2005). Furthermore, teleconnections influencing the El Niño Southern Oscillation have been suggested (Ham et al., 2013; Martín-Rey et al., 2015). The tropical Atlantic is a key element in the climate system. If we wish to understand, simulate, and predict societal relevant problems, we first need to understand climate variability of the ocean. 
Unfortunately, coupled global climate models (GCMs) suffer from large biases in the region (Davey et al., 2002; Stockdale et al., 2006; Wang et al., 2014). Warm ocean surface temperature biases reach values as high as $6^{\circ} \mathrm{C}$ (Toniazzo and Woolnough, 2014). Even though the new generation of climate models have improved, for example in their representation of the Pacific (Bellenger et al., 2014), the large biases in the tropical Atlantic remain (Richter and Xie, 2008; Richter et al., 2014b). As a consequence, the tropical Atlantic has received substantial attention, as efforts are made to understand the model deficiencies. Among the proposed origins of the sea surface temperature bias are atmospheric biases, such as a westerly wind bias (Richter et al., 2012; Voldoire et al., 2014; Koseki et al., 2018; Voldoire et al., 2019; Goubanova et al., 2019), atmospheric moisture bias (Hourdin et al., 2015), and excessive solar radiation through a deficiency of low level clouds (Huang et al., 2007; Hu et al., 2008, 2011; Zuidema et al., 2016). But even with simulations at very high spatial ocean resolution, forced by observed wind stress, the sea surface temperature bias cannot be eliminated (Small et al., 2014; Xu et al., 2014b). The ocean model contributes to the bias development (Song et al., 2015) and sustains it (Toniazzo and Woolnough, 2014).

In the tropics, the ocean and the atmosphere are tightly coupled. Shallow mixed layers can relatively easily be warmed by the abundant incoming radiation, and moist air rises readily above warm SSTs, impacting the atmospheric circulation. Tropical Atlantic sea surface temperatures show well-organised recurring patterns. These patterns are also called climate modes, or modes of variability. Air-sea coupled modes in which atmospheric variables, such as wind and moisture, co-vary with oceanic variables, such as sea surface temperature and upper ocean heat content, explain a considerable fraction of the sea surface temperature variability. Two coupled modes causing inter-annual variability have been identified early in the literature: the Atlantic Niño (Merle, 1980; Carton and Huang, 1994), named due to its resemblance to the El Niño Southern Oscillation in the Pacific (Zebiak, 1993), and an inter-hemisphere dipole mode (Moura and Shukla, 1981; Servain, 1991). The latter acts primarily on decadal timescales (Servain, 1991; Chang et al., 1997), and is strong in boreal spring.

In this thesis, we focus on variability on seasonal and inter-annual timescales, linked to the Atlantic Niño. The Atlantic Niño dominates in boreal summer, when sea surface temperatures in the eastern tropical Atlantic undergo rapid cooling, and the West African Monsoon rain season begins.

The Atlantic Niño has been studied and reviewed, for example in (Ruiz-Barradas et al., 2000; Xie and Carton, 2004; Lübbecke et al., 2018). Still, to this date, there is lively discussion about its coupled nature. While it is generally accepted that ocean dynamics significantly contributes to its origin (Carton and Huang, 1994; Keenlyside and Latif, 2007; Wang and Chang, 2008; Ding et al., 2010; Brandt et al., 2011), alternative mechanisms for eastern equatorial sea surface temperature variability have been suggested only a few 
years ago (Richter et al., 2013; Nnamchi et al., 2015). If we assume that the Atlantic Niño arises from air-sea coupling which involves ocean dynamics, the mechanism driving both annual and inter-annual variability are very similar (Burls et al., 2011, 2012; Dippe et al., 2018a). Indeed, inter-annual variability peaks simultaneously with the eastern equatorial Atlantic SST minimum, which demonstrates the phase-locking of the seasonal cycle and inter-annual variations (Lübbecke et al., 2018).

In the following sections, we consider three important variables for studying air-sea interaction: sea surface temperature, zonal wind stress, and the thermocline, the steepest vertical temperature gradient in the ocean column. Sea surface temperature couples the atmosphere to the ocean by influencing atmospheric stability. Wind stress affects the ocean surface temperature and currents. The thermocline offers information about the upper ocean heat content. We will first look at the seasonal cycle in reanalysis in Section 1.2 before moving on to inter-annual variability in Section 1.3.

\subsection{The seasonal cycle}

\subsubsection{Sea surface temperature}

Sea surface temperature in the tropical Atlantic varies greatly during the year. In late boreal winter and spring (January - April) zonal temperature gradients are small (Fig. 1.1). Especially along the equator, temperatures are uniformly higher than $27^{\circ} \mathrm{C}$ (Fig. 1.2a). The warm band extends approximately five degrees to the north and ten to the south. Within this region, the meridional SST gradient is also weak. To the north and to the south of it, temperatures drop rapidly.

In May, at the end of boreal spring, the eastern tropical Atlantic cools strongly and rapidly (Fig. 1.1), and the equatorial zonal temperature gradients reverses (Fig. 1.2a). During the summer months, the sea surface on the eastern tropical equator cools by more than $4^{\circ} \mathrm{C}$ (Fig. 1.2b). Minimum temperatures are reached in August, when, simultaneously, the so called Atlantic cold tongue on the equator reaches its maximum (Figure 1.2c). The cold water in the east of the equatorial Atlantic seemingly joins the northward extending cold region from the very south east, where temperatures have dropped below $19^{\circ} \mathrm{C}$.

Starting in September, the cold tongue recedes, sea surface temperatures rise, and the zonally warm band returns. In contrast to the rapid cooling, the warming happens relatively slowly. This asymmetry is noteworthy: it only takes four months to drastically cool the south-eastern and equatorial Atlantic, but it takes eight months before temperatures reach their maximum again in April. 
Figure 1.1: Seasonal cycle of surface temperatures in the tropical Atlantic obtained from ERA-Interim reanalysis data (Dee et al., 2011) (1979-2013), summer months highlighted with frames.

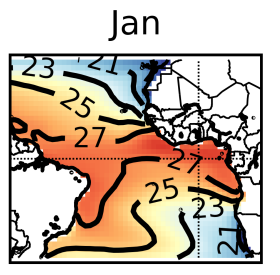

May
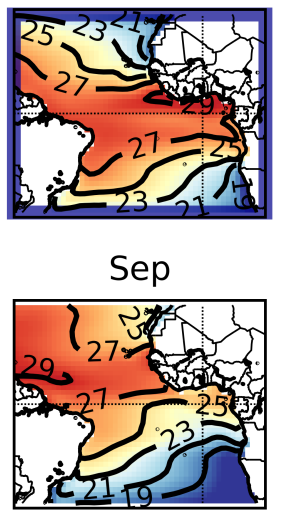

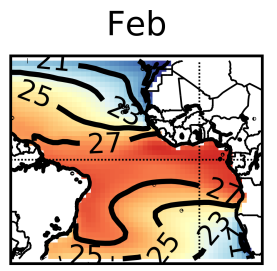

Jun

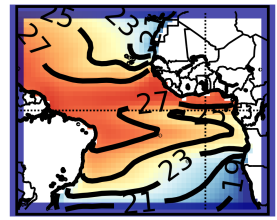

Oct

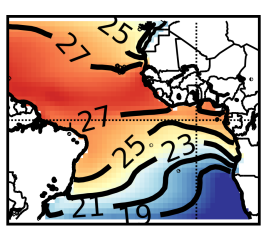

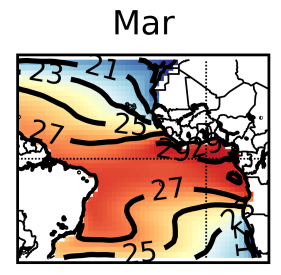

Jul

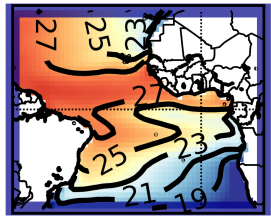

Nov

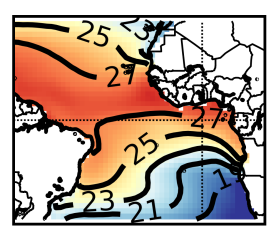

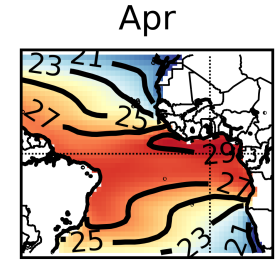

Aug

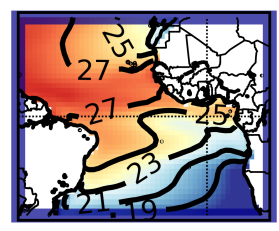

Dec

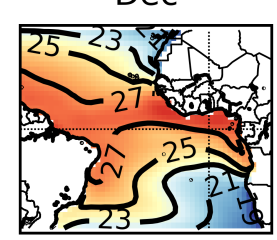

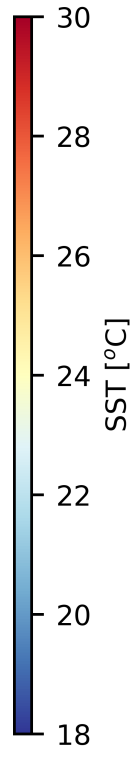

\subsubsection{Zonal winds}

Zonal winds along the equator are easterlies, they flow from east to west. In spring, the winds in the western equatorial Atlantic are at their weakest (Figure 1.3). This coincides with the peak of eastern Atlantic sea surface temperatures, and a minimized zonal temperature gradient. The trade winds relax along the zonally uniform sea surface temperatures. During summer, the western equatorial wind picks up pace rapidly. At that time, the sea surface begins to cool in the east, and the zonal temperature gradient increases. The gradient enhances the Walker circulation, which describes warm air rising in the west above high SSTs, and subsiding in the east, above low SSTs. This is accompanied by westward movement of air at the surface, and eastward flow aloft. Along equatorial winds are easterlies, so when the Walker circulation strengthens, along-equatorial winds increase (Figure 1.3). Zonal wind stress peaks in August (Figure 1.3b), when the eastern equatorial cold tongue is at its maximum and the temperature gradient is largest (Figure 1.2).

\subsubsection{Thermocline}

The seasonal cycle also affects the ocean below the surface. Zonal winds "push" water to the west of the basin along the equator, causing the thermocline (the steepest vertical temperature gradient in the ocean) to shoal in its wake. In the summer months, when 
Figure 1.2: Seasonal cycle of surface temperatures in the tropical Atlantic obtained from ERA-Interim reanalysis data (Dee et al., 2011) (1979-2013). Panel a) shows the sea surface temperature evolution throughout the year in the $3^{\circ} \mathrm{S}-3^{\circ} \mathrm{N}$ band along the equator. Panel $\mathrm{b}$ ) shows the average sea surface temperature in the $20^{\circ} \mathrm{W}-0^{\circ} \mathrm{E}, 3^{\circ} \mathrm{S}-3^{\circ} \mathrm{N}$ box, summer months are highlighted with shading, and panel c) shows the sea surface temperature in August, when the Atlantic cold tongue is at is maximum.

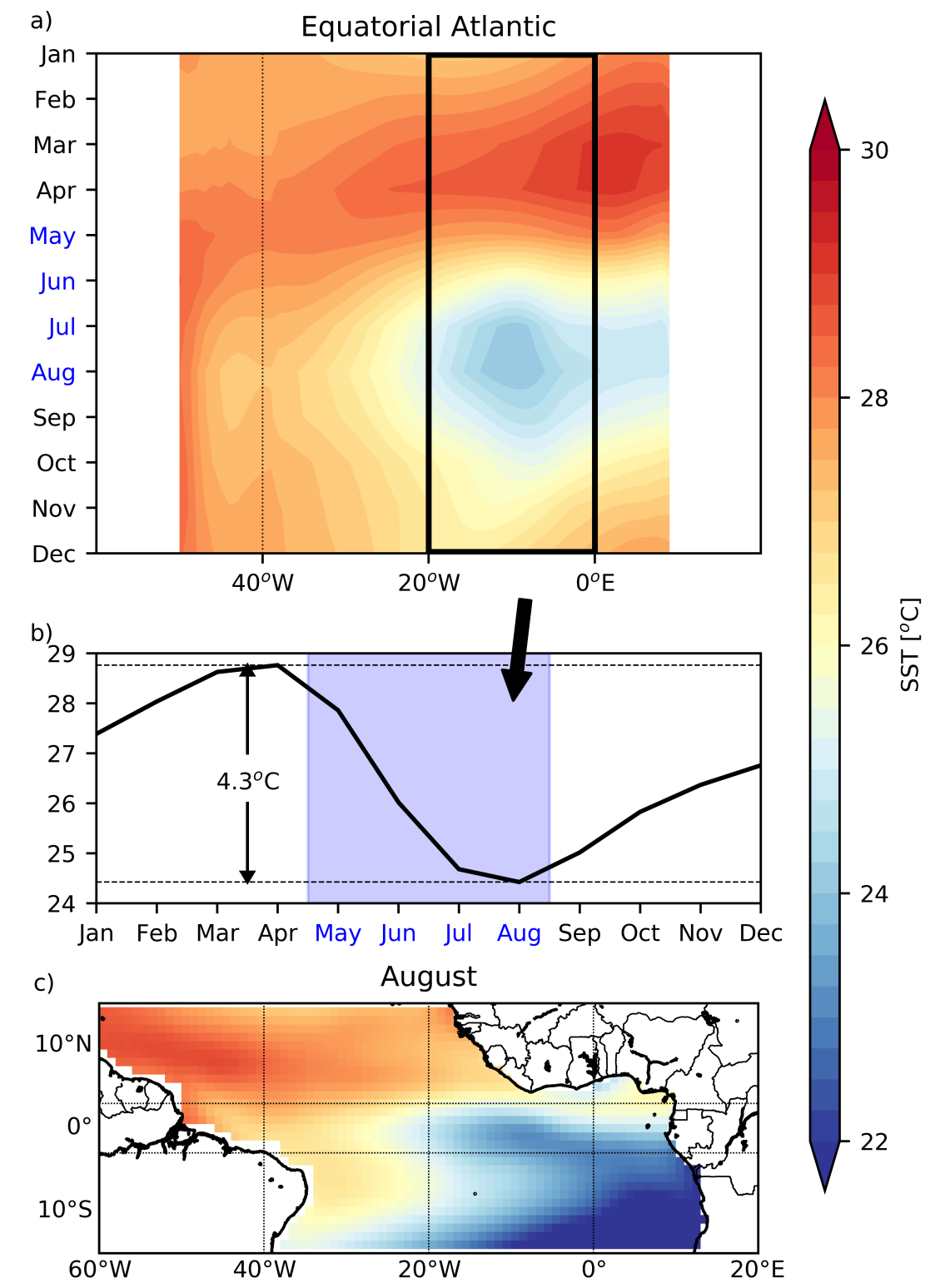

the winds increase, the eastern equatorial thermocline is at its shallowest (Figure 1.4). It deepens at the end of boreal summer, and reaches its maximum depth in fall. Throughout the year, the thermocline remains shallower in the east and deeper in the west. The extreme shallowness in summer makes the upper ocean layer in the eastern tropical Atlantic susceptible to influences from both the surface and the ocean below. At the surface, 
Figure 1.3: Seasonal cycle of eastward surface wind stress from ERA-Interim reanalysis data (Dee et al., 2011) (1979-2013). Panel a) shows the zonal wind stress development along the equatorial band, panel b) shows the seasonal cycle in the box between $40^{\circ} \mathrm{W}-20^{\circ} \mathrm{W}$ and $3^{\circ} \mathrm{S}-3^{\circ} \mathrm{N}$.

a)

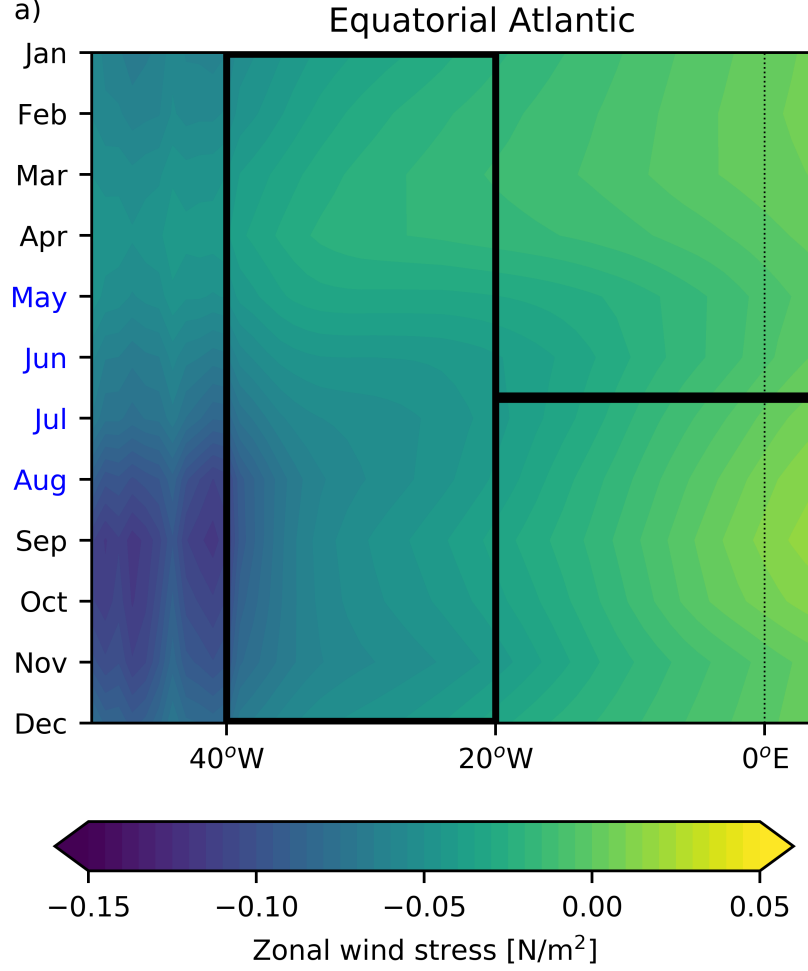

b)

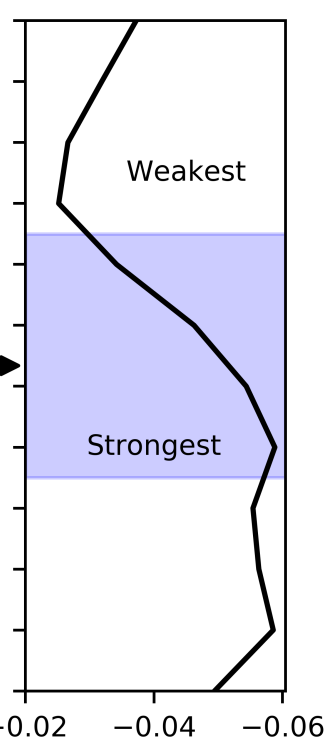

radiative and turbulent fluxes can assert large influences on the thin layer, which has a low heat capacity compared to a thicker mixed layer. From below, colder water can be entrained by vertical mixing processes. This asserts especially large influence on upper ocean temperatures if the well mixed layer is thin.

\subsubsection{Intertropical Convergence Zone}

The tropical Atlantic is spanned by a large scale rain band, the intertropical convergence zone (ITCZ, Figure 1.5). The maritime location of the ITCZ is closely connected to sea surface temperatures. It connects the two continental convection regions above South America and Africa. The rain band forms where trade winds from the south and the north converge and intensify the ascent of warm, moist air above high sea surface temperatures, giving rise to deep convection. Associated with this strong convection is a band of thunderstorms and monthly mean rainfall intensities of up to $15 \mathrm{~mm} /$ day (Biasutti et al., 2006). The ITCZ follows the zonal band of high sea surface temperatures, and therefore reaches its southernmost position in boreal spring, when it is located nearly on 
Figure 1.4: Thermocline depth evolution of the equatorial Atlantic a) throughout the year, and $b$ ) the annual mean. Obtained by determining the steepest vertical temperature gradient from ORAS4 ocean temperature data (Balmaseda et al., 2013).

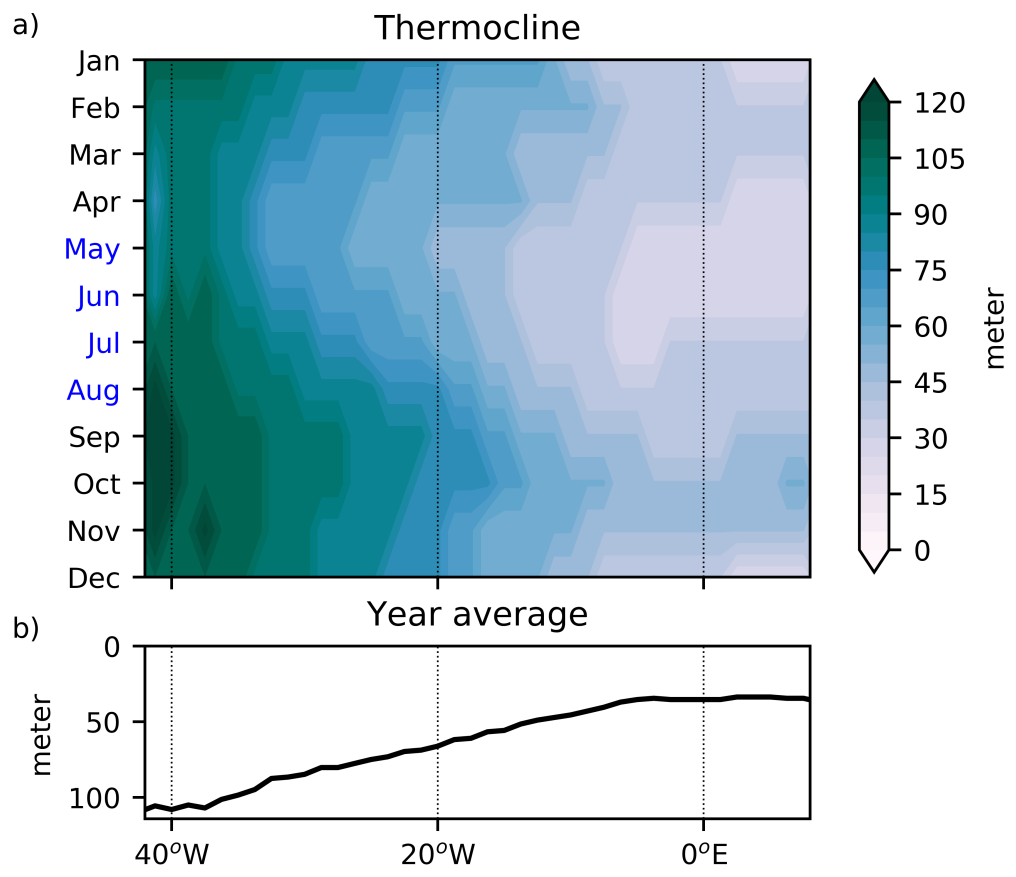

the equator. This is when zonal winds are at their weakest. The cooling connected to the equatorial cold tongue development in boreal summer moves the ITCZ north of the equator, and peak rainfall intensities move up to almost $10^{\circ} \mathrm{N}$ in September. The wind convergence also moves north, allowing the trade winds to increase the zonal wind stress along the equator.

The location of ITCZ depicts areas of deep convection, where clouds form as warm moist air rises deep into the troposphere. In other regions, where sea surface temperatures are colder, shallow convection or even descending motion occurs, giving rise to their own typical cloud types. Clouds, depending on their nature, react differently to an underlying sea surface temperature anomaly. For instance, deep convection will be triggered and increased above warm sea surface temperatures (Zhang, 1993; Tompkins, 2001; Giannini et al., 2003; Deckert and Dameris, 2008), while stratocumulus originate from descending motion, and will hence be reduced above a warm anomaly (Clement et al., 2009; Bellomo et al., 2015). The tropical Atlantic features both, and hence cloud responses can be very different from one location to another. 
Figure 1.5: The intertropical convergence zone throughout the year as marked by its precipitation intensity (obtained from ERA-Interim reanalysis data (Dee et al., 2011)). Note that while the annual march of the ITCZ is well captured in the ERA reanalysis, rainfall intensities over the tropical oceans are overestimated (Dee et al., 2011). Hence, this figure presents the spatial extent of the ITCZ, rather than its absolute rainfall values.

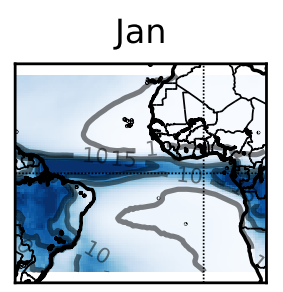

May

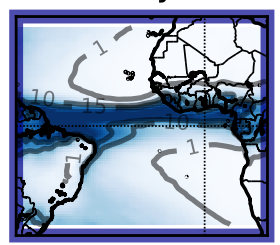

Sep

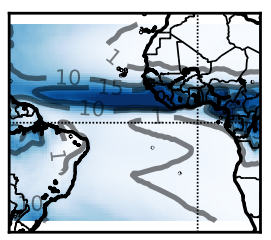

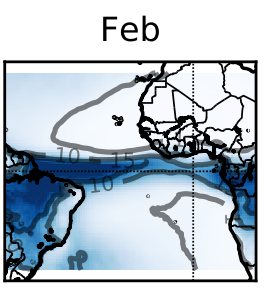

Jun

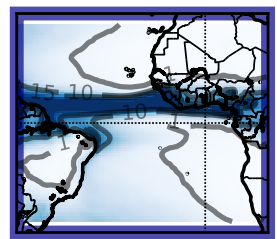

Oct

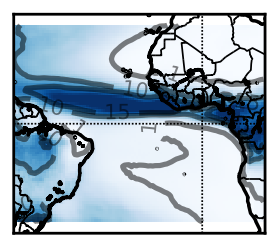

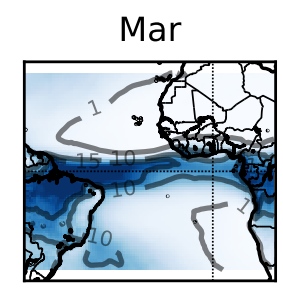

Jul

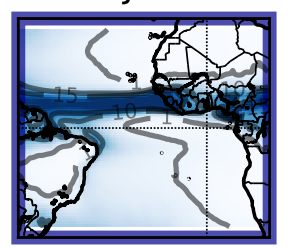

Nov

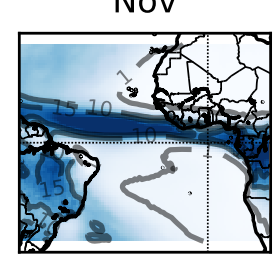

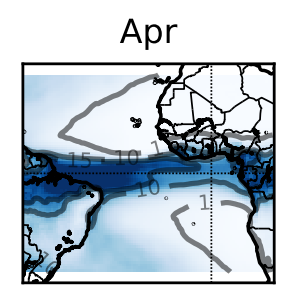

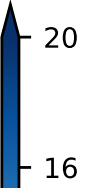

Aug

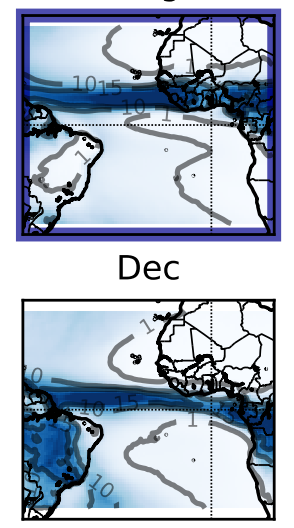

16

12

\subsection{Interannual variability}

Sea surface temperatures display variability on inter-annual timescales, closely phaselocked to the seasonal cycle. With a periocity of 2-5 years (Keenlyside and Latif, 2007; Brandt et al., 2011), the Atlantic Niño acts on short inter-annual timescales. It is characterised by anomalous warming of the cold tongue in boreal summer, and weakening of the zonal winds (Fig. 1.6).

If the sea surface temperature pattern is governed by a process with certain periodicity, it might offer predictability on the time scale of its cycle. We therefore try to understand where the variability arises from, and which observable variables are connected to it, so that we may predict its arrival in advance.

The Atlantic Niño explains a considerable fraction of sea surface temperature variance in boreal summer, even if the explained variance remains behind that of the analogous mode in the Pacific. Ruiz-Barradas et al. (2000), Sutton et al. (2000), and Keenlyside and Latif (2007) establish that this mode is due do the same air-sea coupled feedback mechanism as ENSO. The mechanism is called the Bjerknes Feedback (Bjerknes, 1969), and is schematically shown in Figure 1.7. It consists of three parts and makes a positive 
Figure 1.6: Regression of Tropical Atlantic sea surface temperature (colours), wind (arrows), and precipitation (contours) anomalies on the harmonic fit of sea surface temperature anomalies in ATL3 $\left(20^{\circ} \mathrm{W}-0^{\circ} \mathrm{E}, 3^{\circ} \mathrm{S}-3^{\circ} \mathrm{N}\right.$, black box) (a), and the corresponding time series of the harmonic fit of surface temperature anomlies (thick solid), fit to HadISST data (thin solid) and microwave optimally interpolated SST (thin dashed). Figure adapted from Brandt et al. (2011)
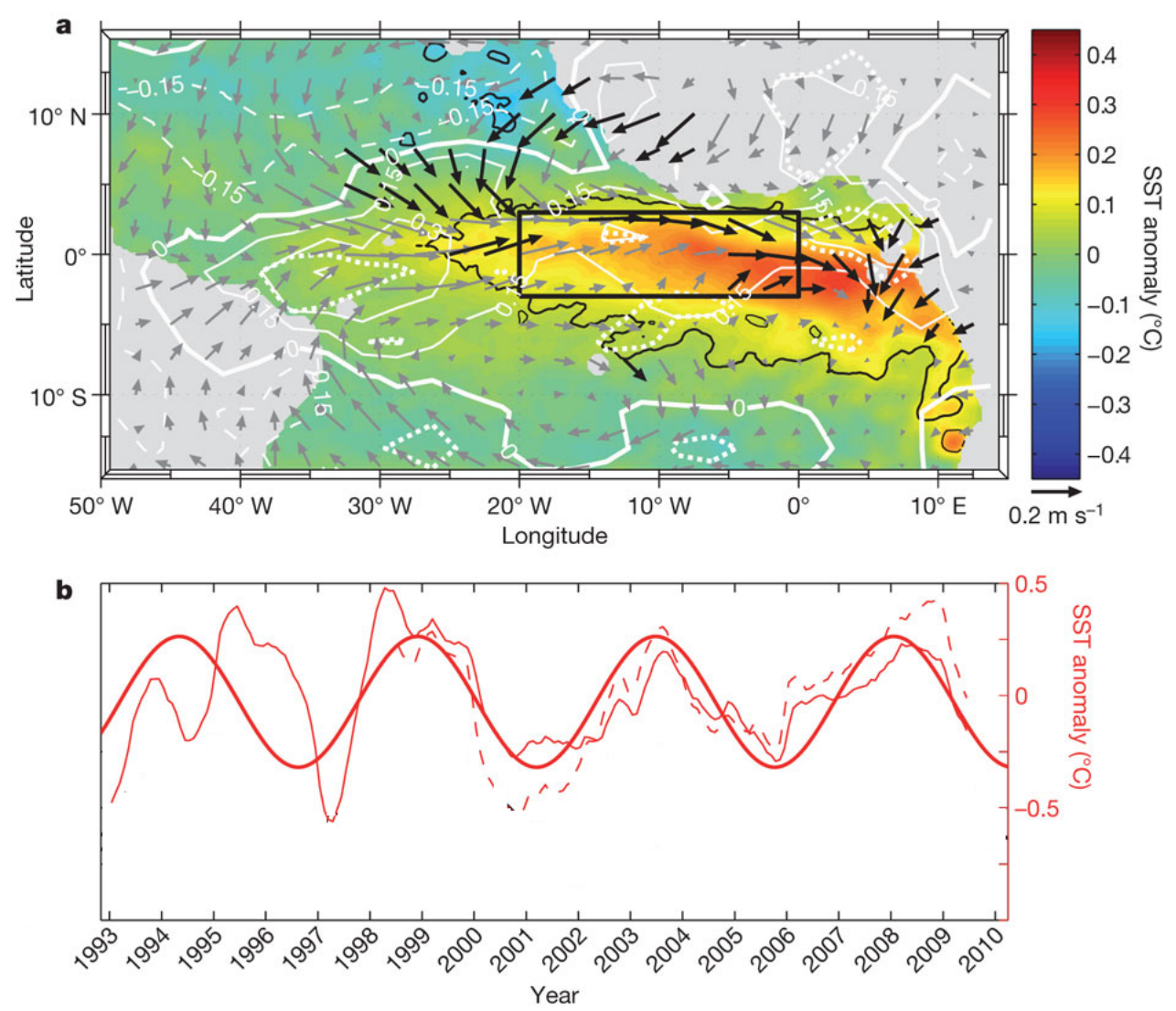

reinforcing feedback loop. Warm sea surface temperature anomalies in the east of the basin reinforce westerly wind anomalies in the west. A warm anomaly translates to a decreased zonal temperature gradient, which weakens the Walker circulation, and thereby the zonal surface wind. The wind stress anomalies in the west of the basin trigger equatorial Kelvin waves in the ocean. An equatorial Kelvin wave is a subsurface disturbance that travels along a well-defined wave guide, in this case, the equator. The wave propagates along the thermocline towards the east, where it deepens the mixed layer and leads to a build-up of heat content. Due to the deeper thermocline, cold water entrained into the mixed layer cools it less, locally reinforcing the warm sea surface temperature anomalies. 
Figure 1.7: Schematic of the Bjerknes Feedback mechanism in tropical oceans. The trade winds push warm surface water to the west and cause the thermocline in the east to shallow, allowing cool water from below it to assert large effects on the thin mixed layer temperature. In the atmosphere, moist air rises in the west above warmer water, giving rise to convective clouds. The air aloft travels eastward, where it descends. Figure adapted from Cravatte et al. (2016).

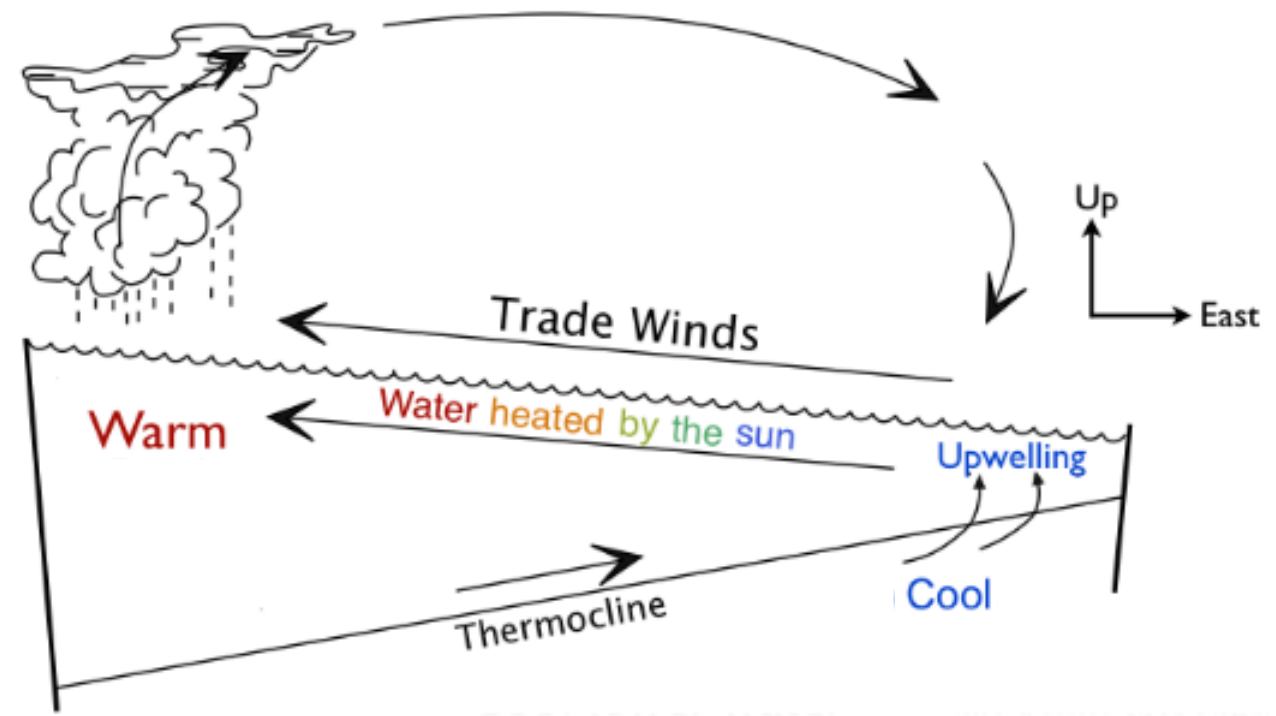

\subsection{Data}

To cast light on the processes that force SST variability in the tropical Atlantic, we have several tools at our disposal. Here, we briefly introduce the instruments we make use of in this thesis. They consist of numerical models that represent the physics of the natural world (Section 1.5.1), observations (Section 1.6), and models that incorporate observations to generate estimates of the natural world that cover large areas and time spans (Section 1.7). We introduce them in that order.

\subsection{Climate Models}

\subsubsection{Coupled Clobal Climate Models}

Coupled global climate models (GCMs) are invaluable tools for investigating the climate system. They enable researchers to focus on specific areas, but also evaluate the remote interactions with the area of interest. To efficiently model the large span of spatial and temporal scales of the climate system carefully considered compromises have to be made. We cannot expect to model, for example, cloud condensation at the same time as large 
scale processes, such as Rossby wave propagation. Each climate model is therefore based on a set of equations with which large scale dynamics are calculated, and several sets of equations which incorporate the effects of small scale processes without explicitly resolving them. Depending on the focus and intent of the model developer or user, these models are also called general circulation models, or Earth System models, when they incorporate the effects of human activity, for example via greenhouse gas forcing.

While the specifics vary between the different models, GCMs generally have the same structure. They consist of an atmosphere and an ocean component, which include representations for land surface processes and sea ice, respectively, and are coupled by a coupling software. The earth system is divided up into grid boxes, in which a set of differential equations describing the movement of fluids (derived from the Navier Stokes equations) is solved numerically. The grid boxes are stacked to form columns, and the columns are placed next to each other in a sphere to form the earth (Figure 1.8). Alternatively, discretisation in spectral space takes place. The fluid models solve their set of primitive equations, which consist of momentum equations and equations for temperature and moisture in case of the atmosphere, and temperature and salinity in case of the ocean. Each of these equations again can be decomposed into large scale dynamics, surface forcing, and parametrisation. The large scale dynamics are solved explicitly on the model grid and at the model time step. Surface forcing is received at coupling time steps at the interface between the different model components. Parametrisation takes into account small scale processes, which are not explicitly resolved in the model. Examples for the latter are cloud formation and radiative transfer in the atmosphere, and turbulent mixing in the ocean. For these processes, an extra set of equations is solved in each grid box. The individual grid boxes interact with their neighbours on all six faces, exchanging temperature, moisture (in the atmosphere, salt in the case of the ocean), and momentum between them.

\subsubsection{Single Column Models}

Global climate models, as the name says, simulate the entire planet. Depending on the process of interest, it might be sufficient, or at least indicative, to choose a single column out of the big model. Single column models are just that, a single column taken from a host GCM. Simulations with a single column, rather than an array of columns, are fast and computationally inexpensive. Of course, large scale processes cannot be investigated with a single column alone. However, providing the model is forced with a representation of these processes, fast small scale processes, especially in the vertical, can be studied in detail. The low costs of such simulation allow for large ensembles, and a wide range of simulations in which parameters are varied. 
Figure 1.8: Schematic of the architecture of a coupled global climate model. Figure from Dippe et al. (2018b)

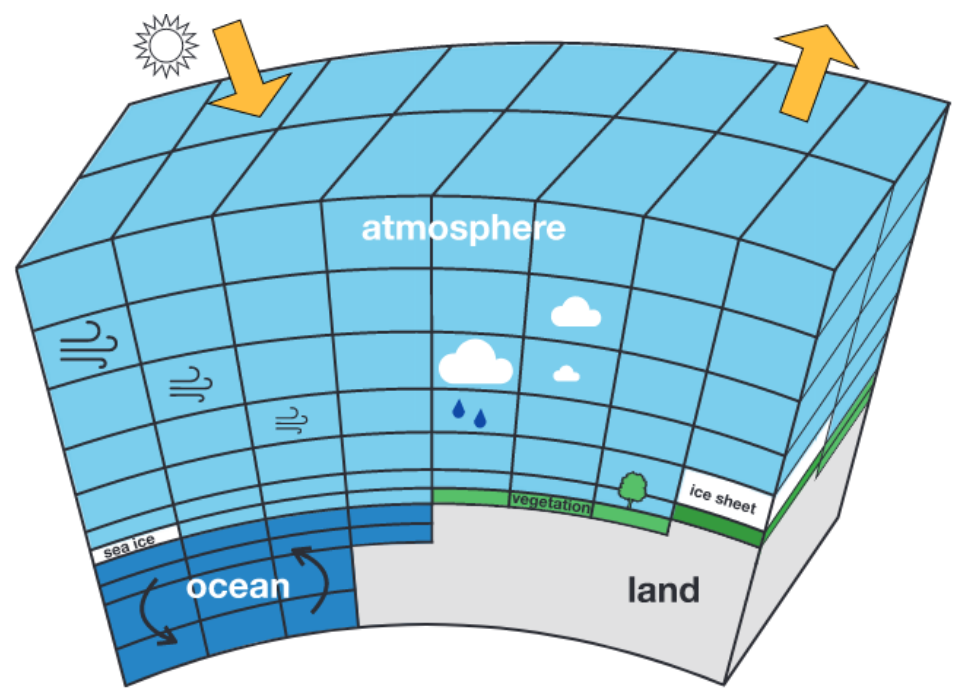

\subsubsection{The CMIP5 Database}

A model cannot be considered an actual accurate representation of the natural world. Numerous assumption have to be made, and the equations governing our surroundings are not known in their entirety. However, if enough models are available, with each their own biases, the statistical average might give a fairly accurate and reliable representation of the natural world. Standardised simulations have been performed with a number of coupled global circulation models within the framework of the Coupled Model Intercomparison Project Phase 5 (CMIP5, (Taylor et al., 2012)). The output of these models has been made available, so that the climate science community can analyse questions of their interest from this large ensemble. The output is also useful for model inter-comparison studies, in which we might address which models perform more reliably and why. A range of different simulations is available to address different scientific questions.

Each model simulates natural variability. The natural model state and variability can be inferred from the pre-industrial control simulation set. In this setting, greenhouse gas forcing is constant throughout the simulation, and is set to pre-industrial values. The GCMs are spun up from initial state to find their equilibrium, and then the simulation is continued for at least 500 years. The length of the simulation allows us to investigate inter-annual processes statistically, as it provides a large sample size.

Another set of simulations includes the radiative forcing effect of increasing greenhouse gases in the atmosphere. These representative concentration pathway scenarios (RCPs) are engineered to simulate the effect of rising greenhouse gas concentrations, aerosols and land use. These simulations start from historical greenhouse gas forcing, and then slowly increase it to achieve a certain additional energy input to the climate system at the end 
of the century. With output from these simulations, we can study the impact of increased greenhouse gas concentrations concentrations on the climate system.

\subsection{In situ observations}

The tropical Atlantic is bordered by two continents whose livelihood is influenced by the local effect of sea surface temperature variations. In order to better understand the patterns of interaction between, for example, the West African Monsoon and the SST off the African coastline, there is a need for reliable observations. To this end, buoys of the Prediction and Research Moored Array in the Tropical Atlantic were set up in 1997 (Bourlès et al., 2008) (Fig 1.9). The buoy array has since been maintained and extended. The array is a source of valuable data for the scientific community. The buoys measure ocean quantities such as temperature, salinity, and currents. Some of the buoys also measure air-sea fluxes such as radiation and precipitation. The measurements in combinations are especially invaluable in the tropics, where the atmosphere and the ocean are strongly coupled. However, with a maximum record length of 20 years, the limited amount of observations does not allow us to investigate processes such as interannual variability. Furthermore, the observations from the buoy array are locally confined. Spatial patterns cannot be determined from them.

Figure 1.9: Location and evolution of the Prediction and Research Moored Array in the Tropical Atlantic. Red squares form the initial backbone of the mooring array, followed by a northeast and a southwest expansion (blue stars and green circles), and the southeast extension (yellow triangle, which is also used in this study). Green crosses mark island locations at which measurements are taken. Figure from Bourlès et al. (2008)

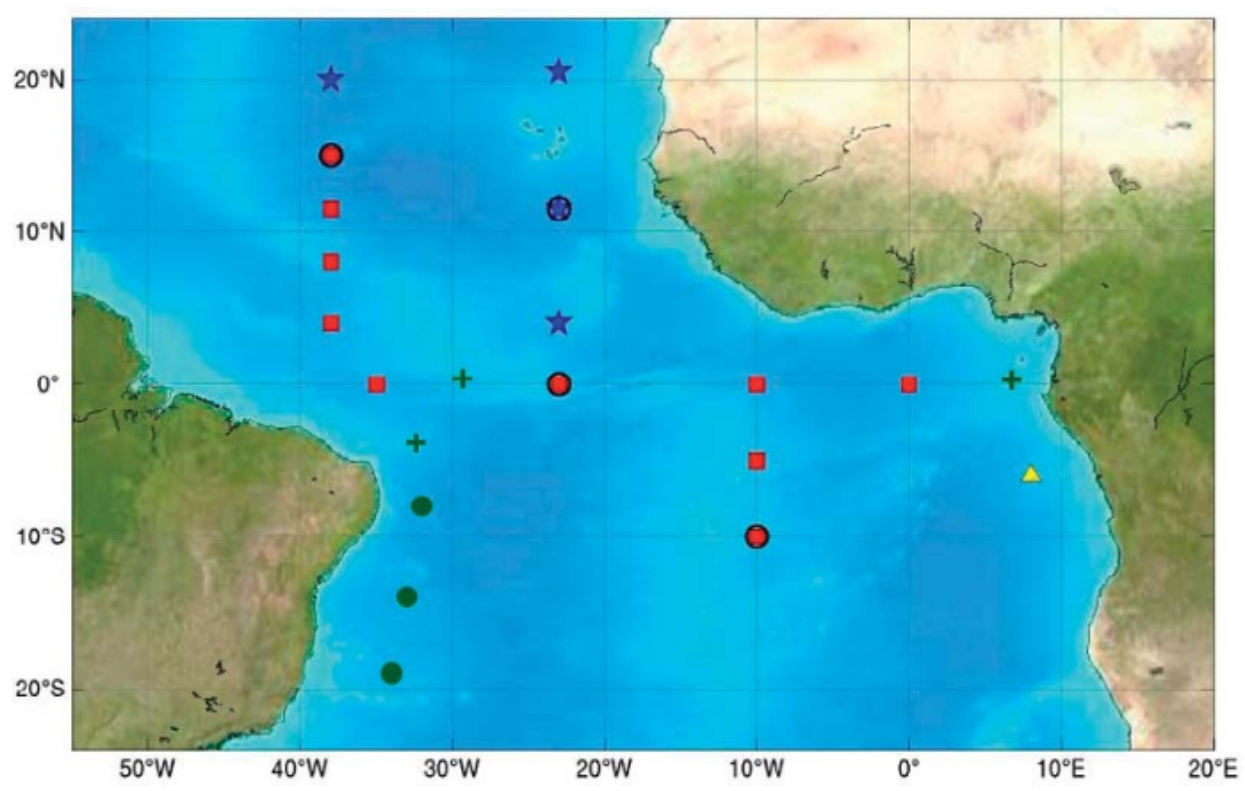




\subsection{Reanalysis data}

In order to span the spatial domain of the tropical Atlantic, we employ a second set of data products to investigate processes in the tropical Atlantic and validate model results. We make use of reanalysis data, which assimilates available in-situ observations into a (global) model. In this way, temporal as well as spatial gaps can be filled in a physically consistent way. The resulting complete data set is limited only by the resolution of the model. While in-situ and remote data are limited by their record length and eventually by experimental uncertainty, reanalysis data also include possible errors due to the assumptions made in the numerical model and data assimiliation methodologies. To use either is a trade-off between accuracy and availability.

In this thesis, we focus on (Global) Coupled Model simulation output, PIRATA mooring data and reanalysis data. ARGO floats or ship based observations are other valuable resources of observational data, but we do not consider them here.

\subsection{Aim and Outline}

In the beginning of this chapter the importance of the tropical Atlantic Ocean to the global climate system, and the region's socio-economic relevance was outlined. Sea surface temperature changes in the tropical Atlantic affect rainfall and droughts on the bordering continents, but also heat waves in Europe and precipitation in North America. Due to the wide ranging effects of the region, it is important to understand Tropical Atlantic variability.

In this thesis we use the tools presented earlier to advance our understanding of nearequatorial sea surface temperature variability in the tropical Atlantic Ocean. Moving from basin wide to smaller scales, we investigate which processes contribute to the observed SST patterns. Considering the discussion about the leading mode of inter-annual summer time SST variability we pose the following research question:

Q1 To what extent does the Bjerknes Feedback contribute to inter-annual SST variability in the Tropical Atlantic?

To address this question we perform statistical time series analysis on reanalysis data in chapter 2. This particular set of reanalysis data had not been used to investigate the Bjerknes Feedback in the Tropical Atlantic. While reanalyses often are our best guess of the physical world, uncertainties about their accuracy remain. It therefore adds confidence to a result if it is obtained from several products. To investigate the air-sea coupled feedback we use the state-of-the art ocean reanalysis system ORA-S4 (Balmaseda 
et al., 2013) from the European Center for Medium Range Weather Forecasts (ECMWF), and its atmospheric counterpart ERA-Interim (Dee et al., 2011) from the same center. We identify the recurring pattern of inter-annual sea surface temperature variability that describes the Atlantic Niño. To determine its relation to the coupled feedback, we crosscorrelate indices for eastern equatorial heat content, sea surface temperature and west equatorial wind stress with the appropriate time lag.

With the knowledge of how the feedback manifests in the physical world, we ask the question:

Q2 How accurate is the representation of the Bjerknes Feedback in state-of-the-art coupled global climate models?

We apply the same metrics we have used on the reanalysis data to the output of climate model simulations. For a comprehensive understanding of intrinsic GCM performance we use the pre-industrial control ensemble from the CMIP5 database. We investigate to what extent the models show variability associated with the Atlantic Niño, and how the variability in the atmosphere and in the ocean is related. We focus on the timing of the connection, and its strength. Using the reanalysis results as a reference, we can infer the attributes that a better-performing GCM has. Our seasonally stratified analysis reveals new insights into the strengths and weaknesses of climate models when simulating tropical Atlantic variability, especially about the timing of the seasonal cycle. Since the latter and the inter-annual variability are closely linked, we ask the question:

Q3 What controls the model deficiencies of the sea surface temperature seasonal cycle?

To answer this question we employ a single column model (SCM) of a state-of-the-art coupled global climate model in chapter 3. The model is a 1D component of the 3D GCM EC-Earth (Hartung et al., 2018; Hazeleger et al., 2012), which follows the architecture of the CMIP6 version of EC-Earth. It consists of an ocean component coupled to an atmosphere component, and closely represent its 3D host. The computational cost-efficient SCM allows us to perform a wide range of sensitivity experiments, which would be costly in the $3 \mathrm{D}$ version. For model validation, we place the SCM at the location of one of the PIRATA moorings, on the south-east extension of the Atlantic cold tongue. This mooring offers high temporal resolution in-situ observations, which best represent the actual state of the ocean, at the surface and below. The combination of the fast SCM and the available in-situ measurements yield valuable insights into the controls on the model state, and where it deviates from reality. Some parts of the models are more error-prone that others, and are therefore more likely to cause biases. Among these are the parametrisations. For some processes, we do not have analytical equations but must describe them based on 
observations and physical reasoning. Turbulent mixing is one of the processes that always has to be parametrised, because it acts on very short time scales and on spatial scales much smaller than a grid box. Knowing that it is difficult to accurately capture turbulent motion, we ask ourselves:

Q4 What is the impact of upper ocean turbulent mixing on the simulation of the tropical Atlantic climate system?

With the single column model, we are able to perform numerous experiments in an attempt to match observed sea surface temperatures. The close similarity between the 1D and the 3D version allows us to transfer the results from the SCM to the GCM, reasonably assuming that the GCM bias results from similar processes as the SCM bias. In chapter 4, we perform sensitivity experiments with the full three dimensional global coupled version of EC-Earth, based on the results of chapter 3. Two simulations represent a potential future climate, following the representative concentration pathway that increases energy input into the troposphere by $8.5 \mathrm{~W} / \mathrm{m}^{2}$ at the end of the century. One of the simulations serves as the control, and is un-altered, while the other features enhanced turbulent vertical ocean mixing. From the difference between the two we can infer the impact of more realistic turbulence parameterisation on the present state simulation, but also on the projected climate change.

Triggered by the large impact the mixing parametrisation asserts on the system, we ask the question what controls turbulent mixing:

Q5 Is turbulent mixing in the ocean impacted by ocean interior processes alone, or if not, to what extent does the atmosphere assert (local) influence?

In chapter 5, we return to in-situ observations and reanalysis data to study the interaction of turbulent processes with the system. We use PIRATA data, supplemented with high temporal resolution reanalyses to estimate what influences turbulent mixing. We perform heat budget analysis for two key regions in the tropical Atlantic, which suffer from large and fast developing sea surface temperatures biases in GCMs. With this, we can draw conclusions on the processes that govern SST evolution in the physical world. We perform statistical analysis on the time series of the contributions to the SST change, and lead-lag correlate them to indicate causalities.

We end with a synthesis of the results obtained in this thesis, and outline possible avenues for future research in chapter 6 . 


\section{Chapter 2}

\section{The Bjerknes Feedback in the Tropical Atlantic}

This chapter is based on:

Deppenmeier, A.-L., Haarsma, R. J., and Hazeleger, W.: The Bjerknes feedback in the tropical Atlantic in CMIP5 models, Climate Dynamics, pp. 1-17, 2016 


\section{Abstract}

Coupled state-of-the-art general circulation models (CGCMs) still perform relatively poorly in simulating tropical Atlantic (TA) climate. To investigate whether lack of airsea interaction might be responsible for their biases, we investigate the Bjerknes feedback $(\mathrm{BF})$ in the TA, the driver of the dominant interannual variability in that region. First, we analyse this mechanism from reanalysis data. Then, we compare our findings to model output from the Coupled Model Intercomparison Project Phase 5 (CMIP5). The feedback is subdivided into three components. The first one consists of the influence of eastern equatorial sea surface temperature anomalies (SST') on zonal wind stress anomalies $\left(\tau_{u}{ }^{\prime}\right)$ in the western basin. The second component is the influence of wind stress anomalies in the western TA on eastern equatorial oceanic heat content anomalies (HC'). The third component is the local response of overlying SST' to HC' in the eastern TA. All three components are shown to be present in ERA-Interim and ORA-S4 reanalysis by correlating the two variables of each component with each other. The obtained patterns are compared to the ones from model output via pattern correlation per component. While the models display errors in the annual cycles of SST, $\tau_{u}$, and $\mathrm{HC}$, as well as in the seasonality of the feedback,the impact of SST' on wind stress and the impact of wind stress on HC' are simulated relatively well by most of the models. This is especially the case when correcting for the error in seasonality. The third component of the BF, the impact of HC' on SST' in the eastern part of the basin, deviates from what we find in reanalysis. Wwe find an influence of HC anomalies on overlying SSTs in the eastern equatorial TA, but it is weaker than in the reanalysis and it is not strongly confined to the equator. Longitude-depth cross sections of equatorial temperature variance and correlation between subsurface temperature anomalies and SST' in the cold tongue region show that flawed simulation and slow adjustment of the subsurface ocean are responsible for this. 


\subsection{Introduction}

In spite of an overall performance improvement in simulating the world's climate, coupled general circulation models (CGCMs) still display large biases in the tropical Atlantic (TA), both in the ocean and in the atmospheric circulation. Most CGCMs are unable to reproduce the observed cold tongue in the eastern part of the TA basin and there are biases in the slope of the equatorial thermocline (Richter and Xie, 2008; Davey et al., 2002; Chang et al., 2007). Furthermore, there are biases in simulating tropical Atlantic variability (TAV).

A prerequsite of simulating TA climate correctly is that the mechanisms that govern TA climate and its variability are well represented in the models. The large annual cycle causes most of the SST variablity in the TA, two other leading modes of interannual to decadal variability have been identified: the zonal and the meridional mode. The zonal mode with a period of two to four years is driven by the Bjerknes feedback (in the following abbreviated as BF) (Ruiz-Barradas et al., 2000; Keenlyside and Latif, 2007; Jansen et al., 2009), whereas the meridional mode on decadal time scales (e. g. Zebiak, 1993; Carton et al., 1996; Xie and Carton, 2004) arises through the wind evaporation sea surface temperature (WES) feedback (Chang et al., 1997). In this paper we will focus on the zonal mode. This mode is one of the major drivers of interannual variability in the TA. Previous studies have identified the BF as driving mechanism of the zonal mode in reanalysis data. Keenlyside and Latif (2007) found the BF to be present in the TA. Jansen et al. (2009) found not only the BF, but also the recharge oscillator to be a dominant driver of TAV. Ding et al. (2010) confirmed the presence of the BF with principal oscillation pattern analysis, and investigated the seasonality of the upper equatorial Atlantic (Ding et al., 2009). A connection between the two modes of variability has been suggested by Foltz and McPhaden (2010), and a connection between the zonal mode and the Benguela Niño has been suggested by Lübbecke et al. (2010). Several studies have investigated the feedbacks in the TA with the help of general circulation models (GCMs) (DeWitt, 2005; Mahajan et al., 2009; Muñoz et al., 2012; Ding et al., 2015).

Most state-of-the-art CGCMs simulate the mean state and variability in the TA poorly (Breugem et al., 2006). This has often been attributed to the westerly wind bias along the equator (Richter and Xie, 2008). Hazeleger and Haarsma (Hazeleger and Haarsma, 2005) show that vertical mixing can play an important role and Li and Xie (2012) propose that tropical-wide SST errors arise from incorrect simulation of cloud cover and thermocline depth. A recent study by Ding et al. (2015) shows that an improved mean state of the TA can lead to better representation of interannual variability. Our study aims to quantify the strength and phase of the air-sea interaction related to the $\mathrm{BF}$ in newest generation climate models, and possibly point to weaknesses in the model representation. We do so by considering different aspects of the feedback and perform statistical analysis on reanalysis and model data. Below, we shortly outline the BF. 
In the warm phase of the zonal mode, positive sea surface temperature anomalies (SST') in the eastern part of the basin cause the zonal winds $\left(\tau_{u}\right)$ in the western part of the TA to weaken. The associated weakening of the zonal pressure gradient allows the thermocline in the eastern basin to deepen and thereby increases the oceanic heat content (HC) in the region. This reduces entrainment of colder subsurface waters into the turbulently mixed surface layer. In addition, the weaker winds may lead to less atmospheric cooling by turbulent surface heat fluxes. The result is a strengthening of the positive SST' in the region, closing the feedback loop. The opposite happens in the cold phase of the zonal mode (Zebiak, 1993).

As a reference for the model results we use a set of reanalysis data that has, to our knowledge, not yet been used for the analysis of the zonal mode. We consider monthly as opposed to seasonal time series because of the pronounced annual cycle in the region. It has been suggested that the zonal mode can be understood as an interannual variation of the strength of the cold tongue (Burls et al., 2012, 2011). This leads to the hypothesis that if the annual cycle is simulated incorrectly, either in timing or strength, the representation and mechanism of the zonal mode will be flawed as well. Therefore, we compare the simulated annual cycles to annual cycles in the reanalysis before analysing the BF components. This allows us to a posteriori correct the model output for the errors in their annual cycles and to assess whether the mechanism driving the zonal mode is correctly represented in other months than in the reanalysis. Finally, we analyse the simulated subsurface ocean and its interaction with SST' as compared to reanalysis.

This paper is structured as follows: in section 2.2 we describe the data and methodology, in section 2.3 we investigate the BF in reanalysis data, and in section 2.4 we compare first the annual cycle (2.4.1), and subsequently the three components of the BF (2.4.2, 2.4.3) to reanalysis data. In section 2.4 .4 we examine subsurface temperature variance and its correlation to SST' in the TA, followed by the conclusions of this study in section 2.5.

\subsection{Data and Methodology}

The reanalysis data used in this study is obtained from the European Centre for MediumRange Weather Forecasts (ECMWF). The variables $\tau_{u}$ and SST are taken from the global atmospheric reanalysis ERA-Interim (Dee et al., 2011), and the potential ocean temperature from the Ocean Reanalysis System 4 (ORA-S4) (Balmaseda et al., 2013). Note that the SST from ERA-Interim and ORA-S4 do not differ significantly, since ERA-Interim was used to drive the ocean reanalysis for 20 years out of the 35 years we use in this study. We use time series from 1979 until 2013, due to availability (ERA-Interim) and trustworthiness of the data (ORA-S4). For the first years of ORA-S4, 1958 until 1979, before the satellite era, little data is available to constrain the reanalysis. 
The model output used in this study is obtained from the Coupled Model Intercomparision Project Phase 5 (CMIP5) (Taylor et al., 2012). The CGCMs as well as the markers indicating them in the comparison plots, and the centers where the simulations were performed are shown in Table 2.1. We use the last 150 years of the pre-industrial control runs to investigate the natural variability in absence of anthropogenic forcing and with minimised model drift. It is noteworthy that the warm SST bias in those simulations is as large as it is. We are comparing the CGCM output to current reanalysis data which already includes the global warming in response to the heightened $\mathrm{CO}_{2}$ forcing. The CGCM output we use was produced with pre-industrial forcing and should therefore be colder than the reanalysis data. All data are interpolated to a $1^{\circ} \mathrm{x} 1^{o}$ grid and linearly detrended using monthly mean time series.

The upper ocean heat content is calculated grid-point-wise by integrating the potential ocean temperature upwards from the $293.15 \mathrm{~K}$ isotherm depth to the surface according to Eq. 2.1.

$$
H C(\text { lat, lon })=c_{p} \cdot \rho \int_{Z_{20}}^{0} T(z, \text { lat }, \text { lon }) \mathrm{d} z,
$$

where we assume a uniform density $\rho$ of $1024.75 \frac{\mathrm{kg}}{\mathrm{m}^{3}}$ and a specific heat capacity $c_{p}$ of $3993 \frac{\mathrm{J}}{\mathrm{kg} \cdot \mathrm{K}}$ for sea water (Website, Accessed: 2015-02-23).

We use the following index regions as indicators for the western TA (wa4, $4^{\circ} \mathrm{N}-4^{\circ} \mathrm{S}$; $40^{\circ} \mathrm{W}-20^{\circ} \mathrm{W}$ ) and the eastern TA (EA $4,4^{\circ} \mathrm{N}-4^{\circ} \mathrm{S}$ and $20^{\circ} \mathrm{W}-10^{\circ} \mathrm{E}$ ), cf. Fig. 2.2. The variables and correlations are spatially averaged over these index regions to obtain a time series or a mean value. Note that for the sample size used (length of the time series) and degrees of freedom of the data, the threshold value for a $95 \%$ significant correlation is 0.35. The signal can be considered significant when the number of gridpoint correlations exceeding that threshold is larger than $5 \%$. Note that the variance that is explained by a correlation of 0.35 is still relatively small.

\subsection{Bjerknes Feedback in Reanalysis Data}

In this section we investigate characteristics of the zonal mode in the TA from reanalysis data. Because of the close connection of the zonal mode to the annual cycle mentioned above, we first examine the annual cycles of SST, $\tau_{u}$, and $\mathrm{HC}$, and subsequently the correlations between the variables.

The strong annual cycle of SST in the basin is evident from Fig. 2.1. During boreal winter direct solar radiation warms the ocean with high intensity, temperatures peak in boreal spring when they reach values larger than $300 \mathrm{~K}\left(27^{\circ} \mathrm{C}\right)$. From May onward the temperature distribution changes: the warm waters retreat north-westwards from the east and a cold tongue forms during boreal summer, spanning the region around the equator 
Table 2.1: Models used in this study, the markers denoting them in the graphics, and the centers where the simulations were run. For more information we refer to (CMIP5 Coupled Model Intercomparison Project, Accessed: 2015-07-16).

\begin{tabular}{|c|c|c|c|}
\hline Number & Marker & Model & Center \\
\hline 1 & $\diamond$ & ACCESS1-0 & CSIRO-BOM \\
\hline 2 & $\diamond$ & ACCESS1-3 & CSIRO-BOM \\
\hline 3 & 0 & bcc-csm1-1 & $\mathrm{BCC}$ \\
\hline 4 & 0 & bcc-csm1-1-m & $\mathrm{BCC}$ \\
\hline 5 & $\triangle$ & CanESM2 & CCCma \\
\hline 6 & 0 & CCSM4 & NCAR \\
\hline 7 & 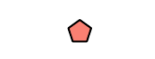 & CESM1-BGC & NSF-DOE-NCAR \\
\hline 8 & $\checkmark$ & CESM1-CAM5 & NSF-DOE-NCAR \\
\hline 9 & 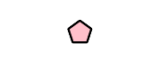 & CESM1-CAM5-1-FV2 & NSF-DOE-NCAR \\
\hline 10 & $\Delta$ & CESM1-FASTCHEM & NSF-DOE-NCAR \\
\hline 11 & 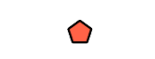 & CESM1-WACCM & NSF-DOE-NCAR \\
\hline 12 & $\mathbf{\square}$ & CMCC-CESM & $\mathrm{CMCC}$ \\
\hline 13 & $\square$ & CMCC-CM & $\mathrm{CMCC}$ \\
\hline 14 & $\square$ & CMCC-CMS & $\mathrm{CMCC}$ \\
\hline 15 & & CNRM-CM5 & CNRM-CERFACS \\
\hline 16 & & CSIRO-Mk3-6-0 & CSIRO-QCCCE \\
\hline 17 & $x$ & FIO-ESM & $\mathrm{FIO}$ \\
\hline 18 & $\diamond$ & GFDL-CM3 & NOAA GFDL \\
\hline 19 & $\diamond$ & GFDL-ESM2M & NOAA GFDL \\
\hline 20 & $\triangleleft$ & GISS-E2-H & NASA GISS \\
\hline 21 & $\triangleleft$ & GISS-E2-H-CC & NASA GISS \\
\hline 22 & $\triangleleft$ & GISS-E2-R & NASA GISS \\
\hline 23 & $\triangleleft$ & GISS-E2-R-CC & NASA GISS \\
\hline 24 & 0 & HadGEM2-CC & $\mathrm{MOHC}$ \\
\hline 25 & 0 & HadGEM2-ES & MOHC-INPE \\
\hline 26 & O & IPSL-CM5A-LR & IPSL \\
\hline 27 & ○ & IPSL-CM5A-MR & IPSL \\
\hline 28 & 0 & IPSL-CM5B-LR & IPSL \\
\hline 29 & 0 & MIROC-ESM & MIROC \\
\hline 30 & 0 & MIROC-ESM-CHEM & MIROC \\
\hline 31 & $\triangleright$ & MPI-ESM-LR & MPI-M \\
\hline 32 & $\triangleright$ & MPI-ESM-MR & MPI-M \\
\hline 33 & $\triangleright$ & MPI-ESM-P & MPI-M \\
\hline 34 & $\Delta$ & MRI-CGCM3 & MRI \\
\hline 35 & $\diamond$ & NorESM1-M & $\mathrm{NCC}$ \\
\hline 36 & $\diamond$ & NorESM1-ME & $\mathrm{NCC}$ \\
\hline
\end{tabular}



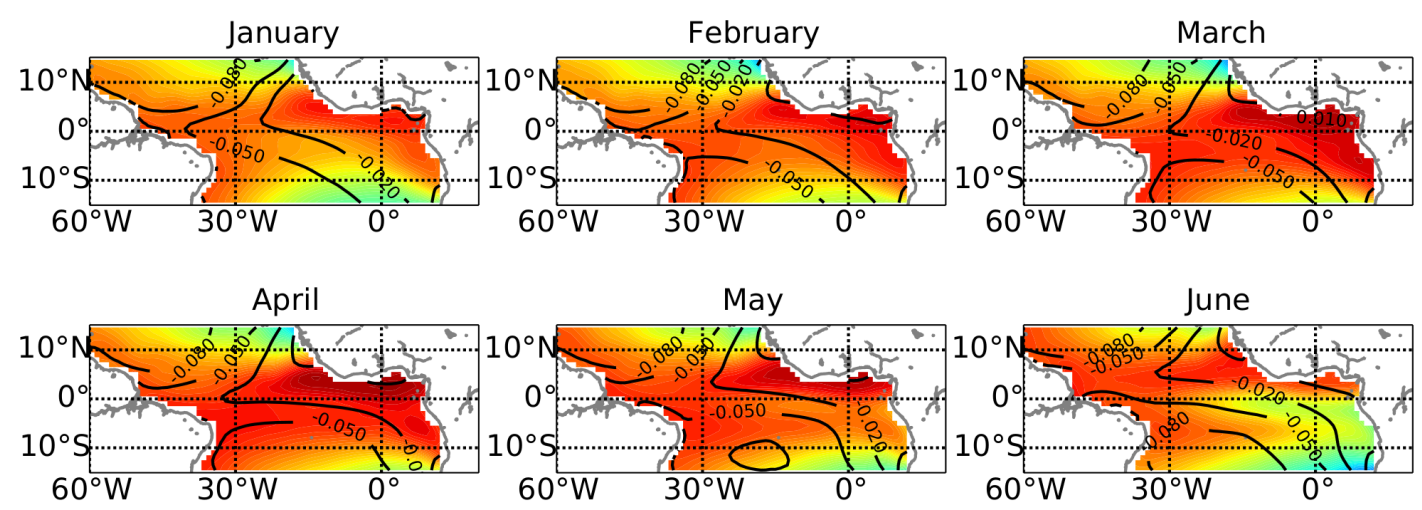

300
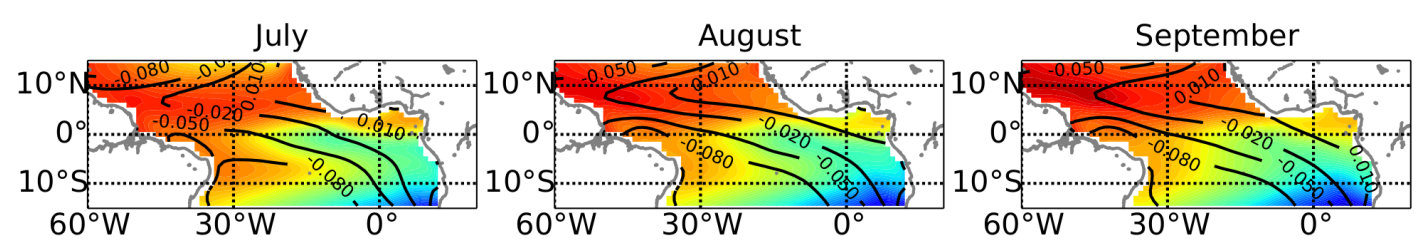

302

298
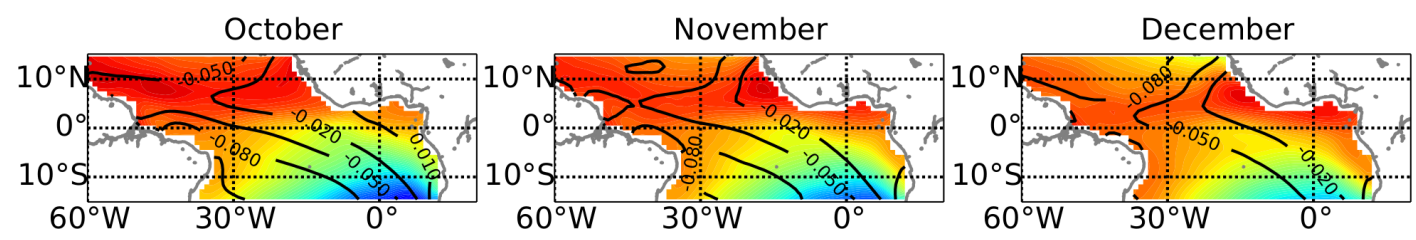

292

Figure 2.1: Climatology obtained from ERAInterim reanalysis of SST $[\mathrm{K}]$ and $\tau_{u}\left[\frac{N}{m^{2}}\right]$ in the TA throughout the year.

and covering the whole eastern to central part of the basin. This cooling is thought to be caused by upwelling of cold water in the eastern part of the basin, which is closely linked to the sudden onset of the West African Monsoon (Mitchell and Wallace, 1992). With strengthening of the westward winds and cooling of the SST the BF becomes active (Burls et al., 2011). The cold tongue is most prominent in August. From September onward, upwelling is reduced through weakening of the southerly cross-equatorial winds, and the cold tongue retreats.

The cold tongue region displays high SST variability on both annual and interannual time scales. Correlating monthly SST time series of the chosen cold tongue index EA4 to the monthly time series of SST over the whole basin yields the pattern of the interannual mode of SST variability, the zonal mode. Performing regression analysis in the same manner delivers information about the strength of this mode of variability. Both are shown in Fig. 2.2.

The BF consists of three components: the influence of SST anomalies in the eastern part of the equatorial Atlantic basin on $\tau_{u}\left(\lambda_{S S T \rightarrow \tau_{u}}\right)$, the effect of $\tau_{u}$ anomalies in the west on the east-equatorial $\mathrm{HC}\left(\lambda_{\tau_{u} \rightarrow H C}\right)$, and the local effect of the $\mathrm{HC}$ anomalies on overlying SSTs $\left(\lambda_{H C \rightarrow S S T}\right)$ in the cold tongue region. The connection between the two variables of the three components can be illustrated by correlating the anomaly time series to 


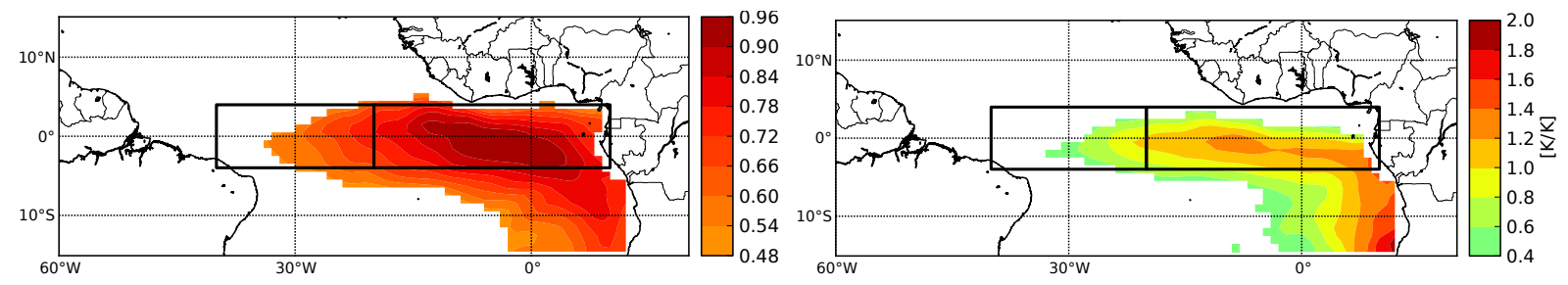

Figure 2.2: The zonal mode obtained from ERA-Interim averaged over June, July and August. Left: correlation between monthly SST in EA4 (right box), and SST elsewhere in the basin. The left box indicates the wa4 index used for other correlation analysis later on. Right: linear regression analysis of monthly $\mathrm{SST}^{\prime}{ }_{E A 4}$ on monthly SST' elsewhere.

another according to Eq. 2.2, where $\sigma_{a}$ and $\sigma_{b}$ are the standard deviations of variable $a$ and $b$, respectively, and primes denotes anomalies.

$$
r(\text { lat }, \text { lon })=\frac{\sum a^{\prime}(t) \cdot b^{\prime}(t, \text { lat, lon })}{\sigma_{a} \cdot \sigma_{b}}
$$

Appropriate indices are chosen to represent the eastern and the western basin (see Fig. 2.2 and section 2.2) for variable $a$. In order to detect the separate components of the BF, we adapt a method similar to (Keenlyside and Latif, 2007). For each component of the feedback we correlate the spatially averaged timeseries of anomalies in the region of interest of variable $a$ point-wise to the time series of variable $b$. Each of the interactions is investigated seperately for every calendar month. For the first component of the BF, $\lambda_{S S T \rightarrow \tau_{u}}$, we average SST' over the box EA4 (see Fig. 2.2). The box was chosen to be slightly larger than reported in previous literature in order to avoid constraining the model output too much. In Fig. 2.3 the correlations between SST' and $\tau_{u}$ ' are shown for each month of the year. Because the response time of the atmosphere to SST' is less than a month, we use a zero month lag for $\lambda_{S S T \rightarrow \tau_{u}}$.

A significant correlation associated with the first component of the BF appears in March, broadens in April, and is strongest in the western Atlantic along the equator in May. The equatorial response of $\tau_{u}$ to SST' is extends to a large area north and south of the equator. In June the area of highest correlation departs from the equator, indicating that another mechanism, possibly connected to the migrating ITCZ becomes more important. In July, the area with maximum correlations has moved northward together with the ITCZ. The second component of the BF, $\lambda_{\tau_{u} \rightarrow H C}$, describes the impact of wind stress anomalies in the western part of the basin on the eastern equatorial oceanic HC. $\tau_{u}$ ' in the west generate Kelvin waves that propagate eastward along the equatorial waveguide and cause a deepening of the thermocline in the east, thereby increasing the upper ocean HC. Due to the time it takes a Kelvin wave to travel from east to west we introduce a lag of one month when correlating the two variables in order to exclude direct influences of $\tau_{u}$ on $\mathrm{HC}$ via an atmospheric pathway. To identify $\lambda_{\tau_{u} \rightarrow H C}$, we average $\tau_{u}$ over the box wa4 (see Fig. 2.2) and correlate the anomalies to the $\mathrm{HC}^{\prime}$ ' in a point-wise manner. 

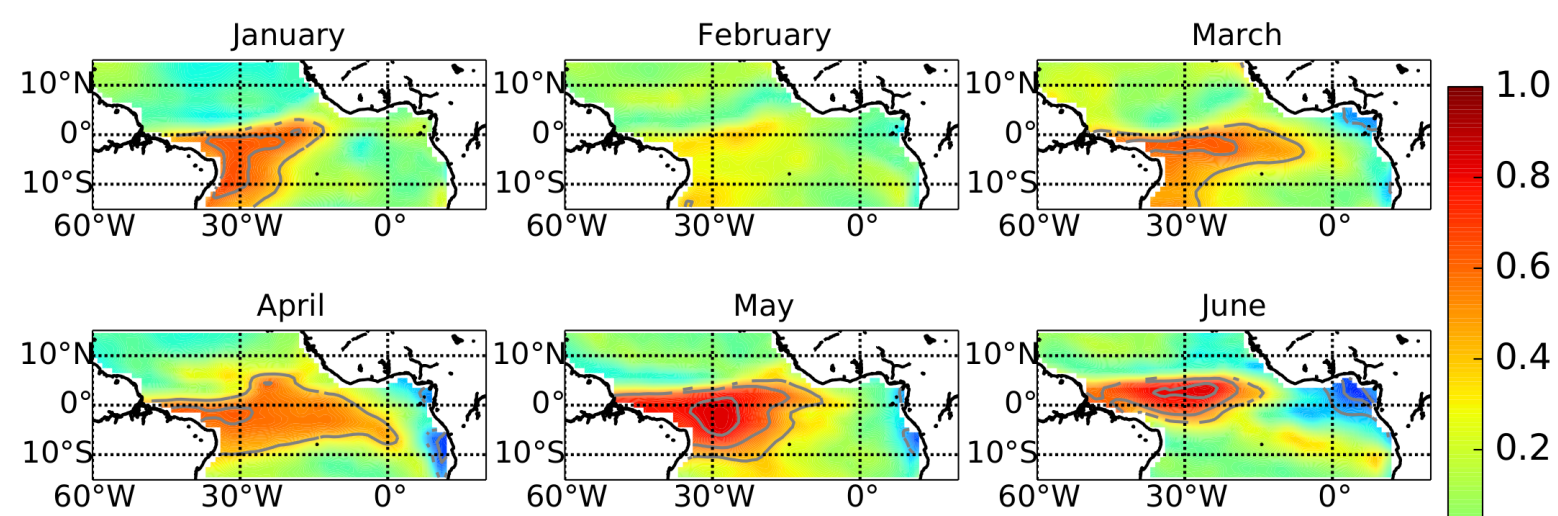

0.4

0.2
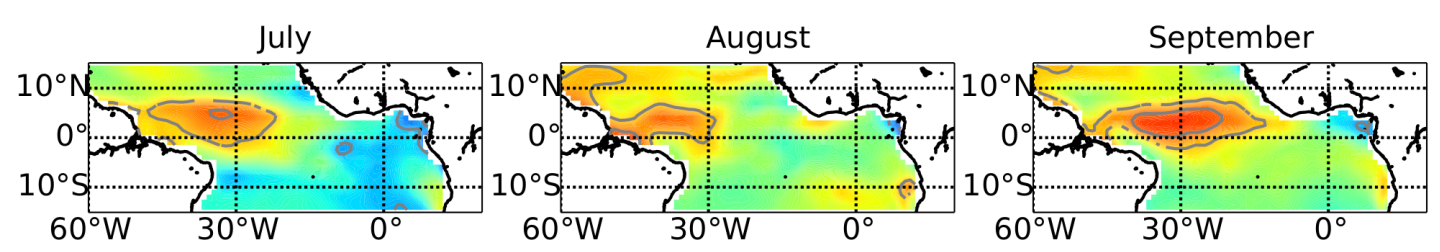

$0.0 \mathrm{R}$
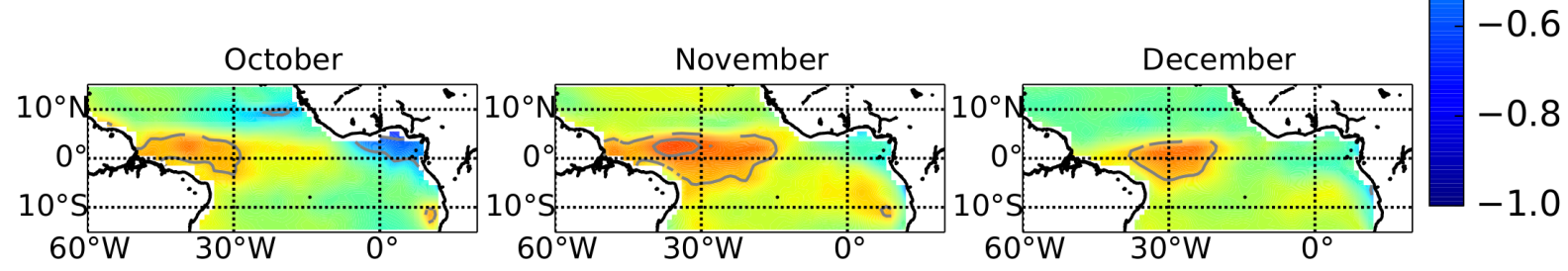

Figure 2.3: Correlation pattern obtained from reanalysis (ERA-Interim) between SST anomaly in EA4 and wind stress anomalies for each month of the year, the first component of the BF $\lambda_{S S T \rightarrow \tau_{u}}$. Contours indicate significant correlation at the $90 \%$ confidence level.

While testing the sensitivity of the results to the chosen lag, we observe that the highest correlation between $\tau_{u}$ ' and $\mathrm{HC}$ ' with a BF like pattern occurs at zero month lag in the reanalysis data, although the results are very similar to a one month lag. We hypothesise that other mechanisms than the BF, such as Ekman pumping, can be responsible for this, and therefore retain the one month lag.

The monthly results are shown in Fig. 2.4. We notice a build up of high correlation centered on the equator in March-April and a further increase in April-May. The correlation weakens in May-June, and fades into a pattern that is reminiscent of a discharge process in June-July. The timing of the correlation and its maximum strength in May coincides with that of $\lambda_{S S T \rightarrow \tau_{u}}$, suggesting that the two processes are closely connected. In July, the pattern clearly deviates from the expected $\lambda_{\tau_{u} \rightarrow H C}$ pattern associated with the BF. This is also the case for $\lambda_{S S T \rightarrow \tau_{u}}$, and indicates the end of the season when the BF is active.

The third component of the BF describes the influence of oceanic HC on overlying SST', $\lambda_{H C \rightarrow S S T}$; mainly to be seen in the cold tongue region where the thermocline is shallow and allows cold subsurface waters to mix with the warm surface waters (Fig. 2.5). This is 

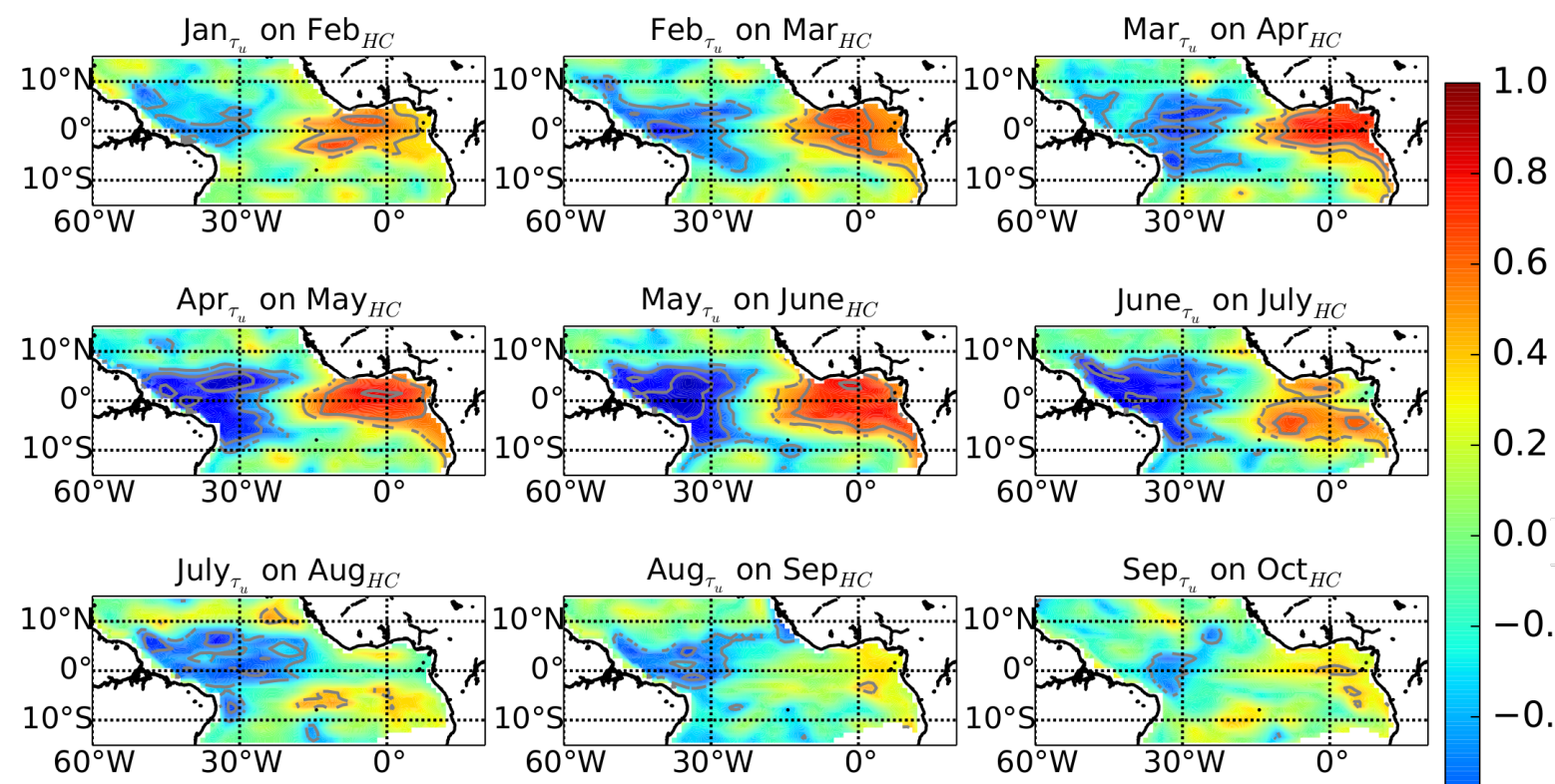
$0.0 \mathrm{R}$
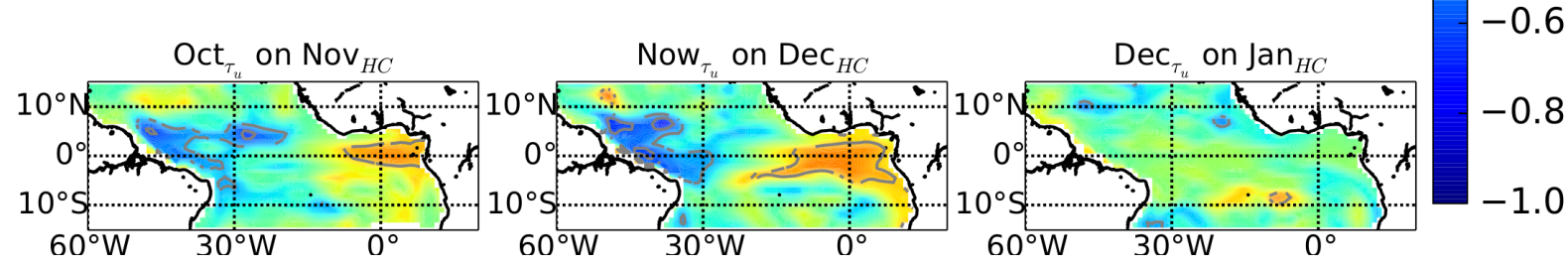

Figure 2.4: Correlation pattern obtained from reanalysis (ERA-Interim) between $\tau_{u}$ anomaly (ERA-Interim) in WA4 and upper ocean HC anomalies (derived from ORA-S4) elswhere in the basin for each month of the year, the second component of the BF $\lambda_{\tau_{u} \rightarrow H C}$. Contours indicate significant correlation at the $90 \%$ confidence level.

the least well-defined of the three components, displaying correlations between subsurface anomalies and SST' in several regions of the basin. Focussion on the equator, the correlation increases as early as April, albeit very weakly at this stage. The signal peaks in May, and persisits throughout June. In the latter month, the signal is still located almost symmetrically along the equator, which is hardly the case for $\lambda_{S S T \rightarrow \tau_{u}}$. The signal quickly vanishes afterward, consistent with the timing of the other two components which have also begun to cease by that time. There is also a significant correlation signal in November up to January, indicative of the second peak of the zonal mode described by Okumura and Xie (Okumura and Xie, 2006). Signs of this can also be found in the other two components of the BF loop shown above.

From the monthly stratified correlation analysis of reanalysis data we conclude that the BF mechanism indeed exists in the TA, confirming earlier studies. The activity of the three components coincides in May and June, when distinct correlation patterns are visible. Zonal wind stress anomalies originate from SST' early in the year (March, April). In these months, $\tau_{u}$ ' induce oceanic perturbations in the western part of the TA that travel 

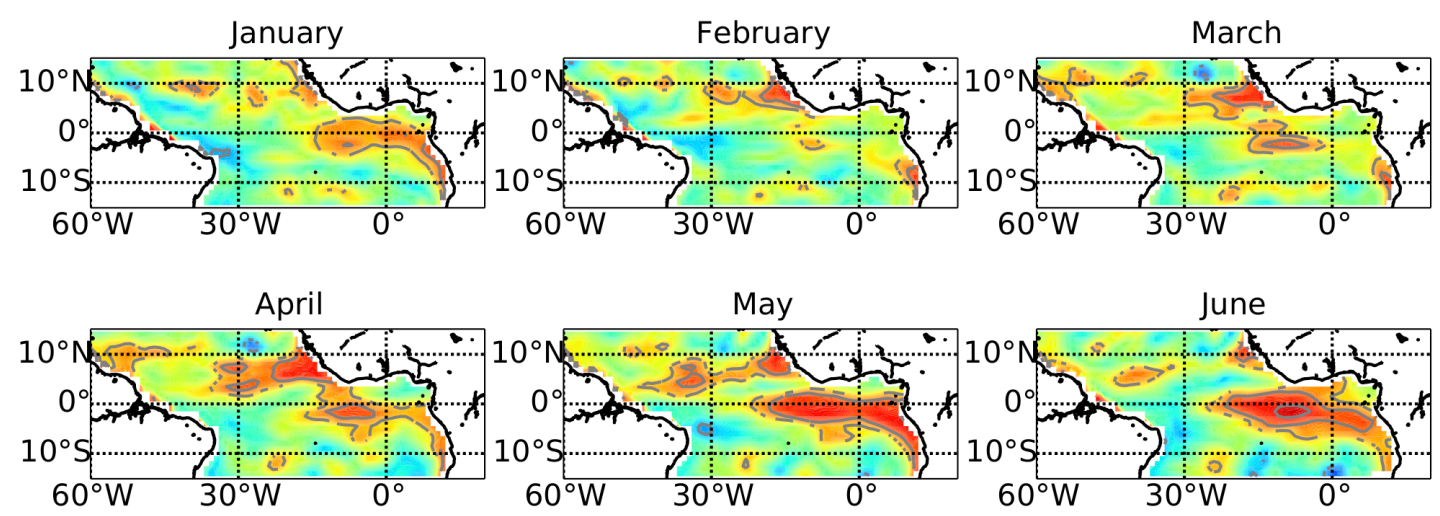

0.6

0.8

0.4

0.2
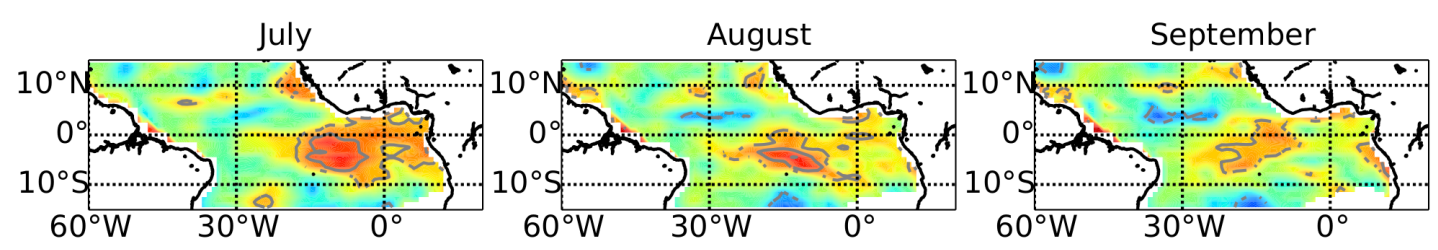

$0.0 \mathrm{R}$
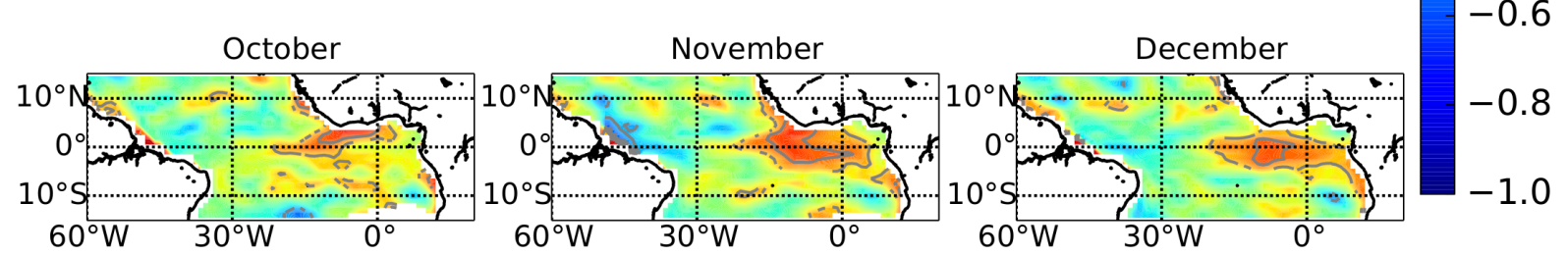

Figure 2.5: Correlation pattern obtained from reanalysis between upper ocean $\mathrm{HC}$ anomalies (derived from ORA-S4) and SST anomalies (ERA-Interim), correlated pointwise, component three of the BF $\lambda_{H C \rightarrow S S T}$. Contours indicate significant correlation at the $90 \%$ confidence level.

to the east of the basin. There, the ocean stratification reaches a state in May in which the $\lambda_{H C \rightarrow S S T}$ coupling is possible. The resulting SST' again affect the atmospheric circulation along the equator, impacting $\tau_{u}$ '. During these months the feedback loop is active. The equatorial atmosperic response decreases in June, when the atmosphere is influenced by other factors, most importantly the migration of the ITCZ. Remnants of the feedback loop can be observed in July, although other mechasnisms seem to more dominant at this point.

In contrast to other studies about the BF, we show a monthly stratified picture of the BF. It becomes clear that the signal is not always centered on the equator throughout boreal summer. Other processes seem to mix with the BF leading to correlation patterns that deviate from the one found by Keenlyside and Latif (2007). Their study also analyses the seasonality of the BF. Our results are in line with the authors' finding that $\lambda_{S S T \rightarrow \tau_{u}}$ is mostly active in boreal spring and early boreal summer, and that $\lambda_{H C \rightarrow S S T}$ is mostly active in late boreal spring and early summer. The regression analysis in Keenlyside and Latif (2007) indicates a coupling until as late as August, which we cannot confirm, as the signal is no longer centered on the equator in our analysis. While the tropical Atlantic 
atmosphere is still sensitive to SST' in the east, the BF loop is not completed by an equatorial Kelvin wave. In the reanalysis data set analysed here the oceanic response of $\mathrm{HC}$ to $\tau_{u}$ ' is also subject to seasonal modulation. The signal on the equator is most prominent in boreal spring until early boreal summer.

We have demonstrated that it is important to look at the monthly stratified picture of the tropical Atlantic when investigating the $\mathrm{BF}$, as the correlation patterns of the three components vary strongly between the months. We continue to examine the CGCMs performance in the months May, June, and July. During May and June the correlation patterns are most distinct, especially for $\lambda_{S S T \rightarrow \tau_{u}}$ and $\lambda_{H C \rightarrow S S T}$. In July the correlation strength weakens and the pattern changes, indicating the end of the BF.

\subsection{Comparison between Reanalysis and Model Out- put}

\subsubsection{Annual cycle}

In the former section we have established that a BF exists in the equatorial Atlantic ocean, confirming other studies (Keenlyside and Latif, 2007; Okumura and Xie, 2006). It is strongest in the early boreal summer months May and June and fades in July. In the following we will compare the model output to reanalysis data in order to investigate whether the models capture the BF. Because of the strong seasonality and the phaselocking to the annual cycle we will first compare the annual cycle of the reanalysis to those of the models, as shown in Fig. 2.6. The three variables that are related to the BF, SST, $\tau_{u}$, and $\mathrm{HC}$, are examined in the regions of interest for the BF, i. e., EA4 for SST and $\mathrm{HC}$, and wa4 for $\tau_{u}$.
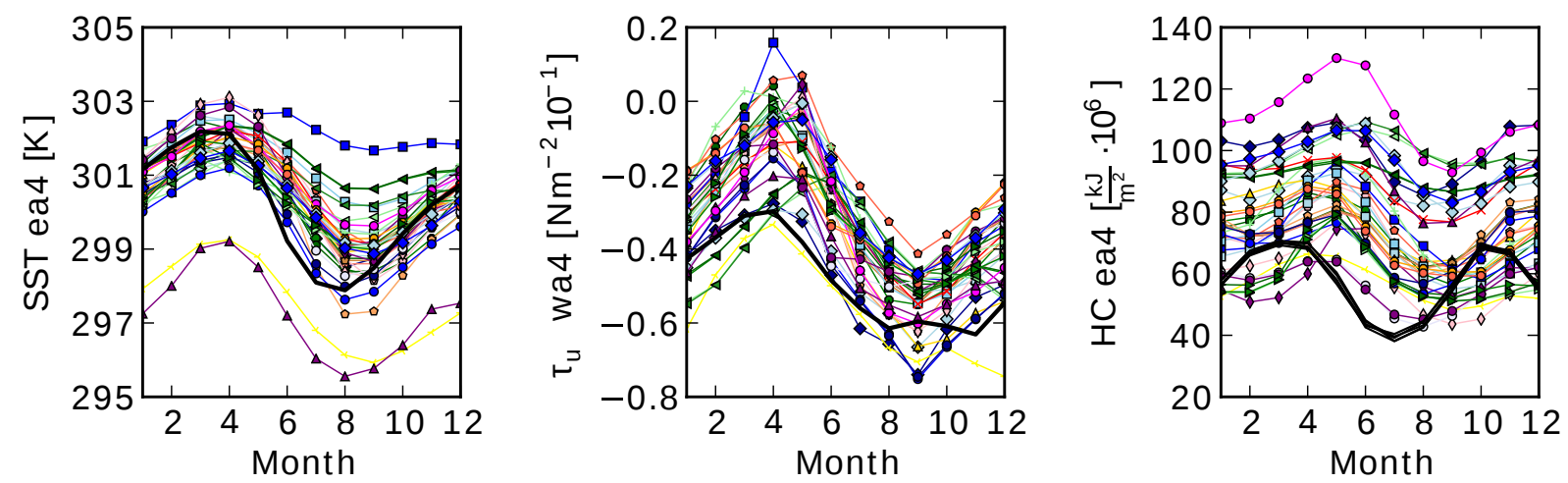

Figure 2.6: Seasonal cycles of the variables relevant to the BF: SST in EA4, $\tau_{u}$ in WA4, and upper ocean HC in EA4. Reanalysis data is plotted in black, for reference of the individual models see Table 2.1 . 
In all three panels of Fig. 2.6 there are obvious deviations of the model output from reanalysis, and there is a large spread between the models. For SST (left panel of Fig. 2.6) the models simulate the annual cycle reasonably well during boreal winter and early spring. The model ensemble mean almost coincides with reanalysis from November to April. This implies an overall warm bias in the CGCMs, as pre-industrial control simulations are used. From May onward the reanalysis SST cools strongly, generating a slope that none of the models reproduces. In the following months the models' SST lag behind reanalysis SST, reaching their minimum SST one to two months later than in the reanalysis data. Another feature in the CGCMs' SST annual cycle is their relatively weak amplitude, especially during boreal summer and fall. The weak cooling of eastern equatorial SST is associated with the models' inability to reproduce the boreal summer cold tongue.

The annual cycle of $\tau_{u}$ in the western part of the basin displays similar shortcomings (center panel of Fig. 2.6). Overall, $\tau_{u}$ is markedly underestimated by almost all CGCMs under investigation. None of the examined CMIP5 models simulate the weakening of the winds in September and the following strengthening in November associated with the second zonal mode (second Niño, (Okumura and Xie, 2006)). In the reanalysis $\tau_{u}$ reaches its minimum in April, while several models simulate $\tau_{u}$ at its weakest in May, some even in June. $\tau_{u}$ in the reanalysis reaches its first local maximum in August, while most of the models simulate strengthening until September. This is in agreement with the lag we also find for the minimum of $\tau_{u}$, but is also connected to the missing weakening of $\tau_{u}$ in September as described above.

The oceanic HC displays even larger differences in seasonality with respect to the reanalysis than $\tau_{u}$ and SST. In addition to the large spread between the models, the absolute value of the $\mathrm{HC}$ is up to twice as large as that of the ocean reanalysis and there is a distinct lag in annual cycle of the CGCMs with respect to the reanalysis. The seasonal minimum of the HC is clearly misplaced by up to three months and the models fail to capture the details of the biannual characteristic. Note that the similarities in the annual cycle of $\mathrm{HC}$ shown here and the annual cycle of $\mathrm{Z}_{20}$ as shown by Richter (Fig. 6 in (Richter and Xie, 2008)) demonstrate that $\mathrm{HC}$ is a valid measure for the oceanic subsurface condition as indicated by $\mathrm{Z}_{20}$.

In this section, large errors in the annual cycle for each of the three variables that are involved in the BF have been shown, confirming earlier studies, e. g., (Richter and Xie, 2008; Richter et al., 2014b). We continue to show the annual cycle of variances for each of the three variables.

In Fig. 2.7 shortcomings of the models are distinctly visible. For example, for the variance of $\tau_{u}$ (center panel) we find that the reanalysis data displays a plateau of high variance from April to May, when the winds strengthen. This is also the time when the atmosphere at the equator reacts most strongly to SST' in the east. Most of the models display a single peak which occurs about one to two months later in the year than observed. This 

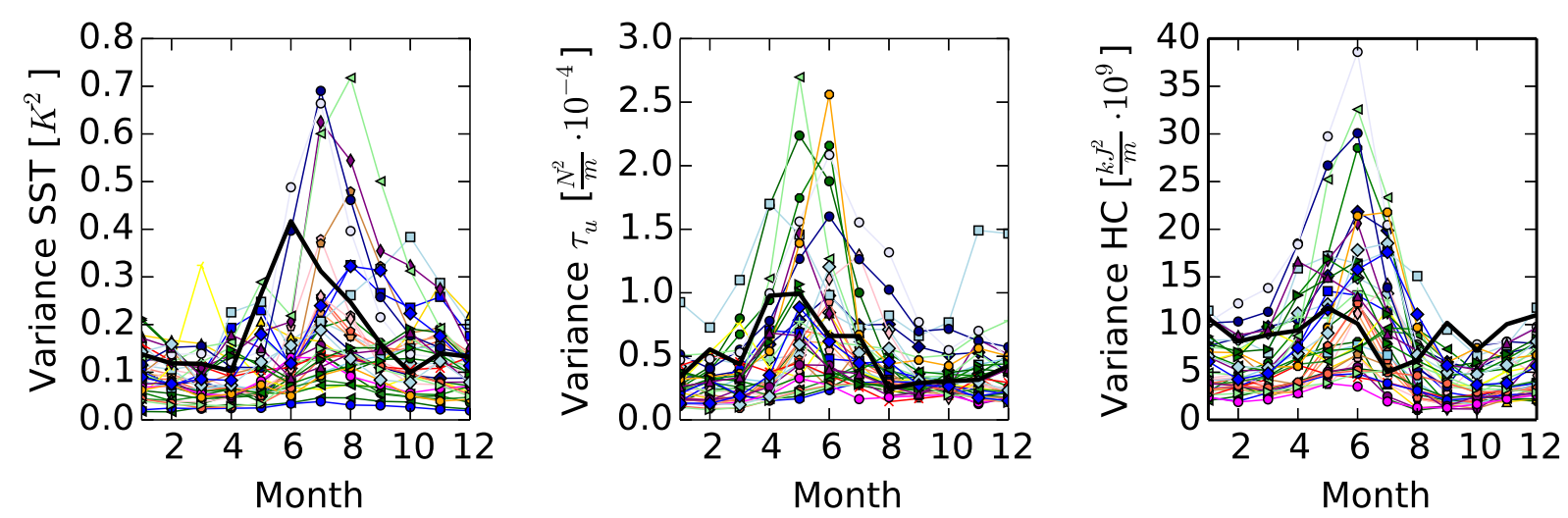

Figure 2.7: Seasonal cycles of variance of SST in EA4 $\left[\mathrm{K}^{2}\right], \tau_{u}$ in WA4 $\left[\mathrm{N}^{2} \mathrm{~m}\right]$, and upper ocean $\mathrm{HC}$ in EA4 $\left[\frac{\mathrm{kJ}}{\mathrm{m}} \cdot 10^{9}\right]$. Reanalysis values plotted in black.

lagged relationship also appears in the annual cycles of variance for SST and HC. Apart from the lag, there are also large errors in the amplitude of variance in CGCMs compared to the variance in reanalysis. These errors are both positive and negative.

For the reanalysis data, the time frame when the $\mathrm{BF}$ is active coincides with the maximum variance of the relevant variables, especially in case of $\tau_{u}$. SST variance steeply increases in May and peaks in June, when the BF loop is closed. We have found biases in the CGCMs' annual cycles, but does this also imply errors in the simulated BF? This could be expected fom the result that the BF is only active in a particular season in the reanalysis: it needs a specific background state to operate. We investigate this question by correlating the three variable pairs obtained from model output in the same manner as done for the reanalysis, and subsequently compare the spatial response patterns to the pattern obtained from reanalysis by pattern correlation analysis.

\subsubsection{Pattern Correlation Analysis}

To investigate whether a model reproduces the $\mathrm{BF}$ we correlate the time series of variable $a$ and $b$ with each other to obtain their coupling pattern as shown for the reanalysis (see Eq. 2.2). Subsequently, we perform pattern correlation between the model and the reanalysis correlation field, according to Eq. 2.3. Here, $r_{r a}$ and $r_{m}$ are the correlation coefficient fields from correlating the variables $a$ and $b$ from the reanalysis and model output, respectively. $\sigma_{r_{r a}} \cdot \sigma_{r_{m}}$ is the product of the standard deviations of the reanalysis and model correlation fields. Prime denotes anomalies as in Eq. 2.2.

$$
M R A=\frac{\sum r_{r a}^{\prime}(\text { lat }, \text { lon }) \cdot r_{m}^{\prime}(\text { lat }, \text { lon })}{\sigma_{r_{r a}} \cdot \sigma_{r_{m}}}
$$

A model that reproduces the coupling pattern yields a high pattern correlation value. We call this value "model-reanalysis agreement" (MRA), it is plotted on the y-axis of 
the multi-model comparison plots (Figs. 2.8 and 2.9). Note that the MRA depends only on the spatial pattern of the response, and not on its correlation strength. Hence much weaker $a-b$ correlation values in the model can still score highly on the MRA axis. To take the correlation between the variable pair into account, we plot the correlation value spatially averaged over the region of interest (i. e., wa4 for $\lambda_{S S T \rightarrow \tau_{u}}$, and EA4 for $\lambda_{\tau_{u} \rightarrow H C}$ and $\left.\lambda_{H C \rightarrow S S T}\right)$ on the x-axis. The original correlation value obtained from the reanalysis is marked as a red line. The ensemble mean correlation strength between the two variables under investigation is marked by a pink line.
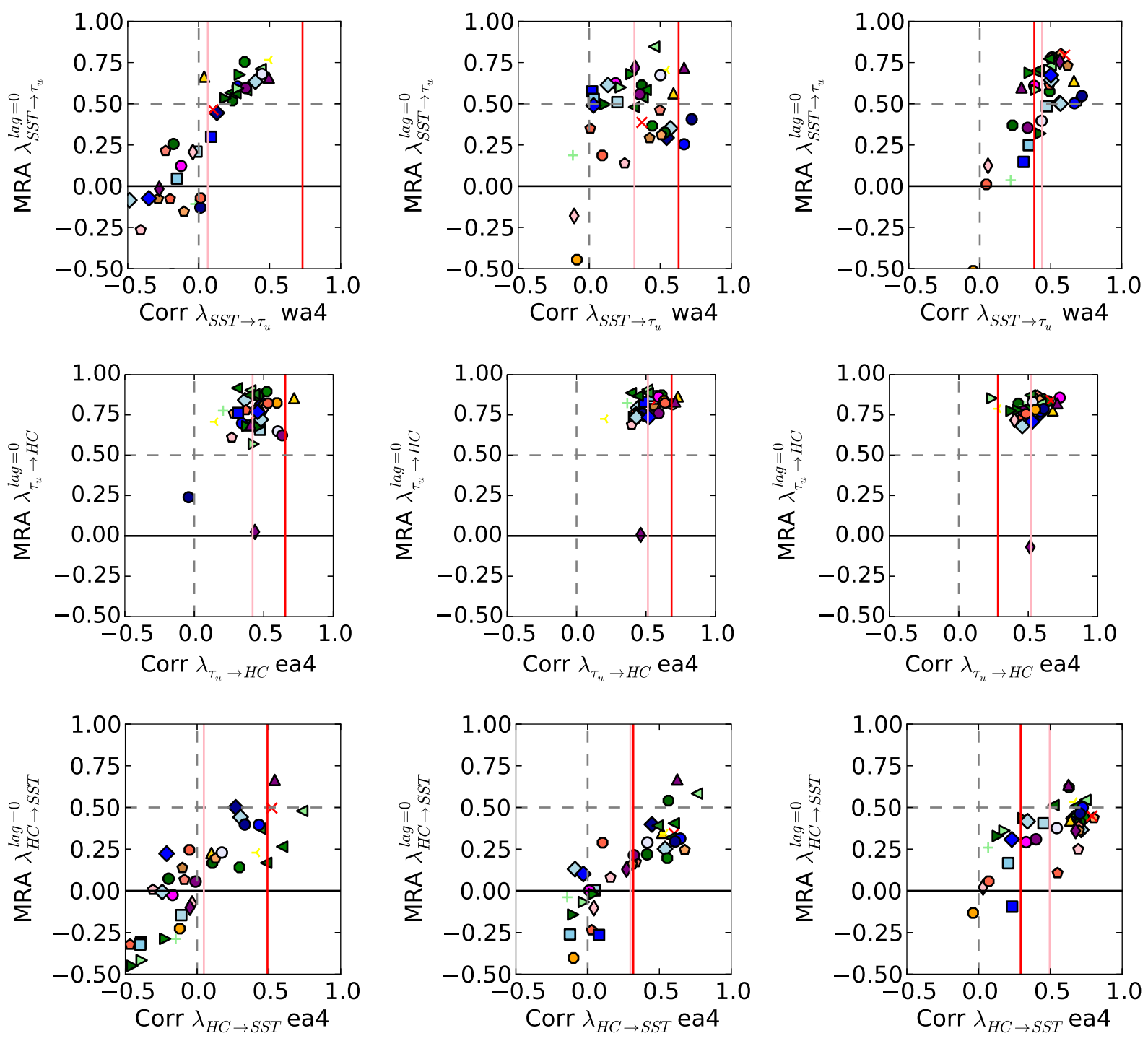

Figure 2.8: Pattern correlation between models and reanalysis (MRA) plotted against the correlation value between the two variables of the respective component of the BF averaged over the area of interest. The red line denotes the reanalysis correlation value in the region, the pink line the multi model average. The three columns show the results for May, June and July individually (April on May, May on June, and June on July in the case of $\lambda_{\tau_{u} \rightarrow H C}$ ).

For the first component of the feedback, $\lambda_{S S T \rightarrow \tau_{u}}$ (first row), we see an improvement in 
model performance of $\lambda_{S S T \rightarrow \tau_{u}}$ from May to June (Fig. 2.8). In May (first row, left panel) the ensemble mean does not simulate the BF, only a few models are located in the upper right of the plot, which means that those CGCMs show some agreement with the reanalysis in both pattern and $\lambda_{S S T \rightarrow \tau_{u}}$ correlation strength. However, none of the models reaches the inter-variable correlation strength obtained from reanalysis. In June (first row, center panel) the overall pattern correlation value increases compared to May, along with an increase in the correlation strength of $\lambda_{S S T \rightarrow \tau_{u}}$. The ensemble mean correlation strength moves closer to the observed one and more models display large pattern agreement with the reanalysis. In July (upper right) the picture is improved again, now most of the models under investigation agree with the reanalysis. Note that in this month the reanalysis pattern of $\lambda_{S S T \rightarrow \tau_{u}}$ does not consist of the pure BF pattern anymore, because the atmosperic response to SST' has moved towards the north.

The second component of the feedback, $\lambda_{\tau_{u} \rightarrow H C}$, is simulated better by the CGCMs. Throughout the three months in boreal summer we investigate, high model-reanalysis agreement (MRA) is obtained. The correlation between $\tau_{u}$ and HC is only slightly underestimated and especially in June nearly all models simulate this component of the feedback loop correctly. In July we observe higher $\tau_{u} \rightarrow H C$ correlations in the EA4 region than in the reanalysis.

The influence of HC' on overlying SST' at the equator $\left(\lambda_{H C \rightarrow S S T}\right)$ is simulated less accurately. The spatial pattern of this BF component is poorly represented throughout the whole season we investigate. Similarly to $\lambda_{S S T \rightarrow \tau_{u}}$, the MRA scores lowest in May and improves slightly with increasing $\lambda_{H C \rightarrow S S T}$ correlation strength in June. Only very few models reach high MRA, even though the correlation strength between HC' and SST' almost reach reanalysis values. As for the other components, the multi-model mean variable correlation strength exceeds the reanalysis value in July, but in this case MRA has not increased significantly. The low MRA shows that, while the CGCMs display an interaction almost as strong as in the reanalysis in the eastern part of the basin, the response pattern is different from the one in the reanalysis. Strong correlations between HC' and SST' are simulated in locations that are absent in the reanalysis and vice versa. This could be due to the fact that we compare basinwide patterns. However, when we restrict the area we use for the pattern correlation to the eastern basin the model-reanalysis agreement is only marginally increased. This indicates that the low MRA also arises from the discrepancy in the spatial representation of $\lambda_{H C \rightarrow S S T}$ in the cold tongue region. The same holds for the $H C \rightarrow S S T$ correlation strength on the x-axis if we choose a smaller index region $\left(2^{\circ} \mathrm{N}-\right.$ $2^{\circ} \mathrm{S}, 20^{\circ} \mathrm{W}-10^{\circ} \mathrm{E}$, not shown). Our conclusion is therefore that the third component of the $\mathrm{BF}$ is the one represented worst with respect to the spatial structure out of the three components of the BF.

In the following we investigate whether the incorrectly simulated annual cycle is responsible for errors of the simulated BF. Most models display lags of variance by one to two 
months, but the variance does not uniformly lag for all variables. We compare the model correlation patterns to the ones from reanalysis, one and two months earlier each, that is May $_{\text {Reanalysis }}$ to June ${ }_{C G C M}$, and so on. Regarding the size of the basin and the time scale on which the BF is active, a longer lag time between reanalysis and CGCM would not be physical. This is determined by the atmospheric and upper ocean adjustment time scales.
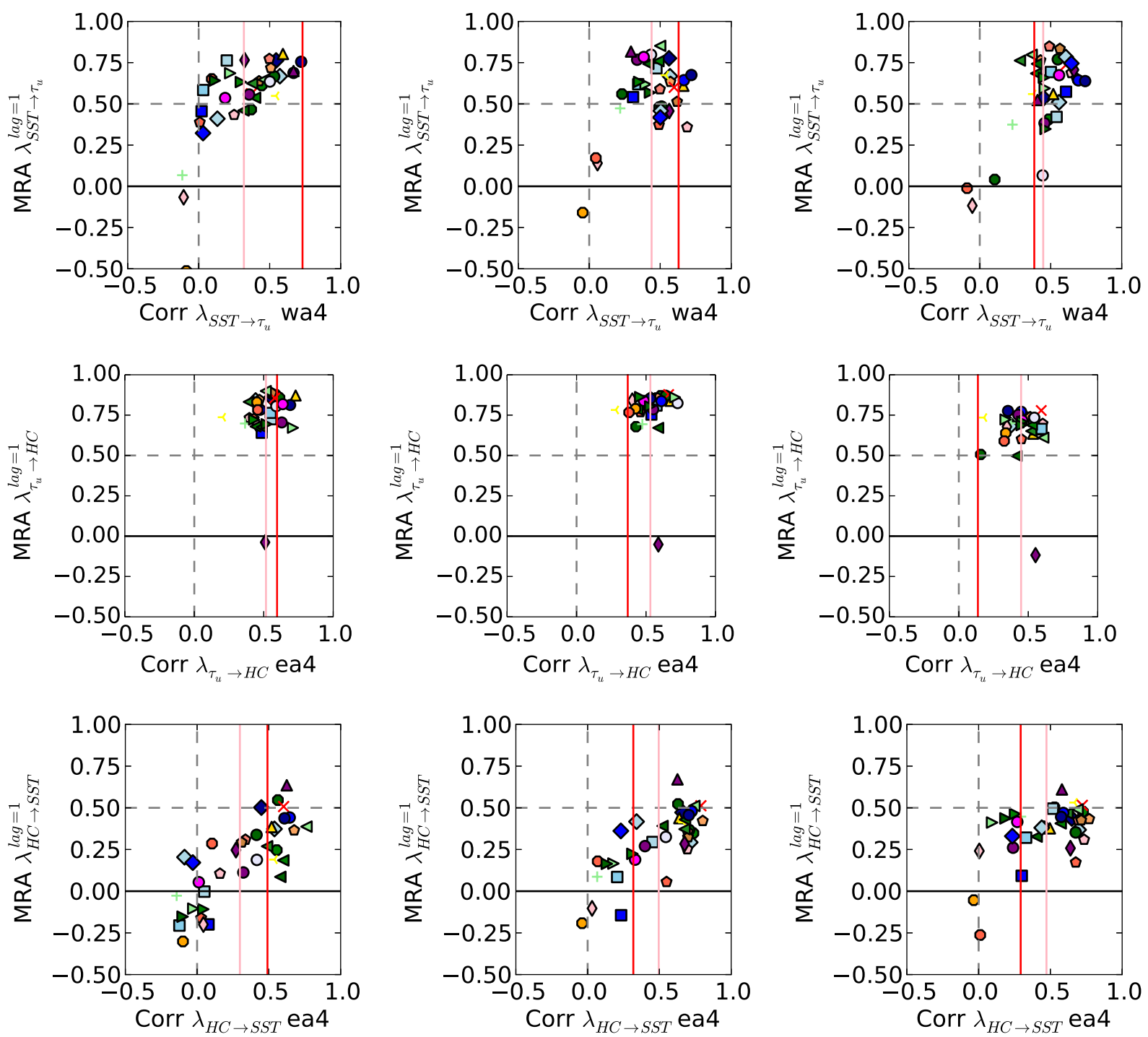

Figure 2.9: Pattern correlation vs correlation strength plot, models lagging reanalysis by one month. The three colums show the results for Mayreanalysis compared to June model $_{\text {, }}$, June $_{\text {reanalysis }}-\mathrm{July}_{\text {model }}$, and July $\mathrm{Jeanalysis}-$ August $_{\text {model }}$, respectively.

By introducing a lag of one month, the MRA is drastically improved for $\lambda_{S S T \rightarrow \tau_{u}}$ in all months (cf. Fig. 2.9). The majority of the models is now situated in the upper right corner of the plot revealing that the pattern response of $\tau_{u}$ ' to SST' is similar to the reanalysis response pattern if this lag is taken into account. In August, the CGCMs produce a 
pattern that is close to the reanalysis pattern in July. This indicates that the atmosperic response move from pure BF to another process, such as following the migration of the ITCZ, as has been suggested earlier. For $\lambda_{\tau_{u} \rightarrow H C}$ similar behaviour is observed, even though the agreement between model output and reanalysis is reasonable without a lag as well. This component of the BF is similar to the one in reanalysis in almost all of the models. The simulation of the third component of the BF, $\lambda_{H C \rightarrow S S T}$, is also improved by introducing a lag of one month between reanalysis and CGCMs, but here the model output does not reach similar agreement with the reanalysis as for $\lambda_{S S T \rightarrow \tau_{u}}$ and $\lambda_{\tau_{u} \rightarrow H C}$. For all three components of the BF a lag of two months yields similar results as a lag of one month (not shown).
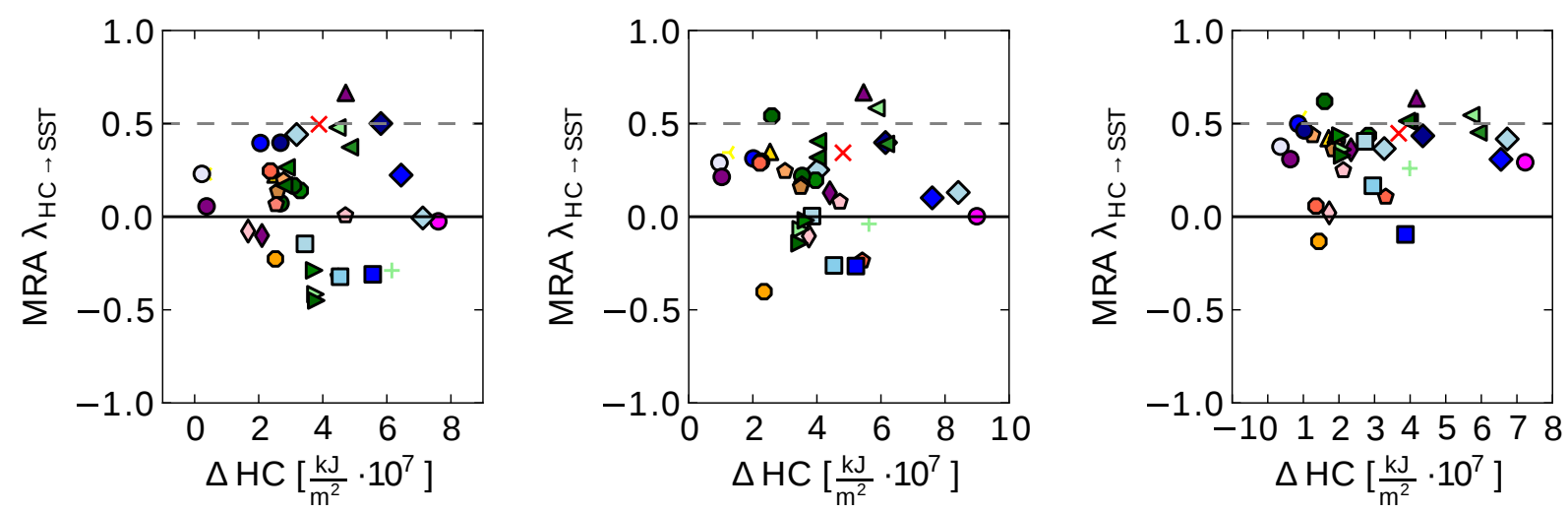

Figure 2.10: Model reanalysis agreement plotted against the absolute error of the upper ocean HC in EA4 for May, June and July. This plot shows that the mean state of the HC does not give indication for the model performance regarding $\lambda_{H C \rightarrow S S T}$.

From the results of this section we conclude that $\lambda_{S S T \rightarrow \tau_{u}}$ and $\lambda_{\tau_{u} \rightarrow H C}$ of the BF are reasonably well represented by the models, with $\lambda_{\tau_{u} \rightarrow H C}$ simulated better than $\lambda_{S S T \rightarrow \tau_{u}}$. The pattern of the correlation between HC' and overlying SST', $\lambda_{H C \rightarrow S S T}$, is not represented as well. One hypothesis is that the systematic error in the mean state of the thermocline (Richter et al., 2014b), and hence the HC, is responsible. If this is the case we expect to see a connection between the mean state error of $\mathrm{HC}$ in wa4 and the agreement between models and reanalysis $\lambda_{H C \rightarrow S S T}$ in such a way that a model which displays a reduced mean state error would score a higher MRA value. However, Fig. 2.10 reveals that no significant linear connection can be found. The misrepresentation of $\lambda_{H C \rightarrow S S T}$ can, hence, not simply be explained by the erroneous mean state (of HC) in the TA basin. Neither is there a connection between the mean state of SST in EA4 and the model performance with respect to simulating $\lambda_{H C \rightarrow S S T}$ (not shown). Note that the missing connection might indicate that the CGCMs' mean state is too far removed from the reanalysis to obtain a linear relation. 


\subsubsection{Regression Values}

Linear regression analysis (not shown) reveals that for $\lambda_{S S T \rightarrow \tau_{u}}$ and $\lambda_{\tau_{u} \rightarrow H C}$, which are reasonably well simulated in the models, there is no consistent connection between regression strength and MRA. The regression strength is under- and overestimated by the individual models, but without significant connection to the spatial pattern obtained from the correlation analysis. This is consistent with the results of the pattern correlation analysis. However, for $\lambda_{H C \rightarrow S S T}$ we notice a relationship between MRA and regression strength. This relationship becomes more obvious when the MRA value is plotted against the absolute error in regression values, as done in Fig. 2.11.
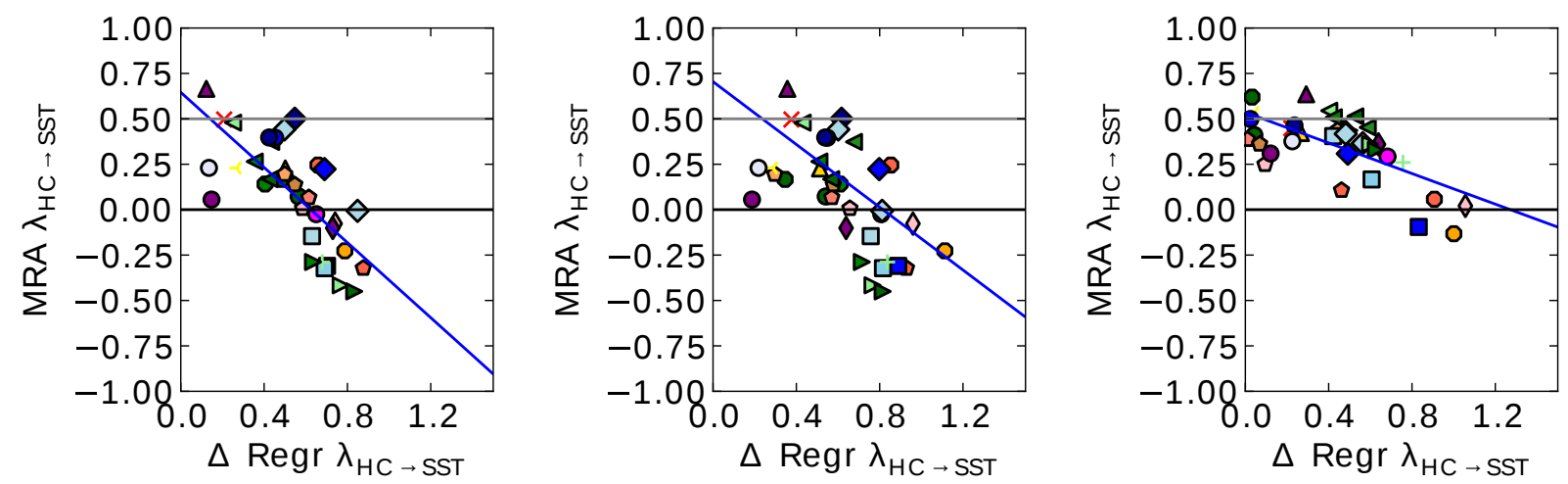

Figure 2.11: Pattern correlation plotted against absolute error regression strength for May, June, and July. The bigger the error, the smaller is the model-reanalysis agreement. Hence, the stronger the influence of HC anomaly on SST anomalies, the closer the pattern becomes to a pure BF pattern.

In general, the models underestimate the regression strength of $\lambda_{H C \rightarrow S S T}$, especially in May and June. Most models with large absolute errors in regression strength strongly underestimate the actual influence of HC' on SST'. We deduce that a model that shows a larger influence of HC' on overlying SST' is also likely to have a response pattern more similar to the actual response as obtained from reanalysis. In other words, the larger the influence of HC' on SST', the closer the $\lambda_{H C \rightarrow S S T}$ pattern is to reanalysis, i. e., the better the $\mathrm{BF}$ is simulated.

\subsubsection{Subsurface Structure}

As we have demonstrated above, the influence of HC' on overlying SST' for the individual models cannot readily be linked to a typical BF pattern in CMIP5 models. In reanalysis, on the other hand, there is a distinct pattern on the equator in May and June. When averaging the response pattern of all models we obtain a pattern (Fig. 2.12) that is similar to the one obtained from reanalysis (cf. Fig. 2.5). The correlation strength of $\lambda_{H C \rightarrow S S T}$ is, however, much lower than in reanalysis, due to different correlation patterns in the 
different CGCMs. Note that the standard deviation is large all over the basin, which indicates that individual models differ greatly in their representation of $\lambda_{H C \rightarrow S S T}$.
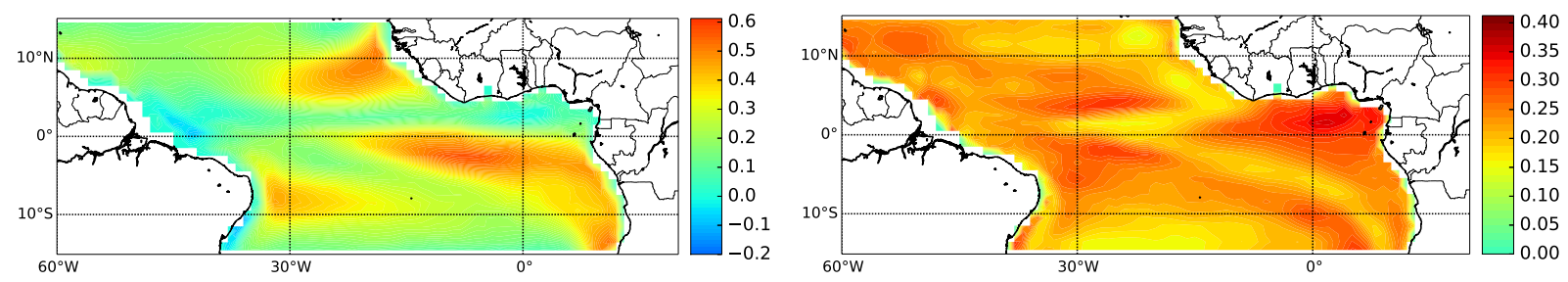

Figure 2.12: Left hand side: Model ensemble mean response pattern of $\lambda_{H C \rightarrow S S T}$. Right hand side: standard deviation of correlation values between the individual models for pattern $\lambda_{H C \rightarrow S S T}$.

We investigate the origin of the inaccurate spatial representation of $\lambda_{H C \rightarrow S S T}$ by analysing the subsurface temperatures of the equatorial Atlantic. Two temperature variance cross sections (along the equator and $30^{\circ} \mathrm{W}$ ) are shown for the reanalysis and for each of the models under investigation (Figs. 2.13, 2.14, and 2.15). In regions where the temperature variance extends to the surface, coupling between subsurface (and hence $\mathrm{HC}$ ) anomalies and SST' $^{\prime}$ is present. In the reanalysis it occurs predominantly at the equator around $10^{\circ} \mathrm{W}$ in the EA4 region (Fig. 2.13). This is vastly misrepresented by the CGCMs.
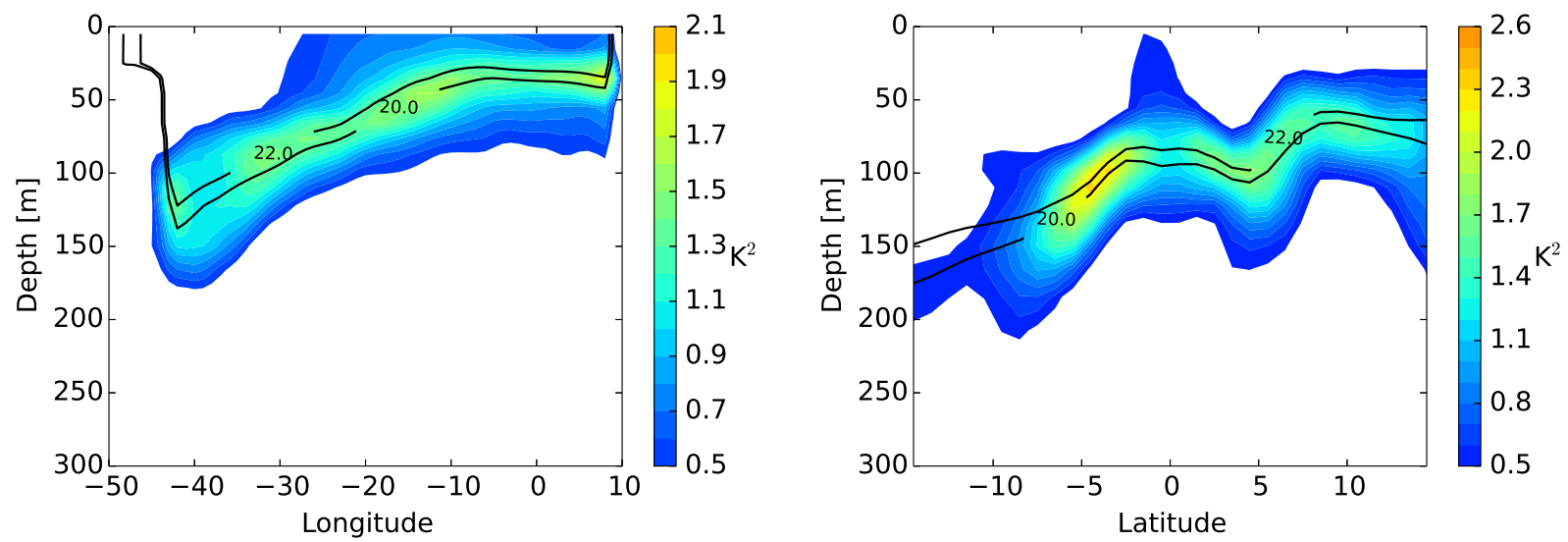

Figure 2.13: Equatorial and $30^{\circ} \mathrm{W}$ temperature variance cross sections in the month July of the series, upper $300 \mathrm{~m}$ of the TA, obtained from reanalysis.Black lines denote the $20^{\circ} \mathrm{C}$ and $22{ }^{\circ} \mathrm{C}$ isotherms.

The model output shows large deviations from the reanalysis; coupling from subsurface to surface takes place at different locations, if at all, throughout the months when the $\mathrm{BF}$ is active (only July shown). This is also illustrated by the longitude cross section in Fig. 2.15. Here, off-equatorial temperature anomalies couple with the surface much more than observed in reanalysis.

In the Pacific, Zelle et al. (2004) have identified two mechanisms through which SST' are influenced by HC', the upwelling and the wind coupling pathway. In the former, the 


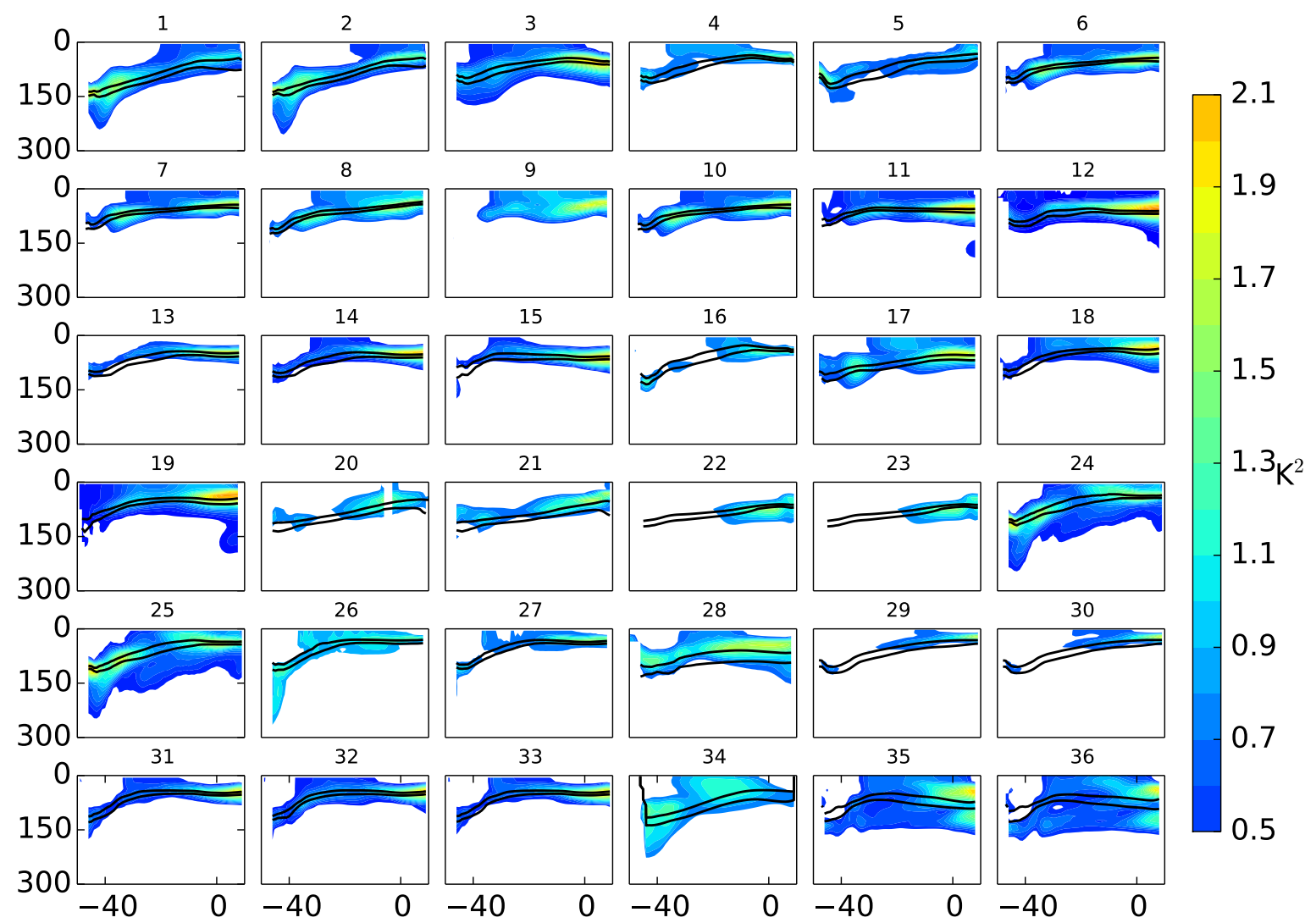

Figure 2.14: Equatorial temperature variance cross section in the upper $300 \mathrm{~m}$ of the TA for each of the models compared in this study, July. Black lines as in Fig. 2.13.

subsurface anomaly propagates vertically through mixing and upwelling. In the latter, it propagates along the equatorial thermocline, causes an SST anomaly on the eastern side of the basin (through the upwelling pathway), which causes a $\tau_{u}$ anomaly over the basin. This $\tau_{u}^{\prime}$ influences the SST' overlying the original subsurface anomaly. The wind coupling pathway is considered to be active in the western part of the Pacific, where the thermocline reaches depths of more than $120 \mathrm{~m}$. Where this pathway is active, the thermocline-SST' correlation peaks at lags of four to eight months. Considering the size of the Atlantic ocean compared to the Pacific, the lag would be considerably shorter if this pathway was active. In the reanalysis the $\lambda_{H C \rightarrow S S T}$ correlation peaks at zero month lag, and the thermocline in the eastern part of the Atlantic is very shallow (ca. $50 \mathrm{~m}$ ). This indicates that the direct pathway is dominant in the eastern part of the TA, where it forms the third component of the $\mathrm{BF}, \lambda_{H C \rightarrow S S T}$. Some models display growing correlation between HC' and SST' with increasing lag in the western part of the basin, suggesting the presence of the wind coupling pathway (not shown). However, this feature is absent in the reanalysis.

We can obtain more insight into the oceanic origin of the SST' by correlating SST's in 


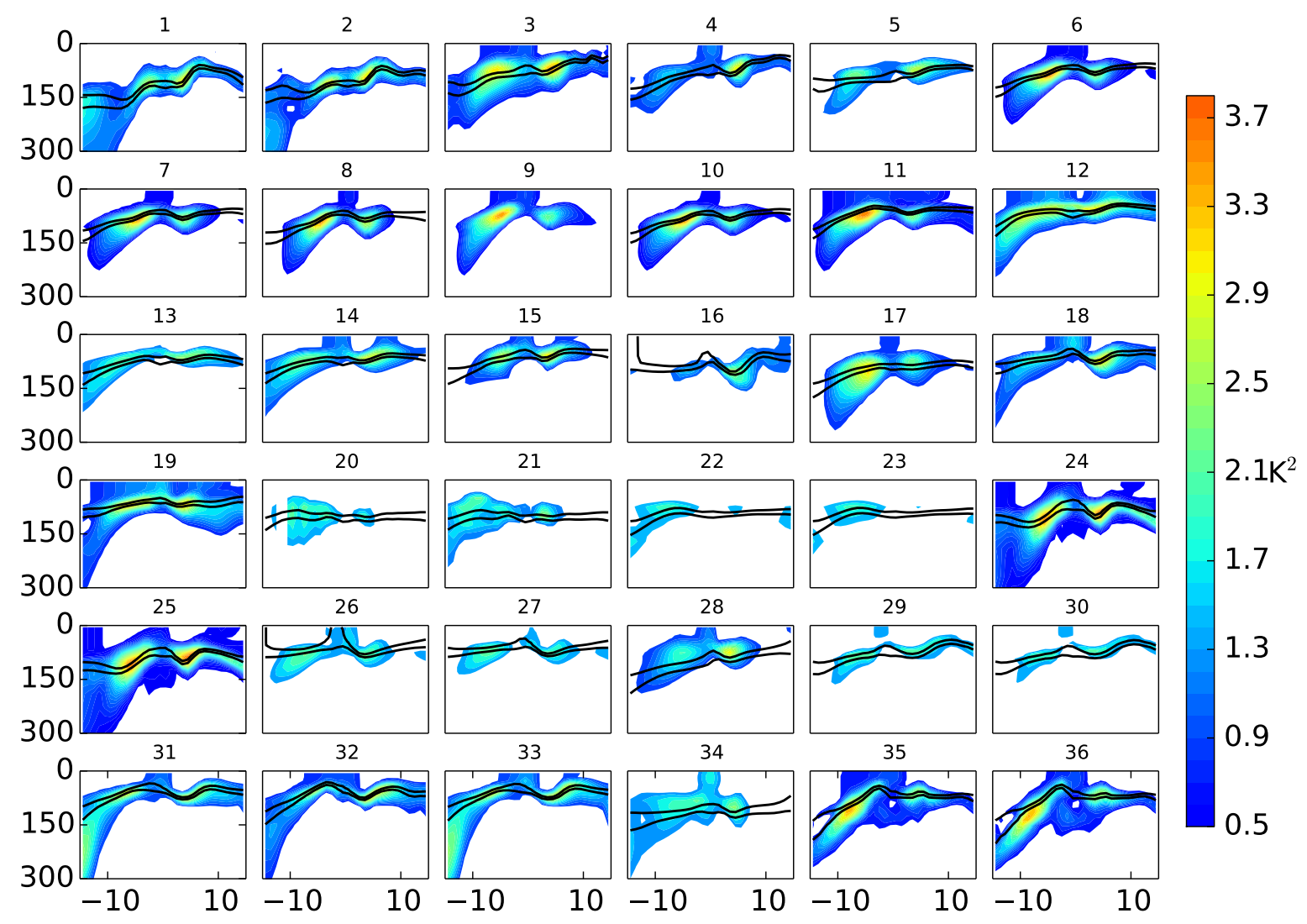

Figure 2.15: Temperature variance cross section along $30^{\circ} \mathrm{W}$ in the upper $300 \mathrm{~m}$ of the TA obtained from model output, July. Black lines as in Fig. 2.13.

the cold tongue region with subsurface temperature anomalies $\Theta_{o}$ '. In the reanalysis a clear picture of the BF and its seasonality is found (Fig. 2.16).

In February and March, SST's in the cold tongue are mostly correlated with SST' in the well mixed surface layer stretched along the equator. In April, when both $\lambda_{S S T \rightarrow \tau_{u}}$ and $\lambda_{\tau_{u} \rightarrow H C}$ become active, correlation with the underlying ocean temperature $\Theta_{o}$ becomes significant. The coupling strengthens and reaches greater depths in May and June, and retreats in July. In August it has almost disappeared. The correlation between $\Theta_{o}$ ' and SST' is significant only in the months when the BF is active. The second Niño in boral winter can also be seen. It is noteworthy though, that here the correlation between $\mathrm{SST}_{E A 4}$ ' and $\Theta_{o}$ ' is more confined to the coast, while in boreal summer it is present in the whole eastern TA. We repeated the previous analysis for each of the individual CGCMs for June and August. In June (Fig. 2.17), very few CGCMs display a $\mathrm{SST}_{E A 4}$ ' $-\Theta_{o}$ ' correlation pattern similar to the one in reanalysis. The coupling between subsurface and surface anomalies does not take place adequately.

In August (Fig. 2.18), most models simulate the coupling, although some models display spurious subsurface patterns that are not connected with the surface (e. g. panels five, 

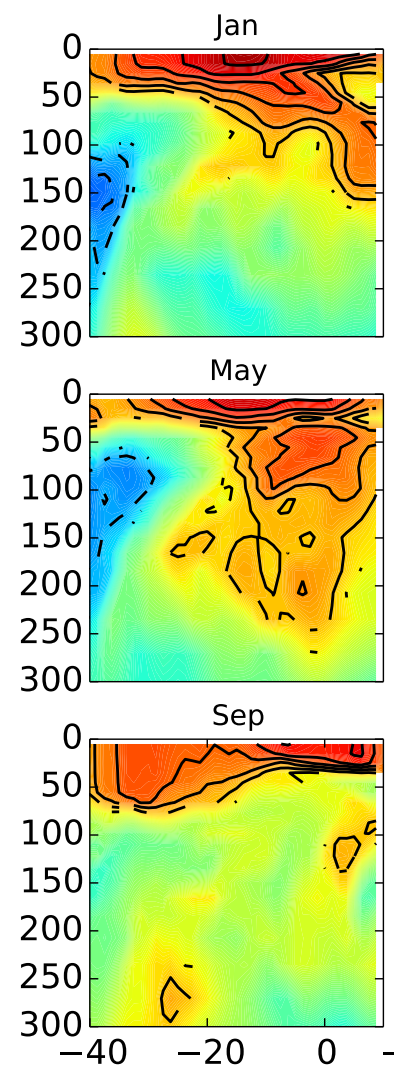

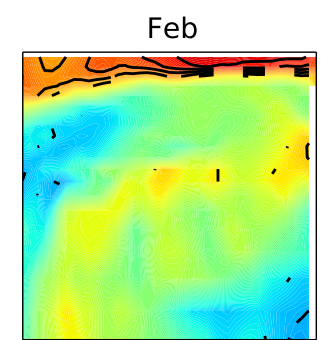

Jun

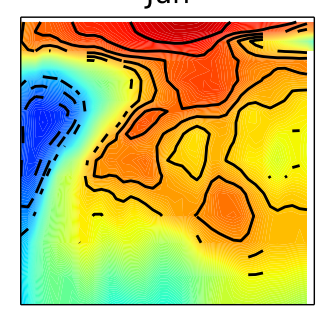

Oct

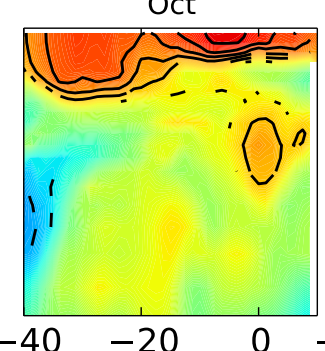

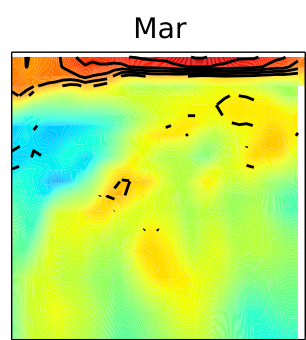

Jul

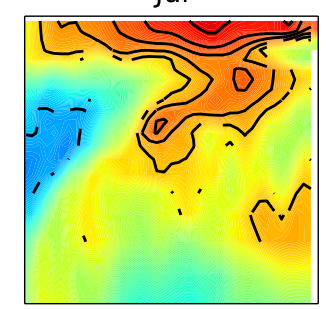

Nov

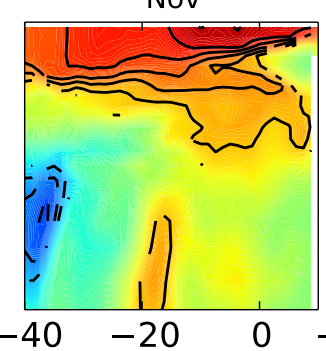

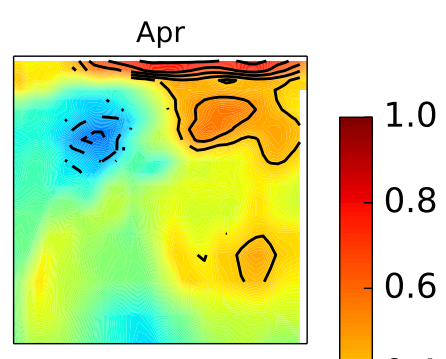

Aug

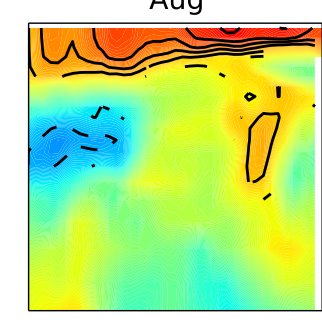

Dec

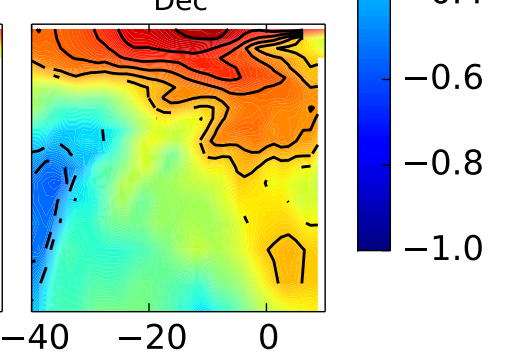

Figure 2.16: Annual cycle of equatorial $\mathrm{SST}_{E A 4}-\Theta_{o}$ correlation, reanalysis data (ORA-S4). Contours indicate statistically significant correlation at the $90 \%$ confidence level.

twelve, and fourteen). These might be associated with meridional current patterns in the CGCMs that are not present in the reanalysis. Whether this coupling is associated with the $\mathrm{BF}$ is questionable.

In August the BF loop is no longer closed. The $\mathrm{SST}_{E A 4}$ ' is not centered on the equator anymore, suggesting that other mechasnisms, such as the WES feedback, dominate. Note that we compared the correlation patterns of $\lambda_{S S T \rightarrow \tau_{u}}$ with a lag of one month. August in the CGCMs is similar to July in the reanalysis, when the pattern has departed from the equator and the BF coupling is weakening. Some models have already established an $\operatorname{SST}_{E A 4}$ ' $-\Theta_{o}$ ' correlation in July. When that is the case, the models most likely simulate the BF completely. The lack of high MRA values can then be explained by (misrepresented) off-equatorial variability. 

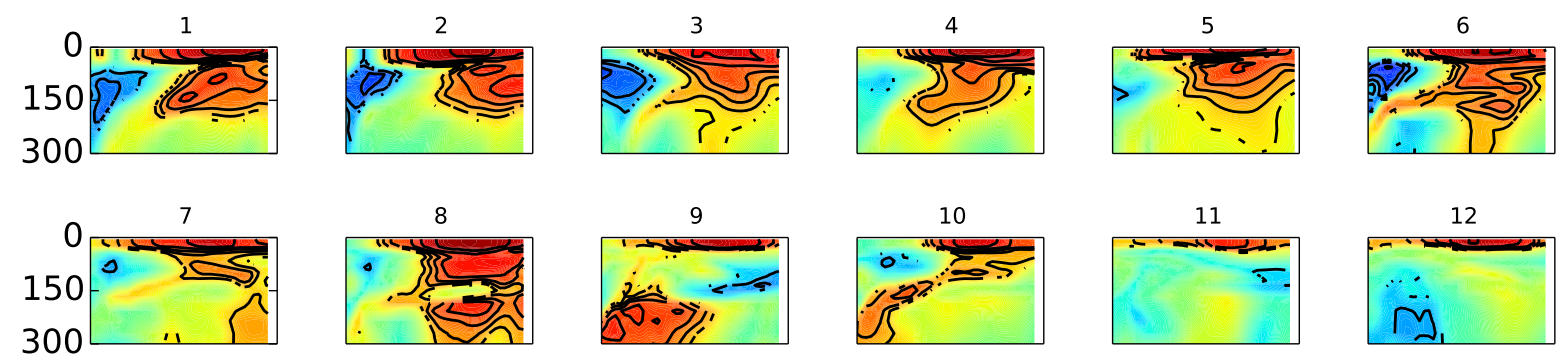

10

11
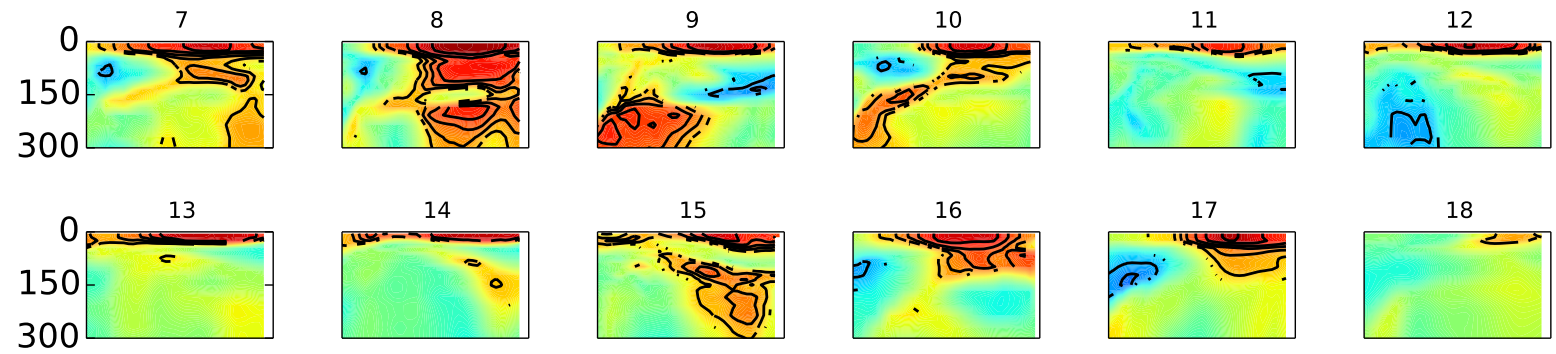

17
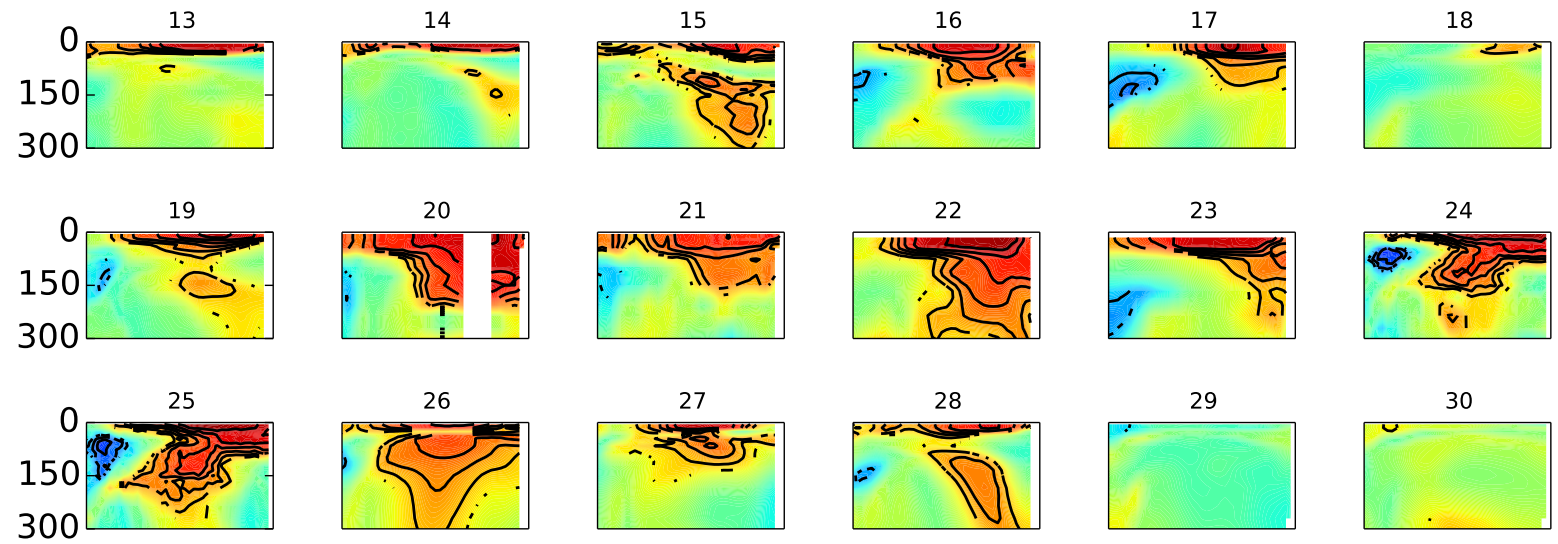

27

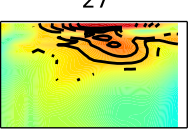

28

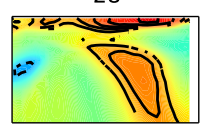

29
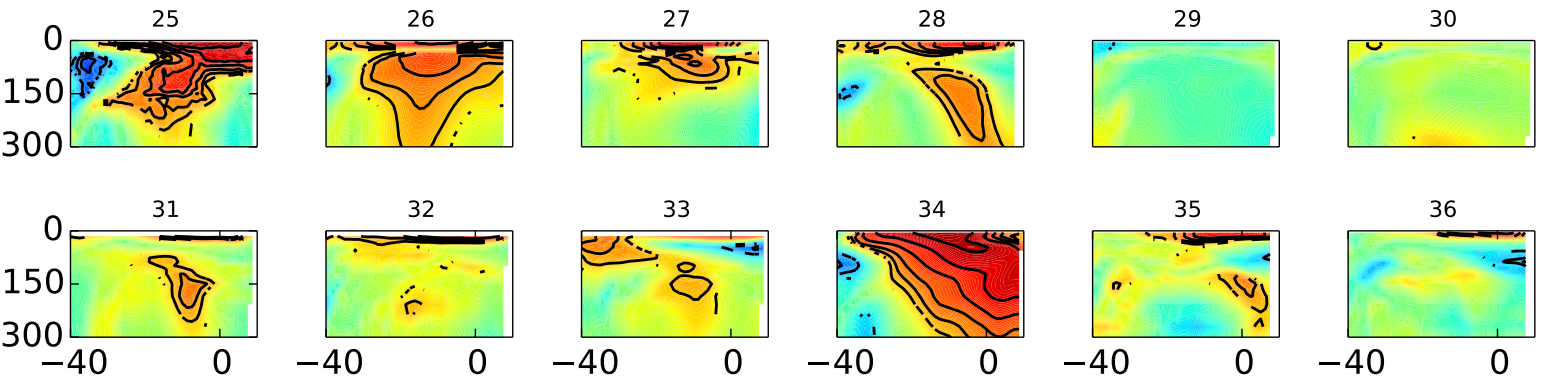

Figure 2.17: Model equatorial $\mathrm{SST}_{E A 4}-\Theta_{o}$ correlation in June. Contours as Fig. 2.16. The observerd subsurface-surface coupling is simulated by only a few models.

\subsection{Conclusion}

In this study we investigate the BF in the tropical Atlantic ocean from both reanalysis data and CMIP5 model output. The reanalysis data clearly shows the presence of a zonal mode which is driven by the BF, confirming previous studies. The monthly stratified analysis shows that the BF is not equally active throughout the year, it is strongest in May and June and begins to fade again in July. $\lambda_{S S T \rightarrow \tau_{u}}$ and $\lambda_{\tau_{u} \rightarrow H C}$ are reasonably well simulated by the models based on correlation strength and spatial pattern. While $\tau_{u}$ ' in the western part of the basin reacts to SST' $E A 4$ within a month, we introduce a lag between $\tau_{u}$ ' and HC' in order to exclude direct influences of $\tau_{u}$ ' on SST' in the eastern part of the basin. By evaluating the eastern equatorial HC' response to $\tau_{u}{ }^{\prime} W A 4$ with a lag of one month we allow for a Kelvin wave to propagate from west to east. The agreement of $\lambda_{S S T \rightarrow \tau_{u}}$ and $\lambda_{\tau_{u} \rightarrow H C}$ between reanalysis and model output improves further when introducing a lag of one month between them, correcting for the lag of the annual cycle (of variance) in the CGCMs. We conclude that the physical mechanisms behind $\lambda_{S S T \rightarrow \tau_{u}}$ and $\lambda_{\tau_{u} \rightarrow H C}$ are 

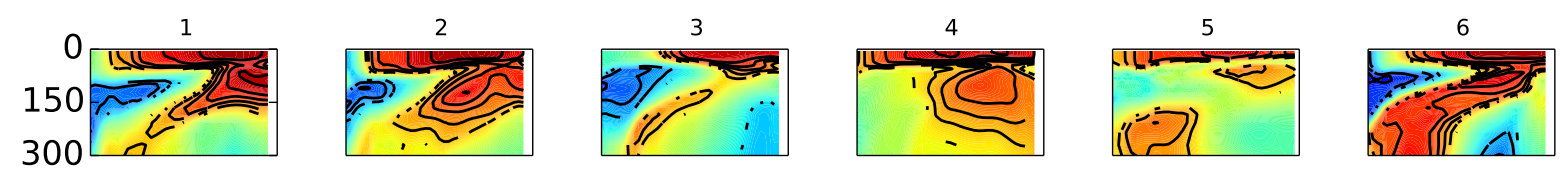
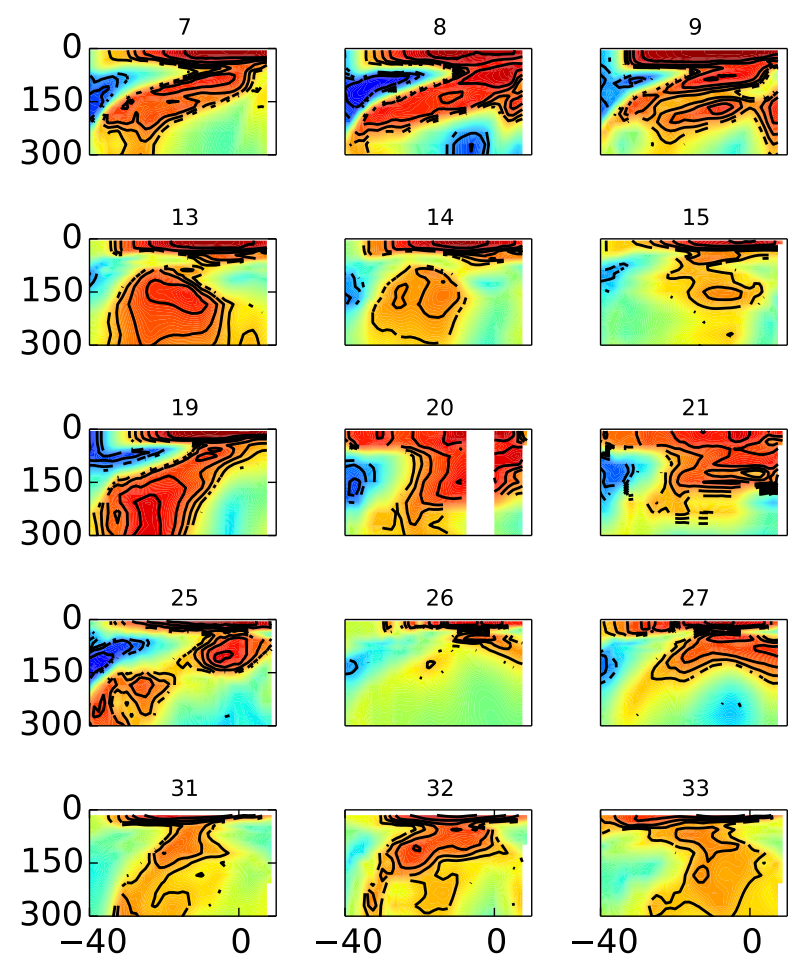

14
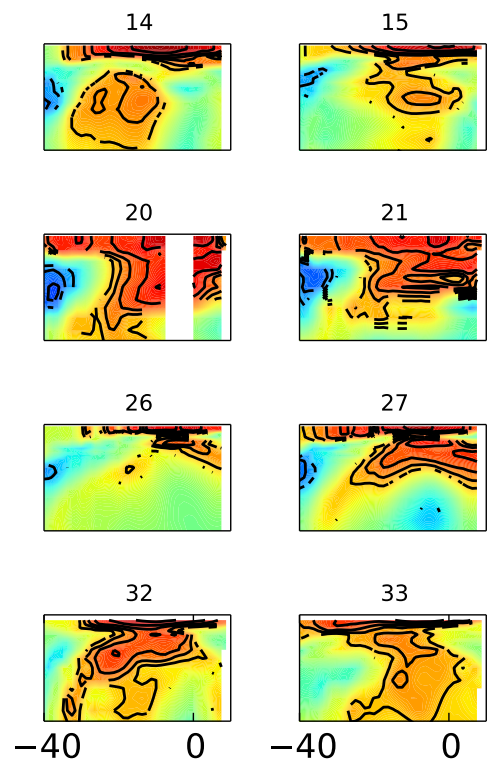

21

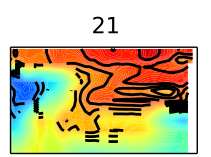

27
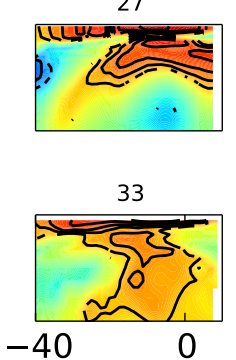

10

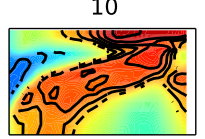

11
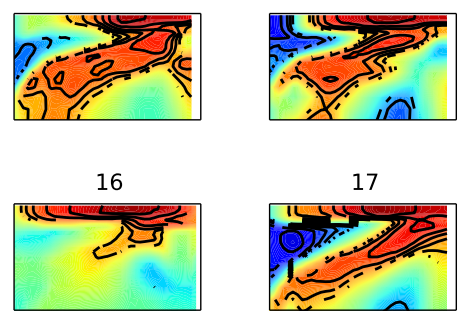

22

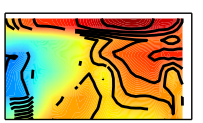

28
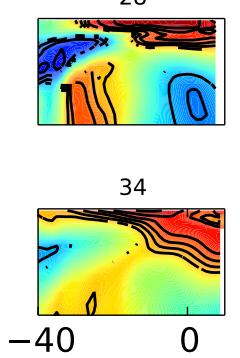

17

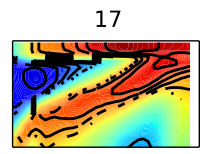

23

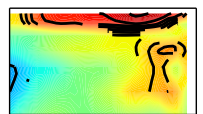

29

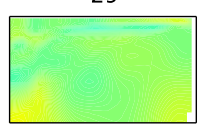

35

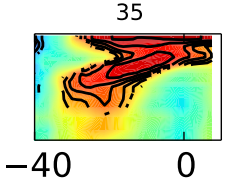

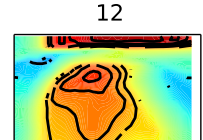

18

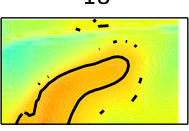

24

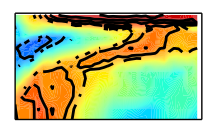

30

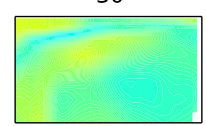

36

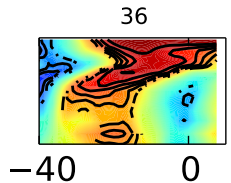

Figure 2.18: As Fig. 2.17, but for August. The CMIP5 models display an interaction between subsurface- and surface temperature later in the year.

simulated well by the models, but that the lag in the annual cycle introduces a lag in the timing of the BF. $\lambda_{H C \rightarrow S S T}$, on the other hand, is generally not well represented by the models. The patterns of the responses of CGCM output and reanalysis agree to less than $25 \%$ in almost all cases. We note that there is a significant correlation between HC' and SST' in the eastern part of the basin, but its location and pattern are significantly different from the one detected in reanalysis. No significant linear correlation between mean state error of the $\mathrm{HC}$ in the eastern part of the basin and the model-reanalysis agreement could be found, so the reason for the error in the third component of the loop could not conclusively be linked to the mean state bias of SST and HC. This does not exclude the possibility that individual models perform better when their mean state is corrected. In fact, this was recently shown to be the case for the Kiel Climate Model, which does not belong to the CGCM ensemble investigated here (Ding et al., 2015). We speculate that differences between the mean state of the CGCMs and the reanalysis mean state may be too large to display a linear relationship between the mean state error and the performance with respect to the BF. Examining the subsurface temperature variance provides insight into the origin of the largely varying $\lambda_{H C \rightarrow S S T}$ response fields. This component of the 
$\mathrm{BF}$ describes an interaction between subsurface and surface anomalies. In the equatorial cross section of temperature variance in the reanalysis there is a clear connection between subsurface and surface anomalies. Here, mixing takes place and SST is influenced by subsurface temperatures. None of the models is able to reproduce this structure. Hence, while there is some interaction indicated by the $\lambda_{H C \rightarrow S S T}$ correlation, the location of this interaction is different from reanalysis. This is also demonstrated by longitude-depth cross sections that show $S S T_{E A 4}{ }^{\prime}-\Theta_{o}$ ' correlations. The seasonality displayed by reanalysis is modelled by hardly any of the CGCMs. When the correlation decreases in reanalysis, it increases in the models and even persisits throughout the year (not shown). The timing between $\lambda_{S S T \rightarrow \tau_{u}}$ and $\lambda_{\tau_{u} \rightarrow H C}$ and the subsurface-surface coupling is misplaced in such a way that the full BF loop is most likely not closed for most of the models. $\lambda_{H C \rightarrow S S T}$ in the CGCMs becomes active too late in the year. The center of atmospheric sensitivity to $\mathrm{SST}_{E A 4}$ ' has moved north of the equator, indicating that other processes play a more important role than the $\mathrm{BF}$ along the equator. The lack of seasonality displayed in $S S T_{E A 4}$ ' $-\Theta_{o}$ ' in the CGCMs hints on other processes inducing the $S S T_{E A 4}$ '- $\Theta_{o}$ ' coupling, rather than being initiated by the $\mathrm{BF}$.

Based on the results of this study, we conclude that the erroneous vertical oceanic stratification influences the interannual variability in the region by hindering $\lambda_{H C \rightarrow S S T}$ from being represented correctly. Even though the models display an SST annual cycle much weaker than in reanalysis, $\lambda_{S S T \rightarrow \tau_{u}}$ and $\lambda_{\tau_{u} \rightarrow H C}$ are reasonably well simulated, albeit with an error in timing because of the lags in seasonality. This disproves our hypothesis that the functioning of the BF depends on the correctly simulated annual cycle. The mean state of the TA is misrepresented in the CGCMs, but the influence of perturbations of variable $a\left(\mathrm{SST}, \tau_{u}\right)$ on variable $b\left(\tau_{u}, \mathrm{HC}\right)$ is still present. This is in line with earlier findings that even though the mean state of the TA is heavily biased, interannual variability can still be reasonably represented (Richter et al., 2014b). However, biases in the subsurface temperature variance affect the spatial pattern of $\lambda_{H C \rightarrow S S T} . \lambda_{H C \rightarrow S S T}$ explains a large part of SST variability in the region, and exactly this part of the feedback is not simulated well. The errors in the simulated subsurface thermal structure can be due to both ocean mixing and/or arise from shortcomings of the atmospheric forcing which sets the structure of the ventilated thermocline. This should be the focus of further investigation and a starting point for work on model improvement in order to improve the $\mathrm{HC}$ SST relationship in the eastern tropical Atlantic. 


\section{Chapter 3}

\section{The South-Eastern Tropical Atlantic SST bias investigated with a coupled atmosphere-ocean single column model at a PIRATA mooring site.}

This chapter is based on:

Deppenmeier, A.-L., Haarsma, R. J., Heerwaarden, C. v., and Hazeleger, W.: The South-Eastern Tropical Atlantic SST bias investigated with a coupled atmosphere-ocean single column model at a PIRATA mooring site., under review at Journal of Climate 
The South-Eastern Tropical Atlantic SST bias investigated with a coupled 44 atmosphere-ocean single column model at a PIRATA mooring site.

\section{Abstract}

Warm sea surface temperature biases (SST) in the tropical Atlantic form a longstanding problem in coupled general circulation models (CGCMs). Considerable efforts to understand the origins of these biases and alleviate them have been undertaken, but state-of-the-art CGCMs still suffer from biases very similar to those of the generation of models before. In this study, we use a powerful combination of in-situ moored buoy observations and a new coupled ocean-atmosphere single column model (SCM) with identical parameterisation as a 3D CGCM to investigate the SST bias. We place the SCM at the location of a PIRATA mooring in the south-eastern tropical Atlantic, where large SST biases occur in CGCMs.

The SCM version of the state-of-the-art coupled GCM EC-Earth performs well for the first five days of the simulation. Then, it develops an SST bias very similar to that of its 3D counterpart. Through a series of sensitivity experiments we demonstrate that the SST bias can be reduced by $70 \%$. We achieve this result by enhancing the turbulent vertical ocean mixing efficiency in the ocean parameterisation scheme. The under-representation of vertical mixing in 3D GCMs is a candidate for causing the warm SST bias.

We further show that surface shortwave radiation does not cause the SST bias at the location of the PIRATA mooring. Rather, a warm atmospheric near-surface temperature bias and a wet moisture bias contribute to it. Strongly nudging the atmosphere to profiles from reanalysis data reduces the SST bias by $40 \%$. 


\subsection{Introduction}

Tropical Atlantic sea surface temperatures (SST) display large variability on inter-annual timescales, and a strong seasonal cycle. State of the art coupled global climate models (GCMs) struggle to capture the cooling in the south-eastern tropical Atlantic, as a result of which they suffer from large warm SST biases in that region (Richter and Xie, 2008; Richter et al., 2012; Wang et al., 2014). These biases hamper efforts to reliably predict societal relevant climate events (Stockdale et al., 2006), such as the West African Monsoon and the Atlantic Niño.

In boreal summer, the south-eastern tropical Atlantic cools strongly and rapidly. Simultaneously, a cold tongue forms on the equatorial eastern Atlantic, extending as far as $20^{\circ} \mathrm{W}$ (Fig. 3.1a, visible in June, July, and August). In boreal fall, the cold tongue recedes and the cold waters in the south east warm gradually.

On the south-eastern edge of the cold tongue $\left(6^{\circ} \mathrm{S}, 8^{\circ} \mathrm{E}\right)$, located in the region of strong annual cooling (Fig. 3.1b), the Pilot Research Moored Array in the tropical Atlantic (PIRATA (Servain et al., 1998)) offers observational data to fill the gap of our knowledge of the ocean and air-sea interaction processes in this region. At the location of this buoy, SST cool by several degrees during boreal summer (Fig. 3.1b). The cooling in 3D GCMs is much weaker, as indicated at the example of EC-Earth, also in Fig. 3.1b. The insufficient cooling leads to the large typical positive sea surface temperature biases in the region. During the first five months of the year, the 3D model accurately captures the sea surface temperature in the south eastern tropical Atlantic. With the onset of the strong cooling the bias develops, it is first sizeable in June. This makes June the ideal month to study the bias, as it is the month in which it establishes.

Recently, the seasonal heat budget at this site has been analysed by (Scannell and McPhaden, 2018) for the five years in which daily data record is available. The authors find that in boreal summer reduced shortwave forcing and vertical turbulent entrainment into the upper ocean mixed layer are the main causes for the sea surface temperature cooling. The latter process occurs at scales too small to be explicitly captured in the ocean component of 3D models, and has to be added via parameterisation. The specifics of the parameterisation determine the strength of the mixing included in the model. The underrepresentation of this vital process is a strong candidate for producing the warm bias (Hazeleger and Haarsma, 2005; Exarchou et al., 2017; Planton et al., 2018).

Other origins of the warm bias have been suggested to arise in the atmosphere, for example, from excessive shortwave radiation (Huang et al., 2007; Hu et al., 2008), or insufficient wind forcing (Richter et al., 2012; Voldoire et al., 2014; Koseki et al., 2018), or from an atmospheric moisture bias (Hourdin et al., 2015). A recent multi-model study highlights the role of wind stress forcing in the bias development (Voldoire et al., 2019), but also 
The South-Eastern Tropical Atlantic SST bias investigated with a coupled 46 atmosphere-ocean single column model at a PIRATA mooring site.

Figure 3.1: Seasonal cycle of surface temperatures in the tropical Atlantic from ERA-Interim reanalysis data (Dee et al., 2011) (1979-2013) (panel a). The star marks the location of the buoy we use in this study, at $8^{\circ} \mathrm{E}, 6^{\circ} \mathrm{S}$. It is part of the PIRATA array (Servain et al., 1998). Panel b shows the seasonal cycle of sea surface temperature from buoy data, averaged from daily data over the years 2014-2018, and for the coupled global version of EC-Earth.
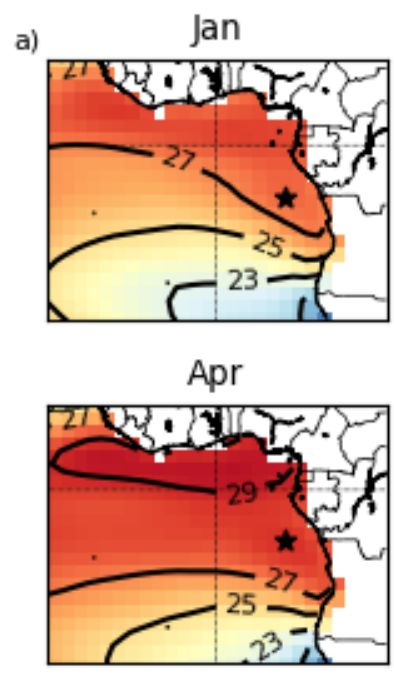

Jul

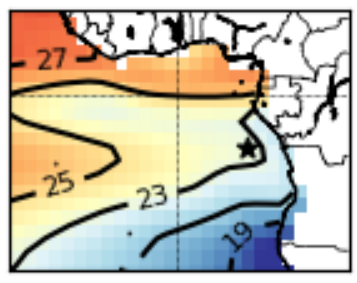

Oct

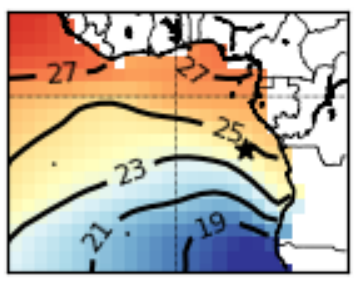

b)

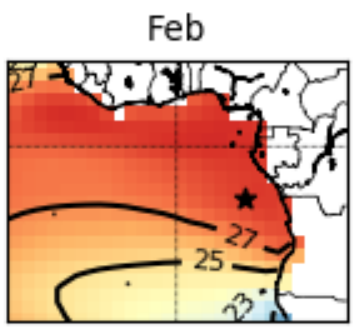

May

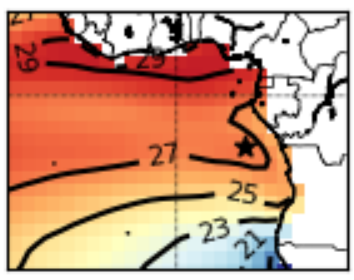

Aug

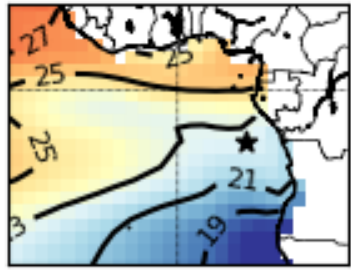

Nov

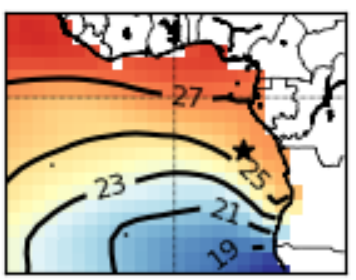

Mar

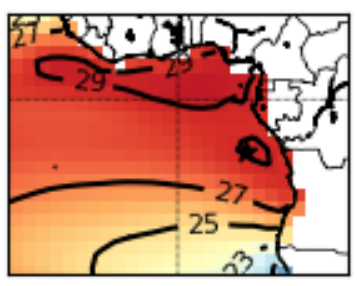

Jun

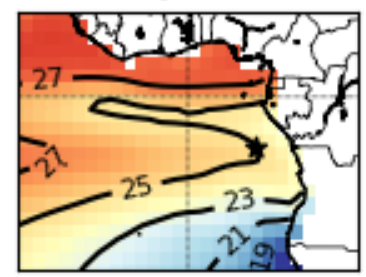

Sep

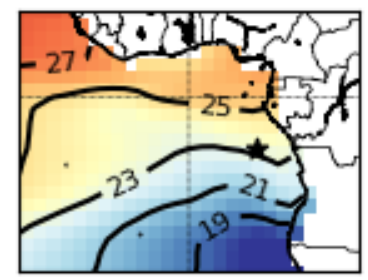

Dec

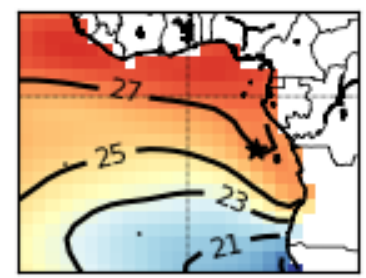

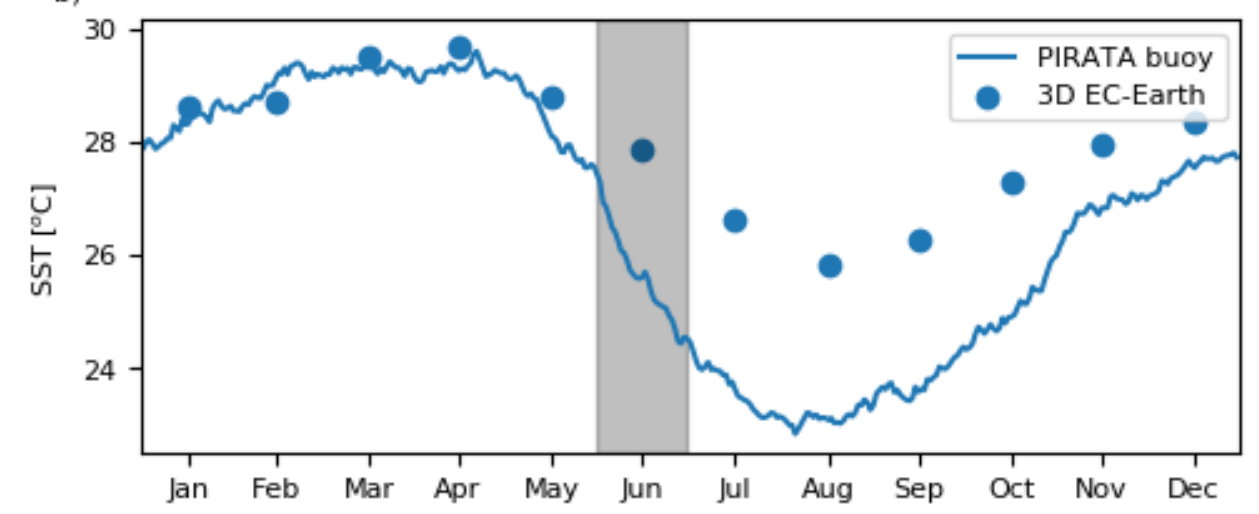


shows that it cannot explain the entire bias and sometimes even has limited effect (as is the case for EC-Earth, which we use here). Other studies have highlighted the contribution of the ocean model (Xu et al., 2014b), its horizontal and vertical resolution (Seo et al., 2006; Doi et al., 2012; Small et al., 2014), advection (Goubanova et al., 2019) and turbulent processes (Hazeleger and Haarsma, 2005; Exarchou et al., 2017; Planton et al., 2018) to the bias formation. The question of the south-eastern tropical Atlantic warm bias is not yet resolved and more analysis is clearly necessary to trace its origins.

In this study, we use an ocean-atmosphere coupled single column version of the coupled GCM EC-Earth (Hazeleger et al., 2010) to investigate the bias formation in the southeastern tropical Atlantic, at the location of the $6^{\circ} \mathrm{S}, 8^{\circ} \mathrm{E}$ PIRATA mooring. With the single column model (SCM) we can investigate processes active on very short time scales. This is impractical, if not impossible, with the three dimensional model. With the coupled $\mathrm{SCM}$, as opposed to the standalone version of the atmosphere and the ocean, we can investigate coupled air-sea processes, and the effect of the model bias in one component on the other component. In this work we first test the impact of the atmosphere on the ocean, and then focus on ocean parameterisation.

The short runtime of the SCM allows us to perform a range of sensitivity experiments and explore the parameter space that determines the short timescale processes of our interest. By choosing a location for which in-situ data are available we are able to closeley compare and evaluate the model performance. Additionally, we can employ observed data to force the model.

The paper is structured as follows. We describe the model in Section 3.2. In Section 4.2 we describe the data used in this study, and the set up of the SCM experiments. In section 3.4.1 we evaluate the single column model performance, before moving on to atmospheric sensitivity experiments in section 3.4.2 and ocean experiments in section 3.4.3. The results are summarised and discussed in section 3.5.

\subsection{Model description}

We use a novel coupled ocean-atmosphere single column model (SCM, Hartung et al. (2018)) derived from the 3D host model EC-Earth3 (after Hazeleger et al. (2010, 2012)).

The SCM consists of the NEMO ocean model version 3.6 (Madec et al., 2011), which includes the sea ice model LIM3 (Vancoppenolle et al., 2008), and the Open Integrated Forecasting System OpenIFS cycle 40r1 (https://confluence.ecmwf.int/display/OIFS/About+OpenIFS) for the atmosphere, with the land surface model H-Tessel (Balsamo et al., 2009). Coupling between 


\section{The South-Eastern Tropical Atlantic SST bias investigated with a coupled 48 atmosphere-ocean single column model at a PIRATA mooring site.}

the ocean and atmosphere is handled by OASIS3-MCT (Valcke, 2013), similar to the way the components couple in three dimensional EC-Earth.

OpenIFS solves the one dimensional primitive equations for momentum (Eqs. 3.1 and 3.2), thermodynamics (Eq. 3.3), and moisture (Eq. 3.4) for the atmosphere:

$$
\begin{aligned}
& \frac{\partial u}{\partial t}=-\dot{\eta} \frac{\partial u}{\partial \eta}+F_{u}+f\left(v-v_{g}\right)+P_{u}+\frac{u_{r}-u}{\tau_{a}} \\
& \frac{\partial v}{\partial t}=-\dot{\eta} \frac{\partial v}{\partial \eta}+F_{v}-f\left(u-u_{g}\right)+P_{v}+\frac{v_{r}-v}{\tau_{a}} \\
& \frac{\partial T}{\partial t}=-\dot{\eta} \frac{\partial T}{\partial \eta}+F_{T}+\frac{R T \omega}{c_{p} p}+P_{T}+\frac{T_{r}-T}{\tau_{a}} \\
& \frac{\partial q}{\partial t}=-\dot{\eta} \frac{\partial q}{\partial \eta}+F_{q}+P_{q}+\frac{q_{r}-q}{\tau_{a}}
\end{aligned}
$$

The vertical coordinate $\eta$ merges orography with pressure coordinates in the free atmosphere. $\dot{\eta}$ is the vertical velocity in this coordinate, and $\omega$ the vertical velocity in pressure coordinates. $u$ and $v$ are the horizontal velocity components, with their geostrophic contributions $u_{g}$ and $v_{g} . f$ is the Coriolis parameter, $R$ the universal gas constant and $c_{p}$ the heat capacity (both for moist air). $p$ is pressure. The terms $F_{i}$ are horizontal advection of momentum, temperature and moisture, and $P_{i}$ are parameterisations of sub-grid scale processes. The parametrised processes include radiative transfer, convection, and clouds, with its own prognostic equations for cloud liquid and ice, rain and snow water content and cloud cover. These parameterisations have been the subject of intensive research, and are not the focus of this study. Profiles can be nudged to reference states for $u_{r}, v_{r}, T_{r}$, and $q_{r}$ with a timescale $\tau_{a}$.

The surface energy budget is

$$
\begin{array}{r}
\left(1-\alpha_{i}\right)\left(1-f_{R_{s}, i}\right) R_{s}+R_{T}-\epsilon \sigma T_{s k, i}^{4}+S H_{i}+L H_{i} \\
=Q_{T}=\Lambda_{s k, i}\left(T_{s k, i}-T_{1}\right) .
\end{array}
$$

The subscript $i$ indicates that the surface grid box is subdivided into tiles, and hence a single gridbox can consist of partly ocean and partly sea ice (or land surface). The shortwave radiation at the surface $R_{s}$ is absorbed with fraction $f_{R s, i}$ and reflected with albedo $\alpha_{i}$. $R_{T}$ is downward longwave radiation, $\epsilon$ the surface emissivity, and $\sigma$ the StefanBoltzmann constant. $Q_{T}$ is the total surface heat flux, and $T_{s k, i}$ and $\Lambda_{s k, i}$ are tiled skin layer temperature and conductivity, respectively. $T_{1}$ is the upper ocean (or sea ice) layer temperature. In our case there are ocean tiles only.

The one dimensional ocean model is based on the hydrostatic equation, temperature $(T)$ and salt $(S)$ conservation (Eqs. 3.8 and 3.9), the momentum equations (Eqs. 3.6 and 3.7), and the equation of state $\rho=\rho(T, S, p)$ (polyEOS80-bsq Fofonoff and Millard Jr 
$(1983))$.

$$
\begin{aligned}
\frac{\partial u}{\partial t} & =-\frac{\partial}{\partial z} A_{v m} \frac{\partial u}{\partial z}+f v \\
\frac{\partial v}{\partial t} & =-\frac{\partial}{\partial z} A_{v m} \frac{\partial v}{\partial z}-f u \\
\frac{\partial T}{\partial t} & =-\frac{\partial}{\partial z} A_{v t} \frac{\partial T}{\partial z}+\frac{1}{\rho_{o} c_{p}} \frac{\partial I\left(F_{s o l}, z\right)}{\partial z}+Q_{T} \\
\frac{\partial S}{\partial t} & =-\frac{\partial}{\partial z} A_{v t} \frac{\partial S}{\partial z}+E-P
\end{aligned}
$$

$f$ is the Coriolis parameter as above, $\rho_{o}$ is the ocean reference density $1035 \frac{\mathrm{kg}}{\mathrm{m}^{3}}, u$ and $v$ are the horizontal momentum components. The first terms on the right hand side of Equations 3.6-3.9 describe the effect of turbulent mixing on the ocean column. $A_{v m}$ and $A_{v t}$ are the vertical turbulent viscosity and diffusivity coefficients, respectively. The coefficients have to be determined via a turbulence closure paramterisation scheme, which is described below. In the one dimensional model, vertical turbulent mixing is the only parametrised process. $I\left(F_{\text {sol, }, z}\right)$ is the penetrative part of the surface solar radiation, and $E-P$ is the fresh water flux at the ocean surface due to evaporation and precipitation. Nudging to reference profiles is, at the moment, not implemented in the model. Scannell and McPhaden (2018) find horizontal advection to play only a very minor role in the heat budget, which justifies the use of the 1D model without applying large scale forcing at this location.

At the coupling interface between the atmosphere and the ocean, the ocean receives wind stress, turbulent, and radiative surface fluxes (split into solar and non-solar), and the fresh water budget from the atmosphere. This impacts the boundary conditions of the ocean according to the following equations, where $z$ is the depth of the column, and $\tau_{u}$ and $\tau_{v}$ are the horizontal wind stress components.

$$
\begin{aligned}
A_{v m} \frac{\partial u}{\partial z} & =\frac{\tau_{u}}{\rho_{0}} \\
A_{v m} \frac{\partial v}{\partial z} & =\frac{\tau_{v}}{\rho_{0}} \\
A_{v t} \frac{\partial T}{\partial z} & =\frac{Q_{t}}{\rho_{0} c_{p}} \\
A_{v t} \frac{\partial S}{\partial z} & =\frac{(E-P) S_{0}}{\rho_{0}}
\end{aligned}
$$

The setup of the single column model and the processes relavant to this study are schematically shown in Fig. 3.2. 

atmosphere-ocean single column model at a PIRATA mooring site.

Figure 3.2: Schematic of the coupled ocean atmosphere single column version of EC-Earth used in this study. We force the atmosphere with temperature, moisture, and momentum advection. At the interface between the atmosphere and the ocean the model components exchange fluxes, among which the turbulent (latent and sensible heatflux, LH+SH) and radiative heat fluxes (shortwave (SW) and longwave (LW)) indicated with arrows. The ocean further receives momentum forcing from the atmosphere $(\tau)$, and mass fluxes (not indicated). In the ocean column, vertical mixing is the only parameterised process solved for with the turbulent kinetic energy scheme (Mix TKE).

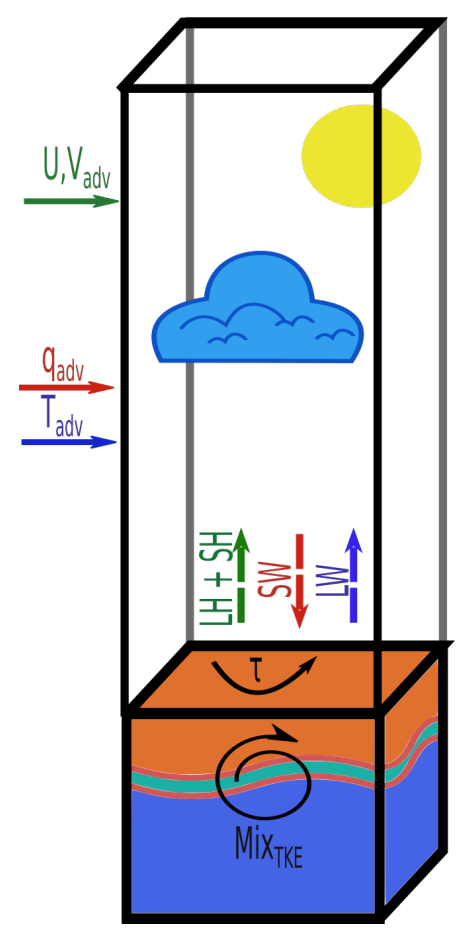

\subsubsection{Turbulent Vertical Mixing in the Ocean}

As mentioned above, the sub-grid scale paramterisation in the single column model consists solely of turbulent vertical mixing. It is based on a turbulent kinetic energy (TKE) closure scheme (Blanke and Delecluse, 1993; Gaspar et al., 1990; Madec et al., 2011), which solves for the turbulent coefficients $A_{v t}$ and $A_{v m}$ with the prognostic TKE equation:

$$
\begin{array}{r}
\frac{\partial \bar{e}}{\partial t}=\frac{C_{W I} \cdot|\tau|}{\rho_{0}}+\frac{w_{L C}{ }^{3}}{H_{L C}}+A_{v m}\left[\left(\frac{\partial u}{\partial z}\right)^{2}+\left(\frac{\partial v}{\partial z}\right)^{2}\right]-A_{v t} \cdot N^{2} \\
+\frac{\partial}{\partial z}\left[A_{v m} \frac{\partial \bar{e}}{\partial z}\right]-C_{\epsilon} \frac{\bar{e}^{\frac{3}{2}}}{l_{\text {diss }}}+C_{W F} \cdot \bar{e} \cdot \exp ^{-z}
\end{array}
$$

The change of available turbulent kinetic energy $\bar{e}$ in time is the sum of the following contributions to the TKE budget, in the order of appearance on the right hand side: production by wind input at the surface, Langmuir cell contributions, production by shear, destruction by stratification, vertical diffusion, Kolmogorov dissipation, and internal and 
surface wave breaking. In Equation 3.14, $C_{W I}$ is a parameter for the wind input, $|\tau|$ is the wind stress, $\omega_{L C}$ is the Langmuir circulation velocity, and $H_{L C}$ the depth of the Langmuir cell. The Langmuir circulation strength is calculated according to

$$
w_{L C}=C_{L C} \cdot u_{s} \cdot \sin \left(\frac{\pi z}{H_{L C}}\right)
$$

with $u_{s}=0.377 \cdot \sqrt{|\tau|}, H_{L C}$ dependent on the column stability given by $N^{2}$, and $C_{L C}$ a coefficient influencing the circulation strength.

Furthermore, $N^{2}$ is the Brunt-Väisälä frequency, $C_{\epsilon}$ and $l_{\text {diss }}$ are the dissipation coefficient and length scale. The latter is calculated according to

$$
l_{\text {diss }}=\sqrt{\frac{2 \bar{e}}{N^{2}}},
$$

and is furthermore bound by phyical considerations (e.g., the length close to the surface cannot be larger than the distance to the surface). $C_{W F}$ is the wave breaking coefficient indicating the fraction of energy that penetrates below the mixed layer.

The turbulent coefficients $A_{v m}$ and $A_{v t}$, vertical eddy viscosity and diffusivity, are calculated according to Eqs. 3.17 and 3.18:

$$
\begin{aligned}
A_{v m} & =C_{d i f f} \cdot l_{\text {mix }} \cdot \sqrt{\bar{e}} \\
A_{v t} & =A_{v m} / P_{r t}
\end{aligned}
$$

Here, $C_{d i f f}$ is a coefficient for which the numerical value has to be derived from observations, it is related to the vertical eddy mixing efficiency (Gaspar et al., 1990). $l_{\text {mix }}$ is the mixing length across which the turbulence can act, equal to $l_{\text {diss }}$. The Prandtl Number $P_{r t}$ in Eq. 3.18 is dependent on the Richardson Number $R i=\frac{N^{2}}{\left(\frac{\partial U}{\partial z}\right)^{2}}$, but in fact is equal to 1 in all cases considered. Hence, $A_{v m}$ and $A_{v t}$ have the same value.

Of the coefficients $C_{i}$, some are more certain than others. $C_{\epsilon}$, for example, is generally agreed to take on the value 0.7 (Gaspar et al., 1990). Similarly, $C_{W I}$ (wind input coefficient) and $C_{W F}$ (wave fraction penetration below the mixed layer) are chosen to represent the average impact of medium aged waves. The Langmuir Coefficient, on the other hand, is set to 0.15 as a default, but can assume values up to 0.45 (Axell, 2002). $C_{\text {diff }}$ can be estimated according to:

$$
C_{d i f f}=\frac{1}{2} \cdot \gamma \cdot P_{d l} * C_{\epsilon},
$$

where $C_{\epsilon}=0.7$ and $P_{d l}=1$. For the ocean vertical mixing efficieny $\gamma$, observational estimates exist (see Gaspar et al. (1990) and references therein). From these measurements it results that $C_{\text {diff }}$ can assume values between 0.035 and 0.28 . The default value in NEMO, $C_{d i f f}=0.1$, is on the lower end of the possible values for $C_{d i f f}$. 


\section{The South-Eastern Tropical Atlantic SST bias investigated with a coupled 52 atmosphere-ocean single column model at a PIRATA mooring site.}

\subsection{Experimental Setup}

The sea surface temperature bias in coupled global climate models typically peaks in boreal summer, coinciding with the period of rapid observed cooling. We first examine whether the single column model displays similar behaviour, focusing on June, when the observed cooling is strongest and the bias begins to develop. We perform an ensemble of five simulations for the years in which there are high temporal resolution buoy observations available (2014-2018).

To account for large scale circulation impacts, the SCM is forced with horizontal wind, temperature and moisture advection. This forcing is extracted from 3-hourly ERA-Interim data (Dee et al., 2011) from the grid point closest to the buoy. The grid point is approximately $50 \mathrm{~km}$ away $\left(5.96^{\circ} \mathrm{S}, 8.44^{\circ} \mathrm{E}\right)$. We assume that the large scale circulation in the region is spatially homogeneous enough to justify using the data of this gridpoint for forcing the experiments, rather than averaging over a box around the buoy. Additionally, the vertical profiles of wind, temperature and moisture above $3 \mathrm{~km}$ are nudged to ERAInterim profiles with a relaxation timescale $\tau_{a}=6$ hours. This ensures realistic evolution of the atmosphere, while leaving sufficient freedom in the marine boundary layer. ERAInterim data is also used to validate the atmosphere column simulation. Additionally, we use high temporal resolution shortwave radiation data from the buoy.

For the ocean initialisation we use daily vertical temperature and salinity profiles from the PIRATA buoy (Servain et al., 1998; Rouault et al., 2009). Temperature data is available down to $500 \mathrm{~m}$, and salinity down to $120 \mathrm{~m}$. Below these depths, we extend the profiles with monthly mean profiles from the ECMWF ocean reanalysis system ORAS4 (Balmaseda et al., 2013). These are adjusted to match the bottom temperature and salinity of the buoy data. From there the ocean evolves freely throughout the simulation, without nudging to reference profiles. Chlorophyll data from Sea-viewing Wide Field-of-view Sensor Ocean Color Data from the NASA Goddard Space Flight Center is used to take into account heating by solar penetration (Center and Laboratory, 2014).

\subsubsection{Sensitivity Experiments}

The sensitivity experiments performed for this study are listed in Table 3.1. Only settings that deviate from the control experiment are specified in the table.

First, we test the impact of atmospheric biases on SSTs. We perform a simulation in which we replace the shortwave radiation the ocean receives with observed shortwave radiation from buoy data (experiment "Shortwave").

Furthermore, to test the contribution of other surface fluxes we perform an experiment in which we nudge the horizontal wind components, as well as the temperature and moisture 
profiles from ERA-Interim down to the surface with a relaxation timescale that is equal to the model timestep (15 min, "UVTQ ERA"). In two separate experiments we nudge only the horizontal wind components to ERA-Interim profiles, and $\mathrm{T}$ and $\mathrm{Q}$ to control profiles, and vice versa (experiments "U,V ERA" and "T,Q ERA").

Secondly, we perform sensitivity experiments in which we test the intrinsic ocean contribution to the SST bias. In the absence of advection, we focus on the parameterisation of vertical turbulent mixing. Two coefficients in this scheme lend themselves for sensitivity experiments: $C_{L C}$ and $C_{d i f f}$. Both parameters are highly uncertain, due to differing measurement results by which they are constrained. As mentioned above, $C_{L C}$ is set to 0.15 as a default, but can physically be as large as 0.45 (Axell, 2002). In " $C_{L C}$ sweep" we perform a suite of sensitivity experiments in which we vary this parameter.

In the sensitivity experiment suite " $C_{\text {diff }}$ sweep" we test the impact of $C_{\text {diff }}$ by performing a sweep of single column model integrations in which we vary its value in the physical plausible possible range between 0.035 and 0.28 (see Section 3.2.1).

Lastly, we test the influence of ocean stratification on the calculation of the vertical turbulent coefficients. Stratification enters the computation of the turbulent coefficients via the Brunt-Väisälä Frequency $N^{2}$, the frequency at which a displaced mass element oscillates around its location in a static case. $N^{2}$ is used to calculate the mixing length $l_{\text {mix }}$, the distance across which the turbulent mixing can act (equal to the dissipation length scale $l_{\text {diss }}$, Eq. 3.16).

$$
l_{\text {mix }}=l_{\text {diss }}=\sqrt{\frac{2 \overline{\mathrm{e}}}{N}}
$$

Via the mixing length, $N$ enters into the calculation of the vertical eddy coefficients (Eq. 3.17). In the sensitivity experiment " $N^{2}{ }_{P I R}$ ", we test the impact of (erroneous) model stratification on the SST bias. Instead of allowing the model to calculate $N^{2}$ from its own active tracer profiles, we replace them with high temporal resolution profiles from observations. The replacement happens at the point where $N^{2}$ is calculated exclusively, and is not equivalent to ocean nudging.

\subsection{Results}

\subsubsection{Temperature bias in the Single Column Model}

During the first four days of the simulation, the SCM ensemble follows the observed cooling very well (Fig. 3.3a). In that time, sea surface temperatures cool by almost a degree in both the observations and the model. However, the daily cycle is considerably stronger in the model than in the observations. Both the daily maximum and minimum sea surface temperatures are over-/underestimated by the model. 
The South-Eastern Tropical Atlantic SST bias investigated with a coupled 54 atmosphere-ocean single column model at a PIRATA mooring site.

Table 3.1: Sensitivity experiments performed with the coupled single column model, and their overall root mean square sea surface temperature and surface shortwave radiation (SSR) biases with respect to PIRATA observation. The upper part of the table lists experiments with changes in the atmosphere, while the lower part of the table lists experiments with changes in the ocean. All experiments are performed for the period of June 1st - 30th in 2014. * For the sweeps we note the minimum RMSE at optimal parameter value.

\begin{tabular}{|c|c|c|c|}
\hline Experiment & Description & RMSE SST & RMSE SSR \\
\hline Control & $\begin{array}{l}\text { Coupled SCM, atmosphere driven } \\
\text { by T,Q,U, and V advection from } \\
\text { ERA-Interim, and relaxed above } \\
3 \mathrm{~km} \text { with } \tau_{a}=6 \text { hours. }\end{array}$ & $1.25^{\circ} \mathrm{C}$ & $103 \mathrm{~W} / \mathrm{m}^{2}$ \\
\hline Shortwave & $\begin{array}{l}\text { Coupled SCM, ocean forced with } \\
\text { shortwave radiation from PI- } \\
\text { RATA buoy observation. }\end{array}$ & $1.33{ }^{\circ} \mathrm{C}$ & $0 \mathrm{~W} / \mathrm{m}^{2}$ \\
\hline Atm ERA & $\begin{array}{l}\text { Horizontal wind components U,V } \\
\text { and } \mathrm{T} \text { and } \mathrm{Q} \text { profiles from ERA- } \\
\text { Interim nudged down to the sur- } \\
\text { face. }\end{array}$ & $0.70^{\circ} \mathrm{C}$ & $94 \mathrm{~W} / \mathrm{m}^{2}$ \\
\hline U,V ERA & $\begin{array}{l}\text { Horizontal wind components U,V } \\
\text { from ERA-Interim nudged down } \\
\text { to the surface, } \mathrm{T} \text { and } \mathrm{Q} \text { atmo- } \\
\text { spheric profiles from control sim- } \\
\text { ulation. }\end{array}$ & $1.28^{\circ} \mathrm{C}$ & $101 \mathrm{~W} / \mathrm{m}^{2}$ \\
\hline T,Q ERA & $\begin{array}{l}\mathrm{T} \text { and } \mathrm{Q} \text { profiles from ERA- } \\
\text { Interim nudged down to the sur- } \\
\text { face, } \mathrm{U} \text { and } \mathrm{V} \text { from control simu- } \\
\text { lation. }\end{array}$ & $0.69{ }^{\circ} \mathrm{C}$ & $95 \mathrm{~W} / \mathrm{m}^{2}$ \\
\hline$C_{L C}$ sweep & $\begin{array}{l}\text { Coupled SCM in different config- } \\
\text { urations as described above, with } \\
\text { varying Langmuir coefficient. }\end{array}$ & $0.83^{\circ} \mathrm{C}^{*}$ & $96 \mathrm{~W} / \mathrm{m}^{2} *$ \\
\hline$C_{\text {diff }}$ sweep & $\begin{array}{l}\text { As } C_{L C} \text { sweep, but with varying } \\
\text { coefficient } C_{d i f f} \text { for turbulent co- } \\
\text { efficient } A_{v t} \text { calculation. }\end{array}$ & $0.34{ }^{\circ} \mathrm{C}^{*}$ & $102 \mathrm{~W} / \mathrm{m}^{2} *$ \\
\hline$\overline{N^{2}{ }_{P I R}}$ & $\begin{array}{l}\text { Coupled SCM, but turbulent co- } \\
\text { efficients are calculated from PI- } \\
\text { RATA temperature and salinity } \\
\text { profiles from buoy data. }\end{array}$ & $0.89^{\circ} \mathrm{C}$ & $97 \mathrm{~W} / \mathrm{m}^{2}$ \\
\hline
\end{tabular}


Figure 3.3: Average sea surface temperature at the buoy location at $6^{\circ}$ South, $8^{\circ}$ East in June for the years 2014-2018.

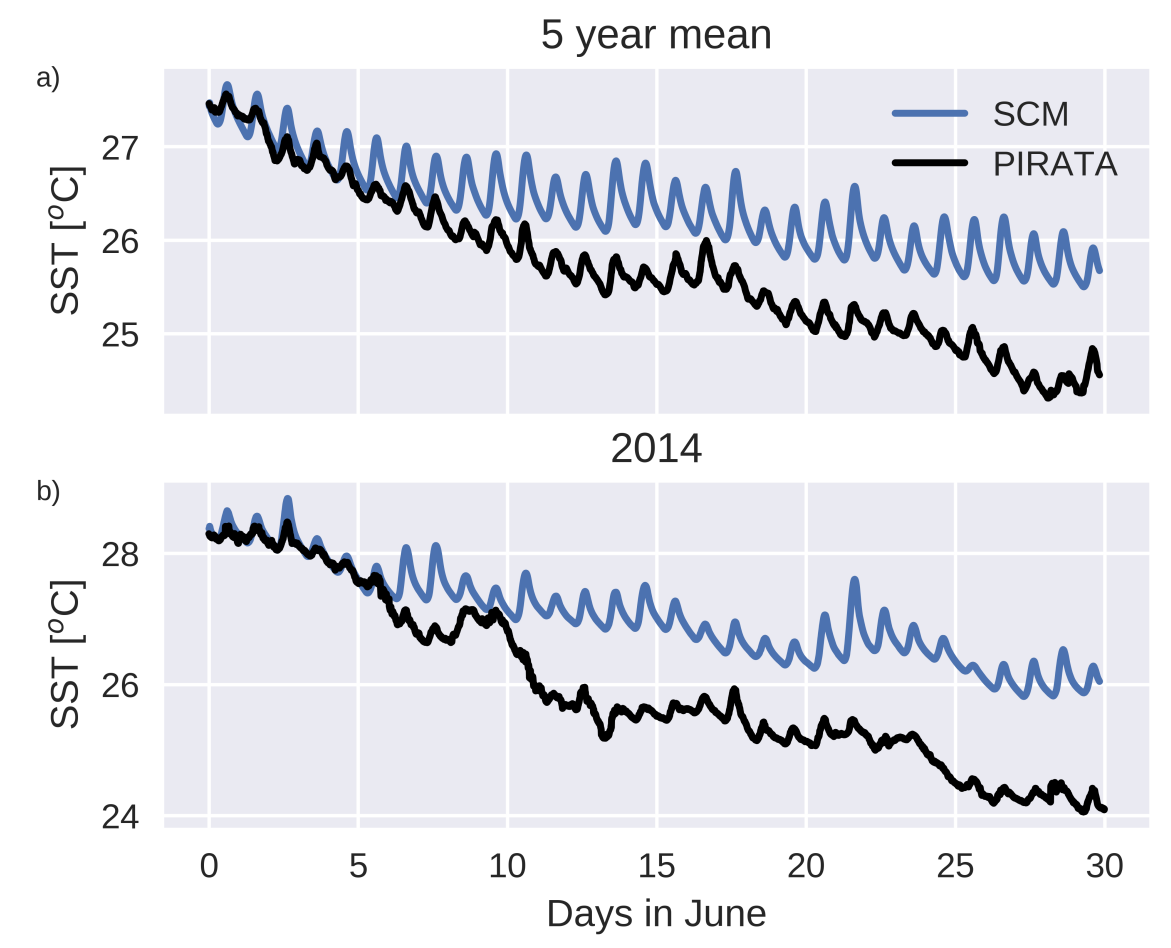

After the initial phase, observed sea surface temperature continue to decrease strongly, in total by almost three degrees at the end of the month. The model cools by less than two degrees. In a graduate build-up, the SST bias grows to $1.1{ }^{\circ} \mathrm{C}$ at the end of the simulation. This bias is smaller than that of most state-of-the-art coupled GCMs, but it is only slightly smaller than the bias in the 3D version of EC-Earth (Exarchou et al., 2017; Voldoire et al., 2019, and Fig. 3.1b). The SST bias in this region in initialised EC-Earth simulations grows to approximately one degree during June (Deppenmeier et al., under review at Climate Dynamics).

For the sensitivity experiments in this paper, we choose a year that represents the ensemble average well. In 2014, model SSTs follow observed SSTs closely during the first five days (Fig. 3.3b). Daily maximum temperatures are overestimated, much like in the ensemble average. From day six onwards, the SCM cannot reproduce the observed cooling. A warm SST bias builds up gradually, and reaches two degrees at the end of the month.

In the control simulation the SCM displays a root mean square atmospheric temperature bias of $0.81^{\circ} \mathrm{C}$ and moisture excess of $9.2 \cdot 10^{-4} \mathrm{~kg} / \mathrm{kg}$ in the lowest kilometer of the atmosphere (see comparison SCM and ERA-Interim, Fig. 3.4, upper panels). The qualitative evolution of both moisture and temperature is well captured by the model. The air cools and dries throughout June. However, the SCM the column is too warm and too moist. The wet bias is already present at the very beginning of the simulation, when sea surface 


\section{The South-Eastern Tropical Atlantic SST bias investigated with a coupled 56 atmosphere-ocean single column model at a PIRATA mooring site.}

temperatures are still very close to the ones observed. This indicates that it arises in the atmosphere. It is indeed also present in an atmosphere only (AMIP-type) simulation (not shown). The $10 \mathrm{~m}$ temperature warm bias grows with time, but the wet bias is largest in the beginning and remains relatively stable thereafter. Near-surface temperatures in an AMIP type simulation are cooler than in the coupled simulation, especially after 5-10 days of runtime (not shown).

Below the ocean surface, temperatures in the first ten meters decrease steadily throughout the month in the buoy measurements (Fig. 3.4, bottom panel left hand side). At depth between 10 and $30 \mathrm{~m}$, the measurments show very short time scale variability, leading to rapid and short-lived deepening and shallowing of the thermocline. Near-surface ocean temperatures in the SCM also decrease, but less so than in observations. In the model, the thermocline deepens monotonically and diffuses throughout the month (Fig. 3.4, bottom panel right hand side). This trend is not visible in the observational data. Similar to what we have observed for the daily cycle of SST, upper ocean temperature in the SCM displays a stronger diurnal cycle than the buoy data. In the model, a shallow warm near-surface layer of up to $10 \mathrm{~m}$ depth develops every day.

The control simulation surface fluxes show of a relatively constant shortwave radiation input, and similarly constant radiative and turbulent cooling (Fig 3.5). In total, the SCM surface fluxes warm the ocean. The observed cooling therefore must be a result of cooling from below, which in the absence of advection must be caused by vertical turbulent entrainment of cold water into the warm surface layer.

To summarise, the single column model performs very well during the first days of the simulation. Thereafter, it rapidly develops a sea surface temperature bias very similar to that of its 3D counterpart. Atmospheric moisture is overestimated from the beginning of the simulation, near surface atmospheric temperatures increase simultaneously with the SST bias.

In the following, we investigate different reasons for the model biases and possibilities to alleviate them. First, we focus on impacts arising in the atmosphere in section 3.4.2, and then on the ocean model itself in section 3.4.3.

\subsubsection{Surface forcing}

A possible explanation for the warm sea surface temperature bias could be excess shortwave radiation, which artificially heats the sea surface. This has been suggested for the eastern boundary region in the Pacific (Ma et al., 1996a), and might be true also for the Atlantic (Huang et al., 2007; Hu et al., 2011; Zuidema et al., 2016). We investigate this possibility with two approaches. First, we compare the surface shortwave radiation from the SCM to the buoy measurements, to establish whether a positive shortwave radiation bias is present. 
Figure 3.4: June 2014 atmosphere specific humidity (top row), atmospheric temperature (centre row) and ocean temperature (bottom row) at the buoy location at $6^{\circ}$ South, $8^{\circ}$ East in June, atmospheric data from ERA-Interim and ocean temperature from PIRATA data, and as modelled by the single column model (control). Ticks on the right hand sides of the bottom row indicate levels at which ocean data is available. Atmospheric data is available on the same model level heights for ERA-Interim and the SCM.
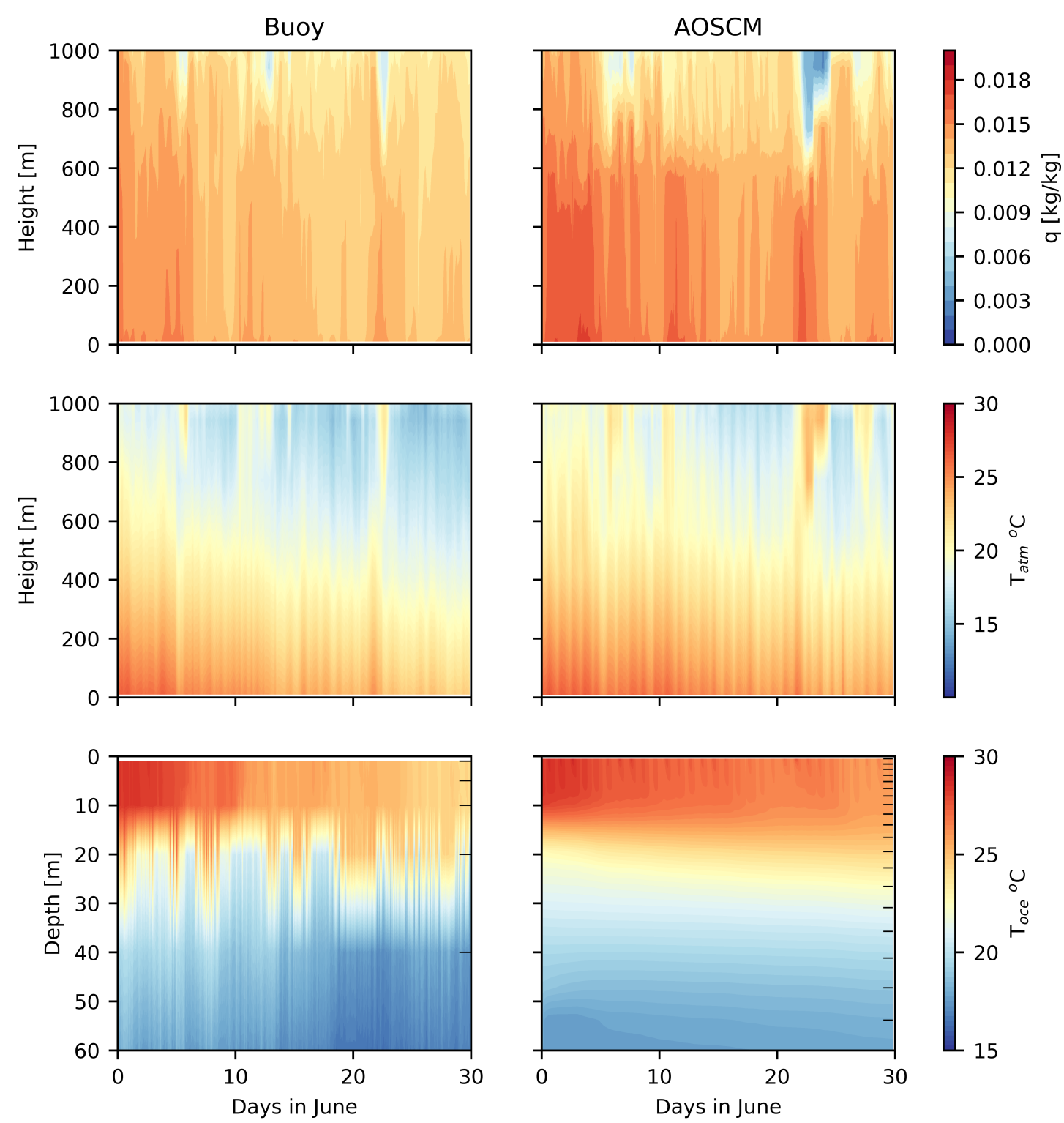


\section{The South-Eastern Tropical Atlantic SST bias investigated with a coupled 58 atmosphere-ocean single column model at a PIRATA mooring site.}

Figure 3.5: Control simulation (June 2014) surface fluxes, net shortwave (red, SW), net longwave (blue, LW), and the turbulent fluxes latent heat and sensible heat combined (green, $\mathrm{LH}+\mathrm{SH})$. Of the latter, latent heat is the much larger contribution. The sum of the surface fluxes is shown in black (TOT).

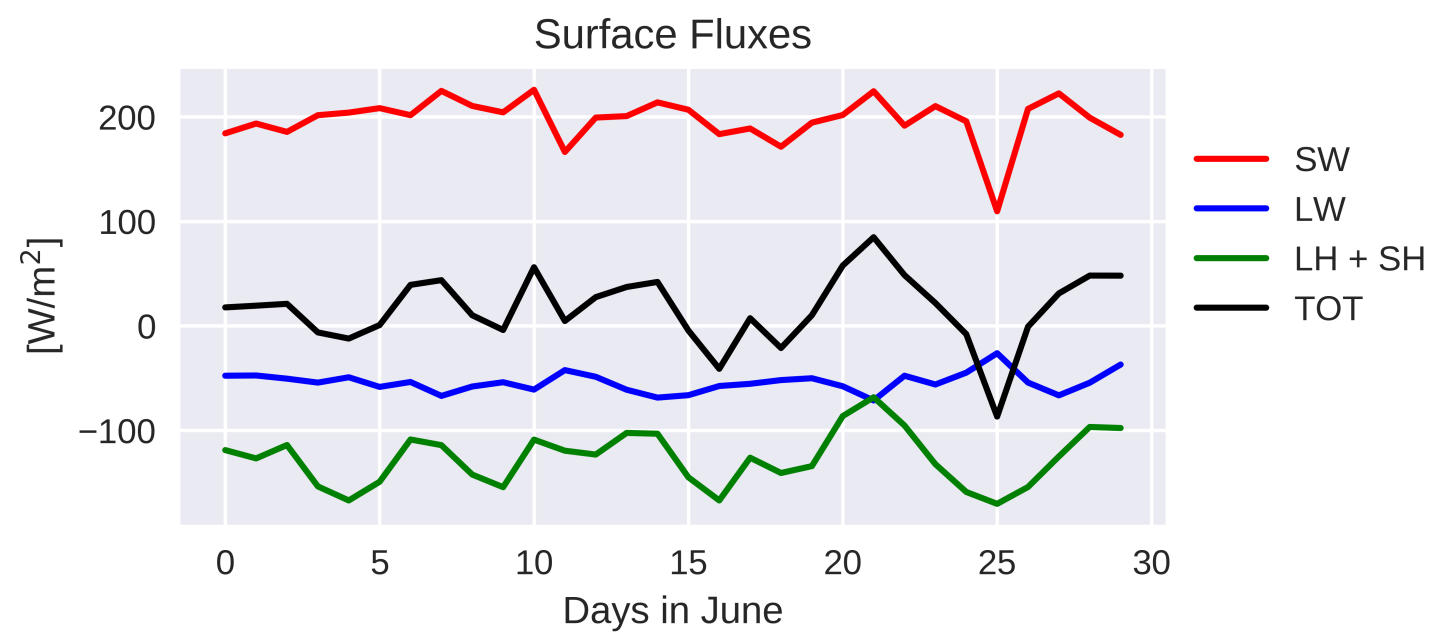

At first glance, the surface shortwave radiation time series of the SCM seems to suffer from a shortage of radiation rather than a surplus on most days in the simulation (Fig. 3.6). However, the two data sets cannot readily be compared, because of their differing time resolution. Model data is available on 15 minute intervals, while buoy data is provided at 2 minute intervals. To eliminate the apparent differences arising from differences in temporal resolution, and therefore differences in the representation of intermittency, we compute daily integrals of shortwave radiation (Fig. 3.7). The integrated daily amount of surface short wave radiation in the SCM is very similar to the one observed. The difference between the total energy input during the length of the simulation depends only slightly on whether the original 2-minute data from PIRATA is used or whether PIRATA data is interpolated to the 15 minute resolution of the SCM. In the latter case, energy input between the model and the observations only differs by $0.05 \%$. In the former case the energy difference amounts to $0.5 \%$ excess in the SCM as compared to PIRATA, $2382 \mathrm{~kJ}$ over the entire month. This difference is due to the high intermittency of observed surface radiation, which cannot be matched by the SCM output frequency. However, the excess shortwave radiation cannot account for the SST bias. An estimate of the SST tendency term due to heating $\frac{\partial T}{\partial t}=\frac{Q}{h \cdot \rho_{w} \cdot c_{p}}$, with an assumed sea water density $\rho_{w}$ of $1020 \mathrm{~kg} / \mathrm{m}^{3}$ and a specific heat capacity $c_{p}$ of $4000 \mathrm{~J} / \mathrm{kg} / \mathrm{K}$ and a very shallow mixed layer depth $h$ of $20 \mathrm{~m}$ shows that the order of magnitude of heating due to this excess is $0.03^{\circ} \mathrm{C}$. The surface solar radiation bias, hence, cannot explain the warm sea surface temperature bias, which is larger by almost two orders of magnitude. This conclusion is consistent with those of other studies using EC-Earth (Exarchou et al., 2017; Voldoire et al., 2019; Deppenmeier et al., under review at Climate Dynamics). 
Figure 3.6: Downward shortwave radiation from the PIRATA buoy and from the single column model control run, at 2 minute and 15 minute temporal resolution, respectively.

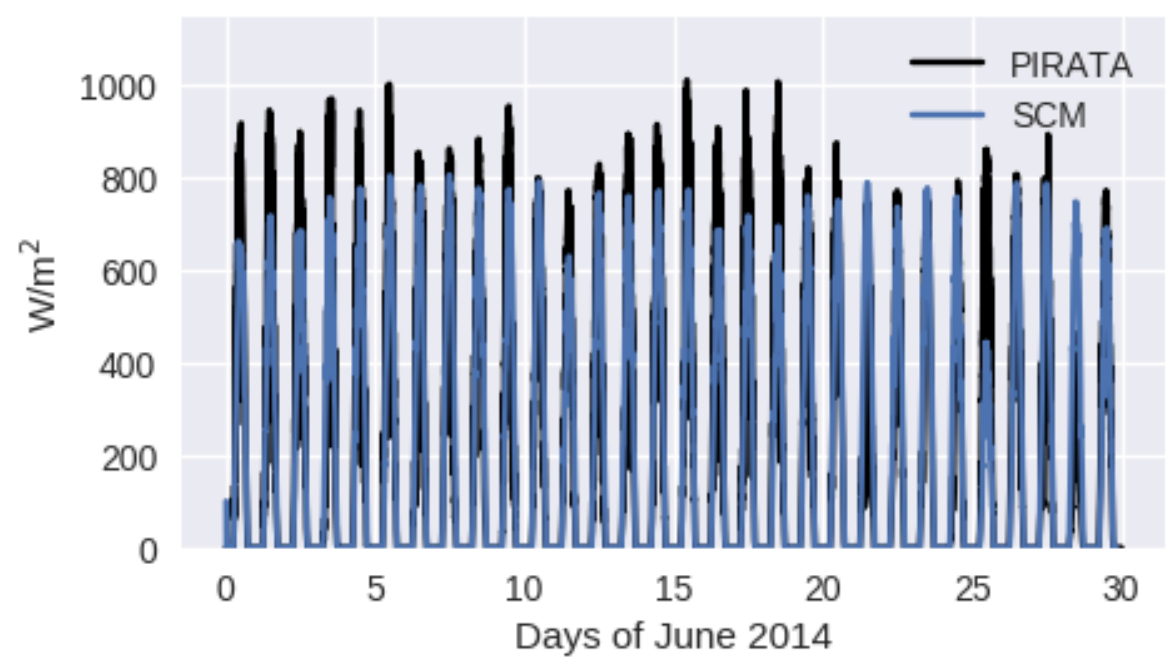

Figure 3.7: Daily integrals of the downwards shortwave radiation flux during June from the PIRATA buoy, from data received at 2 minute intervals, and interpolated to model timestep resolution of 15 minutes, and from the single column model control run.

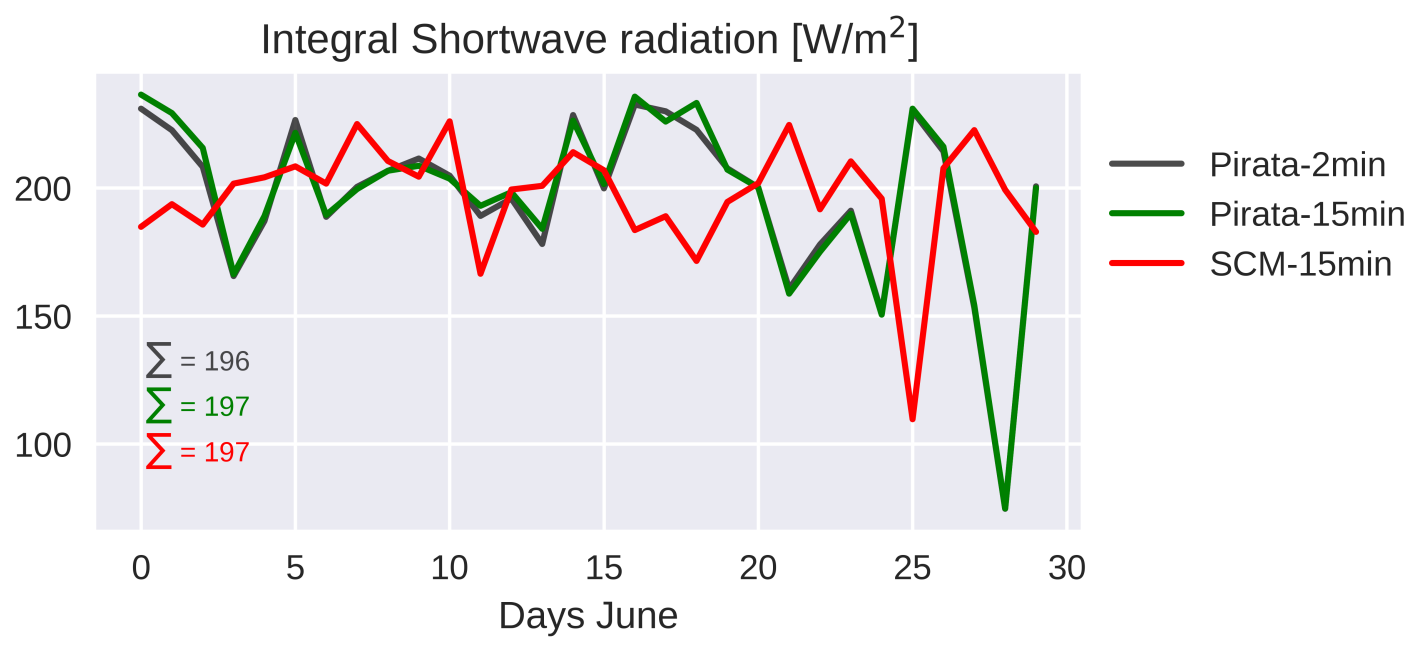




\section{The South-Eastern Tropical Atlantic SST bias investigated with a coupled 60 atmosphere-ocean single column model at a PIRATA mooring site.}

Figure 3.8: Sea surface temperature as simulated by the various atmospheric experiments described in the upper part of Table 3.1. The black line shows observed SST for comparison.

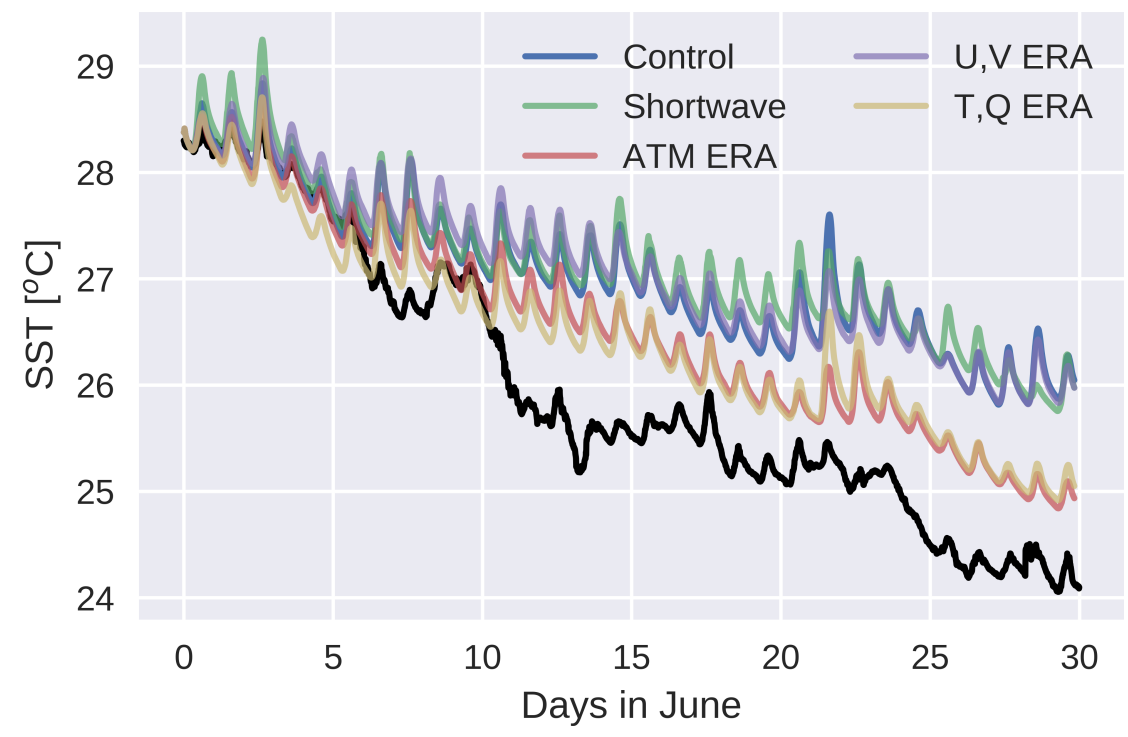

Atmospheric Sensitivity Experiments

Even though the difference in surface shortwave radiation is small, feedbacks involving in it could still influence the SST bias. To test this hypothesis, we perform a coupled simulation in which the ocean receives observed shortwave radiation, instead of the one calculated by the atmospheric component (Experiment "Shortwave"). Consistent with the conclusion from the shortwave radiation analysis, the SST bias in this simulation does not reduce. The sea surface temperature evolution is hardly influenced during the time of the simulation (Fig. 3.8, green line). This solidifies the notion that surface shortwave radiation is not the main origin of the warm SST bias in the south eastern part of the cold tongue.

In Section 3.4.1, we have seen that the near surface atmosphere in the SCM is warm and wet biased. Both these biases could be a cause or consequence of the warm SST bias. To determine the impact of atmospheric biases on the SST bias, we investigate sensitivity experiments in which the atmosphere is unbiased (with respect to ERA-interim). In this experiment, "Atm ERA", the sea surface temperature bias reduces from $1.25{ }^{\circ} \mathrm{C}$ to $0.69{ }^{\circ} \mathrm{C}$. The observed cooling now matches for approximately 10 days of the simulation (Fig. 3.8, red line). After that, the steep observed cooling can, again, not be reproduced by the model.

The reduction of SST bias is notable, however, and we investigate the cause further. A possible reason for the warm SST bias originating in the atmosphere is reduced forcing of the ocean due to underestimated winds. This theory has recently been supported by $\mathrm{Xu}$ 
et al. (2014b) and Voldoire et al. (2019). We test the influence of wind biases on the SST bias in experiment "U,V ERA". The wind forcing hardly impacts the simulation (Fig. 3.8, purple line). The SST root mean square error decreases only by $0.05{ }^{\circ} \mathrm{C}$. Voldoire et al. (2019) show EC-Earth to be the least sensitive CGCM to the wind stress replacement. This is due to the small wind stress bias in the model compared to ERAInterim. In the SCM, the wind stress bias is also small. As a consequence, wind stress nudging mostly changes the direction of the wind, but does not enhance its amplitude (not shown). Because the wind bias is small to begin with, this experiment does not much impact the surface flux budget (Fig. 3.9, panel b), and hence has a very small impact on the RSME SST.

The atmosphere furthermore exerts influence on the ocean surface via surface level temperature and moisture, which impact the surface flux budget. It has recently been suggested that atmospheric moisture is a major cause for the warm SST bias (Hourdin et al., 2015). In the experiment "T,Q ERA" we remove the model bias of temperature and moisture with respect to ERA-Interim. This experiment is able to almost reproduce the cooling of "Atm ERA". The SST root mean square error (RMSE) in this simulation is reduced to $0.69{ }^{\circ} \mathrm{C}$.

In both experiments in which moisture and temperature are adjusted an increase in turbulent surface fluxes drive the SST cooling by reducing the total surface flux going into the ocean (Fig. 3.9). The turbulent fluxes cool considerably more when atmospheric temperature and moisture are improved. The total surface flux in the sensitivity experiments with the latter variables from ERA-Interim even changes signal, and now cools the ocean rather than warming it, as in the control.

We have demonstrated the impact of the (near surface) atmosphere conditions as well as shortwave radiation on the single column model sea surface temperature bias. While shortwave radiation is modeled accurately at the location of the buoy, the warm and moist near surface air bias contribute to the warm SST bias. In bias-reduced atmosphere simulations it is possible to reduce the root mean square SST bias from $1.25^{\circ} \mathrm{C}$ to $0.69{ }^{\circ} \mathrm{C}$. This is a considerable reduction, but a sizeable SST bias remains, even if the atmosphere is unbiased. The origin of the remaining bias lies in the ocean interior. Hence, in the following section, we will turn our attention to the ocean.

\subsubsection{Ocean model}

Entrainment of cold water by turbulent vertical mixing plays a large role in the tropical upper ocean heat budget (Foltz et al., 2003; Moum et al., 2013; Hummels et al., 2014; Scannell and McPhaden, 2018). If this process is underrepresented, due to, for example, inadequate parameterisation, it can lead to warm SST biases. Too little cold water could be entrained into the shallow mixed layer from below, leading too insufficient cooling. In 
The South-Eastern Tropical Atlantic SST bias investigated with a coupled 62 atmosphere-ocean single column model at a PIRATA mooring site.

Figure 3.9: Panel a) shows surface fluxes as in Fig. 3.5, net surface shortwave radiation in red, net surface longwave in blue, turbulent fluxes in green, and total sum of fluxes in black. The control experiments is depicted in solid faded lines, Atm ERA in dashed lines, T,Q ERA in dotted lines, and U,V ERA in dashed-dotted lines. Panel b) shows the relative change of integrated surface fluxes with respect to the control experiment, in percent. Colours as in panel a). Note that due to the sign convention an increase in turbulent as well as longwave radiative flux translates to an increased in cooling.

a)

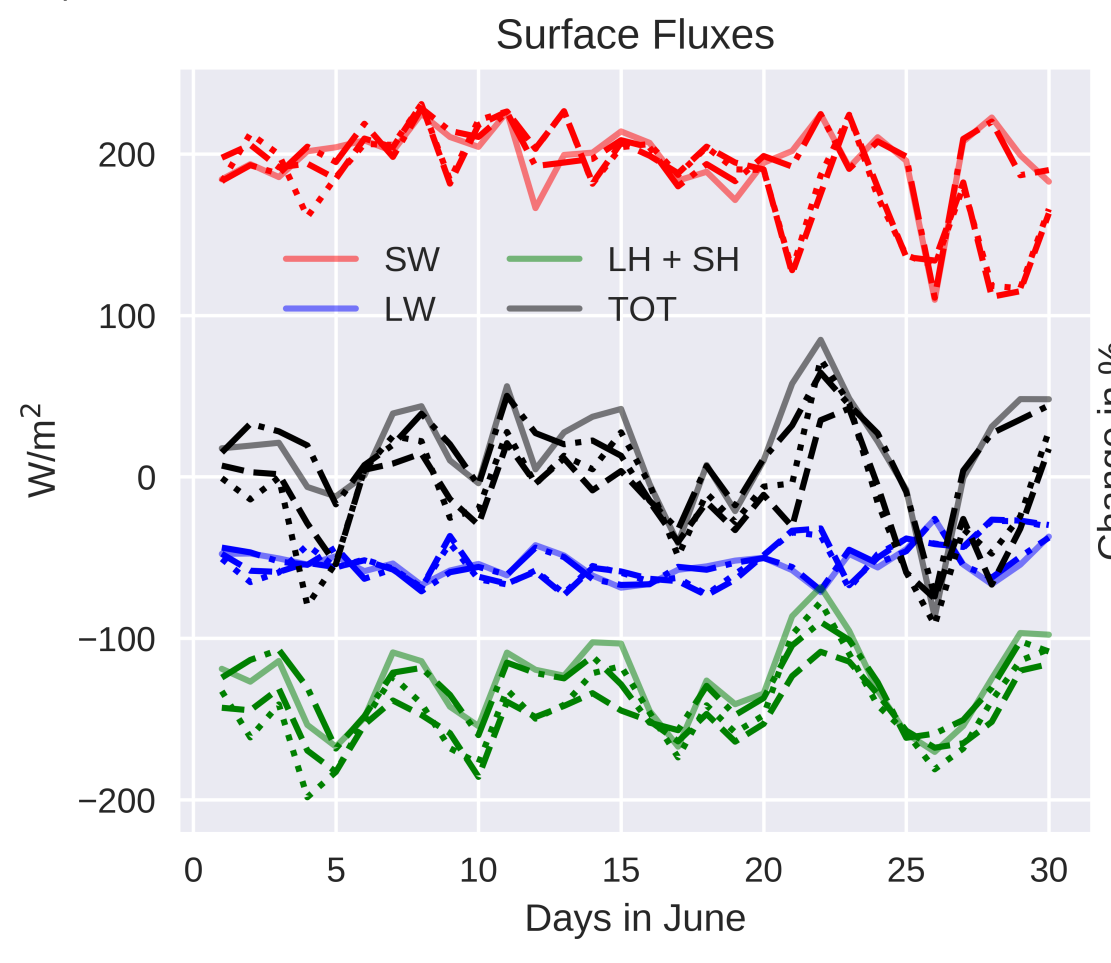

b)

\section{Relative Changes}

15

10

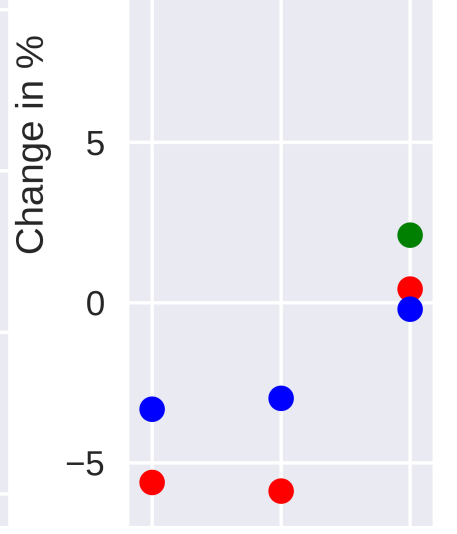

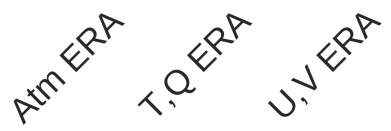


this section we examine influences on vertical turbulent mixing in the upper ocean and their effect on the sea surface temperatures.

The TKE scheme, as described in Section 3.2.1, adds the contributions to the available turbulent kinetic energy, and then infers the turbulent mixing coefficients, which determine mixing in the ocean column. The first source term of TKE is the Langmuir Cell parameterisation.

\section{Langmuir circulation}

Langmuir circulation is dependent on wind input at the surface, and the stability of the ocean column. Langmuir circulation can be an important contribution to entrainment by cool water at the bottom of the mixed layer (Skyllingstad and Denbo, 1995). They appear generally above wind speeds of $3 \mathrm{~m} / \mathrm{s}$ (Talley, 2011), which is frequently crossed in our simulations (not shown). The strength of the parametrised circulation is dependent on the coefficient $C_{L C}$, which has been set to 0.15 by (Axell, 2002). Its value can be increased, but the recommendation is to keep it below 0.54 . Here, we test the whole parameter space between the two values.

Overall, there is a slight decrease in root mean square SST bias with increasing Langmuir coefficient (Fig. 3.10, green markers). It is notable, however, that the RMSE between values of $C_{L C}=0.15$ and 0.45 are noisy, rather than showing a clear tendency. SST RMSE only decreases more consistently at values larger than 0.45 . From the shape of the curve no clear recommendation can be made for the value of $C_{L C}$, though higher values might be preferred, rather than the very low default value.

\section{Vertical mixing efficiency}

Next, we test the response to increasing the vertical mixing efficiency in the turbulent kinetic energy scheme. The vertical mixing coefficients $A_{v t}$ and $A_{v m}$ depend on $C_{d i f f}$ that represents the ocean mixing efficiency (Equations 3.17, 3.18, and 3.19). This factor is losely constrained by measurements, but can assume values between 0.035 and 0.28. Here, we test the parameter space in the same manner as in Section 3.4.3.

The root mean square error (RMSE) of SST is very sensitive to the value of $C_{d i f f}$ (Fig. 3.10, blue markers). At the default value $C_{d i f f}=0.1$, the RMS SST bias has a value of $1.25^{\circ} \mathrm{C}$. At lower values, i.e., at less efficient mixing, the bias is even larger (up to $1.87^{\circ} \mathrm{C}$ at lowest $C_{d i f f}=0.035$ ), growing with decreasing $C_{d i f f}$. Sea surface temperature RMSE values decrease rapidly with increasing $C_{d i f f}$. The minimum bias is reached at $C_{\text {diff }}=0.23$, the bias then amounts to only $0.32^{\circ} \mathrm{C}$. This is a reduction of $74 \%$ of the default bias. Between values of 0.2 and 0.25 for $C_{d i f f}$, the bias is relatively stable and very low. When $C_{i f f}$ is increased further, the root mean square error of SST increases 


\section{The South-Eastern Tropical Atlantic SST bias investigated with a coupled 64 atmosphere-ocean single column model at a PIRATA mooring site.}

Figure 3.10: Root mean square sea surface temperature errors in the parameter sweeps for $C_{L C}$ and $C_{d i f f}$.

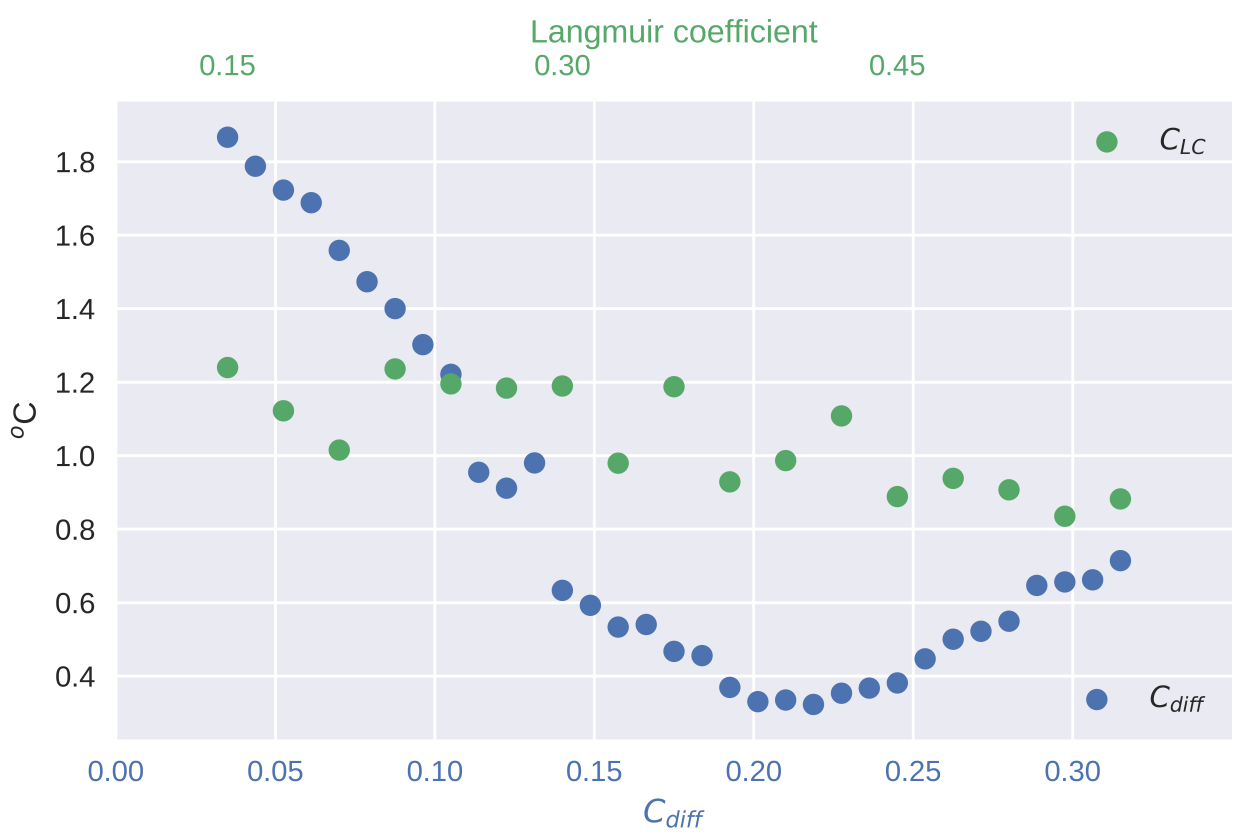

again. This is due to the model sea surface then becoming too cold, leading to a cold bias with respect to observations.

At the optimal value for $C_{d i f f}$, model sea surface temperatures follow observations well (Fig. 3.11, purple line). Enhancing turbulent vertical mixing in the ocean column within the physically plausible range can reduce the SST bias to approximately a quarter of its original amplitude.

In the ocean column, the warm top layer formation is reduced with the optimal $C_{d i f f}$ as compared to the control (Fig. 3.12, bottom row), but not entirely removed. The diurnal cycle remains too strong compared to observations (as is also evident from sea surface temperatures (Fig. 3.11)). Short timescale subsurface temperature variability as observed in PIRATA data (Fig. 3.4) is not present in the model, despite the increased vertical mixing activity.

Consistent with the cooler sea surface temperatures, near surface atmospheric temperatures are also decreased. However, higher up the atmosphere strongly warms as compared to the control (Fig. 3.12, centre row). The atmosphere was warmer than ERA-Interim to begin with (Fig. 3.4), hence, this is a degradation of model performance. The overstimation of near surface moisture is increased near the surface, and aloft a dry region forms that is not observed in ERA-Interim.

Surface fluxes along the $C_{\text {diff }}$ sweep decrease (Fig. 3.13). The higher $C_{\text {diff }}$, the cooler the sea surface, and, as a consequence, both the turbulent fluxes and the longwave radiation 
Figure 3.11: Sea surface temperature as simulated by the various oceanic experiments described in the lower part of Table 3.1. The black line shows observed SST for comparison.

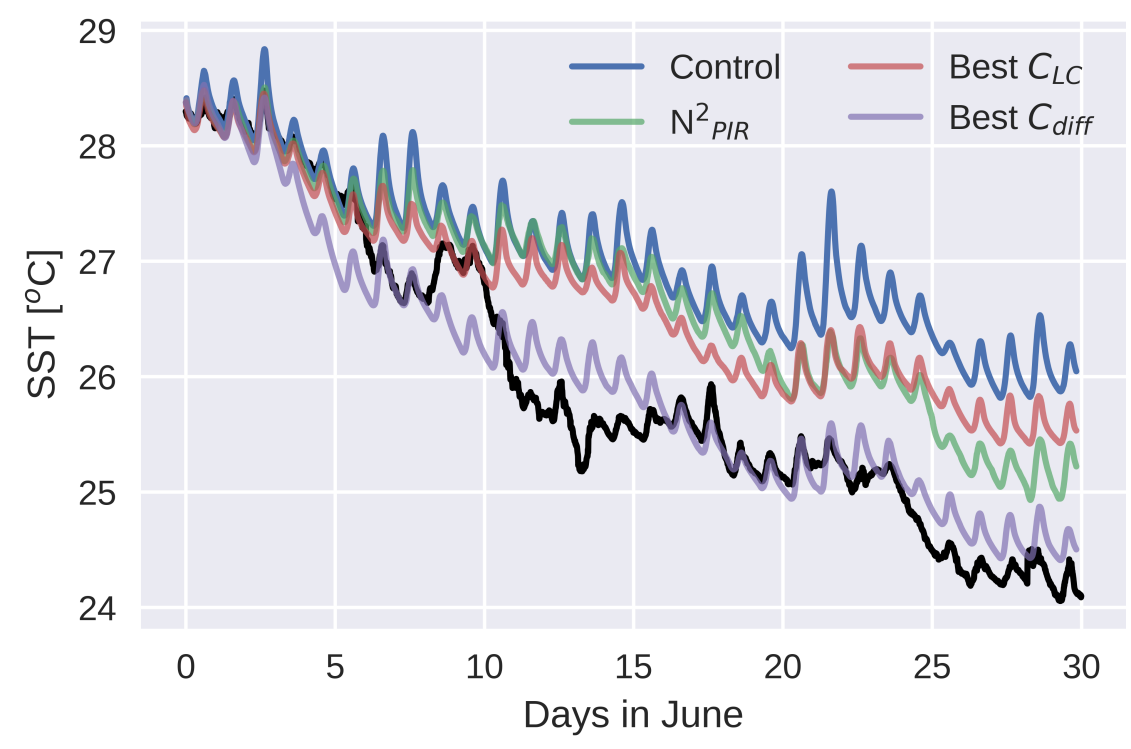

flux decrease. Shortwave radiation also decreases, though it is more complicated to place this decrease. Cooler surface temperatures might lead to more stratocumulus cloud cover due to increased boundary layer stability, but the response of clouds to the sea surface temperature is very noisy. The dominant cloud type in the simulations are shallow convection cumulus, which react strongly to perturbations of SST in AMIP type runs due to their chaotic nature (not shown). Considering that shortwave radiation is not overstimated in the control (Fig. 3.7), the reduced shortwave radiation with increased $C_{\text {diff }}$ is not a model improvement. However, it positively influences the sea surface temperature bias.

\section{Vertical mixing efficiency in changed setting}

We have demonstrated the beneficial effect of increasing the vertical ocean mixing on reducing RMSE SST. Setting $C_{\text {diff }}$ to a value twice as large as the default value, but still within the plausible physical range, results in a realistic simulation of sea surface temperatures. We have also demonstrated beneficial impact of correctly modelled near surface temperature and moisture in the atmosphere. We wonder whether already improved simulations, as "Atm ERA", are less sensitive to the value of $C_{\text {diff }}$. Therefore, we perform another set of parameter sweeps along the values of $C_{\text {diff }}$ for the nudged atmosphere experiment, and additionally for the shortwave forcing experiment.

Fig. 3.14 shows sea surface temperature RMSE depending on the value of $C_{\text {diff }}$. The RMSE have been fitted with a cubic function, stars mark minimum values on the curve. 
The South-Eastern Tropical Atlantic SST bias investigated with a coupled 66 atmosphere-ocean single column model at a PIRATA mooring site.

Figure 3.12: Atmospheric moisture (upper row), temperature (centre row), and upper ocean temperature (bottom row) in the control experiment and at ideal $C_{d i f f}$.
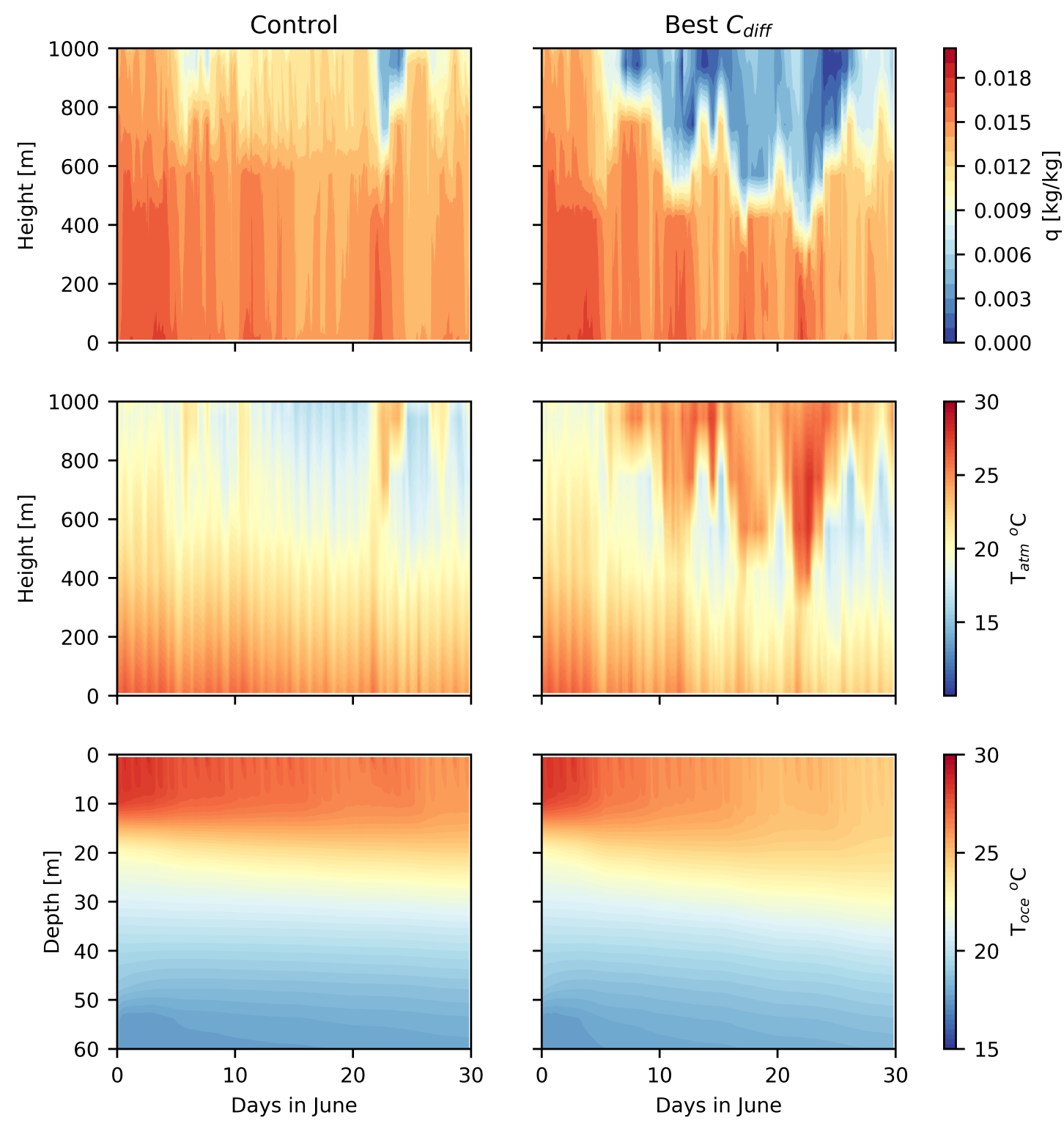
Figure 3.13: Integrated surface fluxes over the time of the simulations in the $C_{\text {diff }}$ sweep. Turbulent components latent and sensible heat fluxes are combined in green, the radiative components shortwave and longwave are in red and blue, respectively.

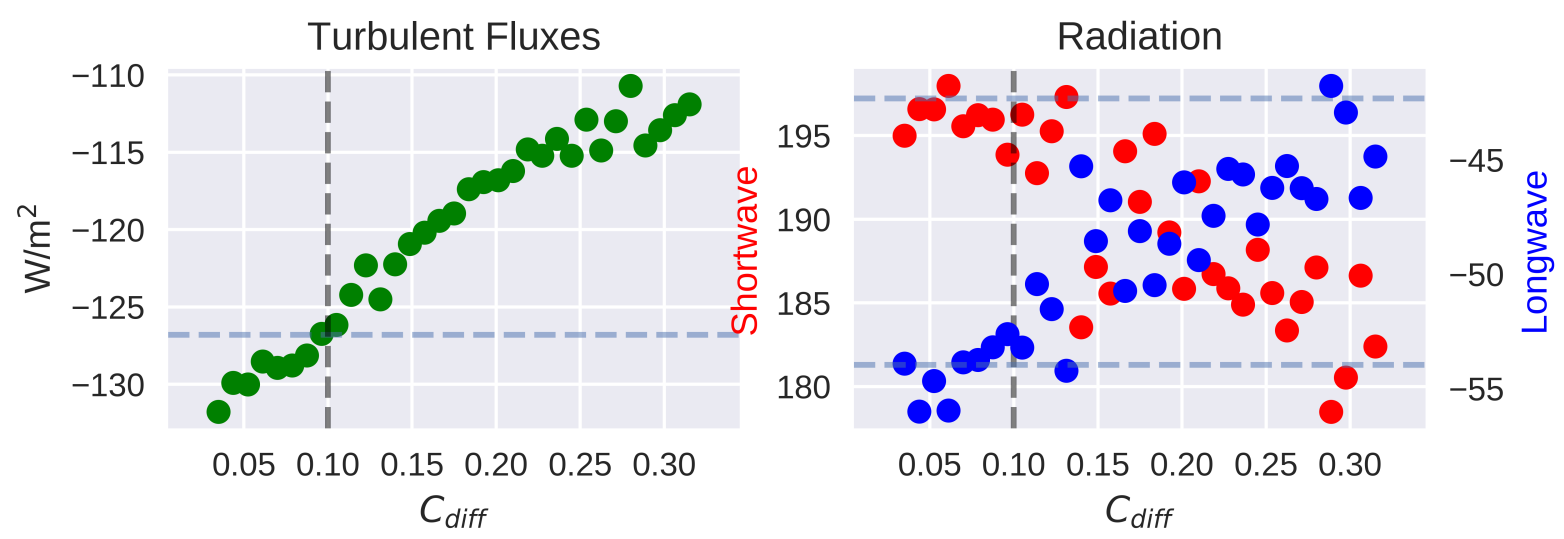

All three sweeps reach a minimum in the $C_{\text {diff }}$ range between 0.2 and 0.25 , and the RMSE at their respective ideal $C_{\text {diff }}$ are very similar. The lowest RMSE value is obtained when the atmosphere is nudged to the surface (Fig. 3.14, red). This suite is also least dependent on the value of $C_{d i f f}$, as is indicated by the relatively flat curve. Since the sea surface is already beneficially influenced by cooler near surface temperatures in the atmosphere, the SST bias does not reach values as high as in the control.

All three curves, whether it is the coupled simulation, the atmosphere nudged to the reanalysis state, or the shortwave radiation used from observations, reach their minimum around the same value of $C_{d i f f}$, between 0.20 and 0.25 (Fig. 3.14). The SST biases obtained at these $C_{d i f f}$ values are very small; meaning that the summer time SST cooling is well represented. This highlights the dominant effect of the vertical turbulent mixing on the ocean cooling. When there is enough vertical mixing, other factors, such as an improved atmosphere, does not much improve the simulation anymore. This result is hence a very strong indication of the importance of vertical turublent mixing, and implies that the value for $C_{\text {diff }}$ should be increased in $3 \mathrm{D}$ simulation. Although the mean bias reduces, the intermittency of the ocean is not captured.

\section{Stratification}

We have established in Section 3.4.1 that modelled sea surface temperatures as well as the upper $10 \mathrm{~m}$ of the ocean column display a stronger daily cycle than observed (Figures 3.4 and 3.8). This warm top layer artifically stabilises the ocean column. Ocean stability enters the turbulent kinetic energy scheme in the form of the Brunt-Väisälä frequency $N^{2}$ with a negative sign (Eq. 3.14). Increased stratification reduces the turbulent kinetic 


\section{The South-Eastern Tropical Atlantic SST bias investigated with a coupled 68 atmosphere-ocean single column model at a PIRATA mooring site.}

Figure 3.14: Root mean square sea surface temperature errors in the parameter sweeps for $C_{d i f f}$, with cubic fits. Stars mark the minimum SST RMSE on the fit.

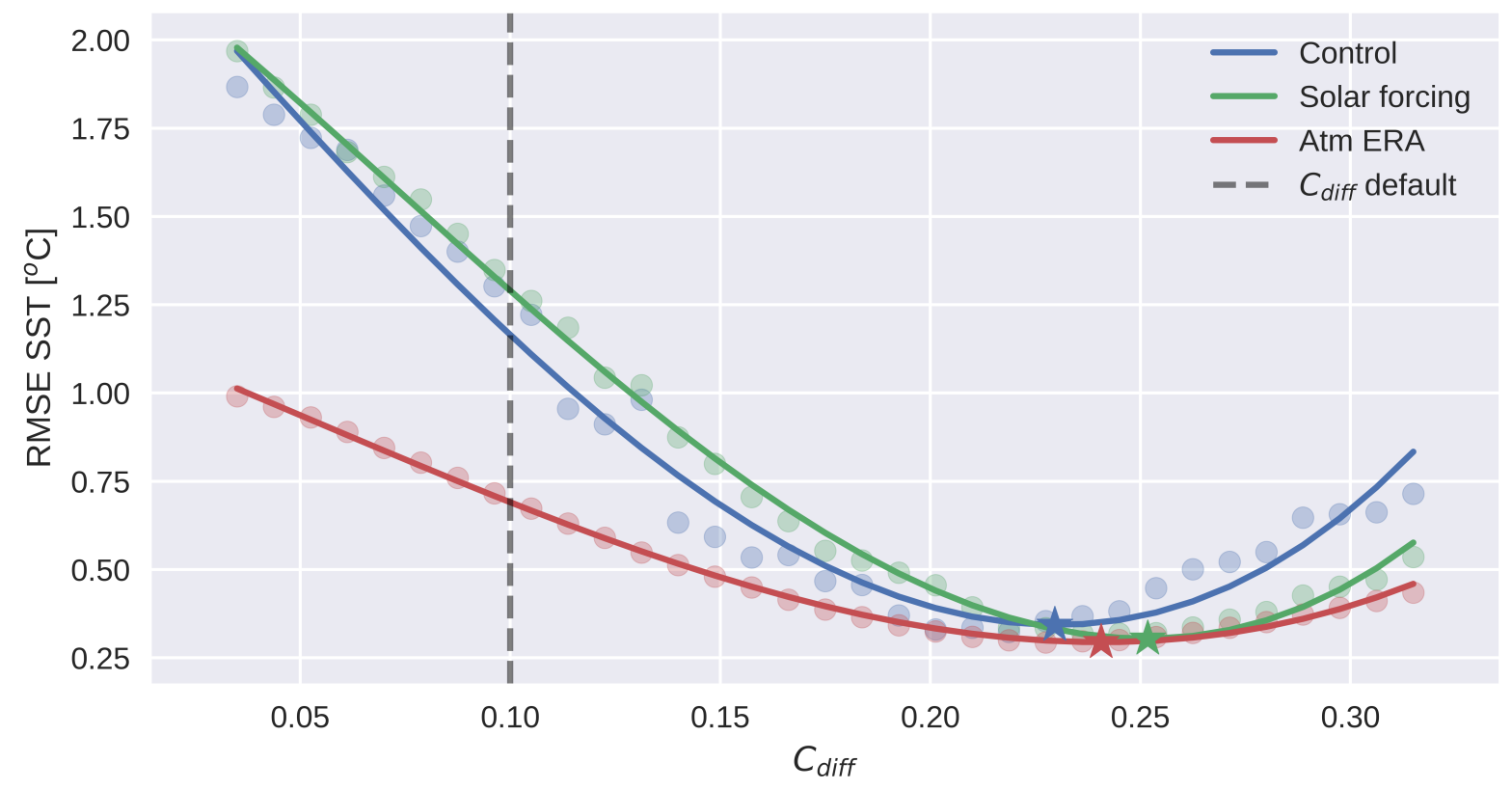

energy available to create vertical ocean mixing. Furthermore, the stability enters into the calculation of the mixing length $l_{\text {mix }}\left(\right.$ Eq. 3.16, and $l_{\text {mix }}=l_{\text {diss }}$ ). When $\bar{e}$ decreases due to large $N^{2}, l_{m i x}$ decreases. Additionally, $l_{m i x} \propto \frac{1}{N}$, the mixing length decreases even more. $l_{\text {mix }}$ enters into the calculation of the turbulent eddy coefficients via Eqs. 3.17 and 3.18. All of these effects cause vertical ocean mixing to decrease with increasing stratification.

Here, we test the effect of stratification on the ocean vertical mixing and on the SST bias. We replace model temperature and salinity with those from high temporal resolution PIRATA observations, which are less stratified. As a result, turbulent vertical mixing is increased (not shown). The enhanced mixing leads to a considerable reduction of the SST RMSE from $1.25{ }^{\circ} \mathrm{C}$ in the control to $0.89^{\circ} \mathrm{C}$ in $N^{2}$ PIR. The sea surface temperature bias is decreased by almost a third. This highlights the impact stratification assers on ocean vertical mixing. In the single column model, a positive feedback loop involving stratification and vertical mixing likely grows the SST bias. Vertical mixing is insufficiently strong in the beginning, which leads to increased stratification. The ocean stability in turn reduces the turbulent kinetic energy, which further decreases vertical mixing.

In the sensitivity experiment, $A_{v t}$ is less intermittent in the upper $5 \mathrm{~m}$ than in the control simulation (not shown). The continuously active mixing also occasionally penetrates the upper $10 \mathrm{~m}$ of the column, and towards the end of the simulation (day 25) displays very short term, but strong bursts in the upper $20 \mathrm{~m}$. The effect of the enhanced mixing 
activity on sea surface temperature is large (Fig. 3.11, green line). Especially after day 25 , when "deeper" mixing bursts first occur, the sea surface cools considerably more than in the control simulation.

\subsection{Discussion and Summary}

In this study, we use a coupled ocean atmosphere single column model to investigate the warm sea surface temperature bias in the tropical Atlantic ocean. Such a bias establishes rapidly in three dimensional coupled global circulation models throughout boreal summer. The warm bias is typically large in the south east tropical Atlantic and occurs in most coupled global climate models.

We place the SCM at a PIRATA mooring location in the south eastern tropical Atlantic. This enables us to compare the model simulation to in-situ point observations. For the average of the five years in which high temporal resolution buoy data are available, the single column version of EC-Earth performs well in the first five days of the simulation. It then produces a sea surface temperature bias very similar to that in the $3 \mathrm{D}$ version of the model, the root mean square value of the bias is $1.25^{\circ} \mathrm{C}$. This makes the SCM a useful tool to investigate the origin of the bias and test possible ways to alleviate it.

For the case of 2014 we eliminate solar surface radiation as the main cause of the warm SST bias. This is in line with other studies (Exarchou et al., 2017; Voldoire et al., 2019; Deppenmeier et al., under review at Climate Dynamics). Forcing the ocean with observed surface shortwave radiation does not improve simulation of the sea surface temperature. Note that the location in this study coincides with the trade cumulus region. Further south-east, radiation may contribute ir even be a main cause of model biases. Near surface temperature and moisture in the atmosphere, however, assert a considerable influence on simulated SSTs (producing a root mean square bias of $0.70^{\circ} \mathrm{C}$, a reduction of $44 \%$ ). Nudging winds to ERA-Interim profiles, on the other hand, hardly affects the sea surface temperature. This is to be expected for EC-Earth, which has a relatively small wind bias (Voldoire et al., 2014).

While correcting the atmosphere improves the sea surface temperature simulation, a sizeable bias remains. We show that the bias can be reduced to a quarter of its original size by making physical changes in the ocean model alone. We increase the factor with which kinetic turbulent kinetic energy is transformed to turbulence within its physical range by setting the vertical mixing efficiency coefficient $C_{\text {diff }}$ from its default value 0.1 to its optimal value 0.23 . This reduces the $\mathrm{SST}$ bias to $0.34^{\circ} \mathrm{C}$, the largest reduction we are able to achieve with any sensitivity experiment. Using the optimal value for $C_{d i f f}$ also improves the vertical ocean profile. A very stable and warm upper bias, visible in the control simulation, is reduced. However, the intermittency in observations is not captured, even 


\section{The South-Eastern Tropical Atlantic SST bias investigated with a coupled 70 atmosphere-ocean single column model at a PIRATA mooring site.}

in the experiments with highest mixing efficiency parameters. The large improvement of the ocean simulation with increased vertical mixing hints to vertical mixing being an underrepresented process in the single column model. Similarly, it is likely underestimated in the global climate models, which use the same vertical mixing parameterisation.

We test the influence of the Langmuir circulation coefficient. The model sensitivity to this parameter is much smaller than that to $C_{d i f f}$, and there is no clear optimal value for $C_{L C}$.

Furthermore, calculating vertical eddy coefficients with the correctly stratified profiles reduces the sea surface temperature bias to $0.89^{\circ} \mathrm{C}$. Upper ocean mixing is increased with the correct profiles, which reduces the SST bias. The artifical stable stratification in the control simulation leads to decreased mixing, which in turn leads to more stable stratification. This is a positive feedback that worsens the bias. Most likely, initial ocean vertical mixing is too low in the model, which then leads to the artificially stable column. This could hence be alleviated with an increase in the mixing efficiency.

In further experiments, we have also tested the maximum solar penetration depth, which has recently been suggested to assert large influence on sea surface temperature (Exarchou et al., 2017). In this study, however, we find no reduction of the bias by increasing the depth from $23 \mathrm{~m}$ to either $30 \mathrm{~m}$ or $50 \mathrm{~m}$.

In this study, we demonstrate that both the atmosphere and the ocean contribute to the warm sea surface temperature bias in the south-eastern tropical Atlantic. We show that the bias can be considerably reduced by enhancing the vertical ocean mixing efficiency within its physically plausible range. More observations to better constrain the parameter $C_{\text {diff }}$ are desirable, so that it can be confirmed whether the larger value is indeed more appropriate for modeling ocean vertical mixing. 


\section{Chapter 4}

\section{The effect of vertical ocean mixing efficiency on the tropical Atlantic in a coupled global climate model.}

This chapter is based on:

Deppenmeier, A.-L., Haarsma, R. J., LeSager, P., and Hazeleger, W.: The effect of vertical ocean mixing efficiency on the tropical Atlantic in a coupled global climate model., under review at Climate Dynamics 


\section{Abstract}

Sea surface temperature (SST) biases in the tropical Atlantic are a long-standing problem among coupled global climate models (CGCMs). They occur in equilibrated state, as well as in initialised seasonal to decadal simulations. The bias is typically characterised by too high SST in upwelling regions and associated errors of wind and precipitation. We examine the SST bias in the state-of-the-art CGCM EC-Earth by means of an upper ocean heat budget analysis. Horizontal advection processes affect the SST bias development only to a small extent, and surface heat fluxes mostly dampen the warm bias. Subgrid-scale upper ocean vertical mixing is too low in EC-Earth when compared to estimates from reanalysis data, potentially giving rise to the warm bias. We perform sensitivity experiments to examine the effect of enhanced vertical mixing on the bias in quasi equilibrium present day climate and its impact on projected climate change. Enhanced mixing in historical simulation mode $\left(\mathrm{MixUp}_{p r}\right)$ reduces the SST bias in the tropical Atlantic compared to the control experiment $\left(\mathrm{Control}_{p r}\right)$. Associated atmospheric biases of precipitation and surface winds are also reduced in MixUp $p_{p r}$. We perform climate projections under the RCP8.5 emission scenario $\left(\mathrm{Control}_{f u}\right.$ and $\left.\mathrm{MixUp}_{f u}\right)$. Under increasing greenhouse gas forcing, the tropical Atlantic warms by up to $4.5^{\circ} \mathrm{C}$, and maritime precipitation increases. We show that the vertical mixing parameterisation asserts a distinct influence on the climate change signal. In MixUp $\mathrm{p}_{f u}$, SSTs remain $0.5^{\circ} \mathrm{C}$ colder in boreal winter and spring, but increase with the same amplitude in summer and fall. The strength and location of the projected intertropical convergence zone also depends on the ocean vertical mixing efficiency. The rain band moves northward in summer and fall and is increased in winter in $\mathrm{MixUp}_{f u}$ as compared to Control $_{f u}$. 


\subsection{Introduction}

The importance of the tropical Atlantic to climate variability is evident from the sizable impacts it asserts on the surrounding continents. Tropical Atlantic sea surface temperatures (SST) are related to precipitation over Africa (Rouault et al., 2003; Okumura and Xie, 2004), the Indian Summer Monsoon (Kucharski et al., 2009), as well as to drought and rainfall in the Brazilian Norderste region (Nobre and Shukla, 1996; Pezzi and Cavalcanti, 2001; Giannini et al., 2004; Yoon and Zeng, 2010). The tropical Atlantic modulates the North Atlantic Oscillation (NAO) (Okumura et al., 2001; Haarsma and Hazeleger, 2007), influences heat waves in Europe (Cassou et al., 2005) and precipitation in North America (Kushnir et al., 2010).

Yet, SST biases in the tropical Atlantic are a longstanding problem among state-of-the-art coupled global climate models (CGCMs). They commonly feature a large southeastern warm bias off the coast of Africa, which extends along the equator (Wang et al., 2014). The origins of this bias are not fully understood, even though the question has received considerable attention. See for example Wahl et al. (2011); Richter et al. (2012); Patricola et al. (2012); Exarchou et al. (2017); Koseki et al. (2018); Prodhomme et al. (2019), and references therein. Several studies point to westerly wind biases along with misrepresented Amazonian precipitation as its main cause (Wahl et al., 2011; Richter et al., 2012; Voldoire et al., 2014). Indeed, the important role of wind stress at the ocean surface has recently been underlined by Wen et al. (2017) and Voldoire et al. (2019). Enhancing the atmospheric model resolution can improve the simulation of the wind field and thereby the simulation of tropical Atlantic variabilty (Harlaß et al., 2018).

Ma et al. (1996b) link stratocumulus cloud cover to the cold tongue bias in the Pacific and Bellomo et al. (2015) show that local cloud feedbacks are equally important in the tropical Atlantic. Huang et al. (2007) and Hu et al. (2008) have linked low cloud cover to bias development in he NCEP coupled forecast system (CFS). Hourdin et al. (2015) point to the role of evaporation and near surface relative humidity, and $\mathrm{Hu}$ et al. (2011) note the importance of a correctly simulated cloud liquid water path. Whether deficient low cloud cover in the southeastern tropical Atlantic forces the bias or is a result thereof remains under discussion (Large and Danabasoglu, 2006; Xu et al., 2014a; Richter, 2015).

While atmospheric biases may partly explain the warm SST bias, the ocean model also contributes. Increased ocean resolution often leads to improvements in the tropical Atlantic (Seo et al., 2006; Doi et al., 2012; Small et al., 2014). However, part of the warm SST bias remains, even when forcing eddy-resolving ocean models with reanalysis wind stress (Xu et al., 2014b). The authors of the latter study discuss the influence of the biased equatorial thermocline on ocean dynamics. The position of the thermocline is connected to large parts of SST variability in the tropical Atlantic, for example via the Bjerknes Feedback, which causes the leading mode of inter-annual variability (Keenlyside 
and Latif, 2007; Deppenmeier et al., 2016), as well as contributing to the seasonal cycle (Burls et al., 2011). The important role of smaller scale ocean processes, especially of turbulent mixing, has been pointed out by Hazeleger and Haarsma (2005); Hummels et al. (2014), Polo et al. (2015), and Planton et al. (2018). In the tropical Pacific, insufficient vertical mixing has been proposed as a possible candidate for causing the warm biases in ocean models from observational analysis (Moum et al., 2013). It might play an important role in the tropical Atlantic, as well.

The development of biases in coupled climate models can be studied from ensembles of initialised hindcast simulations. For these experiments, the model components for the atmosphere and the ocean are initialised with estimates of the observed state, for example constructed from reanalysis data. Choosing an initialisation date in the past allows one to study the development of the bias by tracing the simulation's deviations from observations (Toniazzo and Woolnough, 2014; Huang et al., 2007; Vannière et al., 2013; Gaetani and Mohino, 2013; Voldoire et al., 2019). Assuming the initialisation shock is small (Balmaseda and Anderson, 2009), possible feedbacks leading to the biases can be followed and their origins untangled. This is not possible with data from an equilibrated climate model simulation.

In this study, we use initialized seasonal hindcasts to study the origin of the warm bias in the tropical Atlantic by means of a closed heat budget analysis. From this analysis, we form a hypothesis that places upper ocean vertical mixing at the center of the warm bias development. To test this hypothesis we perform a sensitivity experiment with altered parameterizations initialized from equilibrated biased model state, as well as a control experiment with the unchanged version of the climate model EC-Earth 3.2.3 (climate mode simulations). We continue the experiments into the 21 st century to analyse the impact of enhanced mixing on the tropical Atlantic climate change signal due to rising greenhouse gases.

The data used in this study is described in Section 4.2. The results are presented in Section 4.3, first from the seasonal hindcasts (Section 4.3.2) and then the sensitivity experiments (Section 4.3.3), and placed in context in Section 4.4.

\subsection{Data and Methodology}

To identify the origin of the SST bias, we construct an upper ocean heat budget from an ensemble of seasonal hindcasts. Each seasonal hindcast is initialised from an estimate of the observed state constructed from reanalysis data on the first of May between 20002009. The reanalysis products chosen for the initialisation are based on the ocean and atmosphere models native to EC-Earth, ORAS4 (Balmaseda et al., 2013) (NEMO), and ERA-Interim (Dee et al., 2011) (IFS). The hindcasts are integrated over four months, 
from the 1st of May until the 31st of August. We focus on boreal summer, when the tropical Atlantic cold tongue develops, and model biases grow concurrently. Repeating the experiment four times starting form perturbed atmospheric initial states leads to an ensemble of five members for each year. The ensemble is generated with EC-Earth version 3.2.1. The model is based on EC-Earth2.2 (Hazeleger et al., 2010, 2012) with updated atmosphere, ocean, sea ice (modeled by LIM3 (Vancoppenolle et al., 2008)), and aerosol components. It consists of the NEMO ocean engine version 3.3 using the ORCA1 grid and 46 vertical levels (ORCA1L46) (Madec et al., 2011) and IFS cycle 36r4 with triangular truncation at truncation at wavenumber 255 and 91 vertical levels up to $5 \mathrm{hPa}$ (T255L91, Riddaway, Newsletter ECMWF 2010). The individual components are coupled via OASIS3 (Valcke, 2013).

Surface heat and radiative fluxes from TropFlux (Kumar et al., 2012), wind stress from ERA-Interim (Dee et al., 2011) and the ECMWF ocean reanalysis system 4 (ORAS4, (Balmaseda et al., 2013)) are used to construct a reanalysis upper ocean heat budget.

Heat budgets are calculated for two regions in which the SST bias is large in the seasonal hindcasts as well as in the equilibrated control experiment (Fig. 4.1 and Fig. 4.2): off the Angolan-Namibian coast, (AN regionbox, $4^{\circ} \mathrm{E}-11^{\circ} \mathrm{E}, 6^{\circ} \mathrm{S}-18^{\circ} \mathrm{S}$ ), and underneath the ITCZ (ITCZ box, $25^{\circ} \mathrm{W}-8^{\circ} \mathrm{W}, 2^{\circ} \mathrm{N}-5^{\circ} \mathrm{N}$ ). They are indicated by the boxes in Fig. 4.2. The differences between the model and reanalysis budgets are used to study the development of the SST bias.

Seasonal hindcasts are costly, therefore we use comparatively cheap climate mode simulations to test our hypotheses in an equilibrated situation. For the climate mode simulations we use the CMIP6 version of EC-Earth3, consisting of LIM3, NEMO3.6 with the ORCA1 grid with 75 vertical levels, and IFS cycle 36r4 with T255L91. The resolution in the atmosphere and the horizontal resolution of the ocean are identical to the one in the seasonal hindcasts, but the ocean model in EC-Earth3 features more vertical levels. We generate one member for each sensitivity experiment. The historical simulations Control $_{p r}$ and MixUp $p_{p r}$ start from spun up conditions in 1950 and are forced with CMIP5 historical forcing (1950-2010). The climate projections Control $_{f u}$ and $\mathrm{MixUp}_{f u}$ under RCP8.5 forcing (Riahi et al., 2011) start in 2010 from the simulated states of Control pr and $\mathrm{MixUp}_{p r}$. These experiments are continued until the end of the century (2099). For evaluation of future climate we use the period between 2070-2099, for evaluation of the present climate we use data from 1979-2009. The present climate period begins in line with the availability of reliable reanalysis data, and ends before RCP8.5 forcing is applied. The setup of the MixUp experiments is explained in more detail in Section 4.3.3, and the experiments are listed in Table 4.1. 
Table 4.1: Sensitivity experiments conducted for this study. All simulations are performed with an atmospheric component with triangular spectral truncation at wavenumber 255 with 91 vertical levels. Only differences between the experiments are mentioned.

\begin{tabular}{l|cccc} 
Experiment & Ocean resolution & $C_{d i f f}$ & Start date & End date \\
\hline Seasonal hindcasts & ORCA1L46 & 0.1 & May 1st 2000-2009 & Aug 31st 2000-2009 \\
Control $_{p r}$ & ORCA1L75 & 0.1 & Jan 1st 1950 & Dec 31st 2009 \\
MixUp $_{p r}$ & ORCA1L75 & 0.5 & Jan 1st 1950 & Dec 31st 2009 \\
Control $_{f u}$ & ORCA1L75 & 0.1 & Jan 1st 2010 & Dec 31st 2099 \\
MixUp $_{f u}$ & ORCA1L75 & 0.5 & Jan 1st 2010 & Dec 31st 2099
\end{tabular}

\subsection{Results}

\subsubsection{Biases in the historical simulation and in the seasonal hindcasts}

The annual tropical Atlantic SST bias in EC-Earth closely resembles the bias of other CMIP5 models (Taylor et al., 2012) in equilibrium state (Wang et al., 2014, Fig. 1a). It extends from the western coast of Africa into the tropical Atlantic Ocean, close to the equator (Fig. 4.1a). In May, June, July, and August, when the annual equatorial cold tongue develops and is present in observations, the bias is even larger (Fig. 4.1b).

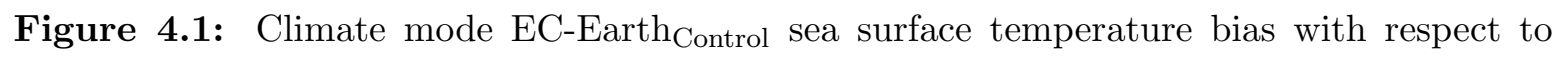
ERA-Interim in present climate for the period of 1979-2009. Annually averaged bias in panel $\mathrm{a}$, and the heightened summer time bias in the months May, June, July, August in panel b.

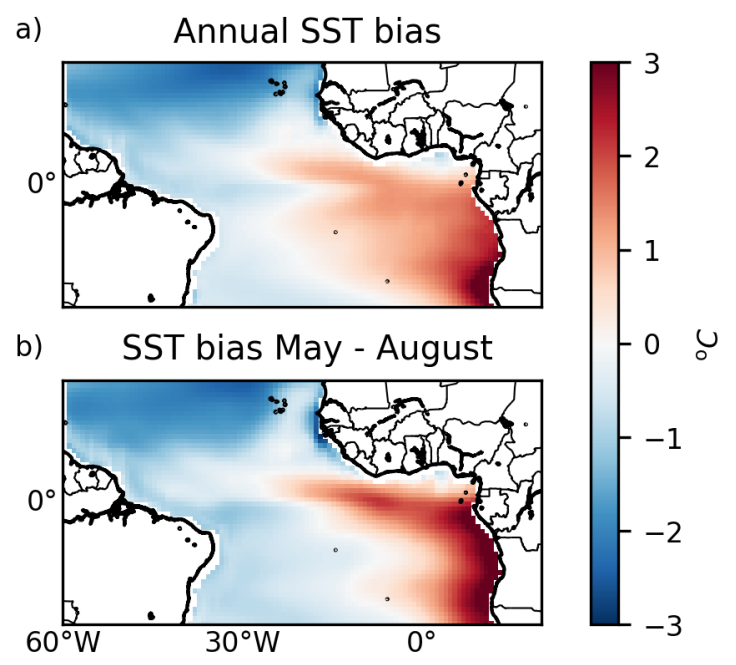

The CGCM is not able to produce sufficiently strong cooling during boreal summer and displays a bias with root mean square errors on the order of 1 to $2 \mathrm{~K}$ in the ITCZ and AN boxes (Table 4.2), locally the biases are even larger. While these biases are smaller 
than those of other CGCMs (Toniazzo and Woolnough, 2014), their spatial pattern is very similar. Since the atmosphere and ocean in the tropics are strongly coupled, CGCM biases in one component affect the wider climate system. Therefore, we examine the seasonally stratified biases of SST, wind and precipitation in the control simulation.

Table 4.2: SST root mean square distance between EC-Earth experiments (Control and MixUp) and ERA-Interim (ERA), and between EC-Earth MixUp $_{\text {and EC-Earth }}$ Control.

\begin{tabular}{l|ccc|ccc|ccc} 
& \multicolumn{3}{|c|}{ Control - ERA } & \multicolumn{3}{c|}{ MixUp - ERA } & \multicolumn{3}{c}{ Control - MixUp } \\
\hline Present & AN & ITCZ & ATL3 & AN & ITCZ & ATL3 & AN & ITCZ & ATL3 \\
\hline DJF & 2.23 & 0.79 & 0.92 & 1.57 & 0.75 & 0.90 & 0.66 & 0.04 & 0.01 \\
MAM & 2.14 & 0.59 & 0.78 & 1.22 & 0.45 & 0.19 & 0.92 & 0.15 & 0.60 \\
JJA & 2.42 & 0.85 & 1.38 & 2.32 & 0.85 & 1.36 & 0.10 & 0.00 & 0.02 \\
SON & 2.31 & 0.81 & 0.79 & 2.13 & 0.86 & 0.97 & 0.17 & 0.05 & 0.18 \\
YRL & 2.24 & 0.73 & 0.93 & 1.73 & 0.71 & 0.80 & 0.51 & 0.02 & 0.13 \\
\hline Future & & & & & & & AN & ITCZ & ATL3 \\
\hline DJF & & & & & & & 0.35 & 0.06 & 0.09 \\
MAM & & & & & & & 0.68 & 0.26 & 0.36 \\
JJA & & & & & & & 0.14 & 0.29 & 0.25 \\
SON & & & & & & & 0.03 & 0.38 & 0.34 \\
YRL & & & & & & & 0.27 & 0.08 & 0.06
\end{tabular}

Figure 4.2: Fast developing seasonal hindcast sea surface temperature bias in EC-Earth after three months of runtime. The start date of the seasonal hindcasts is the 1st of May every year between 2000 and 2009. The bias is calculated with respect to ERA-Interim data from 2000-2009.

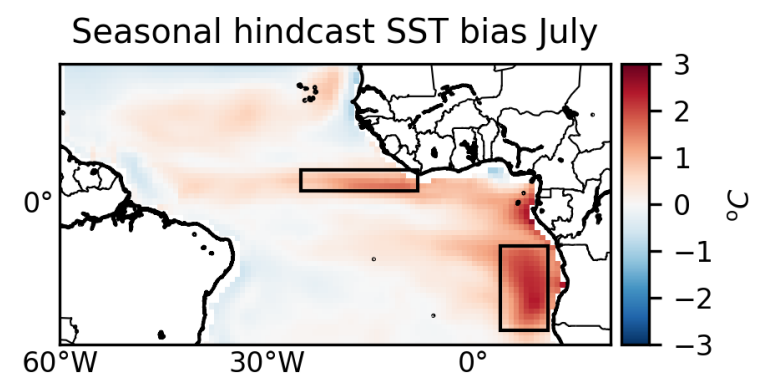

Throughout all seasons, south-eastern tropical Atlantic SSTs are too warm (Fig. 4.4), in line with Figs. 4.1 and 4.2. The maximum SST bias moves northward towards the cold tongue region along the equator from boreal winter to summer. Its extent along the equator is greatest in spring, albeit with a smaller amplitude than in winter and summer.

The SST bias has a meridional dipole structure and the location of the ITCZ is associated by with this gradient (Biasutti et al., 2003, 2006). As a consequence, the ITCZ is 
The effect of vertical ocean mixing efficiency on the tropical Atlantic in a 78 coupled global climate model.

Figure 4.3: Seasonal cycle of sea surface temperature in colours, wind vectors, and precip-

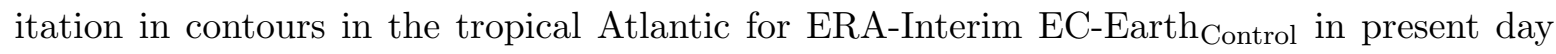
climate (1979-2009).
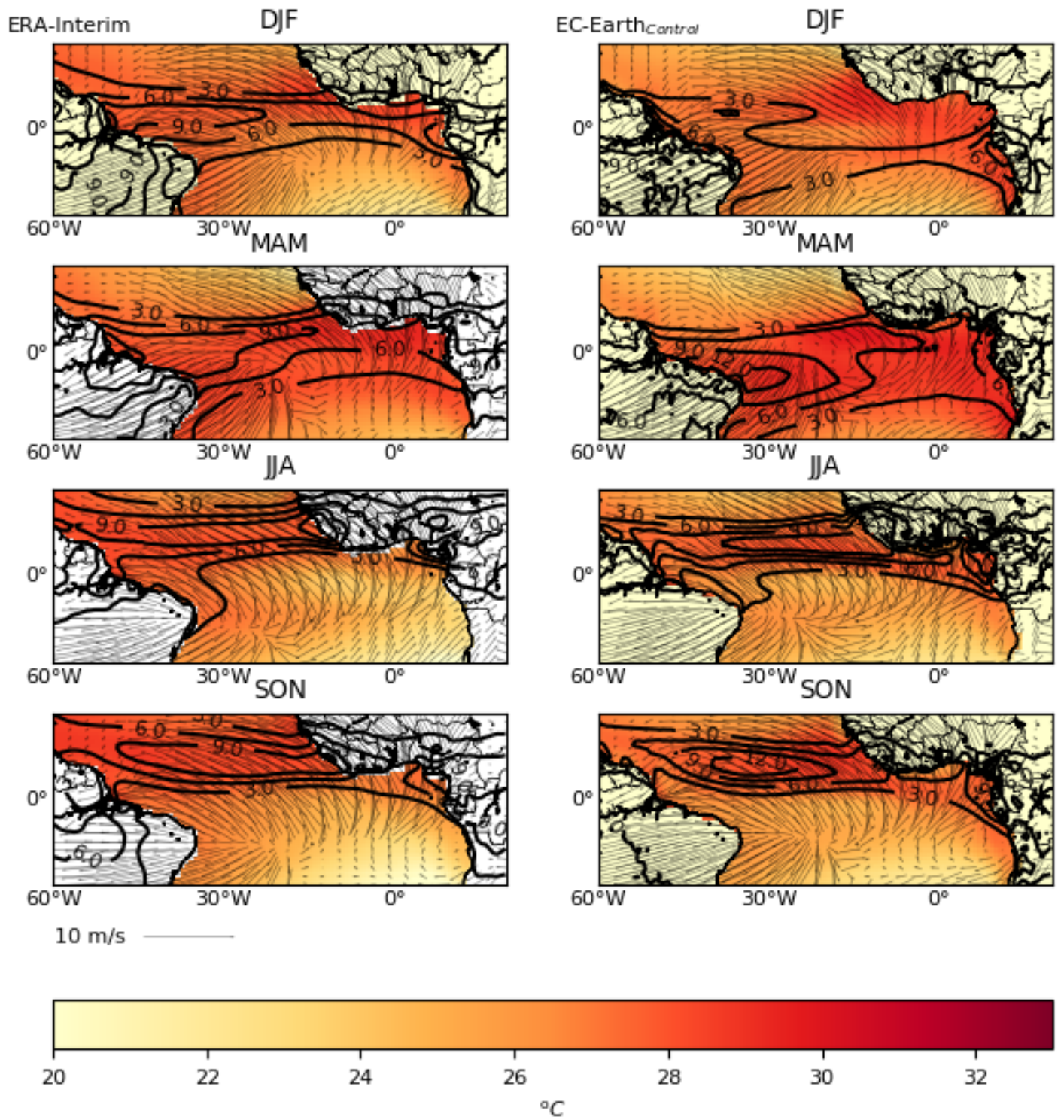

located too far south in EC-Earth compared to reanalysis data. EC-Earth underestimates accumulated rainfall underneath the ITCZ in all seasons (Fig. 4.4).

Coupled to the ITCZ bias, the surface wind bias also follows the meridional SST bias with southward anomalies across the equator. Surface winds in the eastern equatorial Atlantic (ATL3 box, $25^{\circ} \mathrm{W}-0^{\circ} \mathrm{E}, 3^{\circ} \mathrm{N}-{ }^{\circ} \mathrm{S}$ ) are underestimated by more than $50 \%$ in boreal winter, and by about $40 \%$ in spring. The bias decreases in early summer and switches sign from July to September. The overestimation only reaches $15 \%$, so that the annual average bias is negative. In the southeast, surface winds are overestimated by up to $20 \%$ from June 
Figure 4.4: Seasonal cycle of sea surface temperature bias in colours, wind bias in vectors, and precipitation bias in contours in the tropical Atlantic as in Fig. 4.3, but for the difference

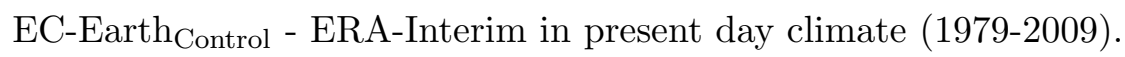

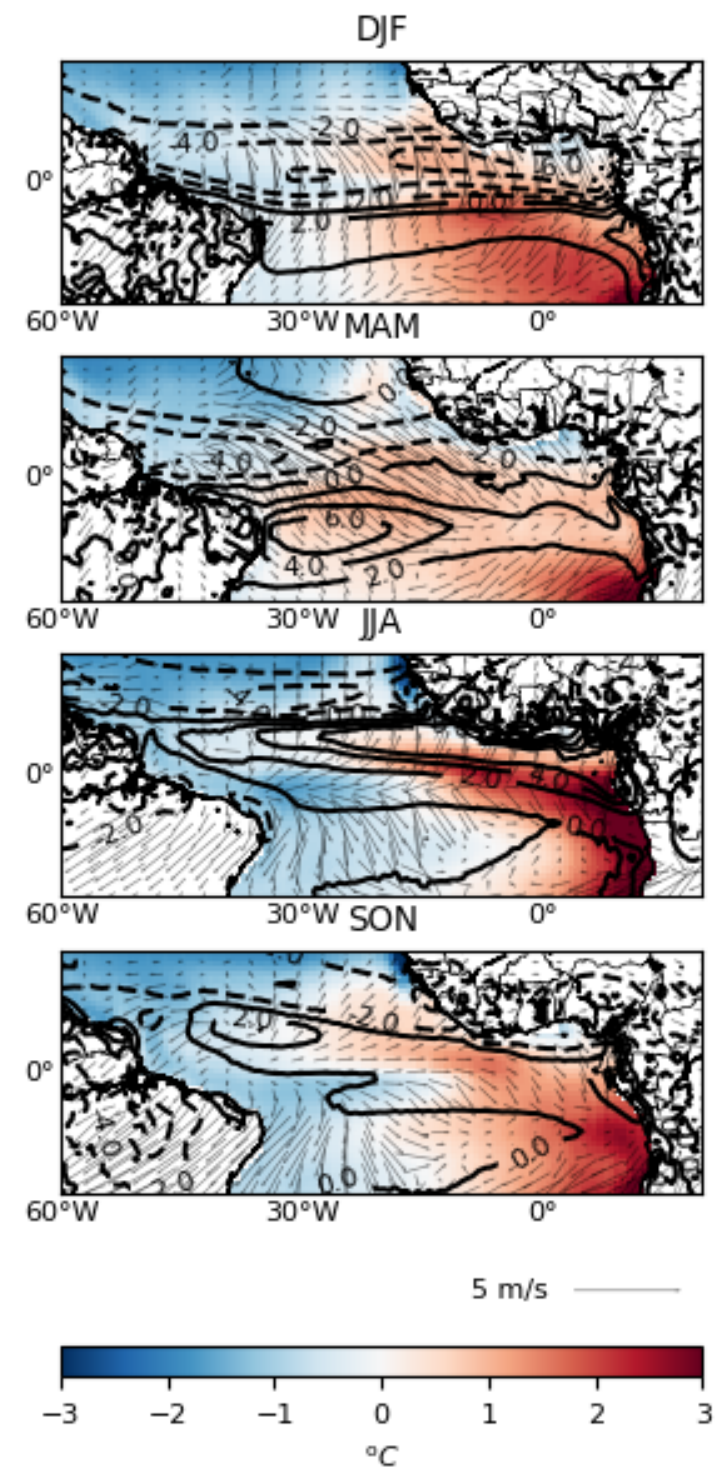

until September, and underestimated by up to the same amount from October to April. In the western equatorial Atlantic surface winds are well reproduced during most of the year, but are underestimated by more than $40 \%$ in spring. The bias peaks in May.

EC-Earth performs better than most climate models in this region. For instance, it does not exhibit the notorious double ITCZ structure many other models suffer from (Huang et al., 2004; Biasutti et al., 2006; Deser et al., 2006; Breugem et al., 2006, 2007; Lin, 2007), although it produces rainfall too far to the south of observed values (see Fig. 4.3).

We study the origin of the warm SST bias, and potentially the atmospheric biases that 
accompany it, with an upper ocean heat budget analysis on the seasonal hindcast data set. Only three months after initialisation, i.e. in July, a bias pattern emerges that is very similar to the equilibrium bias (Fig. 4.2). The amplitude is weaker, likely due to slow adjustment time scale of the ocean, but we can assume that the physical mechanisms responsible for the fast developing bias also contribute to the equilibrated bias.

\subsubsection{Upper ocean heat budget}

Two heat budgets are calculated, one from reanalysis and one from model output, and subtracted from each other to form the bias development budget. The upper ocean heat budgets are constructed according to the following equation

$$
\partial_{t} T_{s}=\frac{Q}{h \rho_{w} c_{p}}-w \partial_{m x l} T-u \partial_{x} T-v \partial_{y} T+R
$$

In Eq. 4.1, $\partial_{t} T_{s}$ is the temperature evolution in the upper mixed layer approximated by the SST evolution, $Q$ is the net surface flux into the mixed layer, $u, v$, and $w$ are the horizontal and vertical current velocities ( $w$ is calculated from the horizontal divergence of $u$ and $v) . \rho_{w}$ is the density of seawater, $c_{p}$ its heat capacity, and $h$ is the mixed layer depth according to a temperature threshold $(T \leq S S T-0.1)$. This compares well to the mixed layer depth obtained from a density threshold (not shown). $R$ is the residual comprising all subgrid-scale terms.

We calculate the difference between the reanalysis and the model budgets for the two boxes shown in Fig. 4.2. In the AN box, the SST bias rises monotonously throughout the first two months of the simulation (Fig. 4.5a). Excess incoming shortwave radiation has been named as a possible explanation of the bias (Huang et al., 2007; Hu et al., 2008). In our case, the net surface fluxes immediately dampen the bias. A positive shortwave radiation bias eventually develops, but only after two weeks into the simulation (not shown). This is after an initial SST bias has already been formed.

The heat budget shows that, in the first two months, mean upwelling contributes little to the bias. Horizontal advection does not contribute to the fast response bias, either. The subgrid-scale processes, captured in the residual, are the only large positive contribution to the SST bias. This term encompasses all processes that are not explicitly solved in the numeric climate model. These processes consist of vertical mixing, diffusion, mesoscale eddies, and horizontal turbulent processes. Among those terms, turbulent vertical mixing is likely the most important one. Observations show that horizontal and vertical diffusion are both smaller by an order of magnitude (Foltz et al., 2003), and lateral subduction is expected to be small in regions with a horizontally homogeneous mixed layer depth such as in the tropics. In regions where tropical instability waves (TIWs) are present the mesoscale eddy transports can be sizable (Hazeleger et al., 2001), but these regions are 
Figure 4.5: Sea surface temperature bias development and the contributions to it in the ECEarth seasonal hindcasts. Panels a and b show the time integrated contributions to the sea surface temperature bias evolution according to the upper ocean heat budget (Equation 4.1), for the AN and the ITCZ box respectively. The individual components contributing to the warm bias are mean horizontal advection (UV), mean vertical advection (W), the net heat flux from the surface into the well mixed layer (Q), and subgrid-scale and short timescale processes that are parameterised in the model and not available from reanalysis data $(\mathrm{R})$. We refer to section 4.3.2 for the methodology and data used to calculate the heat budget. Panels $\mathrm{c}$ and $\mathrm{d}$ show the seasonal hindcast subsurface temperature bias development in the two boxes with respect to ORAS4.
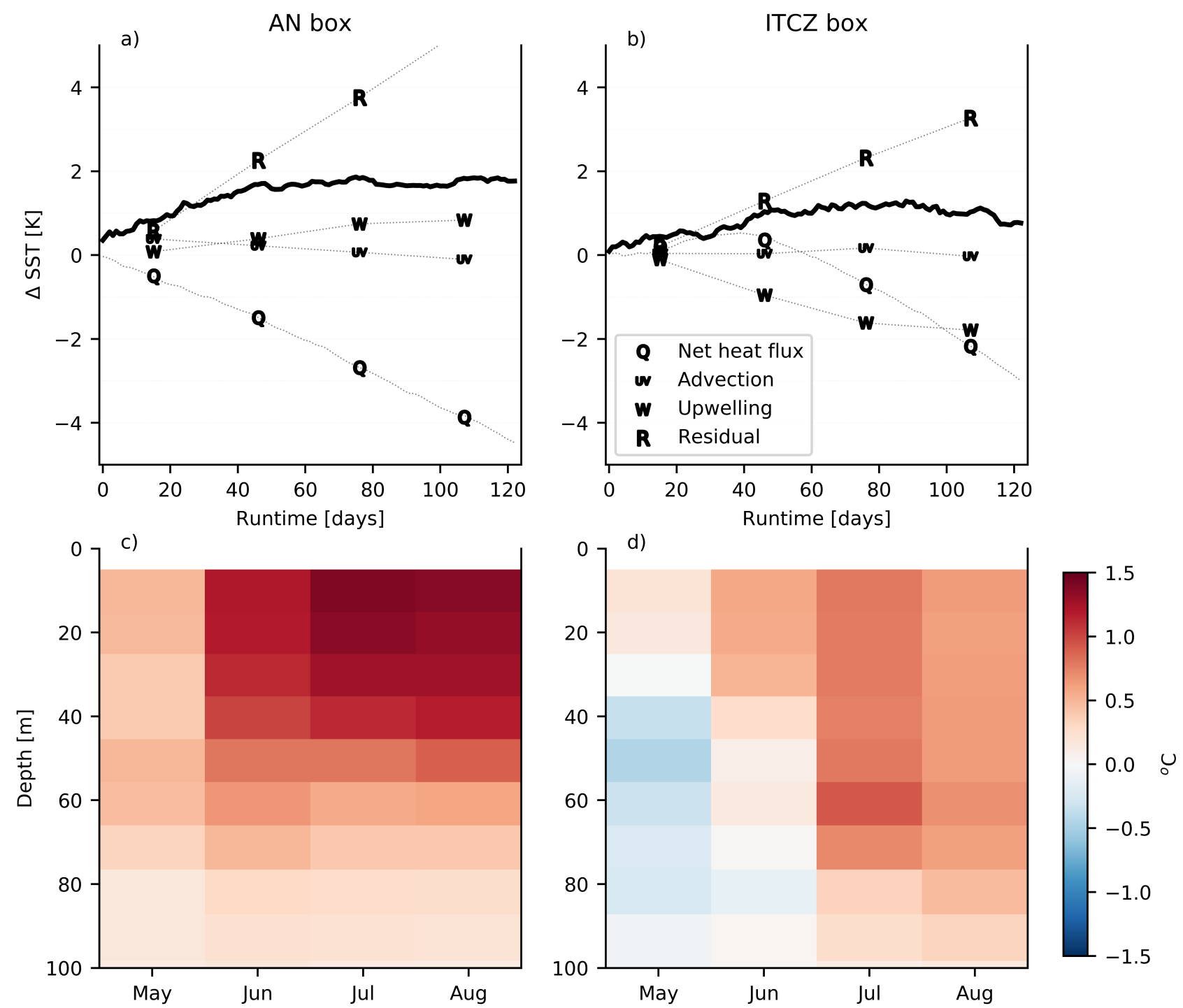

closely confined to the equatorial region and likely do not penetrate the southeastern part of the tropical Atlantic (Jochum et al., 2004). 
The ocean column in the AN region also shows approximately monotonous warm bias development (Fig. 4.5c). A small bias throughout the ocean column develops throughout the first month. The bias grows in the well mixed surface layer, down to $20 \mathrm{~m}$ to $40 \mathrm{~m}$. In July it reaches its maximum at the surface, and in August it spreads through the upper $50 \mathrm{~m}$.

The SST bias in the ITCZ region develops in two stages. In the first month after initialisation, the bias is smaller than $0.5{ }^{\circ} \mathrm{C}$. It seems to be formed by excess net surface heat fluxes (Q) in June. Later on, Q becomes negative, and dampens the bias. After the initial onset of the bias by $\mathrm{Q}$, the residual becomes the sole large positive contribution to the SST bias, similar to the development in the AN box. In the absence of large positive contributions of surface flux heating, this implies that one or more subgrid-scale processes cooling the ocean surface are weaker in the CGCM than in ORAS4. Underneath the ITCZ we can expect an impact of mesoscale and smaller scale processes (e.g. TIWs), but vertical mixing is expected to play a more prominent role (Foltz et al., 2003).

The upper ocean heat budget analyses for both AN and ITCZ suggest that subgridscale processes play a dominant role in the bias development in the seasonal hindcast simulations. We hypothesise that vertical mixing is the most important among these processes, and that it is underestimated in coupled global climate model simulations.

\subsubsection{Sensitivity experiments}

\section{Setup of the enhanced vertical mixing experiments MixUp}

Turbulent motion acts on such small time and length scales that it cannot be resolved in global climate models, and hence has to be parameterized. Therefore, turbulent coefficients are defined in analogy to molecular diffusion and viscosity which act on the gradients of temperature, salt and momentum to mimic turbulent motion.

The vertical eddy diffusivity coefficient $A_{v t}$ acts on the temperature field according to the diffusive operator $D_{v t}$ :

$$
D_{v t}=\frac{\partial}{\partial z}\left(A_{v t} \frac{\partial T}{\partial z}\right)
$$

The temperature $T$ and the height of the layer $z$ along the vertical index $k$ are resolved in the model, the vertical eddy diffusivity coefficient $A_{v t}$ needs to be prescribed or calculated from resolved variables.

In the ocean component of EC-Earth, NEMO (Madec et al., 2015), several methods are available to compute the vertical diffusivity coefficients. It can be chosen as constant, dependent on the local Richardson number, or calculated with a turbulence closure scheme. In this study we use the turbulent kinetic energy (TKE) scheme. This scheme was first introduced by Bougeault and Lacarrere (1989) in the atmosphere, adapted for use in the 
ocean by Gaspar et al. (1990), and introduced into OPA, the former version if NEMO, by Blanke and Delecluse (1993). It has been adapted and extended by Madec et al. (1988). In the following, we describe the employed TKE scheme following the description of the NEMO documentation (Madec et al., 2015).

The TKE scheme employs the prognostic equation for the turbulent kinetic energy $\bar{e}$ :

$$
\begin{aligned}
\frac{\partial \bar{e}}{\partial t}=\frac{A_{v m}}{h^{2}}\left[\left(\frac{\partial u^{2}}{\partial z}\right)+\left(\frac{\partial v^{2}}{\partial z}\right)\right]-A_{v t} N^{2}+\frac{1}{h} \frac{\partial}{\partial z}\left[\frac{A_{v m}}{h} \frac{\partial \bar{e}}{\partial z}\right]-c_{\epsilon} \frac{\bar{e}^{\frac{3}{2}}}{l_{\epsilon}} \\
A_{v m}=C_{d i f f} \cdot l_{m x l} \cdot \sqrt{\bar{e}} \\
A_{v t}=A_{v m} / P_{r t}
\end{aligned}
$$

In Equation $4.3 t$ is time, $h$ is the depth of the ocean layer, defined in the vertical along $z$, $u$ and $v$ are the meridional velocities. In all cases in this study, the Prandtl number, set as a function of the Richardson number $R i\left(P_{r t}=1\right.$ for $\left.R i \leq 0.2\right)$ is $P_{r t}=1$, and hence $A_{v t}=A_{v m} . l_{m x l}$ in Equation 4.4 is the mixing length scale. Both $l_{\epsilon}$ and $l_{m x l}$ are dependent on the square root of the turbulent kinetic energy and the Brünt-Väisälä frequency

$$
\begin{aligned}
l_{\epsilon} & =l_{m x l}=\sqrt{2 \bar{e}} / N \\
N^{2} & =-\frac{g}{\Theta} \frac{\partial \Theta}{\partial z}
\end{aligned}
$$

Note that $N^{2}$ is calculated from temperature $T$ and salinity $S$ and thermal and haline expansion coefficients $\alpha$ and $\beta$ which are functions of $T$ and $S . l_{\epsilon}$ and $l_{m x l}$ are additionally bound by physical reasoning about the vertical length scale they can achieve. In the surface layer this scaling takes into consideration the penetration depth of turbulent kinetic energy due to wind stress forcing.

In Equation 4.3 the first term on the right hand side expressed turbulence production due to vertical shear, the second term is the destruction of turbulence due to stratification, the third term represents the vertical diffusion of turbulent kinetic energy, and the last term its Kolmogorov dissipation (Kolmogorov, 1941).

Apart from the turbulence generation by vertical shear, Langmuir cells (LC) and internal wave breaking (IWB) give rise to turbulent kinetic energy, and at the surface TKE is injected into the ocean column by the magnitude of the local wind stress $|\tau|$

$$
\bar{e}=3.75 \frac{|\tau|}{\rho_{0}},
$$

where $\rho_{0}$ is the reference density defined as $1,035 \mathrm{~kg} / \mathrm{m}^{3}$. For more detail on the implementation of the parameterization schemes we refer to Madec et al. (2015). 
In this study we test the effect of enhanced vertical mixing on the tropical Atlantic. In the most straightforward manner, this can be achieved by increasing $A_{v t}$. To keep the model's physical consistency and allow the mixing coefficient to react to the available turbulence kinetic energy, we do not prescribe $A_{v t}$, but instead allow the model to calculate the coefficient according to its algorithms.

Testing different parameters in 3D setting is costly. Instead we performed a number of sensitivity experiments with a coupled single column version of EC-Earth, to determine which parameter changes should be made to enhance vertical mixing in the ocean (Deppenmeier et al., under review at Journal of Climate). Based on these experiments, we increase $C_{\text {diff }}$ in Equation 4.4, which determines the turbulent eddy diffusivity coefficient, from the reference value of 0.1 to 0.5 . $C_{\text {diff }}$ is constrained by the mixing efficiency $\gamma$, according to

$$
C_{d i f f}=\frac{1}{2} \cdot \gamma \cdot P_{r t} * C_{\epsilon} .
$$

Observational estimates for $\gamma$ differ (Gaspar et al., 1990, and references therein). The value chosen for this study is larger than suggested by the measurements, which allow for $C_{\text {diff }}=0.3$. We chose to enhance $C_{\text {diff }}$ drastically to study the effect of enhanced mixing and reduced warm surface layer formation.

The increase of $C_{\text {diff }}$ can be understood as an enhanced model efficiency to use the available turbulent kinetic energy to mix the ocean column. We maintain the physical reasoning behind the TKE calculation, while strongly strengthening the mixing capability.

Following our hypothesis derived from the heat budget analysis on initialised seasonal hindcasts, we increase $C_{d i f f}$ in sensitivity experiments (MixUp). These model simulations are first performed in historical climate mode, i.e. with prescribed historical estimates of greenhouse gas and aerosol concentrations as well as land use as described in Data and Methodology section. Secondly, we perform future climate projections using RCP 8.5 forcing to investigate the impact on climate change projections of the tropical Atlantic region. For both historical and future simulations we perform control simulations with unchanged parameterisation, $\mathrm{Control}_{p r}$ and $\mathrm{Control}_{f u}$, and the corresponding sensitivity experiments MixUppr and MixUpfu.

\section{Impact of enhanced vertical mixing on present day climate}

The amplification of vertical heat and momentum exchange in the ocean column driven by turbulent kinetic energy successfully reduces the present day SST bias, both annually and in MJJA (Fig. 4.6).

Along the equator, between $3^{\circ} \mathrm{N}$ and $3^{\circ} \mathrm{S}$, the warm bias is reduced in $\mathrm{MixUp}$ pr , especially the early spring (Figs. 4.6 and 4.7). Between February and April, Control $_{p r}$ features an 
Figure 4.6: Panel a shows the sea surface temperature bias in EC-Earth $\mathrm{MixUp}_{\mathrm{p}}$ with respect to ERA-Interim for the period May - August in present climate (1979-2009). Panel b shows the difference between the sensitivity experiment EC-Earth MixUp $_{p}$ and the control experiment in the same period. Note the different scales of the colorbars.
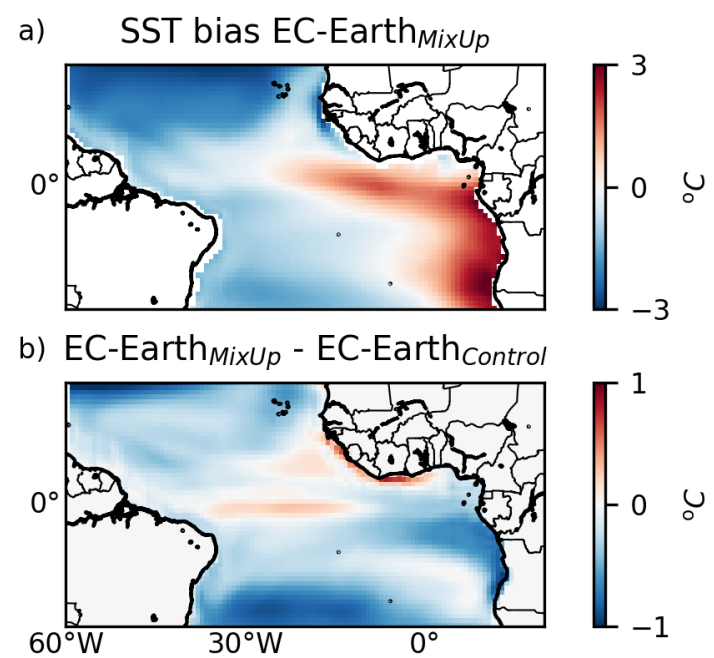

artificial central Atlantic warm pool, which is largely eliminated in MixUppr (Fig 4.7, top row). In boreal summer, when the cold tongue extends along the equator in observations, the control simulation misplaces the center of the cold water to the west and underestimates the strength of the cooling. While this leads to a warm bias in the cold region, the westerward extension of the simulated cold tongue causes a cold bias in the western part of the basin (Fig. 4.7, bottom row). Both biases are reduced in MixUppr. Similarly, the strong bias close to the coast of Africa is reduced. The seasonality of the SST evolution along the equator is well captured in EC-Earth compared to other coupled models (Deppenmeier et al., 2016; Voldoire et al., 2019; Prodhomme et al., 2019), and is not affected by the changed parameterization.

\section{Subsurface ocean changes}

The enhanced ocean vertical mixing efficiency impacts temperatures at the sea surface, and also below the surface. In the AN box, the upper $25 \mathrm{~m}$ are cooler in MixUP ${ }_{p r}$ than in Control $_{p r}$ throughout the year (Fig. 4.8a). The cooling is stronger in boreal winter and spring than in summer and autumn. Below the first $25 \mathrm{~m}$, the subsurface ocean warms, displaying a maximum warming signal between 30 and $60 \mathrm{~m}$.

The turbulent vertical mixing coefficient $\mathrm{A}_{v t}$ is increased strongly in the upper 50 meters in MixUp $p_{p r}$ with respect to Control $_{p r}$ (Fig. 4.8b). In boreal summer the enhanced mixing extends down to the upper $100 \mathrm{~m}$ of the ocean column, which is deeper than in winter, when the changes are confined to the upper $50 \mathrm{~m}$. 
Figure 4.7: Monthly stratified sea surface temperature along the equator $\left(3^{o} \mathrm{~N}-3^{o} \mathrm{~S}\right)$ in present climate (1979-2009) for a) ERA-Interim, b) EC-Earth Control, and c) EC-Earth $_{\text {MixUp. }}$.

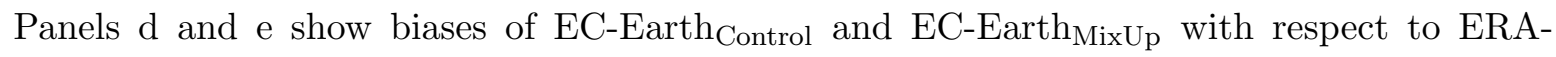
Interim.
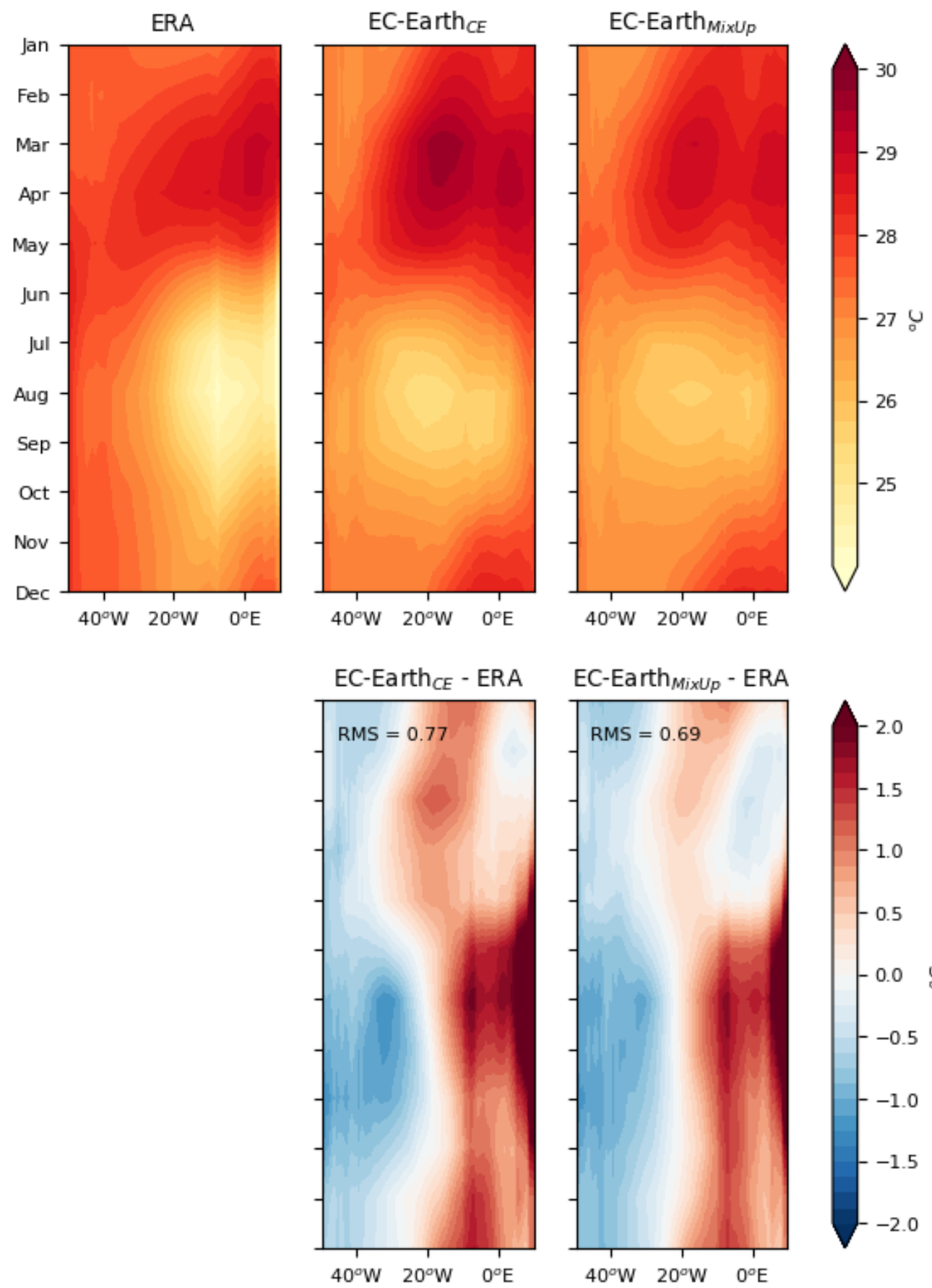

Enhanced vertical mixing in the upper $100 \mathrm{~m}$ explains the surface cooling, as well as the warming directly underneath the cooler layer. Heat is redistributed vertically in the well 
Figure 4.8: Difference between the $\mathrm{MixUp}_{p r}$ and Control $_{p r}$ in the upper ocean column in AN. The left panel depicts the box average change in temperature for each month of the year, the center panel the amplification of the vertical eddy diffusivity parameter $A_{v t}$. The right hand panel shows temperature difference throughout the year with depth in colours, and the isopycnals in contours $\left[1000-\mathrm{x} \mathrm{kg} / \mathrm{m}^{3}\right]$. The solid lines are the isopycnals in the control experiment, the dashed lines the isopycnals in the sensitivity experiment.

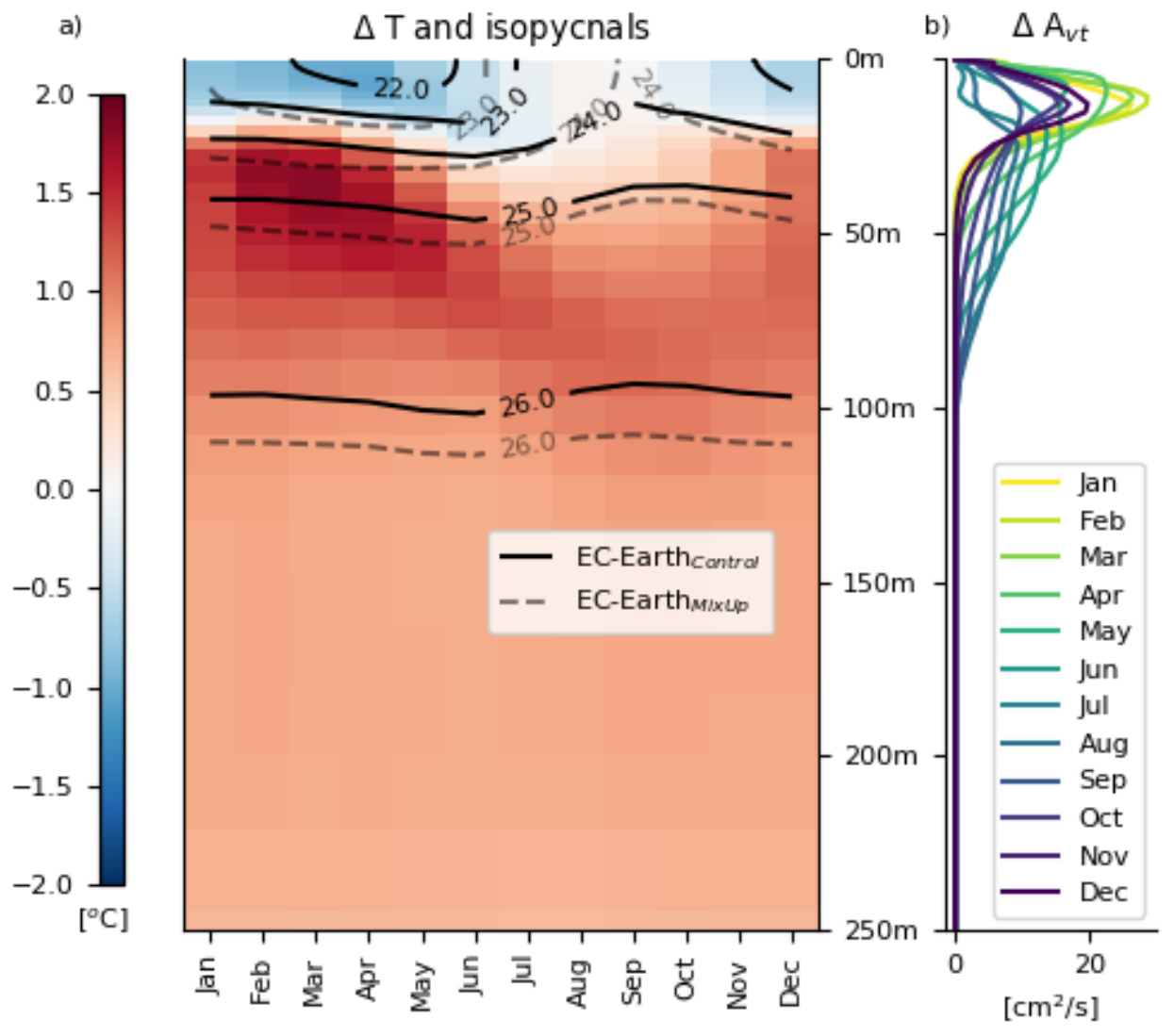

mixed layer, transported away from the surface, and then accumulates at the bottom of the well mixed layer. From here it cannot directly be distributed deeper into the column by fast mixing processes, due to the barrier of the thermocline. The mixing coefficient $\mathrm{A}_{v t}$ decreases rapidly (not shown). Hence, a dipole structure develops, with cooler water in the upper and warmer water in the lower part of the well mixed layer.

Another possible contribution to the subsurface warming is horizontal advection of warmer water along isopycnals. This can occur when the location where the density surface outcrops moves toward warmer surface waters, or by a change in subsurface currents. In $\mathrm{MixUp}_{p r}$, as compared to $\mathrm{Control}_{p r}$, Temperatures at the $1025 \mathrm{~kg} / \mathrm{m}^{3}$ isopycnal surface change slightly in the vicinity of the AN box (Fig. 4.9a). We choose this isopycnal because of its proximity to the subsurface warm bias. The changes are larger in the southern subtropical Atlantic and to the west of the AN box, but part of the subsurface warming 
Figure 4.9: Differences of a) ocean temperature along the $1025 \mathrm{~kg} / \mathrm{m}^{3}$ isopycnal and b) change of depth at wich that isopycnal is found between EC-Earth Control $_{\text {and EC-Earth }}$ ixUp. The sensitivity experiment is subtracted from the control experiment, so that negative (red) values show warming (deepening of the isopycnal) of the sensitivity experiment in comparison with the control. The $1025 \mathrm{~kg} / \mathrm{m}^{3}$ isopycnal is chosen for its location at the center of the warming signal between the two experiments (Fig. 4.8 and 4.11).
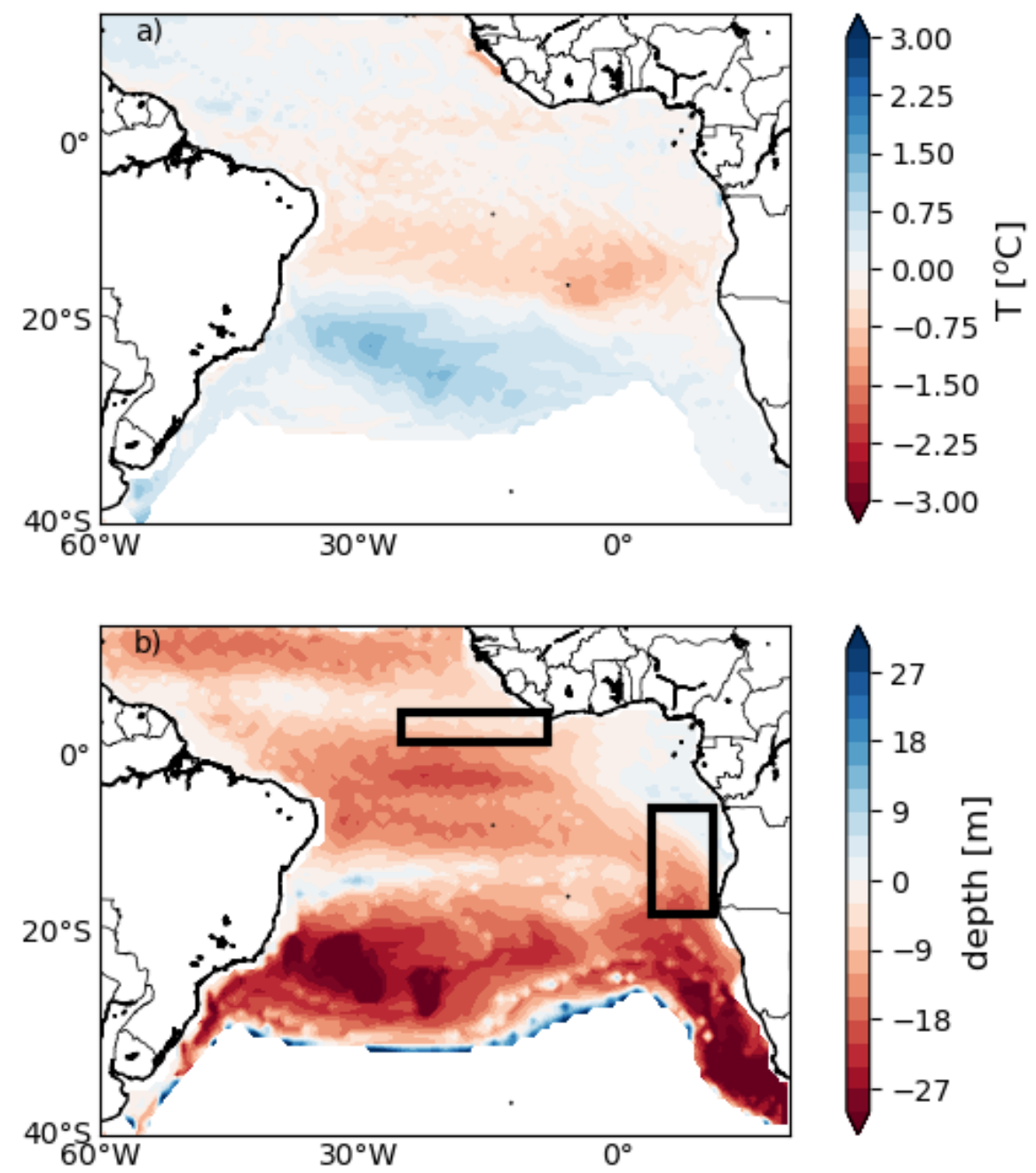

signal can be caused by warm water advected along the isopycnal into the AN box.

Furthermore, the $1025 \mathrm{~kg} / \mathrm{m}^{3}$ isopycnal is deeper in $\mathrm{MixUp}_{p r}$ than in Control $_{p r}$ (Fig. 4.9b, see also Fig. 4.8). A small change in the position of the isopycnals by shoaling leads to a large (warming) signal when it coincides with the steep gradient of the thermocline (Fig. 4.10). It is likely that remote as well as local processes play a role in the warm subsurface signal.

From the bottom of the well mixed layer, where the additional heat has accumulated, diffusion allows heat to penetrate into the deeper ocean on long timescales. The minimum 
Figure 4.10: Seasonally stratified ocean temperature with depth profiles of the upper $250 \mathrm{~m}$ in the AN and the ITCZ box, present climate (1979-2009). The green line shows ORAS4 reanalysis data, the orange and blue lines show the control and the sensitivity experiment, respectively.

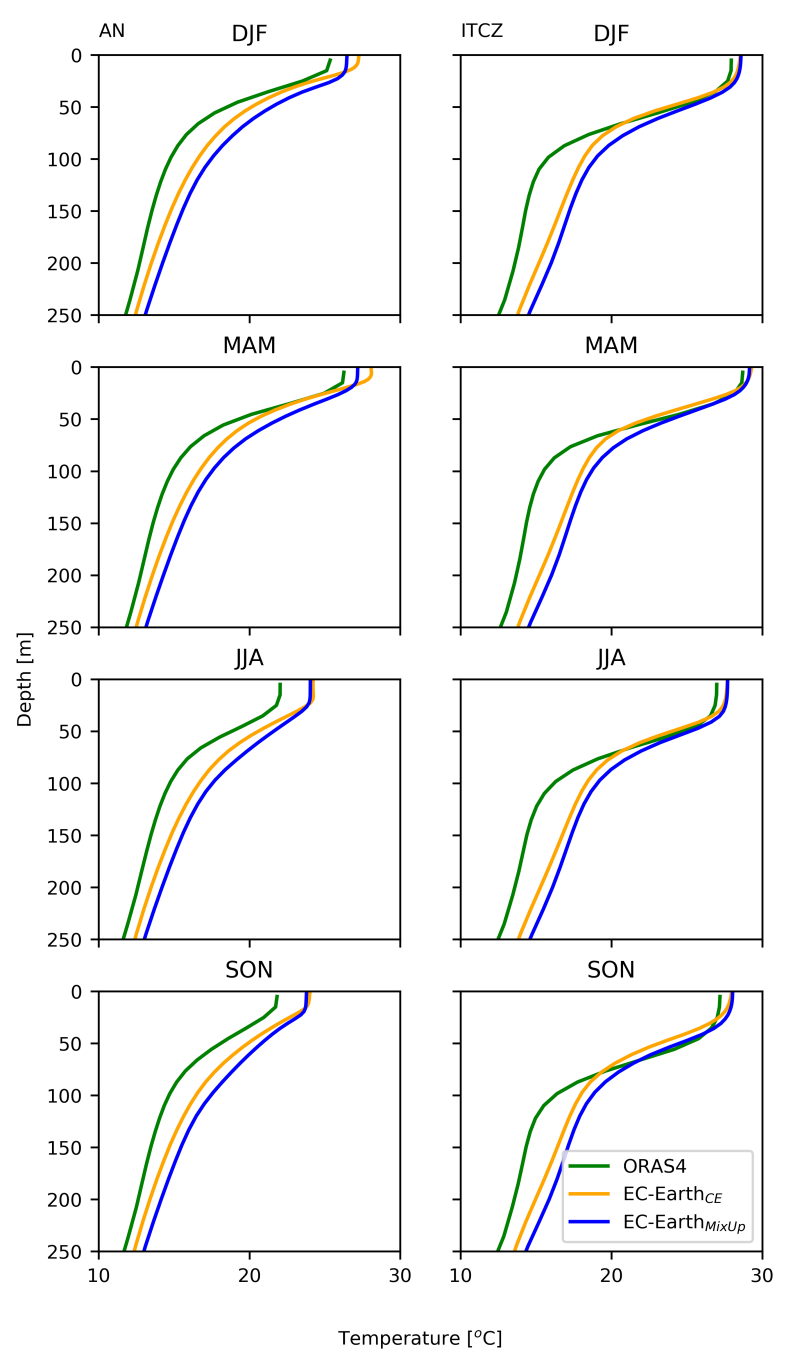

value for the vertical eddy diffusivity in the ocean model is $1.2 \cdot 10^{-5} \frac{\mathrm{m}^{2}}{\mathrm{~s}}$. The temperature gradient between the depth of the maximum warming at $50 \mathrm{~m}$ and the maximum depth at which we still observe warming $(1 \mathrm{~km})$, is approximately $20^{\circ} \mathrm{C}$. According to the law of diffusion, $\frac{\Delta T}{\Delta t}=-A_{v t} * \frac{\Delta T}{\Delta z^{2}}$, after 45 years of runtime an ocean column of $950 \mathrm{~m}$ below the maximum warming could have warmed by $\approx 0.4^{\circ} \mathrm{C}$. This is the order of magnitude of warming that we observe below the well mixed layer and thermocline.

In the ITCZ box surface temperatures in MixUppr cool less in comparison to Control ${ }_{p r}$ than in the AN box (Figs. 4.10 and 4.11). The upper 15-20m cool slightly throughout boreal spring and the beginning of summer (Fig. 4.11a). Below the cooled upper layer there is a warming of subsurface water. The maximum of this subsurface warming lies 
below the layer in which vertical mixing is enhanced (Fig. 4.11b). Because the mean enhanced mixing does not penetrate this layer, it seems that remote processes, rather than vertical mixing, play a dominant role. The warm signal closely follows the seasonal cycle of the depth of isopycnals in these layers. Ventilation along the isopycnal is known to reach the equatorial currents from the subtropical gyre via the Brazil Current (Hazeleger et al., 2003). While the AN box is partly untouched by advection, as it is located in the so-called shadow zone (Luyten et al., 1983; Pedlosky, 1983), waters from the subtropical Atlantic reach the ITCZ box through subsurface currents and may cause warming (Fig. 4.9). Shoaling plays a smaller role than in the AN box (average shoaling in ITCZ box at 1025 $\mathrm{kg} / \mathrm{m}^{3}: 6.7 \mathrm{~m}$, in the AN box: $\left.8.7 \mathrm{~m}\right)$.

Figure 4.11: Differences in the upper ocean column between the sensitivity and the control experiments in the ITCZ box. The left panel depicts the box average change in temperature for each month of the year, the center panel the amplification of the vertical eddy diffusivity parameter $A_{v t}$. The right hand panel shows temperature difference throughout the year with depth in colours, and the isopycnals in contours $\left[1000-\mathrm{x} \mathrm{kg} / \mathrm{m}^{3}\right]$. The solid lines are the isopycnals in the control experiment, the dashed lines the isopycnals in the sensitivity experiment.
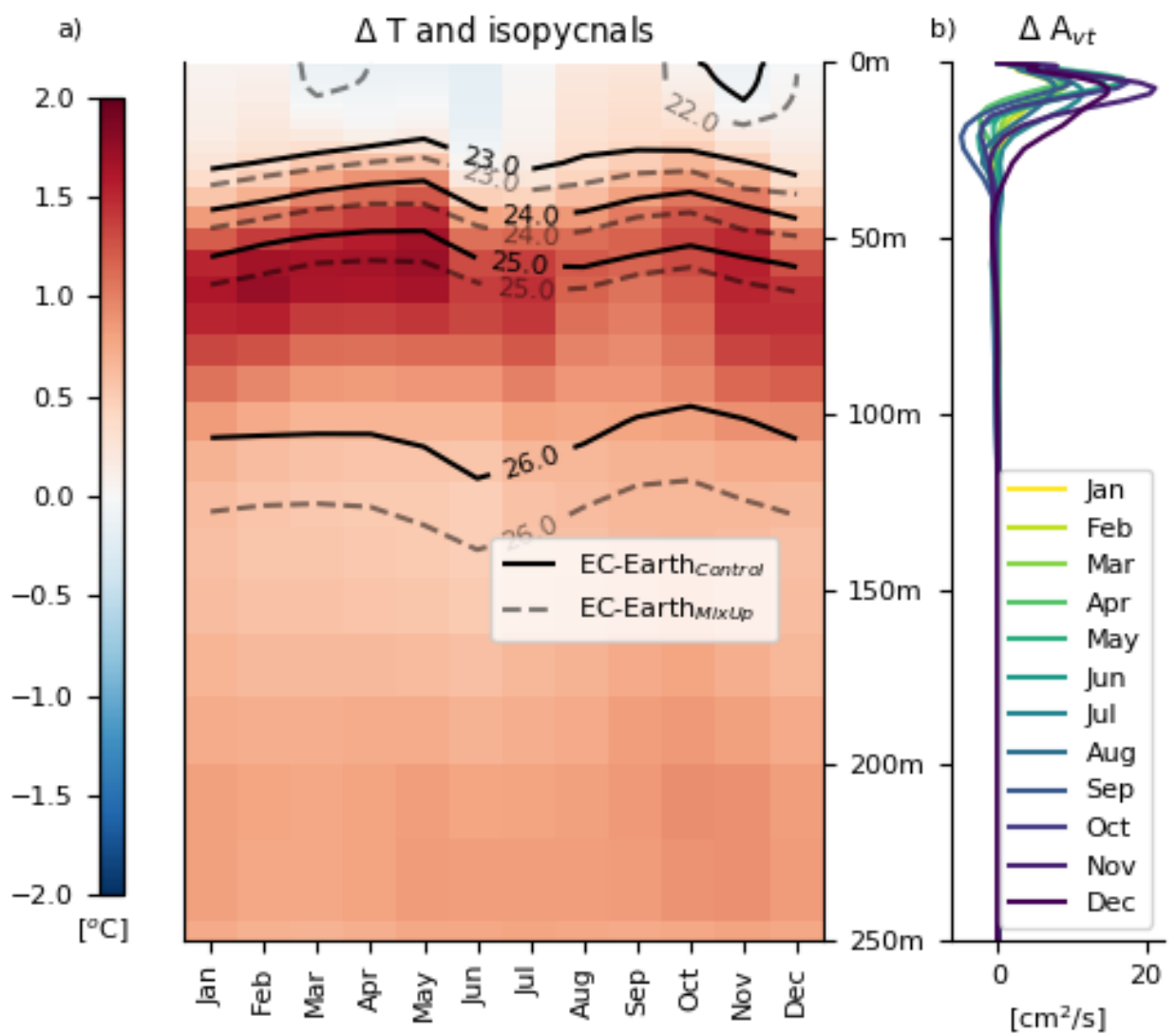
Impact of enhanced vertical mixing on the atmosphere in present day climate

Coupled to SST changes, the atmospheric circulation responds to the parameterization change. In boreal winter and spring, cooler SST in the southern part of the tropical Atlantic lead to a larger meridional SST gradient in MixU $\mathrm{p}_{p r}$. In line with the increased gradient, cross equatorial winds strengthen in those seasons, which reduces the wind bias seen in Control ${ }_{p r}$ (Figs. 4.12 and 4.4). In winter, this is most prominent between the equator and up to $30^{\circ} \mathrm{N}$ and east of $30^{\circ} \mathrm{W}$. In spring, the bias is reduced over most of the basin. The improved meridional temperature gradient and cross equatorial winds reduces the precipitation bias. In winter, the north-eastern part of the ITCZ strengthens and the spurious southern part of the ITCZ lessens. In spring, when the change in SST and wind is largest, the ITCZ moves northward. The negative precipitation bias of up to $4 \mathrm{~mm} /$ day in Control $_{p r}$ is halved in MixUppr , and greatly reduced in spatial extent. Similarly, the strength and extent of the southern part of the model ITCZ reduces beneficially by up to $3 \mathrm{~mm} /$ day.

Figure 4.12: Seasonally stratified sea surface temperature differences between ECEarth $_{\text {Control }}$ and EC-Earth $\mathrm{MixUp}_{\mathrm{p}}$ in present day climate in colours, differences of the wind vectors (arrows) and precipitation in contours. The control experiment is subtracted from the sensitivity experiment, such that negative (positive) values show decreased (increased) temperature and precipitation in MixUp compared to the control.
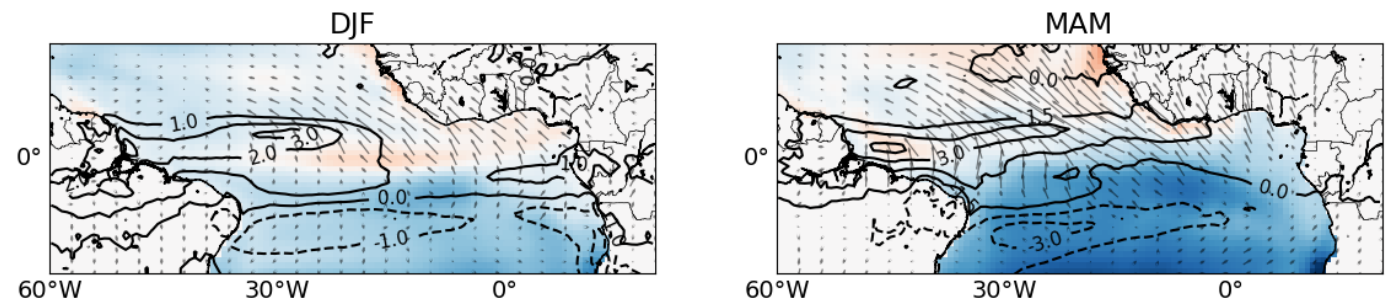

$2 \mathrm{~m} / \mathrm{s}$
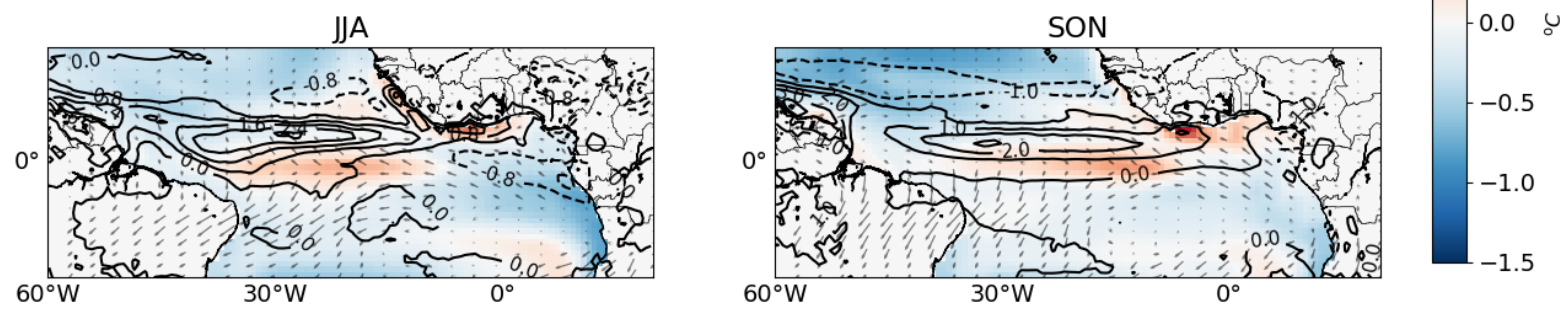

Throughout boreal summer and fall, SST increase along the equator in MixUp $p_{p r}$ compared to Control $_{p r}$ (Fig. 4.12). Locally, the westerly surface wind bias reduces. To the south of the warmer sea surface, the northerly wind bias remains. Above, and slightly north of, the region of increased SST precipitation is increased in the sensitivity experiment. In summer and fall, the ITCZ moves northward in ERA-interim (Fig. 4.3). In both the control and the sensitivity simulations, the seasonal displacement of the warm band is smaller than observed. In summer, the strength of the ITCZ is overestimated in both 
simulations. In the increased ocean vertical mixing experiment, the ITCZ is even stronger than in Control $_{p r}$, which leads to a larger positive precipitation bias.

In boreal fall, surface temperatures north of the simulated ITCZ are cooled in MixUp $\mathrm{p}_{p r}$, which increases the meridional gradient between the ITCZ and to the north of it. Therefore, the rain band is trapped in its northward movement and remains too far south. The ITCZ becomes narrower between the meridional temperature gradients, leading to a larger negative bias in the north and a stronger positive bias at the center of the simulated ITCZ. In the southwest of the tropical Atlantic, the positive alongshore wind bias at the coast of Brazil increases, but the bias west off the African coast is reduced, even though SSTs change only slightly.

Summarising, in winter and spring the circulation and precipitation patterns, along with SST, are improved in MixUp $\mathrm{p}_{p r}$. In boreal summer and fall there are improvements in the circulation and SST, but not in the location and strength of the ITCZ. Especially in fall the ITCZ is captured better by the control experiment.

Our analysis shows how sensitive the coupled system is to the choice of ocean vertical mixing parameterization. There are substantial differences between $\mathrm{MixUp}_{p r}$ and $\mathrm{Control}_{p r}$ in present day climate. In the following section, we investigate the effect of enhanced vertical ocean mixing on projected future climate.

\section{Tropical Atlantic Climate Change}

The tropical Atlantic region is relevant for science and society, hence there is considerable interest in how tropical Atlantic climate will react to the global increase of greenhouse gas (GHG) concentrations. Projected climate change and model sensitivity to GHG forcing has been investigated globally and regionally. In the tropical Atlantic, climate models do not agree on the response to increasing GHG. Precipitation changes are particularly uncertain. While some models project drying above the tropical Atlantic and on the surrounding continents, especially of the Sahel region, others project wetting of the North African subcontinent (Held et al., 2005; Cook and Edward, 2006; Biasutti et al., 2008).

The mechanism by which the rainfall patterns in and around the tropical Atlantic change are the result of tropical Atlantic SST forcing as well as direct response to enhanced GHG concentrations (Biasutti et al., 2008; Mohino et al., 2011; Biasutti, 2013). Cook and Edward (2006) suggest that temperature change in the Gulf of Guinea is an important indicator for rainfall shifts connected to the West African Monsoon (WAM). RodríguezFonseca et al. (2011) stress the importance of the background state to reliably simulate the WAM variability.

In this section, we investigate the tropical Atlantic climate change response to RCP8.5 (Ri- 
ahi et al., 2011) in EC-Earth, and its dependence on the ocean vertical mixing efficiency. We first focus on the signal common to the control and the sensitivity experiment, before highlighting the differences.

As a response to increasing GHG forcing, tropical Atlantic SST increase by up to $4.5^{\circ} \mathrm{C}$ at the end of the century (Fig. 4.13). The warming is basin wide, but it is intensified along the equator and in the east, where the equatorial cold tongue is located. Tropical Atlantic surface winds weaken, and precipitation increases above the warmer sea surface. In winter, the ITCZ intensifies, especially in the west of the basin where SSTs increase. In spring and summer, the ITCZ shifts northward, in line with enhanced warming of the sea surface on the northern hemisphere and the associated enhanced interhemispheric temperature gradient.

The upper ocean column warms by up to $4.5^{\circ} \mathrm{C}$. In the AN box, the signal gradually decreases with depth below $50 \mathrm{~m}$ (Figs. 4.14a). In boreal spring and early summer, the subsurface warming peaks between $20-50 \mathrm{~m}$ below the surface. At this depth the temperature gradient is steepest in present day climate (Fig. 4.10), leading to a large warming signal when the mixed layer deepens. Surplus warming at the top of the column stabilises the ocean column. The turbulent eddy diffusivity coefficient is decreased in the future (Fig. 4.14b) compared to present climate (Fig. 4.8b), in line with enhanced upper ocean stratification.

In the ITCZ box, the subsurface warming consists of two parts: near the surface, in the upper $50 \mathrm{~m}$, and below the thermocline, between $70 \mathrm{~m}$ and $200 \mathrm{~m}$ (Fig. 4.15a). The warming in the upper $50 \mathrm{~m}$ can be explained directly by warming of the overlying atmosphere, due to enhanced GHG forcing. Warmer surface temperatures stabilise the upper ocean. The subsurface warming, on the other hand, cannot be explained by local influences. Vertical mixing is not active below $50 \mathrm{~m}$ (Fig. 4.15b), and hence cannot mix heat into the ocean interior. The subsurface warming is likely due to advection of remotely subducted warmer water.

To summarise, in both experiments, the sea surface and subsurface warm, winds weaken, and tropical precipitation increases. However, there are large differences between Control $_{f u}$ and MixUp $\mathrm{p}_{f u}$. In the following, we compare the differences between the two simulations at the end of the century.

Both the SST response and the increase of tropical Atlantic precipitation are strongly dependent on the vertical mixing parametrisation (Fig. 4.16). The projected SST signal is stronger in the $\mathrm{MixUp}_{f u}$ than in the control experiment (Fig. 4.13). Locally, the amplification of the warming signal reaches $1^{\circ} \mathrm{C}$, a considerable fraction of the warming $\left(4.5^{\circ} \mathrm{C}\right)$. Strong equatorial warming in $\operatorname{MixUp}_{f u}$ leads to an increased meridional SST gradient in winter. Associated to the warm sea surface, local rainfall is stronger in $\operatorname{MixUp}_{f u}$ than in $\mathrm{Control}_{f u}$ (Fig. 4.16). In spring, it is notably colder in the southern hemisphere in $\operatorname{MixUp}_{f u}$ than in Control $_{f u}$. This leads to a WES-type feedback (Chang et al., 1997), 
The effect of vertical ocean mixing efficiency on the tropical Atlantic in a 94 coupled global climate model.

Figure 4.13: Seasonally stratified sea surface temperature, wind and precipitation climate change signal at the end of the century (2070-2099) as compared to present day climate (19792009) under RCP8.5 forcing for EC-Earth Control $_{\text {and EC-Earth }}$ MixUp.
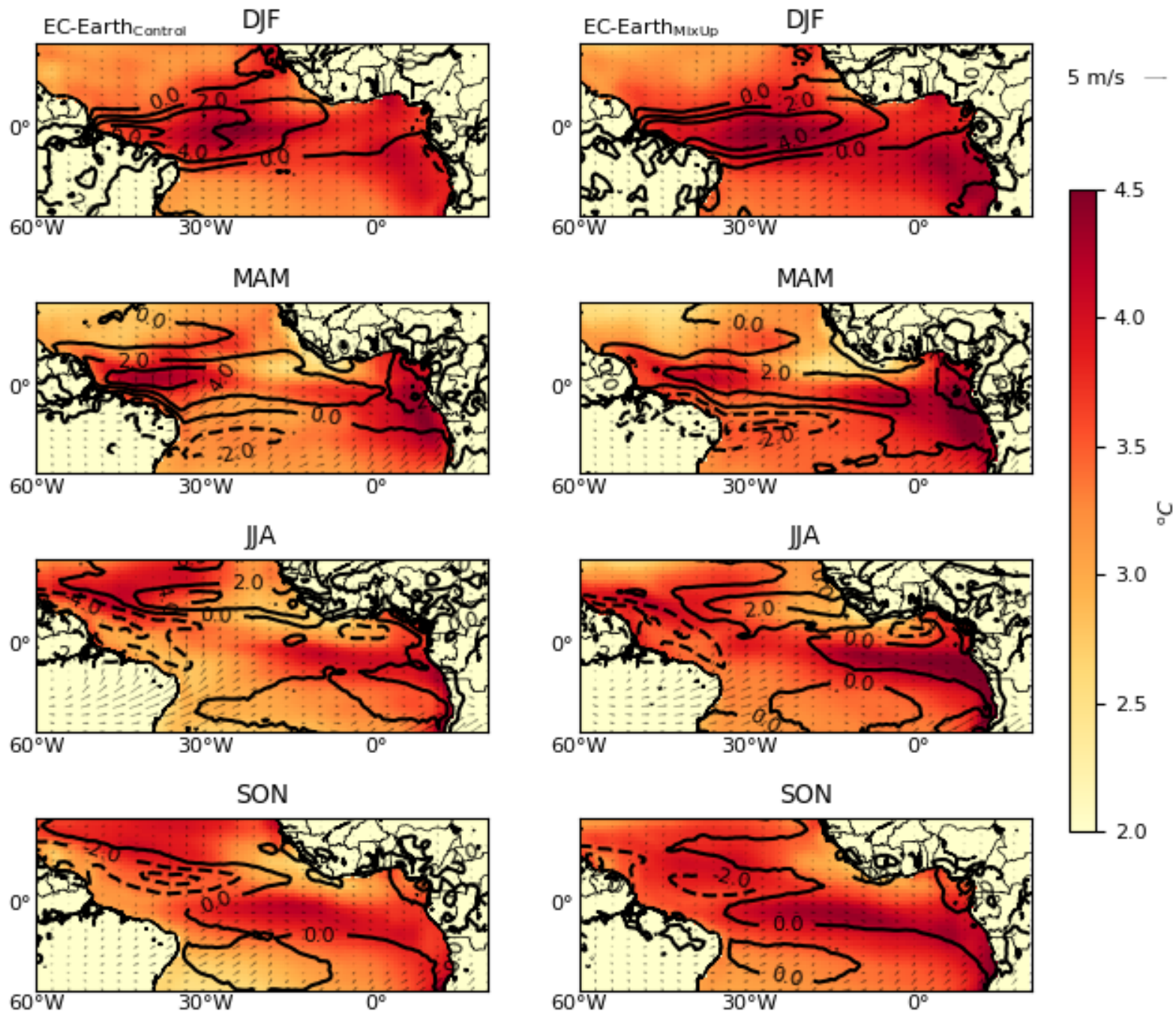

$60^{\circ} \mathrm{W}-30^{\circ} \mathrm{W}$

which enhances southerly cross-equatorial winds and shifts the ITCZ to the north. In summer, this signal is reversed. The southern hemisphere is warmer in MixUp $\mathrm{p}_{f u}$, and northerly winds are stronger, shifting the ITCZ to the south. This signal prevails in fall, albeit with a weaker amplitude. In both seasons, future precipitation over Africa is weaker in the enhanced ocean mixing experiment.

The differences between MixUp and Control are state dependent. That is, the effects are different in present day climate than in a future climate. While in summer and fall the southern hemisphere is warmer in $\operatorname{MixUp}_{f u}$ than in Control $_{f u}$ (Fig. 4.16), it is colder in MixUp $p_{p r}$ than in Control $_{p r}$ (Fig. 4.12). This highlights the system's sensitivity to vertical 
Figure 4.14: Climate change signal of the ocean column in the AN box in the control experiment (above), and the sensitivity experiment (below). Panels a and c show ocean temperature in colours, density differences in contours, and panels $\mathrm{b}$ and $\mathrm{d}$ depict differences in eddy diffusivity $\mathrm{A}_{v t}$.

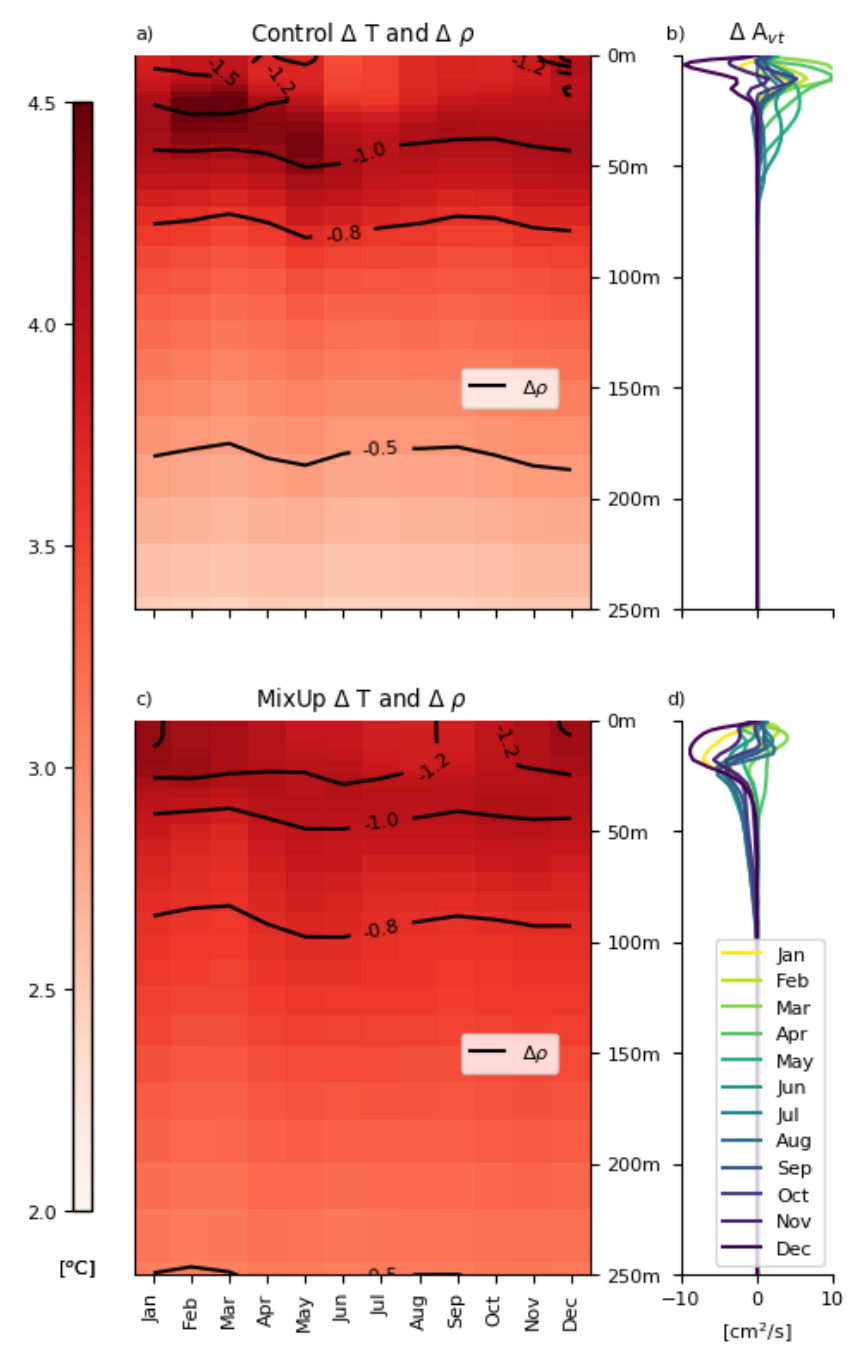

ocean mixing.

As described above, the southern hemisphere in $\operatorname{MixUp}_{f u}$ is relatively cold in boreal winter and spring, and warm in boreal summer and fall, when compared to Control $_{f u}$. This translates to a suppressed seasonal cycle along the equatorial band in the sensitivity experiment (Fig. 4.17a, b). The differences along the equator are enhanced in future climate as compared to present day climate (Fig. $4.17 \mathrm{c}, \mathrm{d}$ ). While the seasonal cycle weakens in the ITCZ box (close to the equator), it strengthens in the AN box (not shown).

Large differences between the two simulations also exist below the ocean surface. In the AN box, the subsurface warming in $\operatorname{MixUp}_{f u}$ (Fig. 4.14c) is stronger and extends to 
Figure 4.15: Climate change signal of the ocean column in the ITCZ box in the control experiment (above), and the sensitivity experiment (below). Panels a and c show ocean temperature in colours, density differences in contours, and panels $\mathrm{b}$ and $\mathrm{d}$ depict differences in eddy diffusivity $\mathrm{A}_{v t}$.

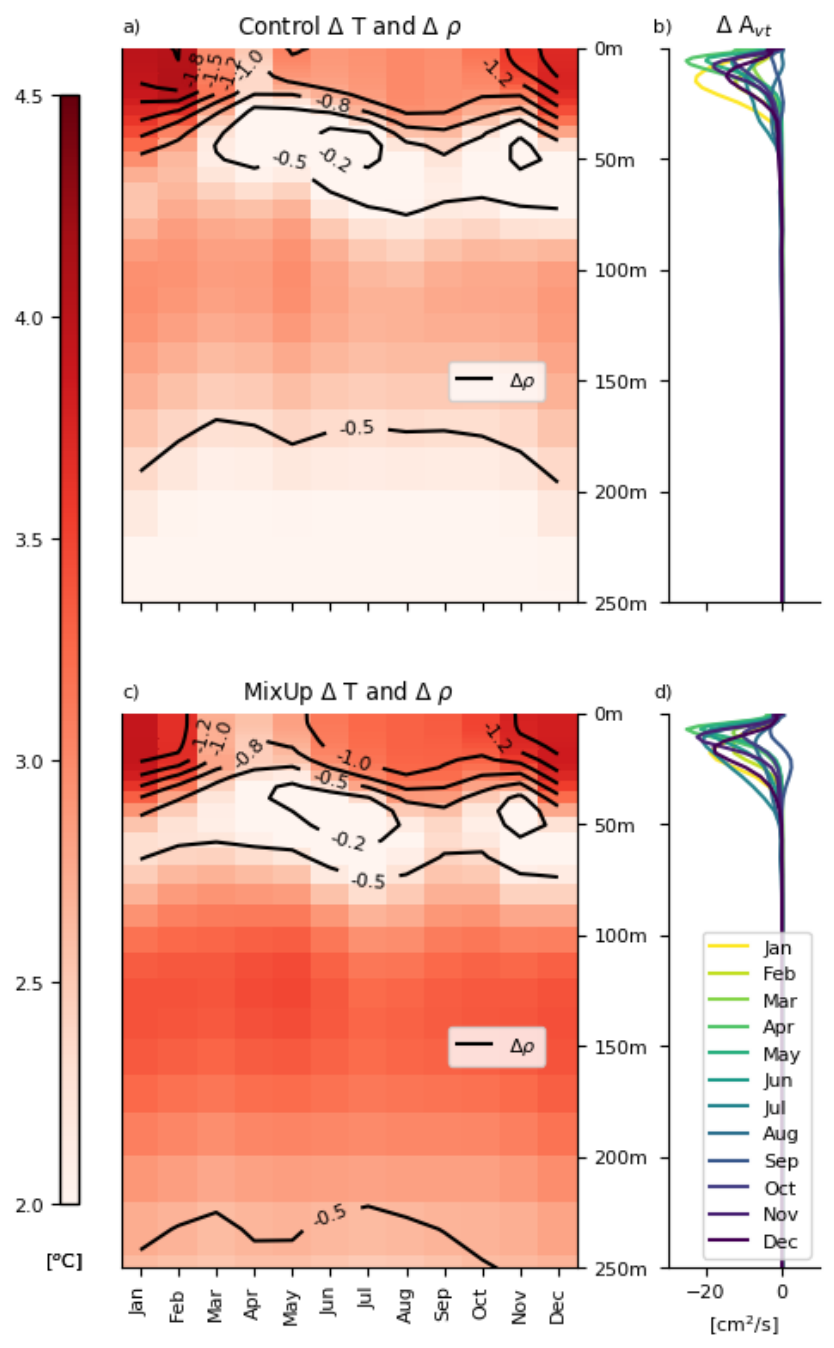

greater depths than in Control $_{f u}$ (Fig. 4.14a). Due to the stronger vertical mixing, more heat is transported downwards from the surface. As a result, stability in the upper ocean increases less. Note that while the vertical eddy diffusivity in $\operatorname{MixUp}_{f u}$ decreases at the end of the century (Fig. 4.14d), the decrease is a factor two smaller than the differences between the two experiments in present day climate (Fig. 4.14b). Mixing is still stronger in $\mathrm{MixUp}_{f u}$ than in $\mathrm{Control}_{f u}$ at the end of the century.

Ocean temperatures in the ITCZ box increase more in $\mathrm{MixUp}_{f u}$ than in Control $_{f u}$, especially in boreal summer and fall (Fig. 4.15). Below the thermocline, the warming (likely due to advection) is amplified throughout the year. 
Figure 4.16: Seasonally stratified differences in sea surface temperature, wind vectors, and precipitation between EC-Earth $_{\mathrm{MixUp}}$ and EC-Earth $\mathrm{CE}_{\mathrm{E}}$ as before in Fig. 4.12, but for future climate (2070-2099).
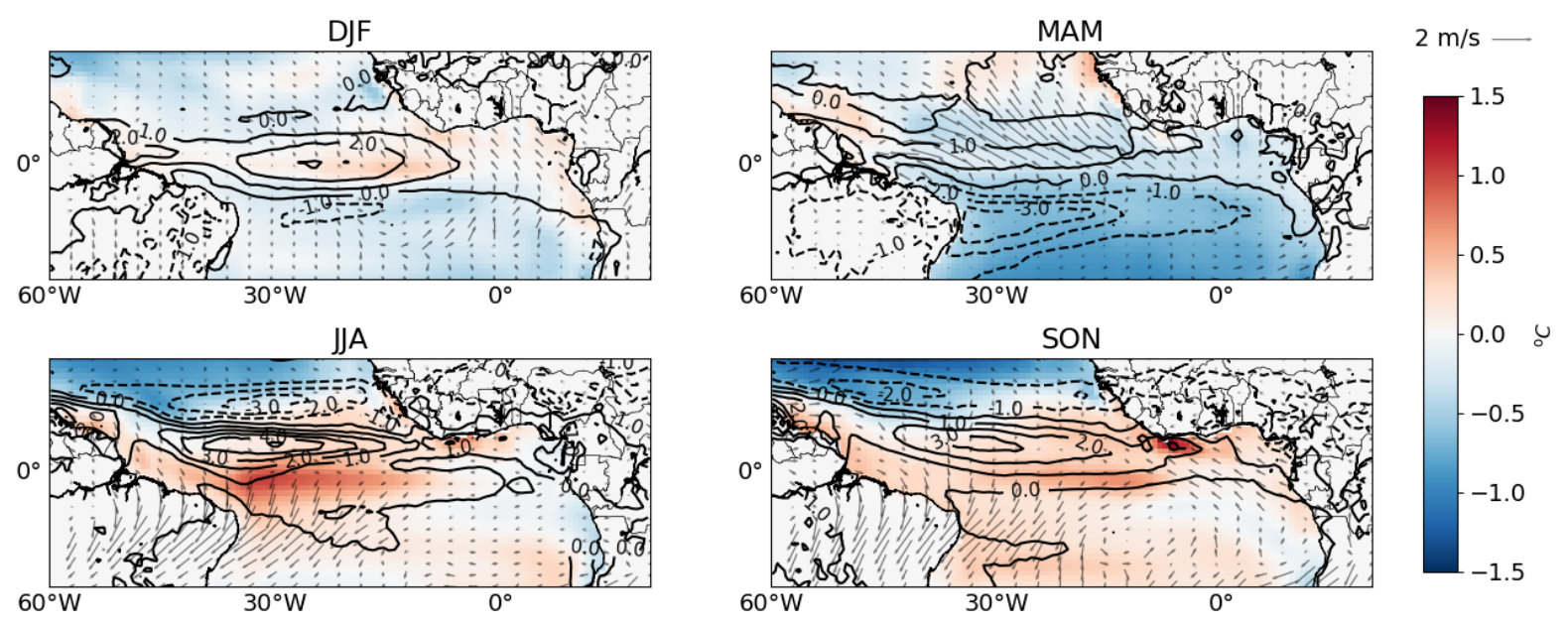

\subsection{Summary and Discussion}

EC-Earth, like most other state-of-the-art coupled global climate models, suffers from fast developing and persistent warm sea surface temperature biases in the eastern and east-equatorial tropical Atlantic (Richter and Xie, 2008; Wang et al., 2014; Richter et al., 2018, and others). These biases are present in the equilibrated state in climate simulations (Fig. 4.1), as well as in simulations initialised from estimates of the observed states after only 2-3 months of runtime (Fig. 4.2).

We analyse the upper ocean mixed layer heat budget from EC-Earth and reanalysis data for two regions displaying large SST biases and shallow mixed layer depths, and compared the individual contributions to the sea surface temperature evolution (Fig. 4.5). We show that unresolved subgrid-scale processes play a large role in the fast development of the tropical Atlantic warm bias in EC-Earth. Ocean vertical mixing is an important component of these small-scale processes which exerts sizable influence on SST, especially in regions where the mixed layers is shallow (Foltz et al., 2003; Planton et al., 2018, and others). We increase the the ocean vertical mixing efficiency in a historical climate simulation and in a climate projection with RCP 8.5 to test the system's sensitivity to the representation of this turbulent process.

The tropical Atlantic shows great sensitivity to ocean vertical mixing parameterisation, as summarised in Fig. 4.18. The present day climate SST bias in the tropical Atlantic is reduced under enhanced mixing (Fig. 4.6). Following the SST improvement, atmospheric biases in precipitation and winds also decrease (Fig. 4.12). EC-Earth suffers from 
Figure 4.17: Sea surface temperature (colours) and precipitation (contours every $2 \mathrm{~mm}$ ) in future climate $(2070-2099)$ along the equator $\left(3^{\circ} \mathrm{N}-3^{\circ} \mathrm{S}\right)$ for EC-Earth $\mathrm{Control}_{\text {and }} \mathrm{EC}$ Earth $_{M i x U p}$, and the differences between the two experiments (contours indicate $1 \mathrm{~mm}$ precipitation differences).
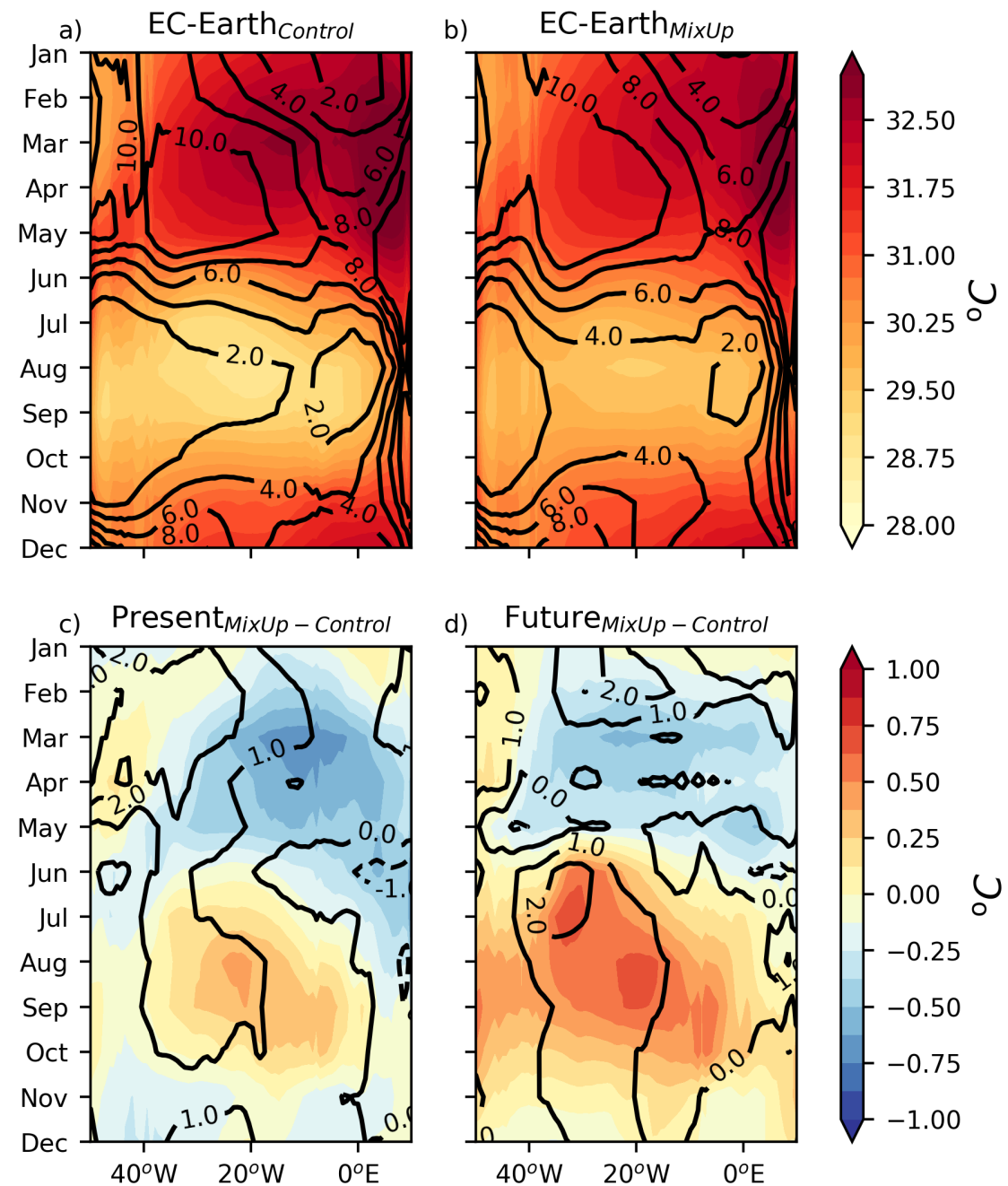

relatively small atmospheric biases in the tropical Atlantic compared to other CGCMs (Fig. 4.3). However, it simulates excess precipitation to the south of the ITCZ, similarly to other models (Biasutti et al., 2006; Breugem et al., 2006; Lin, 2007, and others), and it suffers from a weak (zonal) wind bias. Especially the bias in the western equatorial region is small, which is deemed important for inter-annual variability in connection with the Bjerknes Feedback (Zebiak, 1993; Ruiz-Barradas et al., 2000; Keenlyside and Latif, 2007; Deppenmeier et al., 2016). Cross equatorial winds as well as precipitation are beneficially increased under enhanced mixing (Fig. 4.12).

Subsurface ocean temperatures are also affected by the enhanced mixing. Off the coast of Angola and Namibia, in the AN box, the difference between MixUp $\mathrm{p}_{p r}$ and $\mathrm{Control}_{p r}$ 
Figure 4.18: Schematic of changes in the climate system under enhanced vertical mixing. Sea surface temperatures cool, the penetration depth of the cooling is region-dependent. Mixing increases more strongly in the south eastern tropical Atlantic than along the equator in the central Atlantic. Meridional surface wind (orange) convergence increases and the ITCZ becomes stronger and narrower. Easterly surface winds (grey) south of the equator increase.
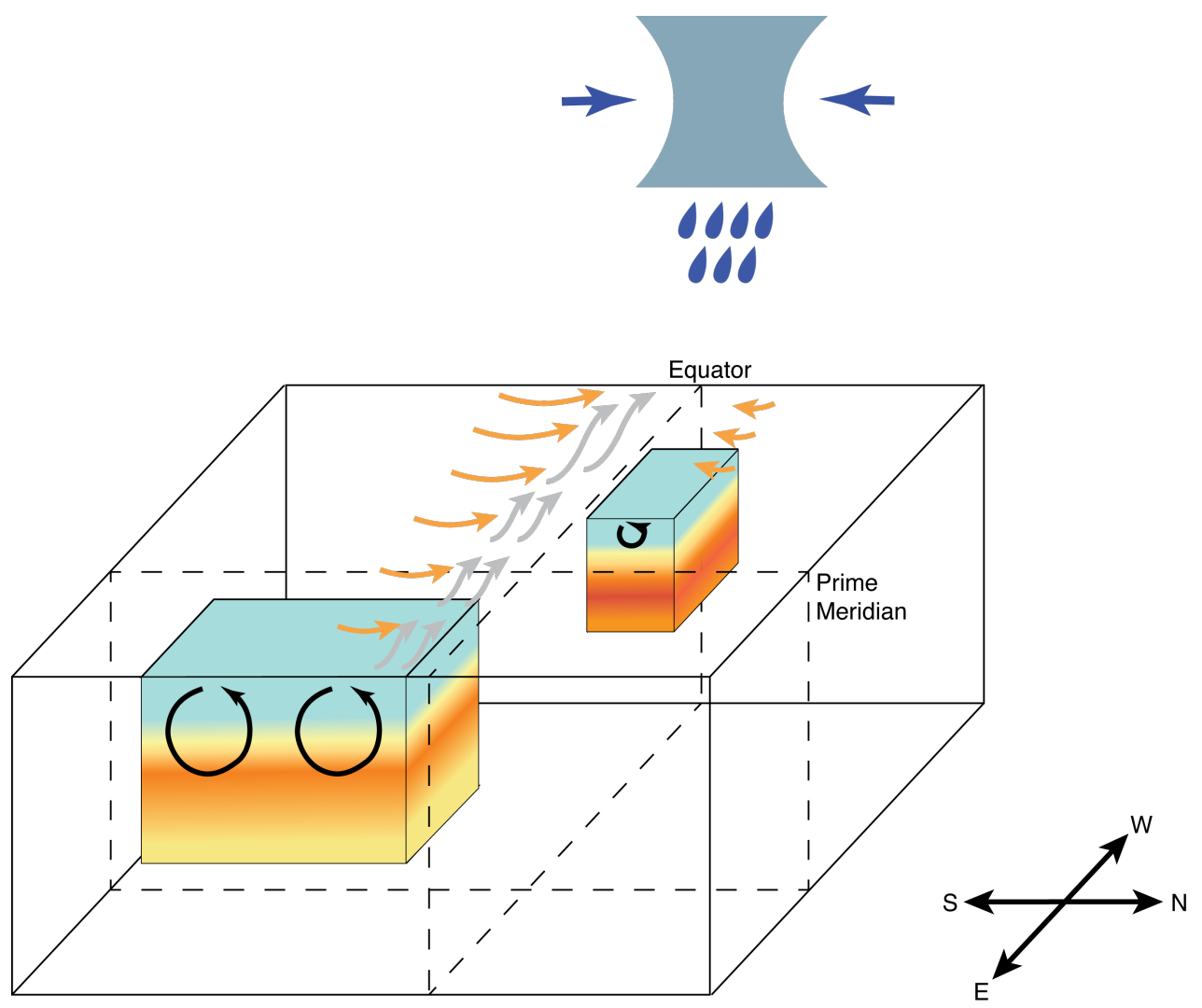

manifests as a vertical dipole in the upper 50-75 $\mathrm{m}$ of the ocean column. The depth coincides with the layer in which vertical eddy diffusivity is active. The dipole likely develops due to heat redistribution in the mixed layer. Enhanced mixing transports heat to the bottom of the thermocline, from where it can only reach the deeper ocean via slow diffusion processes. Apart from this local effect, advection along isopycnals might transport warmer water to layers at or below thermocline depth (Fig. 4.9a), and deepening of the isopycnals by shoaling or heaving can lead to temperature changes (Fig. 4.9b). At the depth of the thermocline, where the temperature gradient is large, slight heaving or shoaling of the isopycnal can lead to large temperature changes. A combination of local and remote effects likely play a role in warming the water at the bottom of the mixed layer in this region.

In the ITCZ box, the surface cooling in MixUppr is weaker than in the AN box with respect to Control $_{p r}$. Vertical eddy diffusivity increases in the upper $40 \mathrm{~m}$ of the ocean column. Below the slightly cooled surface layer, and below the layer in which $A_{v t}$ increases, 
$\mathrm{MixUp}_{p r}$ is warmer than Control $p$. The warm signal follows the $1025 \mathrm{~kg} / \mathrm{m}^{3}$ isopycnal closely, which suggests advective processes along this isopycnal (Fig. 4.9a). In both boxes, the ocean is warmed down to $1 \mathrm{~km}$. This deep warming is attributed to a slow vertical diffusion processes.

We further investigate projected climate change under the RCP8.5 forcing scenario in EC-Earth by performing two climate projection experiments, Control $f u$ and MixUp $\mathrm{p}_{f u}$. In both experiments, the tropical Atlantic warms, winds weaken and maritime precipitation increases. While there are similarities between the two projections, the climate change signal is sensitive to the vertical mixing parameterization. SST and precipitation increase more in the $\operatorname{MixUp}_{f u}$ than in Control $_{f u}$. The climate change signal is also dependent on the season. MixUp $\mathrm{p}_{f u}$ remains cooler than Control $_{f u}$ in winter and spring. In boreal summer and fall, $\mathrm{MixUp}_{f u}$ is warmer than Control $_{f u}$, especially in the southern hemisphere.

The subsurface climate change also dependends on the vertical mixing parametrisation, especially in the AN box. In $\mathrm{MixUp}_{f u}$ the entire upper ocean column is warmed almost uniformly, while in Control $_{f u}$ the largest warming can be seen at the depth of the thermocline.

In this study we highlight the large impact of ocean vertical mixing on the coupled system. In both present day, as well as future climate, enhanced vertical mixing strongly influences the ocean (sub)surface, and the atmospheric circulation. Changes at the sea surface are especially large in regions where the mixed layer is shallow. Enhancing vertical mixing reduces the present day tropical Atlantic SST warm bias, as well as biases in precipitation and in the atmospheric circulation. Further research and collaboration between modelers and observationalists are necessary to better constrain the important control that vertical ocean mixing asserts on the climate system. 
Chapter 5

Coupled ocean mixing and cloud feedbacks in the tropical Atlantic 


\section{Abstract}

The pronounced seasonal sea surface temperature (SST) variability in the Eastern tropical Atlantic exerts strong influences on other components of the climate system, such as the Intertropical Convergence Zone and the West African monsoon. However, key physical mechanisms of SST variability are not fully known. We combine in-situ observations, satellite observations, and reanalysis data to demonstrate that feedbacks involving cloud characteristics and upper ocean vertical mixing affect climate variability. These relatively fast processes act on daily to weekly time scales. SST anomalies influence cloud cover and, thereby, surface shortwave radiation. The latter affects upper ocean thermal stability and the strength of vertical ocean mixing, which explains $35 \%$ of the regional SST variance. This ocean-cloud feedback is positive off the coast of Angola and Namibia, whereas it is negative in the Intertropical Convergence Zone. Correct model-representation of this feedback and its geographical imprint is crucial for improving forecasts and climate projections. 


\subsection{Introduction}

Climate variability and weather in the tropics are determined by strong coupling between ocean and atmosphere (Chang et al., 1997; Zebiak, 1993; Xie and Carton, 2004; Chang et al., 2006). The upper ocean turbulently mixed layer is shallow in the tropics such that incoming radiation and turbulent surface heat fluxes can strongly influence the upper ocean temperature. Additionally, the impact of wind driven mixing, which brings cold water into the shallow ocean mixed layer, and wind-driven upwelling of cold water can be large. These processes play a key role in coupled feedbacks. This forms the basis of forecast skill in initialized numerical model forecasts up to seasonal time scales. The importance of upper ocean processes, including turbulent mixing, is evident from previous studies (Foltz et al., 2013, 2018). Here we expand on these studies and assess coupled ocean-atmosphere feedbacks at relatively short daily to monthly time scales.

Coupled feedbacks can occur because the tropical atmosphere is receptive to changes in ocean surface temperature. On the one hand, deep convection and associated cloud cover occur above SSTs of 26-28 C (Gadgil et al., 1984; Fu et al., 1994; Sud et al., 1999; Johnson and Xie, 2010; Small et al., 2011). Exceeding this threshold triggers convective cloud formation. On the other hand, over colder water, such as ocean upwelling regions where air subsides, the atmosphere is stable and allows shallow stratocumulus clouds to form. Over these regions cloud formation is sensitive to changes in the atmospheric stability that is affected by SST (Klein and Hartmann, 1993; Yu and Mechoso, 1999; Clement et al., 2009; Eastman et al., 2011; Bellomo et al., 2015). Resulting changes in cloud cover fraction influence the surface radiation budget.

Though advances in understanding tropical Atlantic variability (TAV) have been made (Richter et al., 2013; Brandt et al., 2014; Richter et al., 2014a; Lübbecke et al., 2010, 2014; Polo et al., 2013), many processes are not well understood and state-of-theart numerical weather prediction and climate models are unable to reliably simulate and predict tropical Atlantic SST variability (Richter and Xie, 2008; Davey et al., 2002; Chang et al., 2007; Toniazzo and Woolnough, 2014; Xu et al., 2014b). Because of the large impact of SST on tropical atmospheric circulation, this deteriorates seasonal forecasts and climate projections for the tropical Atlantic and the West African countries (RodríguezFonseca et al., 2011). Also, the region is of interest for climate projections, because of the important role of the ITCZ in the general circulation and because variations in simulated marine stratocumulus clouds contribute to the spread in climate sensitivity (Bony and Dufresne, 2005).

Here, we investigate and quantify coupled feedbacks with statistical analyses using in situ, reanalysis and satellite data in two climatic regimes in the tropical Atlantic where active coupling may take place, namely the ITCZ region and the eastern upwelling region. 


\subsection{Data and methods}

We consider two regions in the tropical Atlantic where air sea coupling can be strong. The conditions where this can occur are: a shallow mixed layer, cloud cover and SST cooling in summer due to upwelling. These conditions are met in two key regions (Figure 5.1).

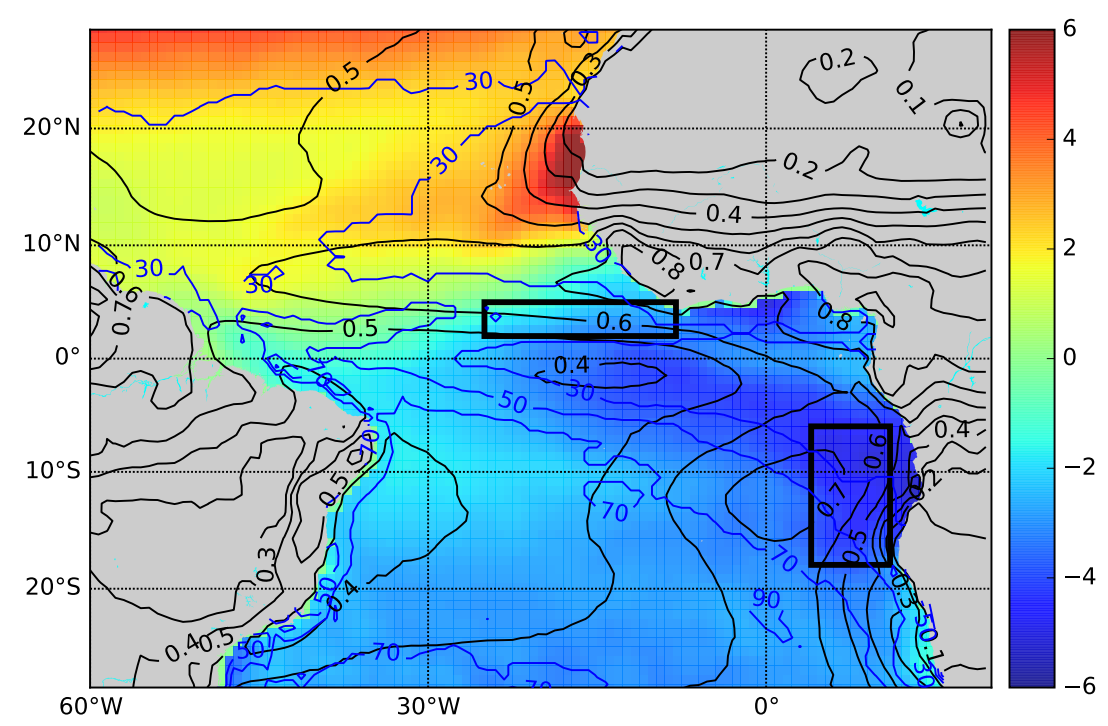

Figure 5.1: Average tropical Atlantic sea surface temperature cooling $\left[{ }^{0} \mathrm{C}\right]$ from May to August in colours, total cloud cover [fraction] in black contours, mixed layer depth [m] in blue contours. The latter two quantities are May-August averages. All quantities are derived from reanalysis data in 2000-2009. Black boxes indicate the two regions investigated in this paper (from left to right ITCZ and AN).

The first region is located off the coast of Anogla and Namibia (AN box, $18-6^{\circ} \mathrm{S}, 4-11^{\circ} \mathrm{E}$ ). Here, the dominant cloud cover type is shallow stratocumulus. The second region is located underneath the southern extension of the ITCZ (ITCZ box, 25- $8^{\circ} \mathrm{W}, 2-5^{\circ} \mathrm{N}$ ). Here, deep convective clouds are dominant. Throughout May to August the equatorial cold tongue develops and peaks, cooling SST in both boxes by up to $5{ }^{\circ} \mathrm{C}$. In these regions (inter-)annual SST variability is large.

For the in situ data analysis, the temporal evolution of the ocean column temperature at $8^{\circ} \mathrm{S}, 6^{\circ} \mathrm{E}$ is obtained from ten minute resolution PIRATA data (Servain et al., 1998). Mixed layer depth variations are derived using a temperature threshold $T_{h} \leq S S T-0.1$. Upper ocean stratification is estimatead from the difference of ocean temperature at surface level and $20 \mathrm{~m}$ depth.

We also consider the upper ocean heat budget for a well mixed layer with thickness $h$ :

$$
\partial_{t} T_{s}=\frac{Q}{h \rho_{w} c_{p}}-w \partial_{z} T-u \partial_{x} T-v \partial_{y} T-\partial_{t} T_{s m i x}+R
$$


In equation 5.1, $\partial_{t} T_{s}$ is the temperature evolution in the upper mixed layer approximated by SST evolution, $Q$ is the net surface flux into the mixed layer, $u, v$, and $w$ are the horizontal and vertical current velocities. $\rho_{w}$ is the density of seawater, $c_{p}$ its heat capacity, and $h$ is the mixed layer depth. $\partial_{t} T_{s m i x}$ is the upper ocean vertical mixing, estimated as:

$$
\partial_{t} T_{s m i x}=\frac{\alpha}{h^{2}} u_{*}^{3},
$$

following a bulk mixed layer approach (Sterl and Hazeleger, 2003; Niiler, 1977). In equation (5.2) $\alpha$ is a mixing efficiency parameter with value $10^{3}, h$ is the mixed layer depth. The friction velocity $u_{*}$ is defined by $u_{*}=\sqrt{\frac{|\tau|}{\rho_{a}}}$, with the magnitude of the wind stress vector $|\tau|$ and the density of air $\rho_{a}$. Note that the effect of ocean stratification on the mixing efficiency is not visible in this heat budget because of compensating terms. The residual terms $R$, such as diffusion, are not resolved in this calculation.

Upper ocean vertical mixing is estimated from three hourly wind stress data from ERAInterim (Dee et al., 2011). TropFlux (Kumar et al., 2012) daily surface flux data is used for the net heat flux component. The ocean mean advection components are calculated from monthly ORAS4 ocean reanalysis data (Balmaseda et al., 2013) due to the unavailability of long time series ocean data with high temporal resolution. The mixed layer depth $h$ is estimated as for PIRATA data. Mixed layer depths obtained from a density criterion agree well with the depth obtained from the temperature criterion (not shown). Error bars in the heat budget are estimated from one standard deviation around the interannual mean. Errors in the residual term are assumed to be dominated by the largest error of the other terms.

Feedbacks are studied used lagged correlation analysis. Following work from Frankignoul and Hasselmann (1977) and applications thereof, e.g. von Storch (2000); Keenlyside and Latif (2007); Breugem et al. (2007), lagged correlations between oceanic and atmospheric variables, surface and radiative fluxes can indicate coupled feedbacks. Data are smoothed with a five day running mean and then integrated over $1-8$ days before performing the correlation analysis. Different than earlier studies we use a range of time scales to study shifts in coupling over time.

\subsection{In situ data analysis}

We first study in situ data to obtain insight in the role of the atmospheric forcing and the oceanic vertical structure in mixing and the effects on SST. High temporal resolution data from the PIRATA buoy array in the tropical Atlantic (Servain et al., 1998) shows strong upper ocean temperature and mixed layer depth fluctuations, even over the course of a day (Figure 5.2). This indicates strong upper ocean mixing events which can impact SST, as noted by Foltz et al. (2018) as well for some PIRATA buoy locations. 

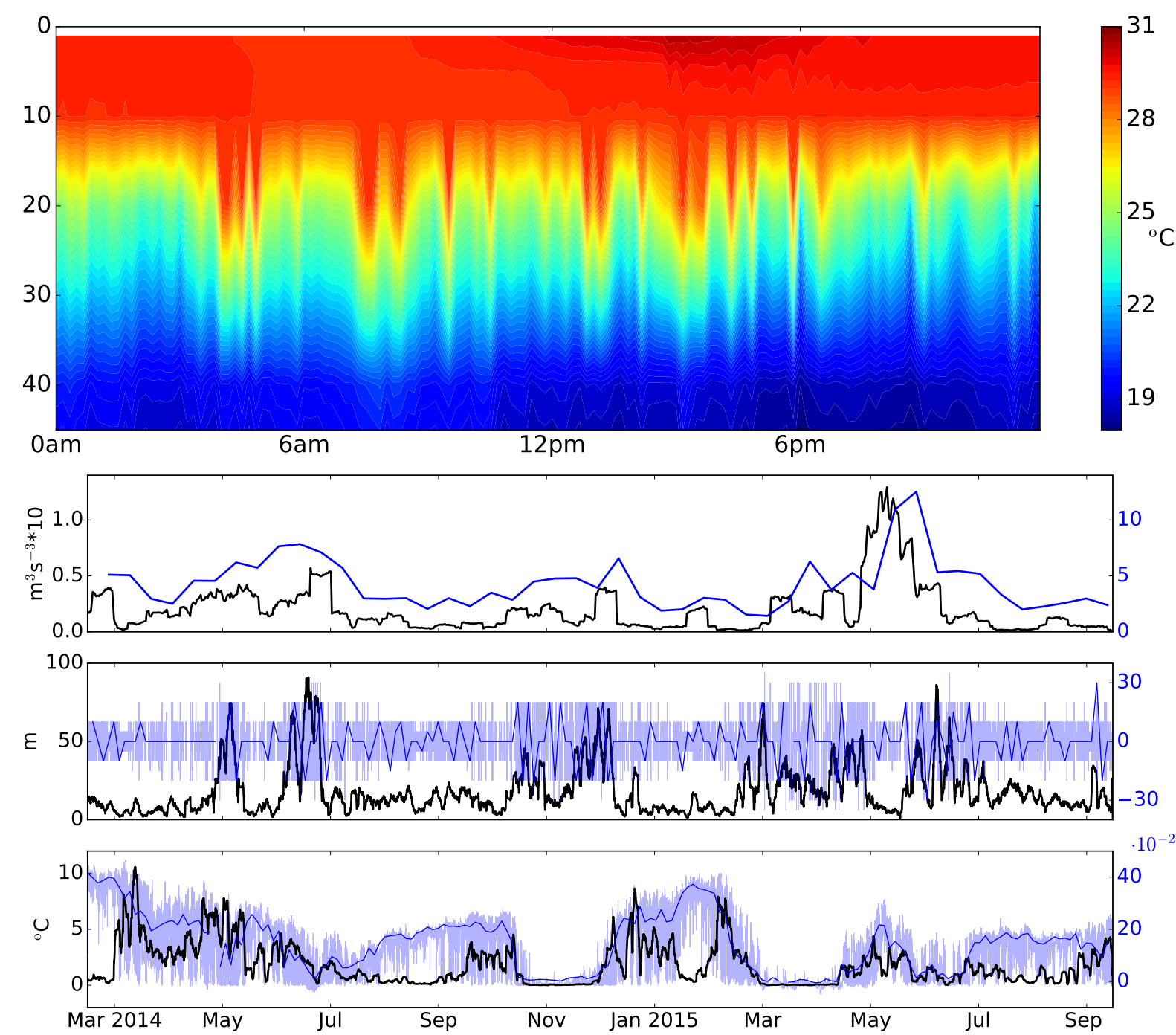

Figure 5.2: Top panel: high temporal resolution (10 minutes) temperature $\left[{ }^{0} \mathrm{C}\right]$ on the first of May 2014. Second panel: friction velocity cubed $\left[\frac{m^{3}}{s^{3}}\right]$ derived from ERA-Interim in the boreal summer of 2014, three day rolling variances in black (left ordinate), three day integrated differences in blue (right ordinate). Third panel: temporal mixed layer depth change $[\Delta \mathrm{m}]$ from one time step to the next derived from PIRATA data at $8^{\circ} \mathrm{S}, 6^{\circ} \mathrm{E}$. Blue and black lines as in second panel, light blue lines depict change per time step. Bottom panel shows upper ocean stratification $\left[\Delta^{0} \mathrm{C}\right]$ at the same PIRATA buoy in the same color coding as above. 

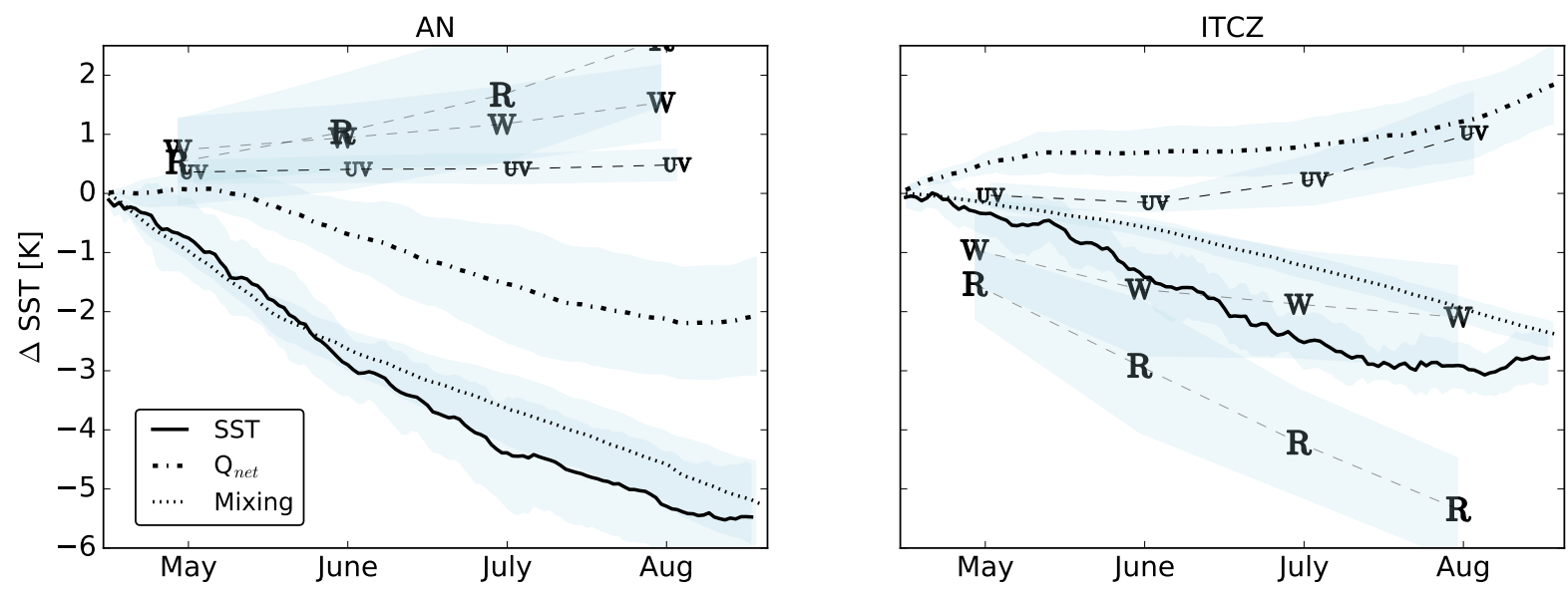

Figure 5.3: Mixed layer heat budgets of both boxes in Fig. 5.1. All contributions are accumulated over time and given in units of $\left[{ }^{0} \mathrm{C}\right]$. Sea surface temperature (solid), contribution of mechanical mixing (dotted), net surface heat fluxes (dashed-dotted), horizontal advection (UV marker), vertical advection (W marker), and the residual term (R) are shown, according to equation 5.1. Envelops show one standard deviation derived from interannual variability. For $\mathrm{W}, \mathrm{UV}$ and $\mathrm{R}$ the terms are estimated per month.

An estimate of turbulent energy input by wind and waves derived from ERA-Interim (Dee et al., 2011) indicates that mixing events cannot be attributed to strong wind energy input alone (Fig. 5.2). Large variance of the mixed layer depth, a proxy for mixing in the upper ocean (center panel Fig. 5.2) does not always coincide with large variance of friction velocity. For instance, strong mixing is not accompanied by large variance of friction velocity in November 2014. In this month, however, there is hardly any upper ocean stratification which is a favourable condition for effective wind driven mixing. On the other hand, despite strong wind input in June 2014, mixed layer variability still remains low. The stable stratification in this month could be both reason for, as well as a result of the suppressed mixing.

These results indicate a role for mixing and ocean stratification in short term SST variability, but causalities cannot be deduced from this data because the time series are too short. We will further quantify the heat budget and coupled feedbacks with reanalysis data.

\subsection{Reanalysis data analysis}

To shed light on the role of ocean processes in SST evolution, we show the upper ocean heat budget in both boxes in the cooling season when vertical ocean mixing and upwelling is large (see Figure 5.3). 

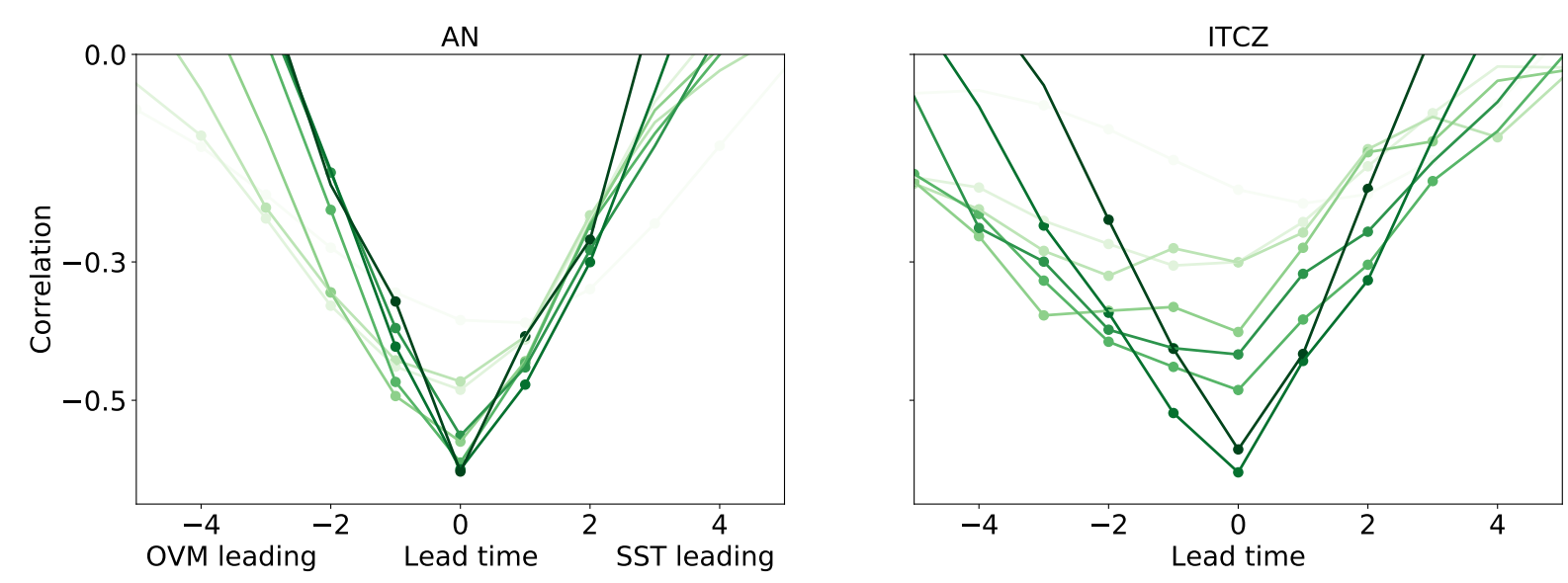

Figure 5.4: Lead lag cross-correlation between ocean vertical mixing (OVM) and sea surface temperature evolution. The shading from light to dark indicates the time scale over which the data is integrated, from one day in lightest shade to eight days darkest shade. Significant correlations at $95 \%$ confidence level are marked with a circle. The left panel shows the AN region, the right the ITCZ region.

Off the Angolan/Namibian coast the combined effect of outgoing longwave radiation and latent heat flux cools the mixed layer, in addition to cooling by mixing. Underneath the ITCZ incoming shortwave radiation dominates the net surface radiation budget and leads to warming of the mixed layer. Mean vertical upwelling as well as mean horizontal advection hardly influence the heat budget. Upwelling in the AN box unexpectedly slightly warms the mixed layer. However, we only consider monthly means and do not resolve mesoscale eddies. Convergence of heat by lateral eddy mixing can counteract the cooling by upwelling (Hazeleger et al., 2001). In the ITCZ box upwelling slightly cools the mixed layer. In both regions, the vertical mixing and the surface flux terms dominate. These results confirm earlier findings in Foltz et al. (2013, 2018) and Peter et al. (2006). Here, we extend the analysis to study coupled feedbacks.

Lead-lag correlations between SST evolution and ocean vertical mixing reveals a feedback between the two (Figure 5.4). Both terms are individually integrated over time periods between one and eight days and correlated with lags of \pm 5 timesteps. In the AN as well as the ITCZ area a feedback exists between SST and mechanical mixing, indicated by the symmetric form of the cross-correlation function. Enhanced (reduced) mixing leads to a cold (warm) SST anomaly, and a warm (cold) SST anomaly reversly leads to less (more) mechanical mixing. We hypothesize that the latter is due to the increased (decreased) static stability of the upper ocean temperature profile. The lead-lag correlation peaks at zero lag, but at single day integration it slants towards SST evolution leading ocean mechanical mixing. This is consistent with the direct influence of the static stability on ocean vertical mixing. Integrating over several days leads to a curve slanted towards the mechanical mixing leading the SST evolution. In this case, the increased mixing results in 

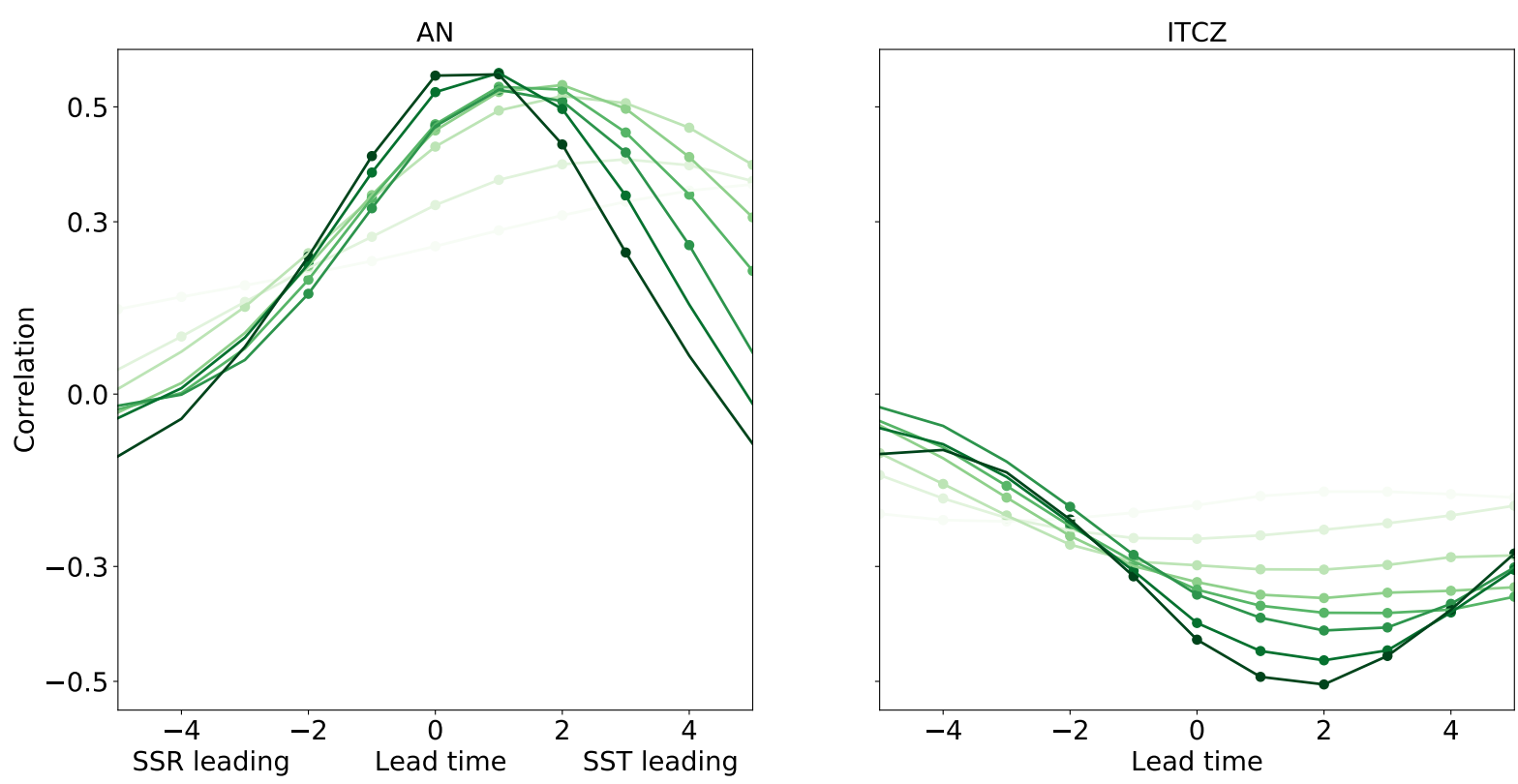

Figure 5.5: Lead-lag cross-correlation between sea surface temperature and downward solar radiation. Line shading and marking as in Fig. 5.4.

surface cooling by entraining cold water from below the mixed layer into it. Integration over a longer period of time yields a nearly symmetrical curve in which the reciprocal influence of both terms on each other is reflected. The coefficient of determination, $R^{2}$, for SST evolution dependent on vertical ocean mixing is 0.36 . Hence, the mixing process may explain roughly $35 \%$ of SST variance in the region.

Now we turn to the atmospheric component of the feedback loop. Lead-lag correlations between SST and surface solar irradiance demonstrate the presence of additional coupled ocean-atmosphere feedbacks (Figure 5.5). In the AN region a positive surface radiation anomaly is accompanied by a positive SST anomaly at all leads and lags. Shallow stratoculumulus clouds are the dominant cloud type in this region. A positive SST anomaly can reduce such cloud cover by destabilising the atmosphere (Clement et al., 2009; Bellomo et al., 2015; Klein and Hartmann, 1993). This allows for more shortwave radiation to reach the surface and thereby reinforces the initial SST anomaly. At all integration timescales the correlation curve is slanted towards SST leading surface radiation. This indicates the dominant role of the ocean driving cloud cover changes. Surface radiation leading SST variations also yields significant correlations when the integration time is large enough for the excessive radiation to warm the shallow mixed layer (7-8 days). This positive coupled feedback includes the ocean mixing component (Figure 5.4) which further reinforces the SST anomaly. The results indicate that the upper ocean column is stabilised by surface warming, which reduces ocean vertical mixing.

In contrast to the AN region, $\mathrm{SST}$ and surface irradiance are negatively correlated in the ITCZ region. The dominant cloud type in this region are (deep) convective clouds. A 
positive SST anomaly triggers convection in the atmosphere, leading to more cloud cover and less surface irradiance. Stability of the upper ocean decreases, allowing for more effective vertical ocean mixing to take place. In this case, the coupled feedback restores the SST anomaly. The slant of the correlation curve towards SST leading surface radiation anomalies shows the dominant influence of SST on surface radiation. Correlations in which surface radiation lead SST anomalies are lower than SST leading surface radiation, but remain significant.

\subsection{Discussion}

Using in-situ observations, satellite based and reanalysis data, we present evidence for a coupled air-sea feedback in the tropical Atlantic. An essential link in the feedback loop involves upper ocean turbulent mixing in response to radiation changes associated with cloud cover variability. SST anomalies are reinforced in the region off the coast of Angola/Namibia where shallow stratocumulus type clouds dominate. A positive SST anomaly destabilises the atmospheric boundary layer. This leads to reduced cloud cover and more radiation reaching the surface, which enhances the SST anomaly. We find an additional reinforcing mechanism that consitutes of decreased mechanical vertical mixing due to the stabilised upper ocean column caused by the postive SST anomaly. In deep convective regions positive SST anomalies are damped as they reinforce deep atmospheric convection which leads to more cloud cover and reduction of surface radiation. The upper ocean static stability decreases and more mechanical mixing takes place leading to further damping of the SST anomaly. The vertical ocean mixing induced by the feedback explains $35 \%$ of SST variance in the region.

The role of vertical mixing in the upper ocean heat budget has been shown before for the tropical Atlantic (e.g. Foltz et al. (2013, 2018); Hummels et al. (2014)), but its relation to atmospheric variability as part of a coupled feed back has not been shown in this way. The feedback operates on short time scales of several days to weeks, but its influence can magnify interannual SST variability as well. Correlations between SST and the vertical temperature gradient are as high as 0.92 without lag, and persist at time lags of months (not shown). In the AN box, where the feedback reinforces the anomaly, SST interannual variance is considerably larger than in the ITCZ box where it restores the anomaly.

High temporal resolution ocean and surface flux data could further quantify the oceanic part of the feedback loop found here. The PIRATA data streams are excellent sources of data for that purpose. The current observational data record shows clear evidence, but there is not enough high resolution data to estimate all terms. Also, the amplitude of the mixing could only be estimated from a simple bulk formulation. However, single column coupled ocean-atmosphere models confirm the essential role of oceanic vertical mixing and vertical stability in this region (Hartung et al., 2018, and Chapter 3). The latter study 
investigates the impact of vertical mixing at a location inside the AN box. In this study, it is shown that incorrect model stability contributes to the SST bias. However, the coupled ocean mixing - cloud feedback could not be found. The case used in Chapter 3 featured mainly shallow convective clouds rather than stratoculumulus. An analysis with a coupled ocean-atmosphere SCM should be repeated at a location with confirmed stratoculumulus cloud cover, to corroborate the mechanism proposed in this study.

Recently, many studies address Tropical Atlantic variability and the respective biases in coupled global climate models (Deppenmeier et al., 2016; Ding et al., 2015; Richter et al., 2014a; Voldoire et al., 2014; Richter et al., 2013, 2012; Breugem et al., 2006). The main focus has been on large scale processes and feedbacks. Our study highlights the importance of local, short time scale oceanic and atmospheric boundary layer processes to tropical Atlantic variability. Correct representation of this coupled air-sea feedback in numerical weather prediction and climate models can improve reliability of weather and seasonal forecasts and climate projections. 

Chapter 6

Synthesis and Recommendations 


\subsection{Overview of the present research}

Tropical Atlantic climate is governed by periodically recurring patterns of sea surface temperature (SST) variability. In boreal spring, temperatures along the equator are uniformly higher than $27^{\circ} \mathrm{C}$, and near-equatorial meridional and zonal SST gradients are very small. In late spring, the eastern tropical Atlantic cools rapidly. A cold tongue develops on the eastern equator, which gives rise to a zonal SST gradient of cold water in the east and warmer water in the west. The near-equatorial meridional temperature gradient also increases, which causes the inter-tropical convergence zone (ITCZ) to move towards the north. The equatorial cold tongue receides in boreal fall. Eastern equatorial SST gradually rise, until they reach their maximum again in spring. The seasonal cycle of SST is stronger in some years than in others, giving rise to a pattern of inter-annual variability that closely resembles the pattern of the annual cycle.

Particularly in the tropics, SSTs impact the large scale atmospheric circulation, precipitation, and cloud cover. SST anomalies in the tropical Atlantic can assert influence on regions as far away as Europe and North America. In the closer vicinity, tropical Atlantic SST influence the West African monsoon and rainfall and drought in South America. On both continents, local populations depend on water supplied by precipitation. They suffer large agricultural losses when the expected rain arrives late. In a warming climate, the seasons may change, and along with them the timing, intensity, and length of rainfall and drought periods. Reliable forecasts of these events are desirable, but can only be achieved when we understand the processes that cause SST variability in the tropical Atlantic, and are able to accurately and reliably simulate them in the numerical models used to perform weather forecasts and climate projections.

In this thesis, we study different aspects of tropical Atlantic climate variability related to periodically recurring SST patterns in the (south-)east of the ocean basin. We use a variety of tools and methods to identify processes, feedbacks, and interactions that give rise to, sustain, and strengthen modes of SST variability. We investigate large scale dynamics from three dimensional fully coupled atmosphere-ocean global climate model (GCM) output, and focus on smaller scales using a coupled atmosphere-ocean single column model (SCM) and observational data. We perform bias development analysis and sensitivity experiments, ultimately reducing the warm SST bias in the south-eastern tropical Atlantic in the numerical climate model EC-Earth.

In the following section, we summarise our findings guided by the research questions formulated in the introduction (chapter 1).

Q1 To what extent does the Bjerknes Feedback contribute to inter-annual SST variability in the Tropical Atlantic? 
The theory that basin wide air-sea interaction gives rise to large parts of sea surface temperature variance in the eastern tropical Atlantic has been put forward early in the literature (Servain, 1991; Ruiz-Barradas et al., 2000). These air-sea interactions form oscillatory modes, which offer the chance to potentially predict SST associated to them in advance (Ding et al., 2010; Brandt et al., 2011). Assessing the mechanism behind the interannual mode of SST variability from observational data, Keenlyside and Latif (2007) conclude that the Bjerknes Feedback is active in the tropical Atlantic, and that it forms a closed feedback loop similar to that associated to the El Niño Southern Oscillation in the Pacific. Several other studies based on simple and complex models and observational estimates point to an active role of the Bjerknes Feedback (Merle, 1980; Hirst and Hastenrath, 1983; Philander, 1986; Servain, 1991; Chang et al., 2000; Ruiz-Barradas et al., 2000). However, other theories to explain eastern tropical Atlantic SST variability have been put forward only several years ago (Richter et al., 2013; Nnamchi et al., 2015). Richter et al. (2013) point out that not all events in which the eastern-equatorial cold tongue forms anomalously can be related to equatorial wind stress anomalies, and Nnamchi et al. (2015) hypothesize that eastern equatorial SST variability could be controlled by thermodynamics alone. The authors of the latter study use a set of slab-ocean experiments to show that SST variability can also be produced in the absence of ocean dynamics. Earlier studies could not find this relationship (Ruiz-Barradas et al., 2000), and a more recent study does not find evidence for this theory, either (Prodhomme et al., 2019).

In chapter 2, we re-visit the role of the Bjerknes Feedback in the tropical Atlantic. We investigate whether the feedback can be identified in a set of state-of-the-art reanalysis data other than the one used by the authors of Keenlyside and Latif (2007). Laglead cross-correlation analysis confirms the presence of the Bjerknes Feedback in boreal summer (May - August). We find that all three components of the feedback are active and contribute to SST variability. The individual components are a) the influence of eastern-equatorial sea surface temperature anomalies on wind stress in the west, b) the eastern-equatorial thermocline anomaly following one month after the western wind stress anomaly, and c) local thermocline anomalies impacting overlying sea surface temperatures. We correlate an index of the leading anomaly grid-point-wise with the variable that responds to the lead anomaly, for example an index of eastern tropical Atlantic SST anomalies to zonal wind stress anomalies in the entire basin. In the case of component c) of the feedback, heat content anomalies are correlated grid point wise with SST anomalies of the same grid point. The emerging spatial patterns contain information about the mechanism of the coupling. Here, we briefly discuss the spatial patterns of the individual components of the Bjerknes Feedback named above as we find them in chapter 2.

a) The largest wind response to eastern equatorial SST anomalies occurs in the western tropical Atlantic, and peaks in March, April, and May. The peak of the correlation coincides with the time and location when the western branch of the ITCZ is located on 
the equator. In June, the ITCZ moves to the north, and along with it the maximum of the wind anomaly correlating to eastern equatorial SST anomalies. The weakening of the zonal winds as a response to a warm SST anomaly seems to be strongest at the location of the ITCZ. When the ITCZ moves northward, meridional winds return to the western equatorial tropical Atlantic, which are less influenced by zonal SST gradients than the zonal winds.

b) The response of eastern equatorial heat content to wind stress anomalies in the western tropical Atlantic is located at the eastern part of the basin on the equator in late spring and early summer. Heat content changes occur due to deepening or shoaling of the thermocline, which separates the upper ocean mixed layer from the stratified deeper ocean. These variations of thermocline depth are propagated by equatorial Kelvin waves, which are excited by wind stress anomalies in the west. The subsurface Kelvin wave is closely locked to the equatorial waveguide and can, therefore, only transmit anomalies along the equator. The ocean dynamic component of the Bjerknes Feedback can be identified in the months when the heat content response to western equatorial zonal wind stress anomalies is maximal along the waveguide, in the east on the equator. This is the case in May and June. In July, the maximum of the wind stress - heat content correlation moves to the south, off the equator. The Kelvin wave travelling along the thermocline on the equator cannot reach this off-equatorial region. This implies a receding influence of the Kelvin wave on eastern-equatorial heat content. The off-equatorial correlation likely stems from dispersing of the equatorial heat content anomaly, originally caused by ocean dynamics. The equatorial ocean dynamics component of the Bjerknes Feedback is no longer active when the response is mainly off-equatorial.

c) Local correlation between heat content and sea surface temperature anomalies closely follow the pattern of component b) of the Bjerknes Feedback. In May and June, the peak of the correlation is located at the eastern part of the basin on the equator, where the western wind stress has preconditioned the thermocline to be shallow and the heat content hence asserts large influence on overlying SST. In July, the peak of the heat content - SST correlation moves south along with the heat content anomaly related to western-equatorial zonal wind stress. The heat content anomaly caused by component b) of the feedback has moved off-equatorial, and with it regions that are likley to display strong sub-surface-surface coupling.

There Bjerknes Feedback had earlier been established in models of the tropical Atlantic, and from reanalysis. Here, we confirm that the Bjerknes Feedback is present from stateof-the-art reanalysis data. We have analysed in which months the different components of the mechanism are active, and found a time lag of one month between the western equatorial wind anomaly and the eastern equatorial heat content anomaly. This opens the possibility of predicting the eastern-equatorial sea surface temperature anomaly one month ahead, when wind anomalies in the west are observed. The potential predictability 
of this mechanism can only be used if the numerical models include it. We therefore turn to the question:

Q2 How accurate is the representation of the Bjerknes Feedback in state-of-the-art coupled global climate models?

To answer this question, we make use of pre-industrial control simulation output from the Coupled Model Intercomparison Project Phase 5 (CMIP5, (Taylor et al., 2012)). We perform the same lead-lag cross correlation analysis for each of the feedback components on the CGCM output, and then compare the CGCM results to those from reanalysis data. We use a novel way to compare the two correlations, which accounts for both the spatial structure of the correlation and its strength. For each component, we plot the model correlation strength in the relevant area on the abscissa and the pattern correlation between the model and reanalysis pattern on the ordinate. A model scores highly in this metric if its correlation between the two variables is close to the observed one, and the pattern correlation agreement close to unity.

Most CGCMs reproduce components a) and b) of the feedback, the response of wind stress to SST anomalies, and the heat content anomaly following the wind stress signal, respectively. The mechanisms are captured best when we introduce a one month time lag between the CGCM simulation results and reanalysis results. The CGCM patterns of the SST - wind stress correlation, for example, very closely resembles that from reanalysis data when we compare the response of the models in July to that in reanalysis in June. This time lag is also evident from the seasonal cycles of SST, zonal wind stress, and heat content, and the seasonal cycles of variance of these variables.

However, the CGCMs struggle to accurately simulate the third part of the feedback, in which heat content anomalies lead to overlying SST anomalies. The simulated feedback loop is not closed. Therefore, the Bjerknes Feedback mechanism in the tropical Atlantic does not allow us to predict eastern-equatorial sea surface temperature anomalies with the current CGCMs, where part of the feedback is missing. We attempt to explain the lack of sub-surface-surface coupling by investigating the sub-suface ocean structure. In observations, the tropical Atlantic thermocline is very shallow in the east; the well mixed surface layer is thin. Its shallow depth makes the mixed layer susceptible to temperature change: cold water entraining from below cools thin mixed layers more than thicker layers at the same upwelling. In our analysis we confirm that the subsurface ocean structure in the CGCMs is misrepresented, which has earlier been pointed out by Richter et al. (2012). Although we link incorrect representation of the upper ocean to the missing last component of the Bjerknes Feedback, we were unable to correlate the biased mean state bias to the feedback strength. Recently, Dippe et al. (2018a) suggested that an improved mean state improves the Bjerknes Feedback using a single model. Voldoire et al. (2019) demonstrate improvement of the Bjerknes Feedback representation with using 
an ensemble of six bias-reduced CGCMs. Possibly, the subsurface biases in the CMIP5 ensemble are too large to detect a correlation between mean state bias and feedback representation among that ensemble. Another recent study investigates the relationship between climatological biases and generated variability in CGCMs (Prodhomme et al., 2019). Prodhomme et al. (2019) find that it is the ability to simulate the seasonal cycle, i.e., the representation of strong summer-time cooling in the eastern-equatorial Atlantic, rather than the climatological mean state which is related to realistic variability.

In state-of-the-art-GCMs, the seasonal cycle of sea surface temperature is not only delayed, but also much weaker than in observations. Flawed ocean simulation in the eastern tropical Atlantic prevents the ocean from cooling appropriately, and inhibits the Bjerknes Feedback. This hampers efforts to reliably predict eastern-equatorial SST anomalies. The ensemble of CGCMs with reduced SST bias from (Voldoire et al., 2019) shows slightly improved prediction skill in the easten tropical Atlantic (not shown). However, this result is based on a relatively small ensemble size with a small forecast ensemble, and should be tested for statistical significance in a larger ensemble.

Figure 6.1: Schematic of the Bjerknes Feedback as found in reanalysis data. The two components linking wind stress to SST anomalies and heat content anomalies to wind stress are reproduced by most CMIP5 models, but the sub-surface-surface coupling linking heat content anomalies to SST anomalies on the eastern equator is lacking.

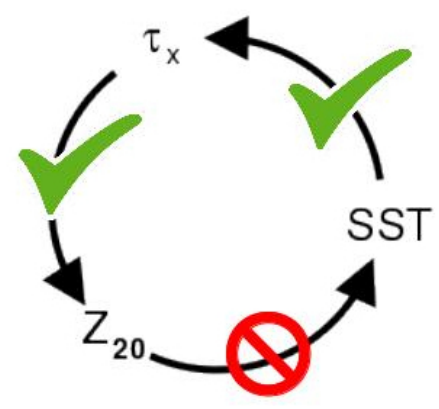

The CGCMs ability to reproduce the seasonal cycle, and especially the summer-time cooling in the eastern tropical Atlantic, emerges to be an important factor for prediction and production of realistic SST variability. Almost all CMIP5 models display large biases and lags in their seasonal cycles, and large biases in their sub-surface-surface coupling (Fig. 6.1). One might well be related to the other. In this thesis, we investigate this common feature guided by the research question:

Q3 What controls the model deficiencies of the sea surface temperature seasonal cycle?

The basin wide dynamics of Bjerknes Feedback are reasonably simulated by CGCMs, but the local part coupling sub-suface and SST is lacking. To address this problem, we perform simulations with a single column model (SCM) version of a state-of-the-art 
coupled global climate model. The set-up of the coupled atmosphere-ocean SCM lets us focus on small-scale vertical processes, and their effect on the ocean reprentation.

In chapter 3, we first test the SCM's ability to simulate the strong observed summer time cooling. The SCM, initialised from observed state at a PIRATA mooring site, reproduces observed SSTs well during the first five days of the simulation starting in June. After that initial phase, the strong SST cooling cannot be reproduced and the SCM builds up a warm SST bias very similar to that of the three dimensional version of the model. A third of the warm bias in the SCM can be removed when the warm, moist near surface bias in the atmosphere is removed. This is in line with a study from (Hourdin et al., 2015), in which the authors find that near surface relative humidity assert large influences in the SST bias. However, a sizeable warm bias remains, even when the SCM is forced with temperature, moisture, and wind from reanalysis data. Another process, intrinsic to the ocean model, must be responsible for this part of the bias. In the absence of large scale ocean dynamics, which are shown to play a minor role at the specific site where the SCM is located (Scannell and McPhaden, 2018), it can only be ocean vertical mixing that insufficiently cools the surface layer. The importance of vertical mixing has been pointed out by many other studies before (Hazeleger and Haarsma, 2005; Hummels et al., 2014; Polo et al., 2015; Planton et al., 2018). Indeed, when we increase the ocean vertical mixing efficiency to a value within the observationally constrained range, the SST bias is reduced to a quarter of its original size. The enhanced ocean vertical mixing enables the SCM to follow observed summer time cooling at the location of the buoy.

Vertical mixing cannot be resolved in CGCMs, due to its small spatial and short temporal scale, its effects on tracers and dynamics are included via parameterisation. The equations representing the parametrised process have to be fitted to observations, which are sparse for turbulent processes. This makes the vertical mixing representation in climate model a source of uncertainty. A campaign in which modelling and observation communities work closely together would be desirable for the purpose of improving model representation of turbulence. However, turbulence is very variable, and such a campaign would therefore have to be wide spread and long lasting.

We use our result from the successful enhanced vertical mixing SCM simulation in the fully coupled global climate model version, to answer the question:

Q4 What is the impact of enhanced turbulent upper ocean mixing on the simulation of the tropical Atlantic climate system?

In chapter 4 we confirm that parameterised processes contribute to the build-up of the warm SST bias in a three dimensional coupled global climate model. We perform heat budget analyses on seasonal hindcast experiments performed with the state-of-the-art CGCM EC-Earth. These experiments are initialised from estimates of the observed state 
of the ocean and atmosphere, and evolve freely from there. By comparing the model simulation output to observations, and how they differ from each other, we study the bias development, rather than the fully developed bias. This allows us to narrow down the processes that cause the bias in the first place. For the heat budget analysis, we choose two areas displaying rapid bias development, one below the summer ITCZ and one in the south-eastern tropical Atlantic. The latter region comprises the buoy location on which the analysis in chapter 2 is based. In both regions subgrid-scale processes are the dominant contributions to the warm SST bias build up. Building on chapter 3, in which we show that the bias can be alleviated completely by enhancing the ocean vertical mixing efficiency, we perform a three dimensional fully coupled global model simulation with EC-Earth with enhanced vertical mixing. We choose a larger numerical value for the mixing efficiency in the CGCM simulation. A value near to the ideal SCM mixing efficiency has been tested in short seasonal hindcast experiments (Exarchou et al., 2017), albeit in combination with other ocean parameterisation changes. The analysis in the latter study remains brief. The authors show that using the optimal SCM value reduces the bias slightly, but a considerable part of it remains. In our study, we strongly increase the mixing efficiency to test the effects of a high mixing scenario.

Enhancing the ocean vertical mixing efficiency reduces the coupled global climate model SST bias, locally by up to a third of its original value. This is a good step towards eliminating the bias, but it is less of an improvement than observed in the single column model (SCM) in chapter 3, where the bias could be eliminated almost entirely. In the SCM, the vertical mixing parameterisation is the main factor determining the SST bias (see chapter 3 Fig. 3.14). At optimal value for $C_{d i f f}$, the SST bias depends very little on the atmospheric bias. The atmosphere in the SCM, however, is forced with temperature, moisture and momentum advection from ERA-Interim, which reduces the atmospheric biases compared to the ones in the 3D model. Potentially, the SCM SST bias would remain larger when forcing the SCM with advection from EC-Earth rather than from ERA-Interim. Testing the $C_{\text {diff }}$ sensitivity to the applied atmospheric forcing could be a next step within this line of research. Moreover, there is no (erroneous) temperature advection in the ocean in the SCM. While we show that ocean advection plays a minor role in the CGCM bias development (see chapter 5 Fig. 4.5), this result is based on short seasonal hindcasts. Advection might contribute more substantially to the bias in an equilibrated situation, where remote oceanic biases are fully developped. These biases can be advected into the regions we analyse, and decrease the beneficial effect of the enhanced mixing parameterisation.

Enhanced mixing also improves the atmospheric circulation in present climate, associated to improved present day SST. Precipitation connected to the ITCZ increases beneficially, and wind biases are reduced. The simulation is improved in the atmosphere and in the upper ocean mixed layer. At the bottom of the mixed layer, however, the ocean is warmed in the enhanced mixing experiment compared to the control experiment. Heat from the 
surface is transported downward in the mixed layer and accumulates at the bottom of it. Here the mixed layer is separated from the deeper ocean by the thermocline, across which heat is not readily mixed, even in the enhanced mixing scenario. Heat accumulates at the bottom of the mixed layer in both regions, the south-eatern tropical Atlantic and below the summer ITCZ. In the ITCZ box, however, the water column also warms below the mixed layer. Here, we see an effect of advection of warmer water from remote areas along isopycnals.

We continue both the control and the sensitivity experiment in climate projection mode following the representative concentration pathway 8.5 until the end of the 21st century, focusing our analysis on the tropical Atlantic. The default version of EC-Earth projects a warming of $4.5^{\circ} \mathrm{C}$, an increase in tropical maritime precipitation, and weakening of the surface winds at the end of the century. The enhanced mixing parameterisation strongly impacts the climate change signal in the tropical Atlantic. At the end of the century, when the ocean vertical mixing efficiency is increased, the ITCZ is stronger and narrower. In spring, the ITCZ moves towards the northern hemisphere, more so than in the control experiment, and in summer and fall rainfall remains tightly locked on the equator, displaying the narrowing of the ITCZ. This is in line with the sea surface temperature change differences. In the enhanced mixing projection, SSTs are higher south of the equator from June to November than in the control, moving the ITCZ southward.

We demonstrate that the climate change signal in global coupled climate models is strongly dependent on the ocean vertical mixing parameterisation. This highlights the important accurately representing this process in projections of future climate. Collaborations between modelers and observationalists is necessary to improve the current model representation of ocean vertical mixing. A combined and iterative effort of observing and modeling the system could take the scientific community closer to better climate model solutions, and thereby close to producing reliable projections.

Ocean vertical mixing asserts a large influence on not only the ocean, but also the atmosphere, and the process is not accurately represented in state-of-the-art CGCMs. We wonder what influences the strength of vertical mixing. Therefore, we return to in-situ observations and reanalysis data to study the interaction of turbulent processes with the climate system. In chapter 5 we ask the question:

Q5 Is turbulent ocean mixing impacted by ocean interior processes alone, or if not, to what extent does the atmosphere assert (local) influence?

In chapter 5 we focus on the effect of SST and surface shortwave radiation (SSR) on vertical mixing. In the heat budget in chapter 4, which describes the different contributions leading to the warm SST bias, we observe an increasing warming by the subgrid-scale 
processes with time. Simultaneously, the SST bias increases, and upper ocean stratification becomes stronger in the model than in reanalysis. Our analysis shows that surface fluxes dampen the warm bias, rather than cause it. In the first few days after the initialisation SSR is even underestimated, rather than excessive. After a warm SST bias has developed within a couple of days, the SSR bias at the surface switches sign, and is now overestimated. These results from the model suggest some relationship between the warm SST bias and the SSR bias, that places the SST bias in the driving position, rather than responding to SSR. We use in-situ and reanalysis data in lagged correlation analysis to investigate whether there is a connection between the upper ocean vertical mixing, ocean vertical stratification, SST, and surface solar radiation. We focus again on the two regions we have singled out before, one below the ITCZ and one in the south-eastern tropical Atlantic.

We cross-correlate (parameterised) ocean vertical mixing with SST, and SST with surface shortwave radiation with different lag-times, and find significant negative correlations between mixing and SST in both regions, and SST and SSR correlations of different sign depending on the region. Different timescales of our correlation analysis reveal different processes. The lead-lag correlation function between ocean mixing and SST changes sign depending on the timescales we investigate in both regions. At daily resolution the curve is slanted towards SST leading the vertical mixing. We argue that this could be due to the stability influence on vertical mixing: increased SST leads to increased vertical ocean stability, which suppresses vertical mixing. When we correlate ocean vertical mixing and SST on longer timescales, such as 5 days, the correlation function slants towards vertical mixing leading SST. Sustained mixing restores the original SST anomaly. Correlating at longer timescales of up to 8 days leads to a symmetric lead lag cross correlation function, indicative of a feedback between the two variables. Combined with the cross correlation function between SST and SSR we argue that there is a feedback loop between ocean mixing, SST and cloud cover (of which SSR is indicative). The sign of the correlation between SST and SSR depends on the region, it is positive in the south-eastern tropical Atlantic, where warm SST anomalies lead to increased SSR by reducing stratocumulus cloud cover via decreased atmospheric stability, and negative below the ITCZ where SSR decreases with a warm SST anomaly, by triggering deep convection above the warm anomaly. The feedback loop in which short time-scale SST anomalies (and hence ocean vertical stability) affect vertical mixing and SSR is positive in the south-eastern tropical Atlantic, increasing the warm anomaly, and negative below the ITCZ, where the anomaly is restored.

The lead-lag correlation analysis in this chapter hints to such a feedback loop, but cannot conclusively prove it. We argue that wind variability alone does not explain the variability of the mixed layer depth, which is modulated by vertical mixing. Mechanistic studies of this process using models, such as the SCM used in chapter 3, could be performed to test this feedback. In chapter 3, the experiment replacing ocean stability showed increased 
vertical mixing, and a reduced SST bias, hinting that ocean stability indeed impacts vertical mixing. Detailed observational studies using long time series of high temporal resolution observations of the subsurface ocean and surface fluxes including momentum and heat transfer are needed to further investigate the feedback. Information about the cloud cover is also desirable, to corroborate the effect and its dependence on the cloud type. Such information can best be obtained from vertical atmosphere profiles, which are rarely available above the open ocean.

\subsection{Recommendations}

In this thesis, we investigate processes that lead to SST variability in the tropical Atlantic, and how they relate to model biases. Using different methods, data, and models, we are able to look at the tropical Atlantic from multiple angles. This allowed us to shed light on several different enigmas of tropical Atlantic variability and its representation in models, it also means that many alternative paths for research presented themselves. Here, we outline possible avenues of investigation for future research.

\subsubsection{Single Column Model Studies}

Upper ocean vertical mixing plays a dominant role in determining the summer time cooling in the (south-) eastern tropical Atlantic (Scannell and McPhaden, 2018; Planton et al., 2018), and is underrepresented in model simulations (Hazeleger and Haarsma, 2005). In chapter 3 we show that increasing the ocean vertical mixing efficiency alone can almost entirely eliminate the warm SST bias in the south-eastern tropical Atlantic. Mixing processes are a source of uncertainty in climate model simulations, since they cannot explicitly be resolved in climate models - their effect always has to be parameterised. To test the robustness of reducing the SST bias by increasing vertical mixing, the SCM could be placed elsewhere at different locations in the tropical Atlantic cold tongue, and the same suite of vertical mixing sensitivity experiments as in chapter 3 could be performed. At locations and times where observations such as the PIRATA buoy ones are not available, larger temporal and spatial sampling could also give insights into the system sensitivity to vertical mixing.

Furthermore, there is a clear need for more extensive observations to constrain the parameterisations. These observations would have to be of very high temporal resolution, and ideally be representative of different meteorological conditions. This is most likely achievable with point-observations, ship-based or from moorings. As demonstrated in chapter 3, the coupled ocean-atmosphere single column model is a convenient tool for those situations, in which we can test model performance agains observations. The SCM could be situated at locations where turbulence measurements, such as the ones obtained 
in (Schafstall et al., 2010), are available. This would allow estimates of which changes have to be made in the parameterisation scheme to accurately represent vertical mixing, rather than inferring the vertical mixing needed for the correct SST evolution.

The single column model can also shed light on the influence of the large scale circulation on vertical mixing. In chapter 3, we use the SCM without horizontal ocean advection at the boundaries. This likely reduces vertical mixing, because the source term of vertical shear is lacking. The parameter sweep in chapter 3 could be repeated with (varying) horizontal advection in the ocean, to estimate the impact of shear on the vertical mixing.

Large scale forcing in the atmosphere also asserts influence on the SST evolution. The effect of varying large-scale forcing on the column could be tested in either idealised settings, or in case studies where times with, for example, strong wind forcing are selected and compared to cases with weaker wind forcing. Similarly, cases of large moisture advection could be compared to cases with less moisture advection. In idealised experiments, the large-scale forcing could be altered to represent a certain state of the atmosphere. For example, a continuos deck of stratocumulus clouds could be advected into the model column, to test the effect of cloud types on the feedback in chapter 5. Furthermore, as mentioned above the SCM could be forced with data from its $3 \mathrm{D}$ counterpart, to investigate the effect of the CGCM atmospheric bias on the ocean, and on parameter sensitivity.

Further, there is the possibility of extending and testing the ocean vertical mixing parametrisation. In chapter 3 we hypothesise that the effect of wind bursts are not represented in the current ocean vertical mixing parameterisation, and that the vertical eddy coefficients could be too low as a consequence. Wind bursts could lead to highly intermittent periods of strong mixing which could reduce the ocean column stability, and thereby increase sustained vertical mixing. To test the impact of mean wind versus high frequency variations in the wind field, differently varying (idealised) momentum forcings could be applied. A different mechanism that is possibly underestimated in the present default mixing parameterisation is the effect of Langmuir Cells. In chapter 3 we show that increasing the Langmuir parameter asserts little effect on the SST evolution, but the present day parameterisation of these processes might be incomplete (Belcher et al., 2012). Recently, second order closure schemes for Langmuir parameterisation, including additional Langmuir source terms, have been developed and tested (Harcourt, 2013, 2015). The effect of additional source terms of turbulent kinetic energy on vertical mixing and the SST evolution could be tested in the SCM setting. 


\subsubsection{Coupled Global Climate Model Studies}

It is often hypothesised that a biased mean state will lead to improved forecasts and predictions, but few studies have proven such a link for the tropical Atlantic (Stockdale et al., 2006). In the framework of (Voldoire et al., 2019), where a multi-model ensemble of bias-reduced seasonal hindcasts has been created, the SST forecast skill was briefly analysed. The bias reduced simulations showed skill improvement for the eastern equatorial Atlantic (ATL3, $20^{\circ} \mathrm{W}-0^{\circ} \mathrm{E}, 3^{\circ} \mathrm{S}-3^{\circ} \mathrm{N}$ ) especially at four months lead time. This result is promising, but has been obtained from a short timeseries (10 years) and a small ensemble. The connection between mean state bias and prediction skill should be investigated in more detail. If a robust link between mean bias and prediction skill emerges, bias correction techniques and ensemble calibration such as in (Krikken et al., 2016) could be applied to test whether prediction skill can be improved.

\section{Multi Model Coordinated Sensitivity Studies}

We have learned that the sea surface temperature seasonal cycle is too weak in most CMIP5 models (chapter 2). While CMIP5 studies can shed light on model trends and deficiencies, it is diffcult to determine mechanisms and causalities with multi model ensemble analyses. Mechanisms can be investigated with process studies and sensitivity experiments. Ideally, these are carried out with not only one CGCM, but coordinated among different groups, so that the outcome can be compared among different models. A good example for this approach is the recent work by (Voldoire et al., 2019). The authors adopt a protocol to alleviate the wind stress bias in six CGCMs, and assess the impact of the improved momentum flux into the ocean on the SST bias. Following this example, the enhanced vertical mixing study in chapter 4 could be repeated in a coordinated sensitivity experiment setting to corroborate the impact of vertical mixing in multiple models and along different mixing parameterisation schemes. Ideally, a multi-model single column model study testing the impact of enhanced vertical mixing parameterisation on the different models (such as presented in chapter 3) would precede this coordinated coupled model effort. Further, high temporal resolution output from the seasonal hindcast experiments with EC-Earth allowed us to trace the bias development to before the emergence of a posititive shortwave radiation bias. This bias and its connection to the emerging warm SST bias could be compared among different models if a suite of experiments with high temporal resolution output was performed. Also, while we do not link the initialisation of the bias to excessive shortwave radiation, neither in the three dimensional nor in the single column model case, a coordinated experiment with prescribed surface short wave radiation forcing could corroborate the notion that SSR does not cause the bias, but might maintain and increase it. Similar studies with the other surface fluxes, especially latent heat, are also interesting to determine their effect on the SST bias. 


\subsubsection{Analysis from Observations}

From lead-lag cross correlation analysis we find indication for a feedback between cloud cover, solar radiation and ocean vertical mixing (chapter 5). The feedback acts to enhance SST anomalies in the south-east, and dampen them below the ITCZ. While lead-lag correlation analysis indicate causality, assumptions made in this chapter could be addressed in further research. In the turbulent kinetic energy equation, stratification acts to reduce vertical mixing. Observations in situations with varying levels of stratification should be identified and analysed for the causality. Similarly as proposed for the SCM studies, cases of observational records with differing cloud properties could be selected and analysed for the ocean mixing - SST - SSR feedback.

\subsection{Closing remarks}

Two areas with large sea surface temperature biases are identified in this thesis: the eastern equatorial cold tongue, and the south-eastern tropical Atlantic. Both regions feature a shallow mixed layer, which makes them susceptible to air-sea interaction. In future analysis, the south-eastern Atlantic region could be subdivided into off-coast and near-coast regions. In the heat budget analysis (chapters 4 and 5) we have analysed a relatively large area which likely comprises parts of both, but for an in-depth study it would be advisable to keep them apart. Cloud properties, for example, rapidly change with distance from the coast, and therefore locations with different cloud characteristics should be investigated separately. On the ocean side, along shore surface winds cause coastal upwelling only in a very thin strip along the coast (Small et al., 2014).

There are many more avenues to explore, from time-dependent variation of the Bjerknes Feedback in CMIP5 models, to detailed analysis with the observation - SCM combination. In this thesis we have employed a number of the possible tools to shed light on the complex system of the tropical Atlantic. We have learned that coupled global climate models capture the basin wide dynamics of the Bjerknes Feedback, but that errors in the ocean model hamper subsurface-surface ocean interaction that is observed in the natural world. As a consequence, surface cooling in the eastern tropical Atlantic is too weak compared to observations. Deficient vertical mixing in the ocean model could be responsible for this. We show using a coupled single column model as well as a coupled global climate model that sea surface temperature biases are reduced under enhanced ocean vertical mixing. This thesis highlights the importance of ocean vertical mixing, and its representation in climate models. Modelers and observationalists will have to work together to improve the understanding and current parameterisation of this vital process. 


\section{References}

Axell, L. B.: Wind-driven internal waves and Langmuir circulations in a numerical ocean model of the southern Baltic Sea, Journal of Geophysical Research: Oceans, 107, 2002.

Balmaseda, M. and Anderson, D.: Impact of initialization strategies and observations on seasonal forecast skill, Geophysical Research Letters, 36, 2009.

Balmaseda, M. A., Mogensen, K., and Weaver, A. T.: Evaluation of the ECMWF ocean reanalysis system ORAS4, Quarterly Journal of the Royal Meteorological Society, 139, 1132-1161, 2013.

Balsamo, G., Beljaars, A., Scipal, K., Viterbo, P., van den Hurk, B., Hirschi, M., and Betts, A. K.: A revised hydrology for the ECMWF model: Verification from field site to terrestrial water storage and impact in the Integrated Forecast System, Journal of hydrometeorology, 10, 623-643, 2009.

Belcher, S. E., Grant, A. L., Hanley, K. E., Fox-Kemper, B., Van Roekel, L., Sullivan, P. P., Large, W. G., Brown, A., Hines, A., Calvert, D., et al.: A global perspective on Langmuir turbulence in the ocean surface boundary layer, Geophysical Research Letters, 39, 2012.

Bellenger, H., Guilyardi, É., Leloup, J., Lengaigne, M., and Vialard, J.: ENSO representation in climate models: from CMIP3 to CMIP5, Climate Dynamics, 42, 1999-2018, 2014.

Bellomo, K., Clement, A. C., Mauritsen, T., Rädel, G., and Stevens, B.: The influence of cloud feedbacks on equatorial Atlantic variability, Journal of Climate, 28, 2725-2744, 2015.

Biasutti, M.: Forced Sahel rainfall trends in the CMIP5 archive, Journal of Geophysical Research: Atmospheres, 118, 1613-1623, 2013.

Biasutti, M., Battisti, D. S., and Sarachik, E. S.: The Annual Cycle over the Tropical Atlantic, South America, and Africa, Journal of Climate, 16, 2491-2508, doi: 10.1175/15200442(2003)016;2491:TACOTT ¿2.0.CO;2, 2003.

Biasutti, M., Sobel, A., and Kushnir, Y.: AGCM precipitation biases in the tropical Atlantic, Journal of climate, 19, 935-958, 2006.

Biasutti, M., Held, I. M., Sobel, A. H., and Giannini, A.: SST forcings and Sahel rainfall variability in simulations of the twentieth and twenty-first centuries, Journal of Climate, 21, 3471-3486, 2008.

Bjerknes, J.: ATMOSPHERIC TELECONNECTIONS FROM THE EQUATORIAL PACIFIC, Monthly Weather Review, 97, 163-172, doi: 10.1175/1520-0493(1969)097;0163:ATFTEP ¿2.3.CO;2, 1969.

Blanke, B. and Delecluse, P.: Variability of the tropical Atlantic Ocean simulated by a general circulation model with two different mixed-layer physics, Journal of Physical Oceanography, 23, 1363-1388, 1993.

Bony, S. and Dufresne, J.-L.: Marine boundary layer clouds at the heart of tropical cloud feedback uncertainties in climate models, Geophysical Research Letters, 32, 2005.

Bougeault, P. and Lacarrere, P.: Parameterization of Orography-Induced Turbulence in a Mesobeta-Scale Model, Monthly Weather Review, 117, 1872-1890, 1989. 
Bourlès, B., Lumpkin, R., McPhaden, M. J., Hernandez, F., Nobre, P., Campos, E., Yu, L., Planton, S., Busalacchi, A., Moura, A. D., Servain, J., and Trotte, J.: THE PIRATA PROGRAM, Bulletin of the American Meteorological Society, 89, 1111-1126, doi: 10.1175/2008BAMS2462.1, 2008.

Brandt, P., Funk, A., Hormann, V., Dengler, M., Greatbatch, R. J., and Toole, J. M.: Interannual atmospheric variability forced by the deep equatorial Atlantic Ocean, Nature, 473, 497, 2011.

Brandt, P., Funk, A., Tantet, A., Johns, W. E., and Fischer, J.: The Equatorial Undercurrent in the central Atlantic and its relation to tropical Atlantic variability, Climate dynamics, 43, 2985-2997, 2014.

Breugem, W.-P., Hazeleger, W., and Haarsma, R.: Multimodel study of tropical Atlantic variability and change, Geophysical research letters, 33, 2006.

Breugem, W.-P., Hazeleger, W., and Haarsma, R. J.: Mechanisms of northern tropical Atlantic variability and response to CO2 doubling, Journal of climate, 20, 2691-2705, 2007.

Burls, N., Reason, C., Penven, P., and Philander, S.: Similarities between the tropical Atlantic seasonal cycle and ENSO: An energetics perspective, Journal of Geophysical Research: Oceans, 116, 2011.

Burls, N. J., Reason, C. J. C., Penven, P., and Philander, S. G.: Energetics of the Tropical Atlantic Zonal Mode, Journal of Climate, 25, 7442-7466, doi: 10.1175/JCLI-D-11-00602.1, 2012.

Carton, J. A. and Huang, B.: Warm events in the tropical Atlantic, Journal of Physical Oceanography, 24, 888-903, 1994.

Carton, J. A., Cao, X., Giese, B. S., and Da Silva, A. M.: Decadal and interannual SST variability in the tropical Atlantic Ocean, Journal of Physical Oceanography, 26, 1165-1175, 1996.

Cassou, C., Terray, L., and Phillips, A. S.: Tropical Atlantic influence on European heat waves, Journal of climate, 18, 2805-2811, 2005.

Center, N. G. S. F. and Laboratory, O. E.: Sea-viewing Wide Field-of-view Sensor (SeaWiFS) Ocean Color Data, NASA OB.DAA, 2014.

Chang, C.-Y., Carton, J. A., Grodsky, S. A., and Nigam, S.: Seasonal climate of the tropical Atlantic sector in the NCAR Community Climate System Model 3: Error structure and probable causes of errors, Journal of Climate, 20, 1053-1070, 2007.

Chang, P., Ji, L., and Li, H.: A decadal climate variation in the tropical Atlantic Ocean from thermodynamic air-sea interactions, Nature, 385, 516, 1997.

Chang, P., Saravanan, R., Ji, L., and Hegerl, G. C.: The effect of local sea surface temperatures on atmospheric circulation over the tropical Atlantic sector, Journal of Climate, 13, 2195-2216, 2000.

Chang, P., Yamagata, T., Schopf, P., Behera, S., Carton, J., Kessler, W., Meyers, G., Qu, T., Schott, F., Shetye, S., et al.: Climate fluctuations of tropical coupled systems - the role of ocean dynamics, Journal of Climate, 19, 5122-5174, 2006.

Clement, A. C., Burgman, R., and Norris, J. R.: Observational and model evidence for positive low-level cloud feedback, Science, 325, 460-464, 2009.

CMIP5 Coupled Model Intercomparison Project, W. W.: URL http://cmip-pcmdi.llnl.gov/cmip5/availability.html, Accessed: 2015-07-16.

Cook, K. H. and Edward, K. V.: Coupled model simulations of the West African monsoon system: 20th century simulations and 21st century predictions, in: J. Climate, Citeseer, 2006.

Cravatte, S., Kessler, W., Smith, N., Wijffels, S., and Authors, C.: First report of TPOS 2020, Tech. rep., GOOS-215, 200pp, 2016.

Davey, M., Huddleston, M., Sperber, K., Braconnot, P., Bryan, F., Chen, D., Colman, R., Cooper, C., Cubasch, U., Delecluse, P., et al.: STOIC: a study of coupled model climatology and variability in 
tropical ocean regions, Climate Dynamics, 18, 403-420, 2002.

Deckert, R. and Dameris, M.: Higher tropical SSTs strengthen the tropical upwelling via deep convection, Geophysical Research Letters, 35, 2008.

Dee, D., Uppala, S., Simmons, A., Berrisford, P., Poli, P., Kobayashi, S., Andrae, U., Balmaseda, M., Balsamo, G., Bauer, P., et al.: The ERA-Interim reanalysis: Configuration and performance of the data assimilation system, Quarterly Journal of the royal meteorological society, 137, 553-597, 2011.

Deppenmeier, A.-L., Haarsma, R. J., and Hazeleger, W.: The Bjerknes feedback in the tropical Atlantic in CMIP5 models, Climate Dynamics, pp. 1-17, 2016.

Deppenmeier, A.-L., Haarsma, R. J., LeSager, P., and Hazeleger, W.: The effect of vertical ocean mixing efficiency on the tropical Atlantic in a coupled global climate model., under review at Climate Dynamics.

Deppenmeier, A.-L., Haarsma, R. J., Heerwaarden, C. v., and Hazeleger, W.: The South-Eastern Tropical Atlantic SST bias investigated with a coupled atmosphere-ocean single column model at a PIRATA mooring site., under review at Journal of Climate.

Deser, C., Capotondi, A., Saravanan, R., and Phillips, A. S.: Tropical Pacific and Atlantic Climate Variability in CCSM3, Journal of Climate, 19, 2451-2481, doi: 10.1175/JCLI3759.1, 2006.

DeWitt, D. G.: Diagnosis of the tropical Atlantic near-equatorial SST bias in a directly coupled atmosphere-ocean general circulation model, Geophysical research letters, 32, 2005.

Ding, H., Keenlyside, N. S., and Latif, M.: Seasonal cycle in the upper equatorial Atlantic Ocean, Journal of Geophysical Research: Oceans, 114, 2009.

Ding, H., Keenlyside, N. S., and Latif, M.: Equatorial Atlantic interannual variability: Role of heat content, Journal of Geophysical Research: Oceans, 115, 2010.

Ding, H., Keenlyside, N., Latif, M., Park, W., and Wahl, S.: The impact of mean state errors on equatorial Atlantic interannual variability in a climate model, Journal of Geophysical Research: Oceans, 120, 1133-1151, 2015.

Dippe, T., Greatbatch, R. J., and Ding, H.: On the relationship between Atlantic Niño variability and ocean dynamics, Climate dynamics, pp. 1-16, 2018a.

Dippe, T., Krebs, M., Harlaß, J., and Lübbecke, J. F.: Can climate models simulate the observed strong summer surface cooling in the equatorial Atlantic?, YOUMARES Conference paper, 8, $2018 \mathrm{~b}$.

Doi, T., Vecchi, G. A., Rosati, A. J., and Delworth, T. L.: Biases in the Atlantic ITCZ in seasonalinterannual variations for a coarse-and a high-resolution coupled climate model, Journal of Climate, 25, 5494-5511, 2012.

Eastman, R., Warren, S. G., and Hahn, C. J.: Variations in cloud cover and cloud types over the ocean from surface observations, 1954-2008, Journal of Climate, 24, 5914-5934, 2011.

Exarchou, E., Prodhomme, C., Brodeau, L., Guemas, V., and Doblas-Reyes, F.: Origin of the warm eastern tropical Atlantic SST bias in a climate model, Climate Dynamics, pp. 1-22, 2017.

Fofonoff, N. P. and Millard Jr, R.: Algorithms for the computation of fundamental properties of seawater., 1983.

Foltz, G. R. and McPhaden, M. J.: Interaction between the Atlantic meridional and Niño modes, Geophysical Research Letters, 37, 2010.

Foltz, G. R., Grodsky, S. A., Carton, J. A., and McPhaden, M. J.: Seasonal mixed layer heat budget of the tropical Atlantic Ocean, Journal of Geophysical Research: Oceans (1978-2012), 108, 2003.

Foltz, G. R., Schmid, C., and Lumpkin, R.: Seasonal cycle of the mixed layer heat budget in the 
northeastern tropical Atlantic Ocean, Journal of Climate, 26, 8169-8188, 2013.

Foltz, G. R., Schmid, C., and Lumpkin, R.: An Enhanced PIRATA Dataset for Tropical Atlantic OceanAtmosphere Research, Journal of Climate, 31, 1499-1524, 2018.

Frankignoul, C. and Hasselmann, K.: Stochastic climate models, Part II Application to sea-surface temperature anomalies and thermocline variability, Tellus, 29, 289-305, 1977.

Fu, R., Del Genio, A. D., and Rossow, W. B.: Influence of ocean surface conditions on atmospheric vertical thermodynamic structure and deep convection, Journal of climate, 7, 1092-1108, 1994.

Gadgil, S., Joseph, P., and Joshi, N.: Ocean-atmosphere coupling over monsoon regions, Nature, 312, $141,1984$.

Gaetani, M. and Mohino, E.: Decadal prediction of the Sahelian precipitation in CMIP5 simulations, Journal of Climate, 26, 7708-7719, 2013.

García-Serrano, J., Losada, T., Rodríguez-Fonseca, B., and Polo, I.: Tropical Atlantic variability modes (1979-2002). Part II: Time-evolving atmospheric circulation related to SST-forced tropical convection, Journal of Climate, 21, 6476-6497, 2008.

Gaspar, P., Grégoris, Y., and Lefevre, J.-M.: A simple eddy kinetic energy model for simulations of the oceanic vertical mixing: Tests at station Papa and Long-Term Upper Ocean Study site, Journal of Geophysical Research: Oceans, 95, 16 179-16 193, 1990.

Giannini, A., Saravanan, R., and Chang, P.: Oceanic forcing of Sahel rainfall on interannual to interdecadal time scales, Science, 302, 1027-1030, 2003.

Giannini, A., Saravanan, R., and Chang, P.: The preconditioning role of tropical Atlantic variability in the development of the ENSO teleconnection: implications for the prediction of Nordeste rainfall, Climate Dynamics, 22, 839-855, 2004.

Goubanova, K., Sanchez-Gomez, E., Frauen, C., and Voldoire, A.: Respective roles of remote and local wind stress forcings in the development of warm SST errors in the South-Eastern Tropical Atlantic in a coupled high-resolution model, Climate Dynamics, 52, 1359-1382, doi: 10.1007/s00382-018-4197-0, 2019.

Haarsma, R. J. and Hazeleger, W.: Extratropical atmospheric response to equatorial Atlantic cold tongue anomalies, Journal of climate, 20, 2076-2091, 2007.

Ham, Y.-G., Kug, J.-S., and Park, J.-Y.: Two distinct roles of Atlantic SSTs in ENSO variability: North tropical Atlantic SST and Atlantic Niño, Geophysical Research Letters, 40, 4012-4017, 2013.

Harcourt, R. R.: A second-moment closure model of Langmuir turbulence, Journal of Physical Oceanography, 43, 673-697, 2013.

Harcourt, R. R.: An improved second-moment closure model of Langmuir turbulence, Journal of Physical Oceanography, 45, 84-103, 2015.

Harlaß, J., Latif, M., and Park, W.: Alleviating tropical Atlantic sector biases in the Kiel climate model by enhancing horizontal and vertical atmosphere model resolution: climatology and interannual variability, Climate Dynamics, 50, 2605-2635, 2018.

Hartung, K., Svensson, G., Struthers, H., Deppenmeier, A.-L., and Hazeleger, W.: An EC-Earth coupled atmosphere-ocean single-column model (AOSCM. v1_EC-Earth3) for studying coupled marine and polar processes, Geoscientific Model Development, 11, 4117-4137, 2018.

Hazeleger, W. and Haarsma, R. J.: Sensitivity of tropical Atlantic climate to mixing in a coupled oceanatmosphere model, Climate dynamics, 25, 387-399, 2005.

Hazeleger, W., de Vries, P., and van Oldenborgh, G. J.: Do tropical cells ventilate the Indo-Pacific 
Equatorial Thermocline?, Geophysical research letters, 28, 1763-1766, 2001.

Hazeleger, W., de Vries, P., and Friocourt, Y.: Sources of the Equatorial Undercurrent in the Atlantic in a High-Resolution Ocean Model, Journal of Physical Oceanography, 33, 677-693, doi: 10.1175/15200485(2003)33;677:SOTEUI ¿2.0.CO;2, 2003.

Hazeleger, W., Severijns, C., Semmler, T., ŞŞtefăănescu, S., Yang, S., Wang, X., Wyser, K., Dutra, E., Baldasano, J. M., Bintanja, R., et al.: EC-Earth: A Seamless Earth-System Prediction Approach in Action., Bulletin of the American Meteorological Society, 91, 2010.

Hazeleger, W., Wang, X., Severijns, C., Ştefănescu, S., Bintanja, R., Sterl, A., Wyser, K., Semmler, T., Yang, S., Van den Hurk, B., et al.: EC-Earth V2. 2: description and validation of a new seamless earth system prediction model, Climate Dynamics, 39, 2611-2629, 2012.

Held, I., Delworth, T., Lu, J., Findell, K. u., and Knutson, T.: Simulation of Sahel drought in the 20th and 21st centuries, Proceedings of the National Academy of Sciences, 102, 17 891-17 896, 2005.

Hirst, A. C. and Hastenrath, S.: Atmosphere-ocean mechanisms of climate anomalies in the Angolatropical Atlantic sector, Journal of Physical Oceanography, 13, 1146-1157, 1983.

Hourdin, F., Găinusă-Bogdan, A., Braconnot, P., Dufresne, J.-L., Traore, A.-K., and Rio, C.: Air moisture control on ocean surface temperature, hidden key to the warm bias enigma, Geophysical Research Letters, 42, 10-885, 2015.

Hu, Z.-Z., Huang, B., and Pegion, K.: Low cloud errors over the southeastern Atlantic in the NCEP CFS and their association with lower-tropospheric stability and air-sea interaction, Journal of Geophysical Research: Atmospheres, 113, 2008.

Hu, Z.-Z., Huang, B., Hou, Y.-T., Wang, W., Yang, F., Stan, C., and Schneider, E. K.: Sensitivity of tropical climate to low-level clouds in the NCEP climate forecast system, Climate dynamics, 36, 1795-1811, 2011.

Huang, B., Schopf, P. S., and Shukla, J.: Intrinsic ocean-atmosphere variability of the tropical Atlantic Ocean, Journal of Climate, 17, 2058-2077, 2004.

Huang, B., Hu, Z.-Z., and Jha, B.: Evolution of model systematic errors in the tropical Atlantic basin from coupled climate hindcasts, Climate dynamics, 28, 661-682, 2007.

Hummels, R., Dengler, M., Brandt, P., and Schlundt, M.: Diapycnal heat flux and mixed layer heat budget within the Atlantic Cold Tongue, Climate dynamics, 43, 3179-3199, 2014.

Jansen, M. F., Dommenget, D., and Keenlyside, N.: Tropical atmosphere-ocean interactions in a conceptual framework, Journal of Climate, 22, 550-567, 2009.

Jochum, M., Malanotte-Rizzoli, P., and Busalacchi, A.: Tropical instability waves in the Atlantic Ocean, Ocean Modelling, 7, 145-163, 2004.

Johnson, N. C. and Xie, S.-P.: Changes in the sea surface temperature threshold for tropical convection, Nature Geoscience, 3, 842, 2010.

Keenlyside, N. S. and Latif, M.: Understanding equatorial atlantic interannual variability, Journal of Climate, 20, 131-142, 2007.

Klein, S. A. and Hartmann, D. L.: The seasonal cycle of low stratiform clouds, Journal of Climate, 6, 1587-1606, 1993.

Kolmogorov, A. N.: Equations of turbulent motion in an incompressible fluid, in: Dokl. Akad. Nauk SSSR, vol. 30, pp. 299-303, 1941.

Koseki, S., Keenlyside, N., Demissie, T., Toniazzo, T., Counillon, F., Bethke, I., Ilicak, M., and Shen, M.-L.: Causes of the large warm bias in the Angola-Benguela frontal zone in the Norwegian earth 
system model, Climate Dynamics, 50, 4651-4670, 2018.

Krikken, F., Schmeits, M., Vlot, W., Guemas, V., and Hazeleger, W.: Skill improvement of dynamical seasonal Arctic sea ice forecasts, Geophysical Research Letters, 43, 5124-5132, 2016.

Kucharski, F., Bracco, A., Yoo, J., Tompkins, A., Feudale, L., Ruti, P., and Dell'Aquila, A.: A GillMatsuno-type mechanism explains the tropical Atlantic influence on African and Indian monsoon rainfall, Quarterly Journal of the Royal Meteorological Society, 135, 569-579, 2009.

Kumar, B. P., Vialard, J., Lengaigne, M., Murty, V., and McPhaden, M.: TropFlux: air-sea fluxes for the global tropical oceans - description and evaluation, Climate Dynamics, 38, 1521-1543, 2012.

Kushnir, Y., Seager, R., Ting, M., Naik, N., and Nakamura, J.: Mechanisms of tropical atlantic sst influence on north american precipitation variability*, Journal of Climate, 23, 5610-5628, 2010.

Lamb, P. J., Peppler, R. A., and Hastenrath, S.: Interannual variability in the tropical Atlantic, Nature, $322,238,1986$.

Lancker, K., Deppenmeier, A.-L., Demissie, T., and Schmidt, J. O.: Climate change adaptation and the role of fuel subsidies: An empirical bio-economic modeling study for an artisanal open-access fishery, PloS one, 14.8: e0220433, 2019.

Large, W. and Danabasoglu, G.: Attribution and impacts of upper-ocean biases in CCSM3, Journal of Climate, 19, 2325-2346, 2006.

Li, G. and Xie, S.-P.: Origins of tropical-wide SST biases in CMIP multi-model ensembles, Geophysical Research Letters, 39, 2012.

Lin, J.-L.: The Double-ITCZ Problem in IPCC AR4 Coupled GCMs: Ocean-Atmosphere Feedback Analysis, Journal of Climate, 20, 4497-4525, doi: 10.1175/JCLI4272.1, 2007.

Lübbecke, J. F., Böning, C. W., Keenlyside, N. S., and Xie, S.-P.: On the connection between Benguela and equatorial Atlantic Niños and the role of the South Atlantic Anticyclone, Journal of Geophysical Research: Oceans, 115, 2010.

Lübbecke, J. F., Burls, N. J., Reason, C. J., and McPhaden, M. J.: Variability in the South Atlantic anticyclone and the Atlantic Niño mode, Journal of Climate, 27, 8135-8150, 2014.

Lübbecke, J. F., Rodríguez-Fonseca, B., Richter, I., Martín-Rey, M., Losada, T., Polo, I., and Keenlyside, N. S.: Equatorial Atlantic variability-Modes, mechanisms, and global teleconnections, Wiley Interdisciplinary Reviews: Climate Change, 9, e527, 2018.

Luyten, J., Pedlosky, J., and Stommel, H.: The ventilated thermocline, Journal of Physical Oceanography, 13, 292-309, 1983.

Ma, C.-C., Mechoso, C. R., Robertson, A. W., and Arakawa, A.: Peruvian stratus clouds and the tropical Pacific circulation: A coupled ocean-atmosphere GCM study, Journal of Climate, 9, 1635-1645, 1996a.

Ma, C.-C., Mechoso, C. R., Robertson, A. W., and Arakawa, A.: Peruvian Stratus Clouds and the Tropical Pacific Circulation: A Coupled Ocean-Atmosphere GCM Study, Journal of Climate, 9, 16351645, doi: 10.1175/1520-0442(1996)009¡1635:PSCATT¿2.0.CO;2, 1996b.

Madec, G., Rahier, C., and Chartier, M.: A comparison of two-dimensional elliptic solvers for the barotropic streamfunction in a multilevel OGCM, Ocean Modelling, 78, 1-6, 1988.

Madec, G. et al.: NEMO ocean engine, 2011.

Madec, G. et al.: NEMO ocean engine, 2015.

Mahajan, S., Saravanan, R., and Chang, P.: The role of the wind-evaporation-sea surface temperature (WES) feedback in air-sea coupled tropical variability, Atmospheric Research, 94, 19-36, 2009.

Martín-Rey, M., Rodríguez-Fonseca, B., and Polo, I.: Atlantic opportunities for ENSO prediction, Geo- 
physical Research Letters, 42, 6802-6810, 2015.

Merle, J.: Annual and interannual variability of temperature in the eastern equatorial Atlantic- The hypothesis of an Atlantic El Nino, Oceanologica Acta, 3, 209-220, 1980.

Mitchell, T. P. and Wallace, J. M.: The annual cycle in equatorial convection and sea surface temperature, Journal of Climate, 5, 1140-1156, 1992.

Mohino, E., Janicot, S., and Bader, J.: Sahel rainfall and decadal to multi-decadal sea surface temperature variability, Climate Dynamics, 37, 419-440, doi: 10.1007/s00382-010-0867-2, 2011.

Moum, J. N., Perlin, A., Nash, J. D., and McPhaden, M. J.: Seasonal sea surface cooling in the equatorial Pacific cold tongue controlled by ocean mixing, Nature, 500, 64, 2013.

Moura, A. D. and Shukla, J.: On the dynamics of droughts in northeast Brazil: Observations, theory and numerical experiments with a general circulation model, Journal of the atmospheric sciences, 38 , 2653-2675, 1981.

Muñoz, E., Weijer, W., Grodsky, S. A., Bates, S. C., and Wainer, I.: Mean and variability of the tropical Atlantic Ocean in the CCSM4, Journal of Climate, 25, 4860-4882, 2012.

Niiler, P.: One-dimensional models of the upper ocean, Modelling and Prediction of the Upper Layers of the Ocean EB Kraus, 143-172, 1977.

Nnamchi, H. C., Li, J., Kucharski, F., Kang, I.-S., Keenlyside, N. S., Chang, P., and Farneti, R.: Thermodynamic controls of the Atlantic Nino, Nature communications, 6, 2015.

Nobre, P. and Shukla, J.: Variations of sea surface temperature, wind stress, and rainfall over the tropical Atlantic and South America, Journal of Climate, 9, 2464-2479, 1996.

Okumura, Y. and Xie, S.-P.: Interaction of the atlantic equatorial cold tongue and the african monsoon., 17, 3589-3602, 2004.

Okumura, Y. and Xie, S.-P.: Some Overlooked Features of Tropical Atlantic Climate Leading to a New Nino-Like Phenomenon., Journal of climate, 19, 5859-5874, 2006.

Okumura, Y., Xie, S.-P., Numaguti, A., and Tanimoto, Y.: Tropical Atlantic air-sea interaction and its influence on the NAO, Geophysical Research Letters, 28, 1507-1510, 2001.

Patricola, C. M., Li, M., Xu, Z., Chang, P., Saravanan, R., and Hsieh, J.-S.: An investigation of tropical Atlantic bias in a high-resolution coupled regional climate model, Climate dynamics, 39, 2443-2463, 2012 .

Pedlosky, J.: Eastern boundary ventilation and the structure of the thermocline, Journal of Physical Oceanography, 13, 2038-2044, 1983.

Peter, A.-C., Le Hénaff, M., Du Penhoat, Y., Menkes, C. E., Marin, F., Vialard, J., Caniaux, G., and Lazar, A.: A model study of the seasonal mixed layer heat budget in the equatorial Atlantic, Journal of Geophysical Research: Oceans, 111, 2006.

Pezzi, L. and Cavalcanti, I.: The relative importance of ENSO and tropical Atlantic sea surface temperature anomalies for seasonal precipitation over South America: a numerical study, Climate Dynamics, $17,205-212,2001$.

Philander, S.: Unusual conditions in the tropical Atlantic Ocean in 1984, Nature, 322, 236, 1986.

Planton, Y., Voldoire, A., Giordani, H., and Caniaux, G.: Main processes of the Atlantic cold tongue interannual variability, Climate Dynamics, 50, 1495-1512, 2018.

Polo, I., Rodríguez-Fonseca, B., Losada, T., and García-Serrano, J.: Tropical Atlantic variability modes (1979-2002). Part I: Time-evolving SST modes related to West African rainfall, Journal of Climate, $21,6457-6475,2008$. 
Polo, I., Dong, B. W., and Sutton, R. T.: Changes in tropical Atlantic interannual variability from a substantial weakening of the meridional overturning circulation, Climate dynamics, 41, 2765-2784, 2013.

Polo, I., Lazar, A., Rodriguez-Fonseca, B., and Mignot, J.: Growth and decay of the equatorial Atlantic SST mode by means of closed heat budget in a coupled general circulation model, Frontiers in Earth Science, 3, 37, 2015.

Prodhomme, C., Voldoire, A., Exarchou, E., Deppenmeier, A.-L., García-Serrano, J., and Guemas, V.: How does the seasonal cycle control equatorial Atlantic interannual variability?, Geophysical Research Letters, 46(2), 916-922, 2019.

Riahi, K., Rao, S., Krey, V., Cho, C., Chirkov, V., Fischer, G., Kindermann, G., Nakicenovic, N., and Rafaj, P.: RCP 8.5-A scenario of comparatively high greenhouse gas emissions, Climatic Change, 109, 33, 2011.

Richter, I.: Climate model biases in the eastern tropical oceans: Causes, impacts and ways forward, Wiley Interdisciplinary Reviews: Climate Change, 6, 345-358, 2015.

Richter, I. and Xie, S.-P.: On the origin of equatorial Atlantic biases in coupled general circulation models, Climate Dynamics, 31, 587-598, 2008.

Richter, I., Xie, S.-P., Wittenberg, A. T., and Masumoto, Y.: Tropical Atlantic biases and their relation to surface wind stress and terrestrial precipitation, Climate dynamics, 38, 985-1001, 2012.

Richter, I., Behera, S. K., Masumoto, Y., Taguchi, B., Sasaki, H., and Yamagata, T.: Multiple causes of interannual sea surface temperature variability in the equatorial Atlantic Ocean, Nature Geoscience, 6, 43-47, 2013.

Richter, I., Behera, S. K., Doi, T., Taguchi, B., Masumoto, Y., and Xie, S.-P.: What controls equatorial Atlantic winds in boreal spring?, Climate dynamics, 43, 3091-3104, 2014a.

Richter, I., Xie, S.-P., Behera, S. K., Doi, T., and Masumoto, Y.: Equatorial Atlantic variability and its relation to mean state biases in CMIP5, Climate dynamics, 42, 171-188, 2014b.

Richter, I., Doi, T., Behera, S. K., and Keenlyside, N.: On the link between mean state biases and prediction skill in the tropics: an atmospheric perspective, Climate Dynamics, 50, 3355-3374, 2018.

Rodríguez-Fonseca, B., Janicot, S., Mohino, E., Losada, T., Bader, J., Caminade, C., Chauvin, F., Fontaine, B., García-Serrano, J., Gervois, S., et al.: Interannual and decadal SST-forced responses of the West African monsoon, Atmospheric Science Letters, 12, 67-74, 2011.

Rouault, M., Florenchie, P., Fauchereau, N., and Reason, C. J. C.: South East tropical Atlantic warm events and southern African rainfall, Geophysical Research Letters, 30, n/a-n/a, doi: 10.1029/2002GL014840, 8009, 2003.

Rouault, M., Servain, J., Reason, C., Bourlès, B., Rouault, M., and Fauchereau, N.: Extension of PIRATA in the tropical South-East Atlantic: an initial one-year experiment, African Journal of Marine Science, 31, 63-71, doi: 10.2989/AJMS.2009.31.1.5.776, 2009.

Ruiz-Barradas, A., Carton, J. A., and Nigam, S.: Structure of interannual-to-decadal climate variability in the tropical Atlantic sector, Journal of Climate, 13, 3285-3297, 2000.

Scannell, H. A. and McPhaden, M. J.: Seasonal Mixed Layer Temperature Balance in the Southeastern Tropical Atlantic, Journal of Geophysical Research: Oceans, 123, 5557-5570, 2018.

Schafstall, J., Dengler, M., Brandt, P., and Bange, H.: Tidal-induced mixing and diapycnal nutrient fluxes in the Mauritanian upwelling region, Journal of Geophysical Research: Oceans, 115, 2010.

Seo, H., Jochum, M., Murtugudde, R., and Miller, A. J.: Effect of ocean mesoscale variability on the mean state of tropical Atlantic climate, Geophysical research letters, 33, 2006. 
Servain, J.: Simple climatic indices for the tropical Atlantic Ocean and some applications, Journal of Geophysical Research: Oceans, 96, 15 137-15146, 1991.

Servain, J., Busalacchi, A., McPhaden, M. J., Moura, A. D., et al.: A pilot research moored array in the tropical Atlantic (PIRATA), Bulletin of the American Meteorological Society, 79, 2019, 1998.

Shanahan, T. M., Overpeck, J. T., Anchukaitis, K., Beck, J. W., Cole, J. E., Dettman, D. L., Peck, J. A., Scholz, C. A., and King, J. W.: Atlantic forcing of persistent drought in West Africa, science, 324, 377-380, 2009.

Skyllingstad, E. D. and Denbo, D. W.: An ocean large-eddy simulation of Langmuir circulations and convection in the surface mixed layer, Journal of Geophysical Research: Oceans, 100, 8501-8522, 1995.

Small, R. J., Xie, S.-P., Maloney, E. D., de Szoeke, S. P., and Miyama, T.: Intraseasonal variability in the far-east Pacific: Investigation of the role of air-sea coupling in a regional coupled model, Climate dynamics, 36, 867-890, 2011.

Small, R. J., Bacmeister, J., Bailey, D., Baker, A., Bishop, S., Bryan, F., Caron, J., Dennis, J., Gent, P., Hsu, H.-m., et al.: A new synoptic scale resolving global climate simulation using the Community Earth System Model, Journal of Advances in Modeling Earth Systems, 6, 1065-1094, 2014.

Song, Z., Lee, S.-K., Wang, C., Kirtman, B. P., and Qiao, F.: Contributions of the atmosphere-land and ocean-sea ice model components to the tropical Atlantic SST bias in CESM1, Ocean Modelling, 96, 280-290, 2015.

Sterl, A. and Hazeleger, W.: Coupled variability and air-sea interaction in the South Atlantic Ocean, Climate Dynamics, 21, 559-571, 2003.

Stockdale, T. N., Balmaseda, M. A., and Vidard, A.: Tropical Atlantic SST prediction with coupled ocean-atmosphere GCMs, Journal of climate, 19, 6047-6061, 2006.

Sud, Y., Walker, G., and Lau, K.-M.: Mechanisms regulating sea-surface temperatures and deep convection in the tropics, Geophysical research letters, 26, 1019-1022, 1999.

Sutton, R., Jewson, S., and Rowell, D.: The elements of climate variability in the tropical Atlantic region., Journal of Climate, 13, 2000.

Sutton, R. T. and Hodson, D. L.: Atlantic Ocean forcing of North American and European summer climate, science, 309, 115-118, 2005.

Talley, L. D.: Descriptive physical oceanography: an introduction, Academic press, 2011.

Taylor, K. E., Stouffer, R. J., and Meehl, G. A.: An overview of CMIP5 and the experiment design, Bulletin of the American Meteorological Society, 93, 485-498, 2012.

Tompkins, A. M.: On the relationship between tropical convection and sea surface temperature, Journal of climate, 14, 633-637, 2001.

Toniazzo, T. and Woolnough, S.: Development of warm SST errors in the southern tropical Atlantic in CMIP5 decadal hindcasts, Climate Dynamics, 43, 2889-2913, doi: 10.1007/s00382-013-1691-2, 2014.

Uvo, C. B., Repelli, C. A., Zebiak, S. E., and Kushnir, Y.: The relationships between tropical Pacific and Atlantic SST and northeast Brazil monthly precipitation, Journal of Climate, 11, 551-562, 1998.

Valcke, S.: The OASIS3 coupler: a European climate modelling community software, Geoscientific Model Development, 6, 373-388, 2013.

Vancoppenolle, M., Fichefet, T., Goosse, H., Bouillon, S., Beatty, C. K., and Morales Maqueda, M.: LIM3, an advanced sea-ice model for climate simulation and operational oceanography, Mercator Ocean Q Newslett, 28, 16-21, 2008. 
Vannière, B., Guilyardi, E., Madec, G., Doblas-Reyes, F. J., and Woolnough, S.: Using seasonal hindcasts to understand the origin of the equatorial cold tongue bias in CGCMs and its impact on ENSO, Climate dynamics, 40, 963-981, 2013.

Voldoire, A., Claudon, M., Caniaux, G., Giordani, H., and Roehrig, R.: Are atmospheric biases responsible for the tropical Atlantic SST biases in the CNRM-CM5 coupled model?, Climate dynamics, 43, 2963-2984, 2014.

Voldoire, A., Exarchou, E., Sanchez-Gomez, E., Demissie, T., Deppenmeier, A.-L., Frauen, C., Goubanova, K., Hazeleger, W., Keenlyside, N., Koseki, S., et al.: Role of wind stress in driving SST biases in the Tropical Atlantic, Climate Dynamics, pp. 1-24, 2019.

von Storch, J.-S.: Signatures of air-sea interactions in a coupled atmosphere-ocean GCM, Journal of Climate, 13, 3361-3379, 2000.

Wahl, S., Latif, M., Park, W., and Keenlyside, N.: On the tropical Atlantic SST warm bias in the Kiel Climate Model, Climate Dynamics, 36, 891-906, 2011.

Wang, C., Zhang, L., Lee, S.-K., Wu, L., and Mechoso, C. R.: A global perspective on CMIP5 climate model biases, Nature Climate Change, 4, 201, 2014.

Wang, F. and Chang, P.: A linear stability analysis of coupled tropical Atlantic variability, Journal of Climate, 21, 2421-2436, 2008.

Website, L. N.: URL http://www.kayelaby.npl.co.uk/general_physics/2_7/2_7_9.html, Accessed: 2015-02-23.

Wen, C., Xue, Y., Kumar, A., Behringer, D., and Yu, L.: How do uncertainties in NCEP R2 and CFSR surface fluxes impact tropical ocean simulations?, Climate Dynamics, 49, 3327-3344, 2017.

Xie, S.-P. and Carton, J. A.: Tropical Atlantic variability: Patterns, mechanisms, and impacts, Earth Climate: The Ocean-Atmosphere Interaction, Geophys. Monogr, 147, 121-142, 2004.

Xu, Z., Chang, P., Richter, I., and Tang, G.: Diagnosing southeast tropical Atlantic SST and ocean circulation biases in the CMIP5 ensemble, Climate dynamics, 43, 3123-3145, 2014a.

Xu, Z., Li, M., Patricola, C. M., and Chang, P.: Oceanic origin of southeast tropical Atlantic biases, Climate dynamics, 43, 2915-2930, 2014b.

Yoon, J.-H. and Zeng, N.: An Atlantic influence on Amazon rainfall, Climate Dynamics, 34, 249-264, 2010.

Yu, J.-Y. and Mechoso, C. R.: Links between annual variations of Peruvian stratocumulus clouds and of SST in the eastern equatorial Pacific, Journal of Climate, 12, 3305-3318, 1999.

Zebiak, S. E.: Air-sea interaction in the equatorial Atlantic region, Journal of Climate, 6, 1567-1586, 1993.

Zelle, H., Appeldoorn, G., Burgers, G., and van Oldenborgh, G. J.: The relationship between sea surface temperature and thermocline depth in the eastern equatorial Pacific, Journal of physical oceanography, 34, 643-655, 2004.

Zhang, C.: Large-scale variability of atmospheric deep convection in relation to sea surface temperature in the tropics, Journal of Climate, 6, 1898-1913, 1993.

Zhou, J. and Lau, K.-M.: Principal modes of interannual and decadal variability of summer rainfall over South America, International Journal of Climatology: A Journal of the Royal Meteorological Society, 21, 1623-1644, 2001.

Zuidema, P., Chang, P., Medeiros, B., Kirtman, B. P., Mechoso, R., Schneider, E. K., Toniazzo, T., Richter, I., Small, R. J., Bellomo, K., et al.: Challenges and prospects for reducing coupled climate 
model SST biases in the eastern tropical Atlantic and Pacific oceans: The US CLIVAR Eastern Tropical Oceans Synthesis Working Group, Bulletin of the American Meteorological Society, 97, 2305-2328, 2016. 



\section{Acknowledgements}

On these pages I would like to thank everyone who supported me on my journey.

Ten eerste wil ik graag de mensen bedanken die mij de mogelijkheid hebben gegeven om het onderzoek aan te gaan dat naar dit proefschrift heeft geleid. Bert, Wilco en Rein, bedankt dat jullie de uitdaging aan gingen om een scheikundige als klimaat promovendus aan te nemen.

In het bijzonder wil ik graag mijn promotor Wilco en mijn co-promotor en dagelijkse begeleider Rein bedanken. Wilco, bedankt voor je ideeën en inzichten, en dat je me vaak vanuit mijn detail gerichte werkwijze terug naar het grote geheel bracht. Jouw overzicht over de wetenschappelijke wereld is een inspiratie voor me. Bedankt voor je vertrouwen en de mogelijkheden die je voor mij hebt gecreëerd.

Rein, ik wil je bedanken voor je begeleiding, voor onze discussies over de tropische Atlantische oceaan, maar ook voor je steun gedurende de laatste jaren. Buiten de wetenschappelijke begeleiding om ben je er altijd voor me geweest, ik had dit proefschrift niet zonder jou kunnen afronden. Je hielp me door tegenslagen heen en hebt altijd naar me geluisterd, daar ben ik je enorm dankbaar voor.

Al mijn (oud)collega's op het KNMI wil ik graag bedanken voor de fijne tijd in de Bilt en de prettige werksfeer. Ik heb warme herinneringen aan het KNMI, en dat komt vooral door jullie. In het bijzonder wil ik Emma, Eveline en Jessica noemen. Bedankt voor onze gezellige etentjes, lunch wandelingen, koffie en ijs excursies, en dat jullie grappige, maar ook moeilijke momenten met mij hebben gedeeld. Utrecht werd mijn thuis door jullie. Eveline en Jessica, bedankt dat jullie mijn paranimfen willen zijn, het is zo mooi dat de cirkel nu rond is. Kate, thanks for the fun times bouldering together, they lit up the last couple of months in my PhD. Thanks also for adopting almost all of my plants, and letting me dump half my household at your place.. Karin bedankt voor de mountain bike rides, spelletjes, en leuke lunch pauzes, en dat je zo een fijne collega en vriendin bent.

I would also like to thank the MAQ crew, old and new, for the gezelligheid in Wageningen. Especially the Utrecht borrel group, Imme and Alba, thank you for your support and the fun times!

During my PhD I was lucky enough to spend some time in Barcelona, at the Catalan Institute for Climate Sciences (IC3). I would like to thank the group (who have since 
moved to the Barcelona Supercomputing Center) for their warm welcome and the great time I had there. I would like to thank Chloe, especially, for inviting me and collaborating in a truly inspiring way.

I would also like to thank all the collaborators I met and exchanged ideas with during the PREFACE project meetings and teleconferences.

Meine lieben Göttinger Chaoten, vielen Dank für unzählbare Spieleabende und unbezahlbaren Zusammenhalt, auch lange nachdem ich Göttingen verlassen habe. Im selben Atemzug möchte ich mich bei meinen Freunden aus Bad Pyrmont bedanken, Jenni, Lato, Lukas, und natürlich bei meiner allerdicksten Freundin Flo. Meiner Familie bin ich dankbar für jegliche Unterstützung auf dem Weg. Lauri, du hilfst mir zu sein wie ich bin, weil ich eine gute grosse Schwester für dich sein möchte.

En tot slot bedank ik jou Stev, mijn man, mijn partner, mijn thuis. 


\section{Peer-reviewed publications}

\section{First author publications}

Deppenmeier, A.-L., Haarsma, R. J., and Hazeleger, W.: The Bjerknes feedback in the tropical Atlantic in CMIP5 models, Climate Dynamics, pp. 1-17, 2016

Deppenmeier, A.-L., Haarsma, R. J., Heerwaarden, C. v., and Hazeleger, W.: The SouthEastern Tropical Atlantic SST bias investigated with a coupled atmosphere-ocean single column model at a PIRATA mooring site., under review at Journal of Climate

Deppenmeier, A.-L., Haarsma, R. J., LeSager, P., and Hazeleger, W.: The effect of vertical ocean mixing efficiency on the tropical Atlantic in a coupled global climate model., under review at Climate Dynamics

\section{Co-author publications}

Hartung, K., Svensson, G., Struthers, H., Deppenmeier, A.-L., and Hazeleger, W.: An EC-Earth coupled atmosphere-ocean single-column model (AOSCM. v1_EC-Earth3) for studying coupled marine and polar processes, Geoscientific Model Development, 11, 41174137, 2018

Prodhomme, C., Voldoire, A., Exarchou, E., Deppenmeier, A.-L., García-Serrano, J., and Guemas, V.: How does the seasonal cycle control equatorial Atlantic interannual variability?, Geophysical Research Letters, 46(2), 916-922, 2019

Voldoire, A., Exarchou, E., Sanchez-Gomez, E., Demissie, T., Deppenmeier, A.-L., Frauen, C., Goubanova, K., Hazeleger, W., Keenlyside, N., Koseki, S., et al.: Role of wind stress in driving SST biases in the Tropical Atlantic, Climate Dynamics, pp. 1-24, 2019

Lancker, K., Deppenmeier, A.-L., Demissie, T., and Schmidt, J. O.: Climate change adaptation and the role of fuel subsidies: An empirical bio-economic modeling study for an artisanal open-access fishery, PloS one, 14.8: e0220433, 2019 


This research received funding from the European Union's Seventh Framework Programme for research, technological development and demonstration under grant agreement no 603521.

Financial support from Wageningen University for printing this thesis is gratefully acknowledged.

Cover design by Jessica Loriaux - www.mariemaakt.com Printed by proefschriften.nl 